\title{
Regulation of Bim in Health and Disease
}

\author{
Ronit Vogt Sionov ${ }^{1}$, Spiros A. Vlahopoulos ${ }^{2}$ and Zvi Granot ${ }^{1}$ \\ 1 Department of Developmental Biology and Cancer Research, Institute for Medical Research Israel Canada, Hebrew \\ University, Hadassah Medical School, Jerusalem, Israel \\ ${ }^{2}$ First Department of Pediatrics, University of Athens, Horemeio Research Laboratory, Thivon and Levadias, Goudi, Athens, \\ Greece
}

Correspondence to: Ronit Vogt Sionov, email: ronitsionov@gmail.com

Keywords: Bim, apoptosis, cancer, autoimmunity, neurodegenerative diseases

Received: July 28, $2015 \quad$ Accepted: August 08, 2015

Published: September 05, 2015

This is an open-access article distributed under the terms of the Creative Commons Attribution License, which permits unrestricted use, distribution, and reproduction in any medium, provided the original author and source are credited.

\section{ABSTRACT}

The BH3-only Bim protein is a major determinant for initiating the intrinsic apoptotic pathway under both physiological and pathophysiological conditions. Tight regulation of its expression and activity at the transcriptional, translational and post-translational levels together with the induction of alternatively spliced isoforms with different pro-apoptotic potential, ensure timely activation of Bim. Under physiological conditions, Bim is essential for shaping immune responses where its absence promotes autoimmunity, while too early Bim induction eliminates cytotoxic $\mathbf{T}$ cells prematurely, resulting in chronic inflammation and tumor progression. Enhanced Bim induction in neurons causes neurodegenerative disorders including Alzheimer's, Parkinson's and Huntington's diseases. Moreover, type I diabetes is promoted by genetically predisposed elevation of Bim in $\beta$-cells. On the contrary, cancer cells have developed mechanisms that suppress Bim expression necessary for tumor progression and metastasis. This review focuses on the intricate network regulating Bim activity and its involvement in physiological and pathophysiological processes.

\section{INTRODUCTION}

Understanding the dynamic nature of apoptotic regulators is very important in the design of intervention schemes for a number of debilitating conditions. It has become apparent that many pharmaceutical agents target only the initiating step in an intracellular signal cascade. While this is important, because an initiating step gets subsequently amplified, in many diseases a downstream step malfunctions, making most available drug treatments futile.

Over the past decade, Bim has emerged as an essential pro-apoptotic protein for initiating the intrinsic apoptotic pathway under many physiological and pathophysiological conditions. The complex network regulating its expression and activity has made it possible to manipulate cell death at several nodal points. A fine balance in the intracellular expression levels of Bim and its regulatory proteins is crucial for properly regulating apoptosis. This review will take us into a journey through the fascinating world of Bim.

\section{THE STRUCTURE OF BIM}

\subsection{General Aspects of Bim}

The BH3-only proteins participate in vital biological processes, and their absence contributes to autoimmunity and neoplasia [1, 2]. Bim is a Bcl-2 homology 3 (BH3)only protein that was discovered by O'Connor et al. [3] in 1998, while screening for proteins binding the antiapoptotic $\mathrm{Bcl}-2$ protein, giving raise to its name $B \mathrm{cl}-2$ interacting mediator of cell death. In the same year, Hsu et al. [4] discovered the same gene using Mcl-1 as a bait and termed the gene Bcl-2 related ovarian death agonist (BOD). Its official gene name is now Bcl-2-like 11 (Bcl2L11/apoptosis facilitator). The Bim gene is conserved in diverse mammalian species [4]. Bim function seems also to be conserved in non-mammalian vertebrates. A zebrafish ortholog of mammalian Bim was found to be the most toxic product of the zebrafish BH3-only genes examined, sharing this characteristic with the mammalian Bim gene [5].

Bim proteins are expressed in a wide variety 
of tissues including brain, heart, kidney, liver, lung, ovary, testis, spleen, thymus and trachea, but are most prominently expressed by cells of hematopoietic origin [6]. In the yeast two-hybrid protein-protein interaction assay, Bim interacts with the anti-apoptotic Bcl-2 proteins Mcl-1, Bcl-2, Bcl-xL, Bcl-w, Bfl-1 and Epstein-Barr virus (EBV) BHRF-1, but not with the pro-apoptotic Bcl-2 proteins Bad, Bak, Bok and Bax [4]. In mammalian cells, Bim can engage all anti-apoptotic proteins of the Bcl-2 superfamily, making it an efficient killer [1]. The BH3 domain becomes inserted into a hydrophobic groove of the pro-survival relatives [1]. The hydrophobic C-terminal part of Bim (MVILRLLRYIVRLVW) targets the protein to intracellular membranes [3]. In addition, Bim can directly interact with Bax and Bak, leading to mitochondrial outer membrane permeabilization [7-11]. Genetic analyses by Merino et al. [11] showed that only the Bim BH3 domain, but not other $\mathrm{BH} 3$ sequences inserted into the Bim protein, could rescue the leukocyte accumulation and autoimmune phenotype of Bim knockout (KO) mice, emphasizing the unique killing potency of Bim BH3. Thus, Bim may promote apoptosis by both acting as a death agonist and survival antagonist [8]. Other pro-apoptotic members of the BH3-only family include Bad, Bik, Bmf, Bid, Hrk, Noxa and Puma [12]. Bim often acts in concert with these pro-apoptotic proteins, which may explain why apoptosis is only partly compromised in Bim $\mathrm{KO}$ mice, as seen for glucocorticoid-induced apoptosis of thymocytes [2]. In contrast to normal cells, apoptosis of cancer cells often shows a complete dependence on Bim [13, 14]. The BH3-only pro-apoptotic proteins act upstream of the multi-domain pro-apoptotic proteins Bak, Bax and Bok, which have three $\mathrm{BH}$ domains $(\mathrm{BH} 1, \mathrm{BH} 2$ and $\mathrm{BH} 3)$. Double knockout of Bax and Bak often leads to complete resistance to apoptosis mediated by the intrinsic apoptotic pathway [15].

The multiple defects in Bcl-2/- mice can be prevented by loss of Bim, including the severe lymphopenia [16]. Even the loss of only one Bim allele was sufficient for the correction of the disorders, suggesting that the Bim levels set the threshold for initiation of apoptosis in several tissues [16].

\subsection{Bim Isoforms}

The gene locus of Bcl2L11 is located at chromosome 2 q13 in human. The gene has 6 exons, and undergoes alternative splicing to form at least 18 different isoforms transcribed from mRNA harboring 3-6 exons of which 2-4 are coding exons (Figure 1 and Supplementary Table 1). The three major alternative transcription variants of Bim described in mouse give raise to Bim $_{\mathrm{EL}}$ (Extra Long) (196

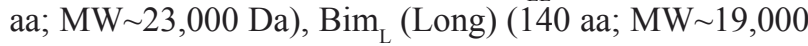
$\mathrm{Da})$ and $\mathrm{Bim}_{\mathrm{S}}$ (Short) (110 aa; MW 15,000 Da), where Bim $_{\mathrm{EL}}$ is the most abundant $[3,17]$. In human, these isoforms correspond to $\mathrm{Bim}_{\mathrm{EL}}$ (Bcl2L11 isoform 1) (198 aa; $22.1 \mathrm{kDa}), \operatorname{Bim}_{\mathrm{L}}(\mathrm{Bcl} 2 \mathrm{~L} 11$ isoform 6) (138 aa; 15.9
$\mathrm{kDa})$ and $\mathrm{Bim}_{\mathrm{S}}(\mathrm{Bcl} 2 \mathrm{~L} 11$ isoform 11) (108 aa; $12.7 \mathrm{kDa})$. $\operatorname{Bim}_{L}$ and $\operatorname{Bim}_{\mathrm{S}}$ are formed by alternative splicing within exon 2. Bim ${ }_{\mathrm{EL}}$ is the most abundant form in thymocytes and $\mathrm{T}$ cells, whereas $\mathrm{Bim}_{\mathrm{S}}$ is almost undetectable [18, 19]. All three isoforms are induced by glucocorticoids in thymocytes [20] and pre-B acute lymphoblastic leukemia [21].

All of the major isoforms contain a consensus BH3 domain of 9 amino acids (LRRIGDEFN) forming an amphipathic $\alpha$ helix, but lack other $\mathrm{BH}$ domains (BH1, $\mathrm{BH} 2$ and $\mathrm{BH} 4$ ) found in channel-forming Bcl-2 family proteins $[3,4]$. Bim $_{\mathrm{EL}}$ and $\mathrm{Bim}_{\mathrm{L}}$, but not $\mathrm{Bim}_{\mathrm{S}}$, possess the dynein light (L) chain-binding domain (DBD) encoded by exon $\mathrm{E} 2 \mathrm{C}$. Bim $\mathrm{EL}_{\mathrm{L}}$ and $\mathrm{Bim}_{\mathrm{L}}$ are released from microtubules in response to apoptotic stimuli, making them available for interaction with anti-apoptotic proteins such as Bcl-2 [22].

All 3 isoforms induce apoptosis; the shortest being the most potent [3]. The latter can be explained by the ability of $\mathrm{Bim}_{\mathrm{S}}$ to directly bind the pro-apoptotic Bax protein $[9,23,24]$, together with the absence of sequestration to the cytoskeleton [22] and posttranslational regulation $[17,25]$. There are some cellspecific effects. For instance, enforced expression of Bim $_{L}$

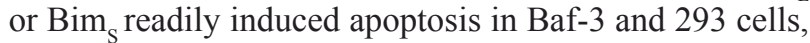
while Bim $_{\mathrm{S}}$, but not Bim ${ }_{\mathrm{L}}$, killed glioma cells [26, 27].

Upregulated expression of $\mathrm{Bim}_{\mathrm{S}}$ in epithelial cells was followed by its rapid mitochondrial translocation and mitochondrial insertion in the absence of any detectable binding to anti-apoptotic Bcl-2 proteins [24]. This caused mitochondrial recruitment and activation of Bax and apoptosis. The mitochondrial targeting, but not binding to Bcl-2 or Mcl-1, was required for apoptosis induction by $\mathrm{Bim}_{\mathrm{S}}$ [24]. In yeast, $\mathrm{Bim}_{\mathrm{S}}$ enhanced the killing activity of Bax in the absence of anti-apoptotic Bcl-2 proteins [24].

Additional isoforms have been described in human with differential pro-apoptotic activity [9, 28-30] (Figure 1 and Supplementary Table 1). U et al. [28] described six isoforms of human $\operatorname{Bim}(\operatorname{Bim} \alpha 1, \alpha 2$, and $\beta 1-4)$ that lacked the $\mathrm{C}$-terminal hydrophobic region. Among these isoforms, only $\alpha 1$ and $\alpha 2$ contained a $\mathrm{BH} 3$ domain and were pro-apoptotic, although less potent than the classical isoforms [28]. Marani et al. [9] described six isoforms that they termed BimAC, BimABC, BimAD, BimACD, BimA and BimABCD, all having a truncated $\mathrm{C}$-terminus, ending with either GIFE or LEK instead of the classical hydrophobic region. They showed that the small BimAD isoform that encodes for an 80 aa protein and harbors the BH3 domain, is pro-apoptotic [9]. Chen et al. [29] defined the BimAD as Bima3, and showed that this isoform is proapoptotic to a similar extent as Bima2, but is less potent than $\operatorname{Bim}_{\mathrm{S}}$ and $\mathrm{Bim}_{\mathrm{L}}$. Some of the isoforms described by Marani et al. [9] are similar to those described by U et al. [28] (Supplementary Table 1). Liu et al. [30] described an isoform termed Bim $\gamma$ (112 aa; MW 15,000), that is generated as a result of a retention of a 126-bp of intron 2 of the Bim gene. As the sequence of intron 2 contains a 
stop codon, the Bim $\gamma$ isoform contains only the E2A,E2B, $\mathrm{E} 2 \mathrm{C}$ coding exons and the intron 2 until reaching the premature TGA stop codon. It contains the dynein-binding domain (DKSTQT) presented in E2C, but lacks the classical BH3 domain encoded by exon 4 [30]. A BH3-like domain (LEDIGD instead of LRRIGD) is formed from the intron sequence that may be responsible for its proapoptotic and growth-inhibitory function [30].

\subsection{Bim Polymorphism}

$\mathrm{Ng}$ et al. [31] observed that a common intronic 2903 bp-deletion polymorphism within intron 2 in the Bim gene switched Bim splicing from exon 4 to exon 3 leading to the preferential expression of Bim $\gamma$. This deletion polymorphism occurs naturally in $12.9 \%$ of East-
Asian individuals and is observed in some individuals with chronic myeloid leukemia (CML) or epidermal growth factor receptor (EGFR)-mutated non-small-cell lung cancer (NSCLC) that conferred resistance to tyrosine kinase inhibitors such as gefitinib [31, 32]. Of note, the resistance could be overcome by the $\mathrm{BH} 3$-mimetic drug ABT-737 [31] or by combining gefitinib with the histone deacetylase inhibitor vorinostat that increased the splicing to exon 4, leading to augmented expression of the proapoptotic $\mathrm{BH} 3$-containing Bim [32].

The intronic deletion polymorphism may explain the heterogeneic response of cancer patients to tyrosine kinase inhibitors [31]. The progression free survival following EGFR tyrosine kinase inhibitor treatment of NSCLC was significantly shorter in patients with Bim polymorphism

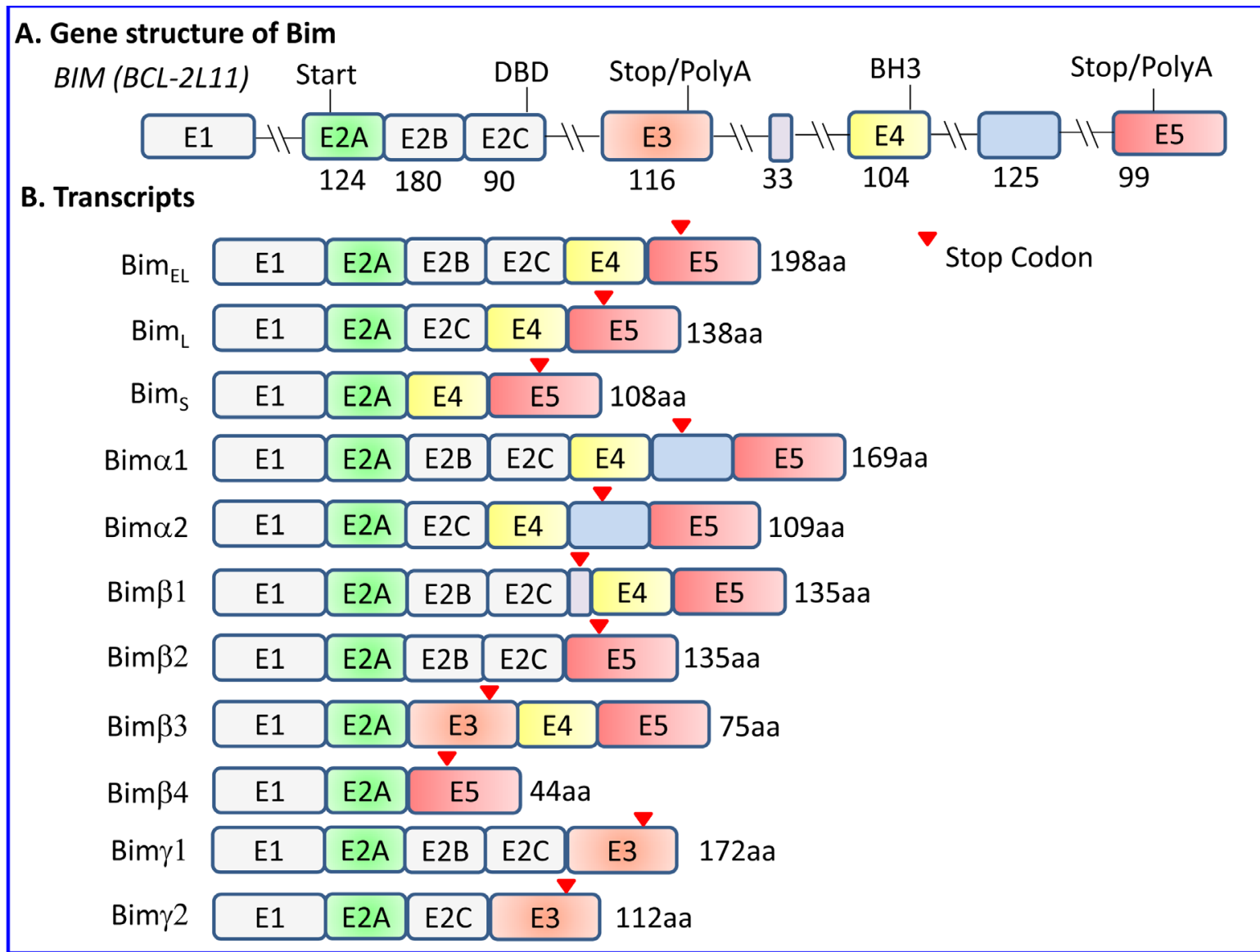

Figure 1: The gene structure of Bim and its major isoform transcripts. A. Presentation of the Bim (Bcl-2L11) gene structure according to the nomenclature of $\mathrm{U}$ et al. [28]. There has been much confusion in the literature concerning the nomenclature of the exon numbers where some research groups have denoted exons E2A, E2B and E2C as exons 2, 3 and 4 respectively, and exons E3, E4 and E5 as exons 5,6 and 7, respectively. As there is no intron between $\mathrm{E} 2 \mathrm{~A}$ and $\mathrm{E} 2 \mathrm{~B}$, and $\mathrm{E} 2 \mathrm{~B}$ and $\mathrm{E} 2 \mathrm{C}$, these regions are part of one exon, where intraexonal alternative splicing gives raise to the inclusion or exclusion of the E2B and/or E2C region. DBD - Dynein-binding domain. The BH3 domain is located in exon E4. The numbers beneath the exons refer to the amount of coding nucleotides in each exon. B. Presentation of various Bim isoform transcripts formed by alternative splicing. $\mathrm{Bim}_{\mathrm{EL}}, \mathrm{Bim}_{\mathrm{L}}$ and $\mathrm{Bim}_{\mathrm{S}}$ are the major classical isoforms, but also other isoforms have been identified as described in Section 1.2. Bima1-2 and Bim $\beta 1-4$ were described by U et al. [28], while Bim $\gamma$ by $\mathrm{Ng}$ et al. [31] that was coined Bim $\gamma 2$ by Anczukow et al. [229]. The latter research group characterized an additional Bim $\gamma$ isoform (Bim $\gamma 1$ ) that also retained the E2B exon [229]. Although $\mathrm{Ng}$ et al. [31] claim for a mutual exclusion of exon E3 and E4, the Bim $\beta 3$ isoform described by U et al. [28] does contain both exons. As the E3 exon contains a stop codon, its inclusion leads to a truncated protein lacking the pro-apoptotic BH3 domain. The Bim-ABCD, Bim-ACD and Bim-AD described by Marani et al. [9] corresponds to Bima1, Bima2 and Bima3. Bima3 resembles Bima2, but lacks E2C. 
(6.6 months) than those with wild-type Bim (11.9 months) [31]. However, others couldn't find an association of Bim deletion polymorphism and intrinsic resistance to tyrosine kinase inhibitors [33].

Another study showed that acute lymphoblastic leukemia patients harboring the single nucleotide BimC29201T (rs724710) polymorphism in exon 4 had shorter overall survival [34]. Overall survival was even shorter in patients with both Bim polymorphism and Mcl1 gene polymorphism (G486T) [34]. The single nucleotide polymorphism $\mathrm{C}>\mathrm{T}$ in Bim affected the inclusion of exon 3 and seems to contribute to drug resistance [34].

\subsection{Bim Knockout (KO) Mice}

A significant number of bim-null mice die in utero before E9.5, suggesting that Bim plays a role in development [2]. These mice accumulate lymphoid, myeloid and plasma cells and develop autoimmune kidney disease due to impaired apoptosis [2]. Bim-deficient mice have a higher number of B cells, CD4 and CD8 singlepositive $T$ cells, macrophages and granulocytes in the periphery. Expansion of the $\mathrm{B}$ cell population is associated with accumulation of serum immunoglobulins [2]. The abnormal increase in serum levels of IgM and IgG could be due to protection of plasma cells from endoplasmic reticulum (ER) stress-induced apoptosis, which in lymphoid and certain other cell types requires Bim [35]. The sensitivity of pre-B cells and autoreactive $B$ cells to apoptotic stimuli was low in $\mathrm{Bim}^{-/}$mice $[2,36]$. With age, Bim KO mice develop splenomegaly, lymphadenopathy, and hyper-gammaglobulinemia [2]. Although Bim is required for deletion of autoreactive thymocytes, Bimdeficient mice do not succumb to extensive organ-specific autoimmune disease, which may be due to an increase in T regulatory $\left(\mathrm{T}_{\text {reg }}\right)$ cells [37-40], impaired $\mathrm{T}$ cell activation [41] and reduced apoptotic sensitivity of the Bim-deficient target cells (See Section 4). Bim KO mice also showed gastric abnormality due to excessive accumulation of cells in the gastric epithelial layer [42].

In $\mathrm{T}$ cells, loss of Bim increases $\mathrm{T}$ cell production and function in interleukin-7 receptor (IL-7R; CD127)deficient mice [43]. Bim deficiency can partially rescue $\mathrm{B}$ cell development in mice deficient for the crucial $\mathrm{B}$ cell growth factor IL-7 [44]. Bim deficiency attenuates hematopoietic cell death in the fetal liver of Bcl-x-deficient mice, and could rescue testicular degeneration in $\mathrm{Bcl}-\mathrm{x}^{+/-}$ mice [45]. However, Bim deficiency couldn't prevent neuronal cell death in Bcl-x-deficient mice [45]. Loss of Bim renders lymphocytes refractory to paclitaxel (Taxol), ionomycin and cytokine deprivation, and partial resistance to glucocorticoids [2]. Death of thymocytes recognizing superantigens (Mtv-9 and Staphylococcus enterotoxin B) and male antigen HY was almost completely blocked in $\mathrm{Bim}^{-/}$mice [46]. Deletion of antigen-activated T cells during the shutdown of immune responses is also hindered in these mice [47].
Further studies show that Puma co-operates with Bim in apoptosis induction during lymphocyte development [48]. The absence of Puma or Bim renders thymocytes and mature lymphocytes refractory to varying degrees to death induced in vitro by growth factor withdrawal, DNA damage or glucocorticoids [49]. $\mathrm{Bim}^{-1 /}$ Puma $^{-/}$mice develop multiple postnatal defects that are not observed in the single knockout mice [48]. Hyperplasia of lymphatic organs is comparable with that observed in mice overexpressing Bcl-2 in all hematopoietic cells, exceeding the hyperplasia observed in $\mathrm{Bim}^{-/}$mice [48]. Mice deficient for both Puma and Bim spontaneously developed autoimmunity in multiple organs, and their T cells could transfer organ-specific autoimmunity [50]. Puma- and Bim-double-deficient mice showed accumulation of mature, single-positive thymocytes, suggesting that an additional defect in thymic deletion is the basis for the autoimmune disease [50]. Transgenic mouse models of thymocyte deletion by peripheral neoantigens confirmed that the loss of Bim and Puma allowed increased numbers of autoreactive thymocytes to escape deletion [50].

Deficiency of Bim, but not Puma, partially rescued $B$ cell development in the absence of IL-7 [51]. The numbers of both sIgM-negative and sIgM-positive B cells were markedly increased in the bone marrow of recipients lacking IL-7 upon reconstitution with Bim-deficient hematopoietic progenitors, compared with their control or Puma-deficient counterparts [51]. The augmentation of B cell lymphopoiesis in the absence of Bim was reflected in the mature peripheral compartment by an increase in both the number of immature and mature B cells in the spleen and in the circulating IgM levels [51].

Mice lacking both Bim and Bik showed similar hematopoietic alterations as Bim-deficient mice [52]. However, the double Bim/Bik KO male mice were infertile with reduced testicular cellularity and no spermatozoa [52]. The testis of young Bim/Bik double KO male mice had increased numbers of spermatogonia and spermatocytes, suggesting that spermatogenesis fails due to overwhelming amounts of supporting Sertoli cells [52].

\section{ROLE OF BIM IN APOPTOSIS}

\subsection{Indirect and Direct Apoptosis Induction by Bim}

Bim has been implicated in the regulation of intrinsic cell death induced by a large number of stimuli, including growth factor or cytokine deprivation, calcium flux, ligation of antigen receptors on $\mathrm{T}$ and $\mathrm{B}$ cells, loss of adhesion (anoikis), glucocorticoids, microtubule perturbation and tyrosine kinase inhibitors (Tables $1,2,3,4,5,6,7)$. It has been shown to be critical for apoptosis in $\mathrm{B}$ and $\mathrm{T}$ lymphocytes, macrophages and granulocytes [53]. The pioneer studies by O'Connor et al. [3] and Hsu et al. [4], showed that overexpression of Bim in Chinese hamster ovary (CHO) cells or 293T human embryonic 
Table 1: Bim Function in The Immune System.

\begin{tabular}{|c|c|c|}
\hline Apoptotic Stimulus & Remarks & References \\
\hline $\begin{array}{l}\text { Negative selection } \\
\text { of self-reactive } \\
\text { thymocytes }\end{array}$ & $\begin{array}{l}\text { - Thymocytes lacking Bim are refractory to apoptosis induced by TCR- } \\
\text { CD3 stimulation. } \\
\text { Bim is required for apoptosis of } \mathrm{CD}^{+} \mathrm{CD} 8^{+} \text {thymocytes induced by high- } \\
\text { affinity antigens. } \\
\text { - Bim is involved in clonal deletion of thymocytes recognizing tissue- } \\
\text { restricted antigens (TRAs), but not superantigen-mediated apoptosis. } \\
\text { - Autoreactive NODBim }{ }^{-/} \text {thymocytes receiving strong TCR signals that } \\
\text { would normally delete them, escape apoptosis and are diverted into } \mathrm{T}_{\text {reg }} \\
\text { cells. } \\
\text { Bim is essential for deletion of } \mathrm{CD} 4^{+} \mathrm{CD} 8^{-} \mathrm{CD} 24^{-} \text {thymocytes in response } \\
\text { to TCR ligation. }\end{array}$ & $\begin{array}{l}{[40,46,401,} \\
402,404- \\
406,416]\end{array}$ \\
\hline $\begin{array}{l}\text { Activated } \mathrm{T} \text { cell } \\
\text { death }\end{array}$ & $\begin{array}{l}\text { - Bim is a key regulator of } \mathrm{T} \text { cell apoptosis during the contraction phase of } \\
\mathrm{CD} 8^{+} \mathrm{T} \text { cell response. } \\
\text { - } \mathrm{V} \beta 8^{+} \mathrm{T} \text { cells from Bim-deficient mice are resistant to staphylococcus } \\
\text { enterotoxin B-induced } \mathrm{T} \text { cell death. } \\
\text { - While the Bim levels did not change after exposure to staphylococcal } \\
\text { enterotoxin } \mathrm{B} \text {, the Bcl-2 levels decreased. } \\
\text { - Shutdown of an acute } \mathrm{T} \text { cell response to herpes simplex virus involved } \\
\text { Bim. } \\
\text { Bim deficiency increases antigen-specific } \mathrm{CD}^{+} \mathrm{T} \text { cell responses during } \\
\text { viral infection. }\end{array}$ & $\begin{array}{l}{[47,395,} \\
398,399]\end{array}$ \\
\hline $\begin{array}{l}\text { B7-H1 (PD-L1)- } \\
\text { induced apoptosis of } \\
\text { effector T cells }\end{array}$ & $\begin{array}{l}\text { - B7-H1 (PD-L1) engagement with its receptor PD-1 promotes apoptosis of } \\
\text { effector T cells through upregulation of Bim. } \\
\text { - More memory } \mathrm{CD}^{+} \mathrm{T} \text { cells were generated in B7-H1-deficient mice } \\
\text { following immunization. }\end{array}$ & [409] \\
\hline $\begin{array}{l}\text { Elimination of } \\
\text { poorly functional } \\
\text { Th1 responder cells }\end{array}$ & 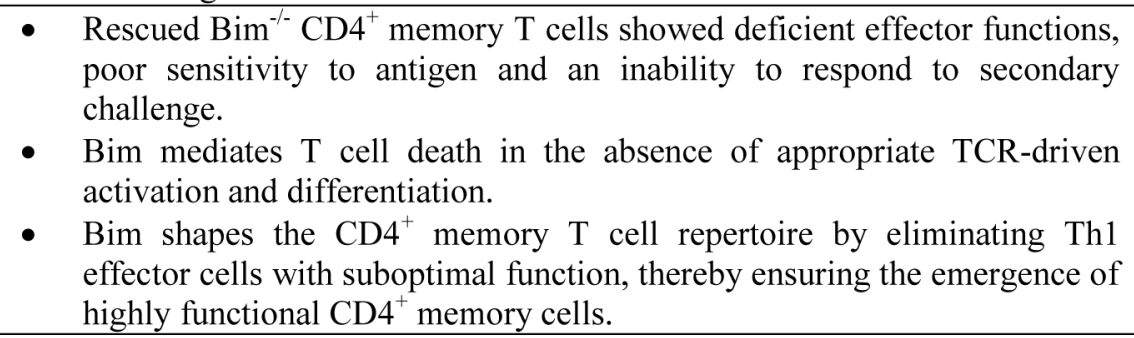 & [408] \\
\hline $\begin{array}{l}\text { Regulation of T } \\
\text { memory cells }\end{array}$ & $\begin{array}{l}\text { - The absence of Bim increased the effector } \mathrm{CD}^{+} \mathrm{T} \text { cell population with } \\
\text { more memory potential. } \\
\text { - Survival of memory } \mathrm{T} \text { cells depends on TRAF1-mediated Bim } \\
\text { downregulation. } \\
\text { The absence of Bim-mediated death of lymphocytic choriomeningitis } \\
\text { virus-specific } \mathrm{CD} 4^{+} \text {and } \mathrm{CD} 8^{+} \mathrm{T} \text { cells in vivo leads to increased } \\
\text { differentiation, even of } \mathrm{CD} 127^{10} \mathrm{~T} \text { cells, into memory } \mathrm{T} \text { cells. }\end{array}$ & $\begin{array}{l}{[411,422,} \\
471]\end{array}$ \\
\hline $\begin{array}{l}\text { Regulation of T } \\
\text { regulatory cells }\end{array}$ & $\begin{array}{l}\text { - In the absence of Bim, T regulatory cells accumulate rapidly, accounting } \\
\text { for }>25 \% \text { of the } \mathrm{CD}^{+} \mathrm{T} \text { cells in aged mice. } \\
\text { - Rapid peripheral T regulatory cell turnover depends on Bim. } \\
\text { - Induced regulatory } \mathrm{T} \text { cells show decreased } \mathrm{Bcl}-2 \text { expression and increased } \\
\text { Bim expression and were more prone to apoptosis. } \\
\text { - } \mathrm{Rag}^{-/-} \text {hosts repopulated with Bim }{ }^{-/-} \text {conventional } \mathrm{CD} 4^{+} \mathrm{T} \text { cells resulted in } \\
\text { a larger induction of regulatory } \mathrm{T} \text { cells than mice given wild-type } \\
\text { conventional } \mathrm{CD} 4^{+} \text {cells. } \\
\text { Bim deficient mice showed increased numbers of } \mathrm{CD} 25^{\text {low }} \mathrm{Foxp} 3^{+} \text {cells in } \\
\text { the thymus and peripheral lymph tissue. The } \mathrm{CD} 25^{\text {low }} \mathrm{Foxp}^{+} \mathrm{CD}^{+} \text {cells } \\
\text { were anergic and had weaker regulatory function than } \mathrm{CD} 25^{\text {high }} \mathrm{Foxp} 3^{+}\end{array}$ & $\begin{array}{l}{[37-39,394,} \\
413]\end{array}$ \\
\hline
\end{tabular}




\begin{tabular}{|c|c|c|}
\hline & $\mathrm{CD}^{+} \mathrm{T}$ cells from the same mice. & \\
\hline $\begin{array}{l}\text { B cell antigen } \\
\text { receptor (BCR) } \\
\text { stimulation-induced } \\
\text { apoptosis }\end{array}$ & $\begin{array}{l}\text { - B lymphocytes lacking Bim are refractory to apoptosis induced by BCR } \\
\text { ligation. } \\
\text { - The loss of Bim also inhibited deletion of autoreactive B cells in vivo in a } \\
\text { B cell tolerance model. } \\
\text { - Siglecs induce tolerance to cell surface antigens by Bim-dependent } \\
\text { deletion of antigen-reactive B cells. } \\
\text { - Cross-linked anti- } \mu \text { antibodies that trigger apoptosis of human B } \\
\text { lymphocytes, induce ERK-dependent downregulation of Bim } \text { EL }_{\text {L }} \text { with } \\
\text { simultaneous upregulation of the Bim } \text { and Bim }_{\mathrm{S}} \text { isoforms. }\end{array}$ & {$[36,83,429]$} \\
\hline $\begin{array}{l}\text { Elimination of } \\
\text { autoreactive B cells }\end{array}$ & - Bim is involved in the elimination of autoreactive and anergic B cells. & {$[36,426]$} \\
\hline $\begin{array}{l}\text { Superantigen- } \\
\text { mediated B cell } \\
\text { death }\end{array}$ & $\begin{array}{l}\text { - The microbial virulence factor protein A of Staphylococcus aureus } \\
\text { interact with evolutionarily conserved BCR-binding sites to induce a form } \\
\text { of Bim-dependent activation-associated B cell death. }\end{array}$ & [427] \\
\hline $\begin{array}{l}\text { Apoptosis of low- } \\
\text { affinity-expressing } \\
\text { B cells. }\end{array}$ & $\begin{array}{l}\text { - After immunization, Bim-deficient mice showed persistence of memory B } \\
\text { cells lacking affinity-enhancing mutations in their immunoglobulin genes } \\
\text { and antibody-forming cells secreting low-affinity antibodies. }\end{array}$ & [392] \\
\hline $\begin{array}{l}\text { Spontaneous and } \\
\text { stress-induced } \\
\text { apoptosis of } \\
\text { granulocytes }\end{array}$ & $\begin{array}{l}\text { - Bim deficiency renders granulocytes resistant to cytokine withdrawal and } \\
\text { cytotoxic drugs such as etoposide and paclitaxel. } \\
\text { GM-CSF treatment temporarily blocks apoptosis by inducing Mcl-1 with } \\
\text { rapid turnover and Bim, which limits GM-CSF-mediated prolonged } \\
\text { survival of neutrophils. }\end{array}$ & $\begin{array}{l}{[357,433,} \\
434]\end{array}$ \\
\hline $\begin{array}{l}\text { Phagocytosis- } \\
\text { induced apoptosis of } \\
\text { macrophages }\end{array}$ & $\begin{array}{l}\text { - Phagocytosis and intracellular killing of bacteria lead to apoptosis of } \\
\text { macrophages that involve TLR-, p38-and JNK-dependent upregulation of } \\
\text { Bim. } \\
\text { Phagocytosis-induced apoptosis was strongly reduced in } \mathrm{Bim}^{-/-} \\
\text {macrophages. }\end{array}$ & [430] \\
\hline $\begin{array}{l}\text { Spontaneous cell } \\
\text { death of dendritic } \\
\text { cells }\end{array}$ & $\begin{array}{l}\text { - Bim-deficient dendritic cells showed decreased spontaneous cell death } \\
\text { and induced more robust } \mathrm{T} \text { cell activation. }\end{array}$ & [432] \\
\hline $\begin{array}{l}\text { Antigen-specific } \\
\text { NK cell contraction }\end{array}$ & $\begin{array}{l}\text { - Antigen-specific NK cell contraction after mouse cytomegalovirus } \\
\text { infection depends on Bim. }\end{array}$ & {$[436]$} \\
\hline Mast cell apoptosis & $\begin{array}{l}\text { - Bim is induced together with Bcl-xL upon IgE receptor activation of mast } \\
\text { cells. }\end{array}$ & {$[358]$} \\
\hline Osteoclast apoptosis & $\begin{array}{l}\text { - Liver X receptor activation leads to osteoclast apoptosis through Bim } \\
\text { upregulation. }\end{array}$ & [645] \\
\hline $\begin{array}{l}\text { Cytokine } \\
\text { deprivation }\end{array}$ & $\begin{array}{l}\text { - Cytokine withdrawal leads to activation of FKHR-L1 in lymphocytes, } \\
\text { which is responsible for the upregulation of Bim expression. } \\
\text { - Early hematopoietic progenitor cells ( } \mathrm{Sca}^{+} \mathrm{I}^{+}, \mathrm{c}-\mathrm{Kit}^{+}, \mathrm{Lin}^{-} \text {) undergo rapid } \\
\text { apoptosis in the absence of cytokines concomitant with Bim induction. } \\
\text { - IL-3 signaling leads to phosphorylation of } \mathrm{Bim}_{\mathrm{EL}} \text { and its consequent } \\
\text { degradation in hematopoietic stem cells. } \\
\text { - IL-3 downregulates Bim through the Ras/MAPK and PI3K/Akt pathways. } \\
\text { - M-CSF deprivation of osteoclasts leads to Bim-dependent apoptosis. } \\
\text { - Bim deficiency prevented cytokine withdrawal-induced mast cell } \\
\text { apoptosis. } \\
\text { - } \mathrm{PGE}_{2} \text { increases mast cell death during cytokine deprivation by } \\
\text { augmenting Bim expression. }\end{array}$ & $\begin{array}{l}{[26,122} \\
358,360 \\
438,646]\end{array}$ \\
\hline $\begin{array}{l}\text { IL-21-induced } \\
\text { apoptosis of CLL }\end{array}$ & $\begin{array}{l}\text { - IL-21 induces apoptosis of chronic lymphoblastic leukemia (CLL) by } \\
\text { activating the STAT-1 pathway and Bim induction. } \\
\text { - IL-21 increased the cytotoxic effect of fludarabine and rituximab on CLL. }\end{array}$ & [190] \\
\hline
\end{tabular}


Table 2: Bim Function in The Nerve System.

\begin{tabular}{|c|c|c|}
\hline $\begin{array}{l}\text { Apoptotic } \\
\text { Stimulus }\end{array}$ & Remarks & References \\
\hline $\begin{array}{l}\text { Sympathetic } \\
\text { neuronal death } \\
\text { induced by NGF } \\
\text { deprivation }\end{array}$ & $\begin{array}{l}\text { - Nerve growth factor (NGF) withdrawal induced Bim } \mathrm{EL}_{\mathrm{E}} \text { expression and } \\
\text { apoptosis by a mechanism dependent on c-Jun. } \\
\text { - } \text { Bim deletion protected against neuronal apoptosis. } \\
\text { - NGF promoted MEK/MAPK-mediated phosphorylation of Bim } \mathrm{EL}_{\mathrm{EL}} \text { at } \\
\text { Ser109 and Thr110, thereby suppressing its activity. }\end{array}$ & $\begin{array}{l}{[125,311,} \\
355]\end{array}$ \\
\hline $\begin{array}{l}\beta \text {-Amyloid- } \\
\text { induced neuronal } \\
\text { apoptosis }\end{array}$ & $\begin{array}{l}\text { - Cdk4 and its downstream effector B-myb are required for } \beta \text {-amyloid- } \\
\text { dependent Bim induction and death in cultured neurons. } \\
\text { - } \beta \text {-Amyloid activates Mst-1-mediated nuclear translocation of FoxO3 that } \\
\text { is important for Bim imduction. }\end{array}$ & {$[352,388]$} \\
\hline $\begin{array}{l}\text { Thrombin- } \\
\text { induced apoptosis } \\
\text { of cultured } \\
\text { cerebral cortical } \\
\text { neurons }\end{array}$ & $\begin{array}{l}\text { - Cyclin D1, Cdk4 and Bim were shown to be involved in thrombin- } \\
\text { induced apoptosis of cultured cerebral cortical neurons. }\end{array}$ & [383] \\
\hline $\begin{array}{l}\text { Ischemic neuronal } \\
\text { cell death }\end{array}$ & $\begin{array}{l}\text { - Ischemia leads to neuronal cell death mediated by Notch- and NFKB- } \\
\text { dependent Bim upregulation. } \\
\text { - Bim-deficient mice showed decreased parenchymal loss in the } \\
\text { hippocampal area following neonatal hypoxia-ischemia. }\end{array}$ & {$[351,447]$} \\
\hline $\begin{array}{l}\text { Parkinson's } \\
\text { disease }\end{array}$ & $\begin{array}{l}\text { - Dysfunction of mitochondrial complex I leads to degeneration of } \\
\text { dopaminergic neurons through JNK-dependent activation of Bim. }\end{array}$ & [445] \\
\hline $\begin{array}{l}\text { Huntington's } \\
\text { disease }\end{array}$ & $\begin{array}{l}\text { - Overexpression of mutant Huntingtin protein leads to increased } \text { Bim }_{\mathrm{EL}} \\
\text { expression, and knockdown of Bim prevents apoptosis mediated by } \\
\text { mutant Huntingtin. }\end{array}$ & {$[180]$} \\
\hline $\begin{array}{l}\text { p75 Neurotrophin } \\
\text { receptor } \\
\text { (p75NTR) }\end{array}$ & $\begin{array}{l}\text { - Overexpression of p75NTR induced JNK-dependent phosphorylation of } \\
\text { Bim } \text { EL }_{\mathrm{L}} \text { at Ser65 in primary cerebellar granule neurons, which resulted in } \\
\text { apoptosis. }\end{array}$ & [324] \\
\hline
\end{tabular}

kidney cells led to apoptosis that could be prevented by the baculoviral caspase inhibitor P35, and the antiapoptotic Bcl-2, Bcl-xL and Bcl-w proteins. However, the more distant viral homologues adenovirus E1B19K and Epstein-Barr virus BHRF-1 were unable to prevent the pro-apoptotic effect of Bim [3]. The BH3 domain is essential for its pro-apoptotic function [3, 4]. A mutant Bim protein lacking the $\mathrm{BH} 3$ domain did not interact with Bcl-2, Bcl-xL or Bcl-w [3]. Although all three Bim isoforms in mouse bind $\mathrm{Bcl}-2, \mathrm{Bim}_{\mathrm{S}}$ antagonizes $\mathrm{Bcl}-2$ more effectively than $\mathrm{Bim}_{\mathrm{L}}$, while $\mathrm{Bim}_{\mathrm{EL}}$ was the least potent [3]. Besides neutralizing the anti-apoptotic proteins, Bim promotes apoptosis by binding to Bax leading to a conformation change in Bax that leads to its activation $[8$, 9] (Figure 2).

The pro-apoptotic effect of Bim depends on Bak and Bax [54], as do most apoptotic stimuli [15, 55]. A constitutively active form of Bim induces apoptosis in cells derived from either $\mathrm{Bax}^{-/}$or $\mathrm{Bak}^{-/}$animals, but failed to do so in $\mathrm{Bax}^{--} \mathrm{Bak}^{-/}$double $\mathrm{KO}$ cells [54]. Activation of Bak and Bax leads to homo-oligomerization and assembly within the mitochondrial outer membrane (MOM) followed by MOM permeabilization (MOMP), cytochrome $\mathrm{C}$ release, and initiation of the intrinsic apoptotic pathway $[12,56]$. Bim also leads to uncoupling of mitochondrial respiration and the subsequent increase in the cellular levels of reactive oxygen species (ROS) [57].

While many studies have demonstrated the ability of Bim to bind to anti-apoptotic proteins, less is known on how Bim activates Bax and Bak. In the indirect activation model, binding of $\mathrm{BH}$-only proteins with the anti-apoptotic Bcl-2 proteins leads to the release of Bak and Bax, making them available to induce apoptosis [58]. This displacement mechanism takes place during Tumor necrosis factor $\alpha(\mathrm{TNF} \alpha)$-induced apoptosis of $\mathrm{PC} 12$ and MCF7 cells [59]. This study showed that the interaction between Bcl-xL and Bax decreased after TNF $\alpha$ treatment, while the interaction between Bcl-xL and $\mathrm{Bim}_{\mathrm{L}}$ increased [59]. Similarly, UV-induced apoptosis led to Bim ${ }_{L}$ binding to Bcl-xL with subsequent Bax release [60]. TCR ligation of developing thymocytes led to upregulation of Bim, and increased Bim binding to Bcl-xL [46], while B cell antigen receptor (BCR) ligation led to increased Bim binding to $\mathrm{Bcl}-2$ [36]. However, the indirect Bax/Bak activation model seems to be only part of the story in light of the fact that only a small fraction of Bax and Bak is bound to pro-survival proteins, and in most cell types, Bax is located in the cytosol away from the anti-apoptotic proteins $[61,62]$. Moreover, Bim activation can also lead 
to apoptosis in the absence of anti-apoptotic proteins, e.g., following glucocorticoid treatment of double negative thymic lymphoma cells lacking anti-apoptotic Bcl-2 proteins [63-65]. Yamaguchi and Wang [66] used mutant $\mathrm{Bim}_{\mathrm{EL}}$ proteins to demonstrate that $\mathrm{Bim}_{\mathrm{EL}}$ may activate Bax directly, in addition to its binding to Bcl-2/ Bcl-xL. Also other studies showed that Bim, especially Bim , can directly activate $\operatorname{Bax}$ and $\operatorname{Bak}[9,23,24,67,68]$.

In the absence of apoptotic stimuli, the $\alpha 1$ helix of Bax keeps the $\alpha 9$ helix engaged in the dimerization pocket formed by the BH1-3 domains, maintaining Bax as a monomer in cytosol $[69,70]$. The C-terminal $\alpha 9$ helix of Bak is constitutively inserted in the mitochondrial outer membrane, but its activity is inhibited by VDAC2, which occupies the dimerization pocket of Bak to restrict Bak in the monomeric inactive conformation [71, 72]. Upon interaction of the $\mathrm{BH} 3$ helix of Bim with the $\alpha 1$ and $\alpha 6$ helices of Bax, the unstructured loop between $\alpha$ helices 1 and 2 is displaced, and the C-terminal helix 9 is mobilized for membrane translocation [10, 69, 73]. This leads to the exposure of the $\mathrm{Bax} \mathrm{BH} 3$ domain that propagates the death signal through an auto-activating interaction with the trigger site of inactive Bax monomers [73]. Activator $\mathrm{BH} 3$-only proteins remain associated with the N-terminally exposed Bax through the $\mathrm{BH} 1$ domain to drive homo-oligomerization [69].

With respect to Bak, its activation depends on the exposure of its $\mathrm{BH} 3$ domain, which becomes reburied in dimers [74]. The oligomerization involves the insertion of the $\mathrm{BH} 3$ domain of one Bak molecule into the groove of another [74]. The resulting BH3:groove dimers can be converted to larger oligomers that permeabilize mitochondria by an interface between $\alpha 6$ helices [75]. Genetic deletions of Bid, Bim and Puma prevented the

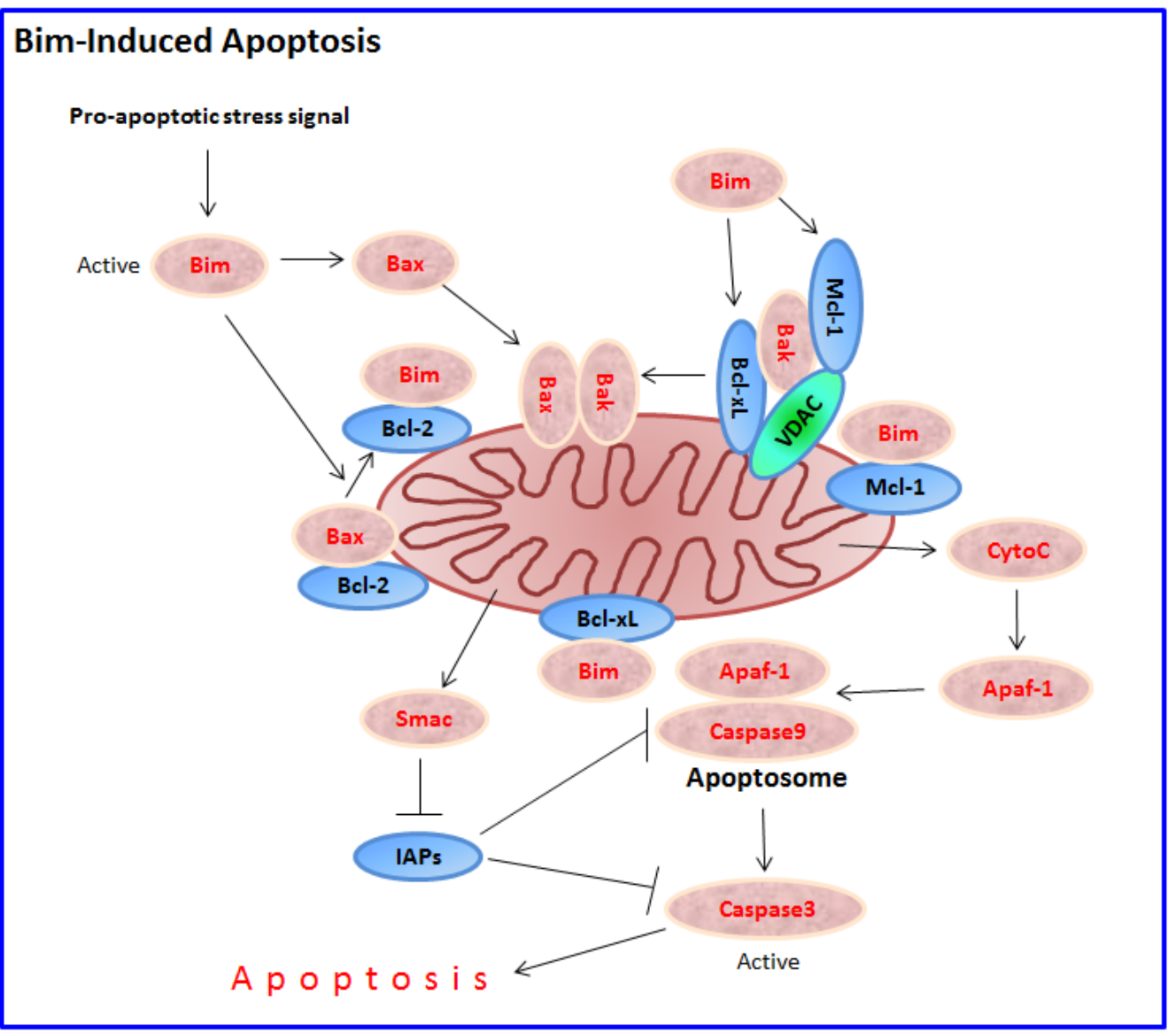

Figure 2: Bim-induced apoptosis. Following exposure to a pro-apoptotic stimulus, a sudden intracellular rise in free activated Bim molecules (e.g., by increased transcription and/or translation, increased alternative splicing in favor of Bim ${ }_{\mathrm{S}}$, and/or release of Bim from sequestered intracellular storages as a result of phosphorylation) initiates the intrinsic mitochondrial apoptotic pathway. Bim induces apoptosis by directly activating Bax and Bak, or indirectly by interacting with the anti-apoptotic proteins Bcl-2, Bcl-xL and Mcl-1, leading to the release and mitochondrial transfer of Bax and Bak. Under normal conditions, Bak is hold in check by Mcl-1, VDAC2 and Bcl-xL. $\mathrm{Bax} / \mathrm{Bak}$ oligomerization in the mitochondrial outer membrane results in dissipation of the mitochondrial outer membrane potential $\left(\Delta \psi_{\mathrm{m}}\right)$ and release of the apoptogenic proteins cytochrome $\mathrm{C}(\mathrm{CytoC}), \mathrm{Smac} / \mathrm{DIABLO}$ and HtrA2 into the cytosol. Cytochrome $\mathrm{C}$ activates Apoptotic protease activating factor 1 (Apaf-1) that facilitates the formation of the apoptosome where caspase 9 is activated to initiate the apoptotic cascade concluded with the activation of caspase 3. Smac/DIABLO antagonizes the anti-apoptotic function of Inhibitors of apoptosis protein (IAPs) such as XIAP, cIAP1 and cIAP2, thereby enhancing apoptosis induction by cytochrome C. 
homo-oligomerization of Bax and Bak, and cytochrome C-mediated activation of caspases in response to diverse death signals in neurons and $\mathrm{T}$ cells, suggesting that these three $\mathrm{BH} 3$-only proteins co-operate in activating Bax and Bak [68]. A recent study [76] showed that Bim preferentially activates Bax, while Bid preferentially activates Bak. This study contrasts previous studies showing direct binding of Bid BH3 helix with Bax [77] and Bim with Bak [78]. Puma was recently shown to directly bind and activate Bak and Bax [79-81], besides binding to Bcl-xL [81]. Weber et al. [78] showed that ABT-737-mediated release of Bak from Bcl-xL couldn't induce apoptosis unless Bim associates with oligomeric Bak to promote its conversion to a membrane-inserted pore.

While Bcl-2 is a biomarker of resistance to both chemotherapy and radiotherapy, the association of Bim with the anti-apoptotic Bcl-2 protein may explain why Bcl-2 overexpressing lung and breast cancer cells are more sensitive to microtubule-targeting agents such as paclitaxel and vinorelbine [82]. This paradox of an anti-apoptotic protein can be explained by the upregulation of Bim in lung cancer upon Bcl-2 overexpression that makes Bim easily available upon exposure to stress stimuli [82].

\subsection{Interaction of Bim with the Cytoskeleton}

In some cell types (e.g., breast carcinoma), Bim seems to affect cytoskeletal integrity through sequestration to microtubule-associated dynein motor complexes by binding tightly to the LC8 cytoplasmic dynein L (light) chain (DYNLL1) [22]. In hematopoietic cells, Bim is not associated with microtubules, but rather associates with Bcl-2-like proteins on mitochondria $[7,18,83]$. In B and multiple myeloma cells, Bim appears to be constitutively associated with Mcl-1. At induction of apoptosis, Bim is released from $\mathrm{Mcl}-1$, thus activating its pro-apoptotic function [84-86].

Bim $_{E L}$ and Bim $_{L}$, but not Bim $_{S}$ harbors the dyneinbinding domain (DKSTQT) $[22,30]$. Interaction of Bim with DYNLL1 facilitates the interaction of DYNLL1 with Beclin-1, leading to inhibition of autophagy [87]. Overexpression of Bim suppressed autophagy, whereas Bim KO cells showed enhanced autophagocytosis [87]. Under starvation conditions, Bim is phosphorylated by MAPK8/JNK, leading to the dissociation of the BimBeclin-1-DYNLL1 complex, which leads to activation of autophagy by Beclin-1 and initiation of apoptosis by Bim [88] (Figure 3). Especially the isoforms $\mathrm{Bim}_{\mathrm{EL}}$ and $\mathrm{Bim}_{\mathrm{L}}$ can interact with Beclin-1 [88]. Bim ${ }_{L}$ may also promote acidification of lysosomes required for the formation of autophagic vesicles [89].

UV-irradiation leads to phosphorylation of $\mathrm{Bim}_{\mathrm{L}}$ at Thr56 by activated JNK and consequent release from the microtubule, followed by translocation to the mitochondria [90]. Bim is also released from microtubule following paclitaxel (Taxol) treatment that interferes with the normal breakdown of microtubules during cell division [91]. The p53- and BRCA1-regulated stress protein GADD45a that has been implicated in the maintenance of genomic integrity, causes dissociation of Bim from microtubuleassociated components leading to Bim translocation to mitochondria [92]. The Bim accumulation in mitochondria enhances the interaction of Bim with Bcl-2, relieves Bax from Bcl-2-bound complexes, and subsequently results in release of cytochrome $\mathrm{C}$ into the cytoplasm [92]. Thus, Bim can be indirectly upregulated by the p53 tumor suppressor gene. Suppression of Bim inhibited GADD45a-induced apoptosis [92]. GADD45a interacts with and inhibits the function of elongation factor $1 \alpha(\mathrm{EF}-$ $1 \alpha$ ), a microtubule-severing protein that plays a role in maintaining cytoskeletal stability, leading to disruption of microtubule bundling [92]. Overexpression of EF-1 $\alpha$ leads to resistance to apoptosis induced by growth factor withdrawal, ER and genotoxic stress stimuli [93, 94].

\subsection{The Reciprocal Roles of Mcl-1 and Bim in Regulating Apoptosis}

Han et al. [95] described an intriguing interplay between Mcl-1 and Bim in Tumor necrosis factor-related apoptosis-inducing ligand (TRAIL)-induced apoptosis. In the absence of pro-apoptotic stimuli, Bim is sequestrated to $\mathrm{Mcl}-1$ in tumor cells. Upon stimulation with TRAIL, caspase 8 is activated and promotes degradation of Mcl-1, resulting in the release of Bim that triggers Bax-dependent apoptosis. The Mcl-1 expression level at the mitochondrial outer membrane determines the release efficiency for the apoptogenic proteins cytochrome $\mathrm{C}$, Second mitochondriaderived activator of caspase (Smac), and High temperature requirement serine protease A2 (HtrA2) in response to Bim [95]. Earlier studies by Herrant et al. [96] showed that Mcl-1 is cleaved by caspases during the induction of apoptosis in various cancer cells. The Mcl-1 cleavage results in the loss of the $\mathrm{BH} 4$ homolgy domain required for its anti-apoptotic activity [96]. Similarly, Mcl-1 could be degraded by the $\mathrm{T}$ cell granule serine protease granzyme $\mathrm{B}$, again releasing Bim that promotes apoptosis [97].

Mcl-1 is subjected to tight regulation at the level of protein stability. The degradation of Mcl-1 is often, but not always, required for initiation of apoptosis [64, 98-100]. The mRNA level of Mcl-1 decreases in response to various apoptotic stimuli such as UV irradiation and staurosporine [98]. Mcl-1 is a short-lived protein, positively regulated by the mTOR signaling pathway, while negatively by the E3 ligases Mcl-1 ubiquitin ligase E3 (Mule), F-Box and WD repeat domain containing 7 (Fbw7), Tripartite motif containing 17 (Trim17) and $\beta$-transducin repeat containing E3 ubiquitin protein ligase 1 ( $\beta$-TrCP1), that promote its degradation [101-106]. Phosphorylation of Mcl-1 by Glycogen synthase kinase 3 (GSK3) triggers the interaction of Mcl-1 with Fbw7 [106]. The microtubuletargeting agents Paclitaxel and Vinblastine induce phosphorylation-dependent, Fbw7-mediated degradation 
of Mcl-1 [107]. The mTOR complex 2 (mTORC2) stabilizes Mcl-1 by suppressing the GSK3-dependent and Fbw7-mediated degradation [108]. It is noteworthy that GSK3 is activated upon glucocorticoid-induced apoptosis, leading to interaction of GSK3 with Bim and induction of Bim-dependent apoptosis of lymphoma cells [63]. Thus, GSK3 has a dual function, the first is to reduce Mcl1 levels, and the other to enhance Bim activity, thereby fortifying apoptosis.

The VTLISFG in the BH1 domain of Mcl-1 seems to be important for regulating its degradation [109]. The deubiquitinase Ubiquitin-Specific Protease 9X (USP9X) stabilizes Mcl-1, thereby promotes cell survival [110]. Increased USP9X expression correlates with increased Mcl-1 protein in human follicular lymphomas and diffuse large B cell lymphomas [110]. Moreover, patients with multiple myeloma overexpressing USP9X have a poor prognosis [110]. Importantly, targeting USP9X attenuates B cell acute lymphoblastic leukemia cell survival and overcomes glucocorticoid resistance [111], a death process dependent on Bim [14].

A competing BH3-ligand derived from Bim interacted with Mcl-1 and prevented its interaction with Mule, leading to increased Mcl-1 expression [112]. This suggests that Bim needs to be released from Mcl-1 prior to its degradation by Mule [112]. However, binding of the p53-regulated pro-apoptotic Noxa protein to Mcl-1 leads to its degradation [113]. The Noxa-mediated degradation of Mcl-1 required Mule, but is also mediated through interruption of the deubiquitinase USP9X with Mcl-1 [113]. Also, Beclin-1 leads to destabilization of Mcl-1 through competitive binding to USP9X [114]. USP9X inhibition using WP1130 led to Mcl-1 inhibition and sensitization of solid tumors to various chemotherapeutic agents [115].

\section{REGULATION OF BIM EXPRESSION AND ACTIVITY}

The expression and pro-apoptotic activity of Bim are tightly regulated at several levels (Figures 4-7). Its transcription is regulated by several transcription factors that can either induce its expression (e.g., FoxO3a, E2F1, c-Myc, NF-Y, Smad1/3, Runx1-3, c-Jun and RelA) (Figure 4) or repress it (e.g., YY1, HoxB8, SPi-1/PU.1, PINCH-1, Pokemon) (Figure 5). The bim promoter is also epigenetically regulated through methylation of CpG dinucleotides at the 5' end of the Bim gene. The Bim transcript undergoes alternative splicing leading to translation of various Bim isoforms with differential pro-

\section{The Dual Role of Bim in Apoptosis and Autophagy}

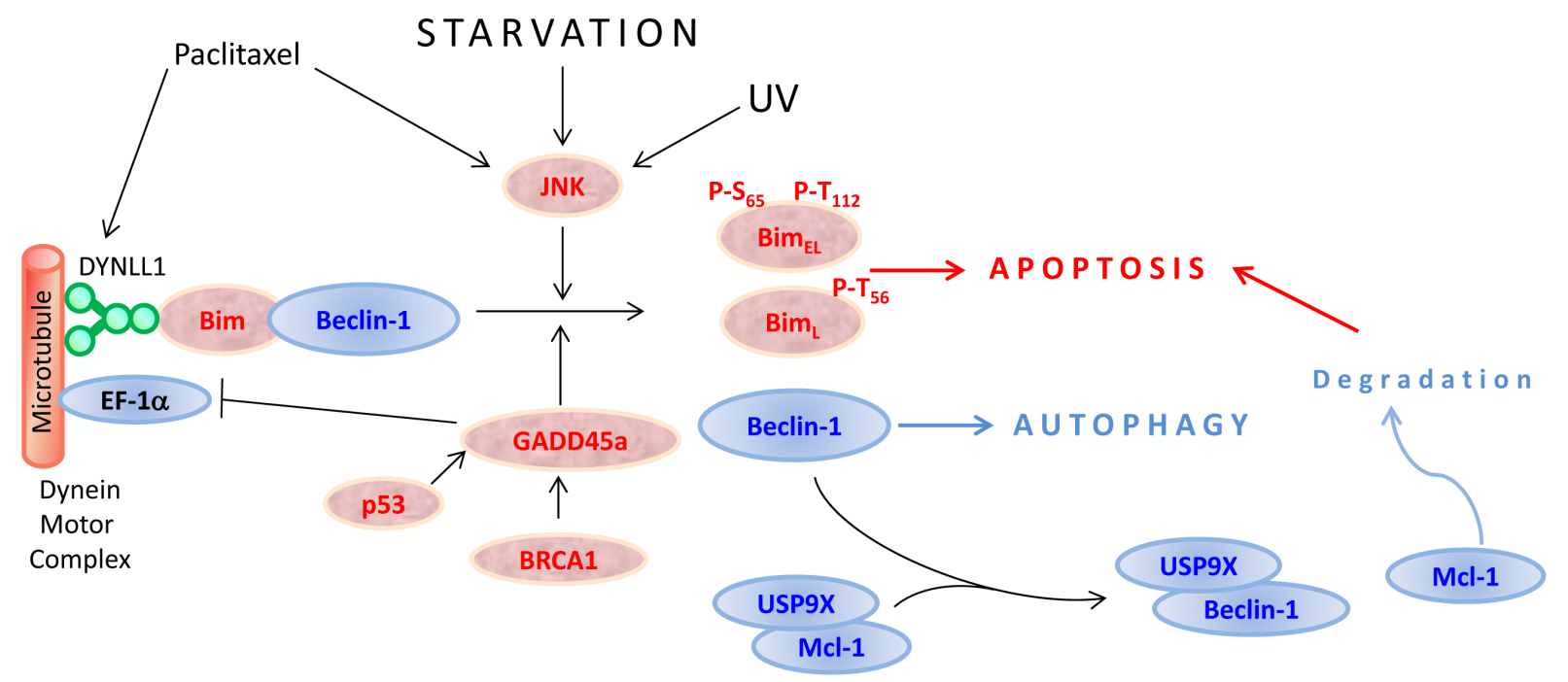

Figure 3: Bim at the cross-road between apoptosis and autophagy. In certain cell types such as epithelial and neuronal cells, $\mathrm{Bim}_{\mathrm{EL}}$ and $\mathrm{Bim}_{\mathrm{L}}$ are sequestered to the dynein motor complex through interaction with the dynein light chain DYNLL1. Bim also binds Beclin-1, thus preventing autophagy. Upon exposure to microtubule-disrupting agents such as paclitaxel, or stress-stimuli such as starvation or UV radiation that activate $\mathrm{JNK}, \mathrm{Bim}_{\mathrm{EL}}$ becomes phosphorylated at Ser65 and Thr112 and Bim ${ }_{\mathrm{L}}$ at Thr56, leading to their release from the microtubule and mitochondrial translocation where the intrinsic apoptotic pathway is activated. Simultaneously, Beclin-1 is released from Bim resulting in induction of autophagy. Beclin-1 also competes with Mcl-1 for the USP9X deubiquitinase resulting in enhanced Mcl-1 degradation, thereby tipping the Bim/Mcl-1 balance towards Bim-induced apoptosis. In addition, the p53- and BRCA1-regulated stress protein GADD45a promotes Bim dissociation from the microtubules. GADD45a also inhibits the function of the microtubule-severing protein EF-1 $\alpha$. 
apoptotic potential (see Section 1.2). Translation of Bim mRNA is negatively regulated by a series of microRNAs (e.g., miR-9, -181a, -17 92, -25, -32, -221/222 and -301a) and RNA-binding proteins (e.g., Hsc70 and Hsp27) (Figure 6). The activity and stability of the Bim protein are tightly regulated at the post-translational level (Figure 7). In healthy cells, Bim can be sequestered in its inactive form to the microtubular cytoskeleton or exist as inactive heterodimers with anti-apoptotic Bcl-2 family members sequestered to the mitochondria. Upon apoptosis induction, the sequestered Bim is released from these cellular stores to promote apoptosis. Another major regulatory mechanism of Bim-dependent apoptosis is driven by phosphorylation. Phosphorylation may either increase its activity (e.g., by JNK), or promote its degradation (e.g., by ERK/MAPK) thereby antagonizing apoptosis.

\subsection{Transcriptional regulation of Bim}

A 800-bp region upstream of exon 1 contains elements for the control of Bim transcription [17]. Basal Bim mRNA levels were observed in most normal tissues [3, 6]. Binding sites for several transcription factors including FoxO, c-Myb and c-Jun are present in the bim promoter [17, 116-118]. Mutation of either the FoxO, c-Myb or c-Jun sites abolished induction of a bim promoter-derived reporter in response to nerve growth factor (NGF) deprivation [116]. This suggests that the bim promoter acts as a coincidence detector that optimally responds to the simultaneous activation of the the three different pro-apoptotic transcriptional pathways. Such a mechanism provides a "fail-safe" that prevents neurons from dying by accidental activation of any single pathway [116]. In addition to the proximal bim promoter, the first intron contains additional regulatory sequences that respond to NGF deprivation [117, 119]. This intron contains FoxO and Myb binding sites $[116,117]$. Xie et al. [120] observed that early growth response 1 (Egr-1), rather than c-Jun, FoxO or c-Myb, transactivates Bim expression in cerebellar granule neurons. A putative Egrbinding sequence was found between nucleotides -56 and -47 upstream of the start site [120].

\subsubsection{Transcriptional Upregulation}

\subsubsection{FKHR-L1/FoxO3a}

The forkhead transcription factor FKHR-L1/FoxO3a was found to be essential for the transcriptional activation

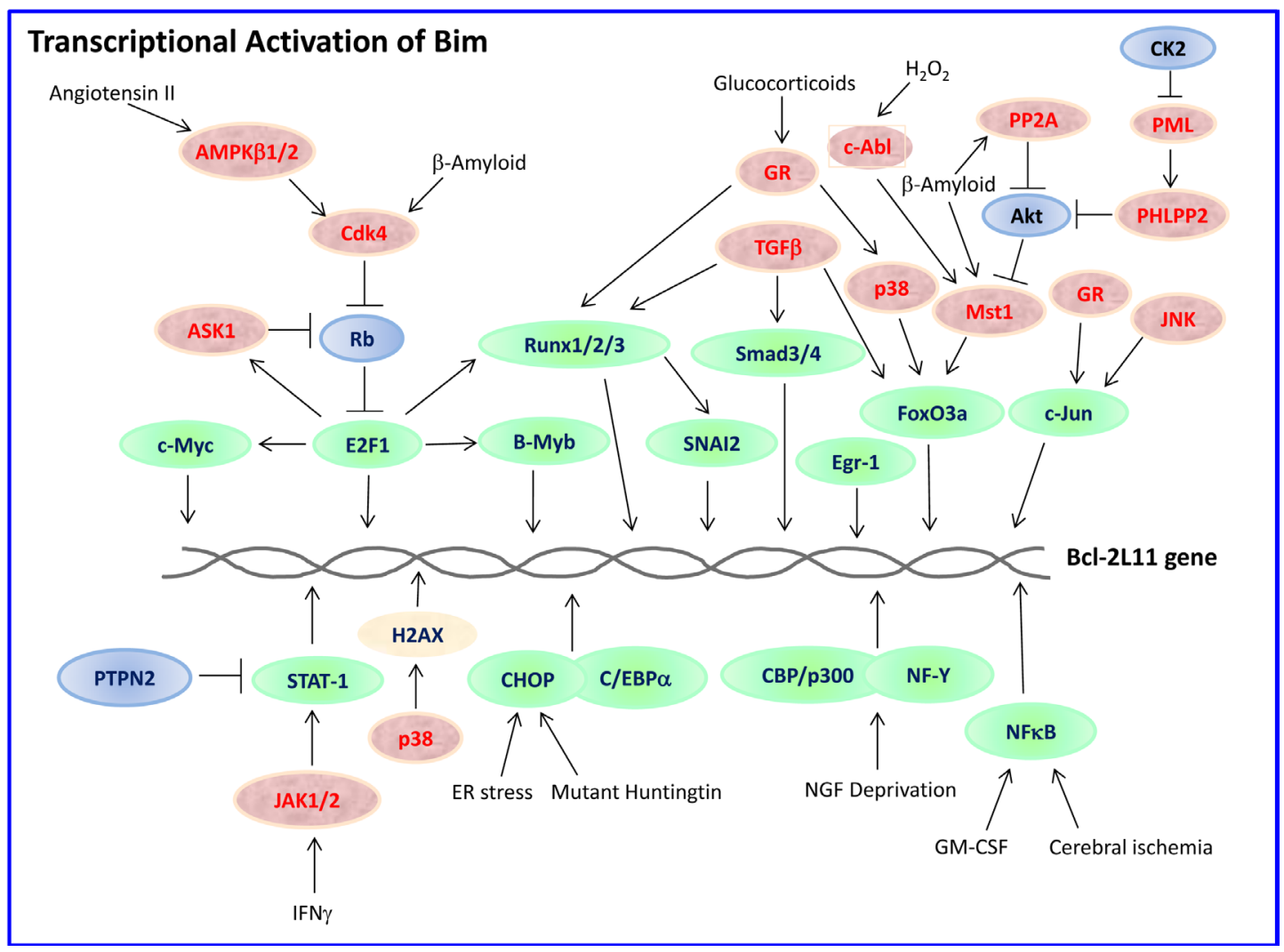

Figure 4: Transcriptional activation of Bim. Bim transcription can be upregulated by a whole range of transcription factors (outlined in green) whose activities are tightly regulated. Some of these pathways are cell-type specific and depend on the stimulus. Those proteins positively regulating the transcription factors are presented in red, while those inhibiting in blue. H2AX is a histone whose phosphorylation at Ser139 by p38 is important for Bim transcription. The different pathways are described in more detail in Section 3.1.1. 
Table 3: Bim in Stress-Induced Apoptosis.

\begin{tabular}{|c|c|c|}
\hline $\begin{array}{l}\text { Apoptotic } \\
\text { Stimulus }\end{array}$ & Remarks & References \\
\hline Anoikis & $\begin{array}{l}\text { - } \begin{array}{l}\text { Bim is strongly induced after epithelial cell detachment and } \\
\text { downregulation of Bim inhibited anoikis. }\end{array} \\
\text { - Overexpression of EGFR maintains ERK activity following detachment, } \\
\text { thus preventing Bim induction and anoikis. } \\
\text { - Overexpression of HER2 in breast cancer increases HIF-1 } \alpha \text { expression } \\
\text { and activates ERK and Akt, resulting in reduced Bim expression and } \\
\text { prevention of anoikis. } \\
\text { - Pokemon renders liver cells resistant to anoikis via suppression of Bim } \\
\text { transcription. } \\
\text { - Extracellular matrix metalloproteinase inducer (CD147) confers } \\
\text { resistance of breast cancer cells to anoikis through inhibition of Bim. } \\
\text { - Mesothelin and PINCH-1 promote ERK-mediated Bim degradation and } \\
\text { anchorage-independent growth. }\end{array}$ & $\begin{array}{l}{[210,224,} \\
488,489,490 \\
492,495-497]\end{array}$ \\
\hline $\begin{array}{l}\text { cAMP-induced } \\
\text { apoptosis }\end{array}$ & $\begin{array}{l}\text { - 8-CPT-cAMP treatment of T lymphoma cells induced Bim expression } \\
\text { and apoptosis. } \\
\text { - Cyclic AMP-dependent protein kinase A regulates apoptosis by } \\
\text { stabilizing Bim. } \\
\text { - Bim is required for cAMP-mediated apoptosis of double positive } \\
\text { thymocytes. }\end{array}$ & [341-343] \\
\hline AICAR & $\begin{array}{l}\text { - 5-Aminoimidazole-4-carboxamide riboside or acadesine (AICAR) } \\
\text { induces apoptosis in chronic lymphocytic leukemia through a } \\
\text { mechanism dependent on Bim and Noxa, but independent of AMPK } \alpha 1 \text {, } \\
\text { p53 and ATM. }\end{array}$ & [647] \\
\hline ER stress & $\begin{array}{l}\text { - Deficiency of Bim/Puma impedes ER-stress-induced Bax/Bak activation } \\
\text { and apoptosis. } \\
\text { - ER stress activates Bim by CHOP-C/EBPo-mediated transactivation } \\
\text { and phosphatase 2A-mediated dephosphorylation that leads to Bim } \\
\text { stabilization. }\end{array}$ & {$[35,69]$} \\
\hline $\begin{array}{l}\text { Coagulation } \\
\text { factor Fxa- } \\
\text { induced apoptosis }\end{array}$ & $\begin{array}{l}\text { - The coagulation factor Fxa that converts prothrombin into active } \\
\text { thrombin, induces apoptosis of epithelial tumor cells through a CREB- } \\
\text { and Bim-dependent mechanism. }\end{array}$ & [386] \\
\hline $\begin{array}{l}\text { Heat shock- } \\
\text { induced apoptosis }\end{array}$ & $\begin{array}{l}\text { - Bim-deficient cells are resistant to heat shock-induced cell death to a } \\
\text { similar extent as } \mathrm{Bax}^{-1-} \mathrm{Bak}^{-1 /} \text { cells. }\end{array}$ & [648] \\
\hline Ionomycin & - $\mathrm{CD}_{4}{ }^{+} 8^{+}$thymocytes from Bim KO mice were resistant to ionomycin. & {$[2]$} \\
\hline $\begin{array}{l}\text { Serum- } \\
\text { withdrawal- } \\
\text { induced apoptosis }\end{array}$ & $\begin{array}{l}\text { - Serum withdrawal induces Bim expression and apoptosis of CCl39 } \\
\text { fibroblasts. Both processes were prevented by thrombin through } \\
\text { activation of the Raf-MEK-ERK } 1 / 2 \text { and PI3K pathways. A protease- } \\
\text { activated receptor } 1 \text { (PAR1) agonist peptide also protected cells from } \\
\text { serum-withdrawal-induced apoptosis. }\end{array}$ & {$[376]$} \\
\hline $\begin{array}{l}\text { UV-induced } \\
\text { apoptosis }\end{array}$ & - Knockdown of Bim inhibited UV-induced cell death of COS-7 cells. & {$[60]$} \\
\hline
\end{tabular}

of Bim, especially after cytokine withdrawal and various chemotherapeutic agents $[118,121,122]$. Dijkers et al. [122] demonstrated that cytokine withdrawal led to FoxO3a activation and Bim induction in T lymphocytes accompanied by apoptosis. Cytokines promote lymphocyte survival by inhibiting the activity of FoxO3a, thus preventing Bim expression [122]. Overexpression of FoxO transcription factors induces Bim expression and promotes death of sympathetic neurons in a Bim-dependent manner [117]. In neuroblastoma cells, the activation of FoxO3 triggers the intrinsic death pathway via induction of Bim and Noxa [123]. Inhibition of Breakpoint cluster regionAbelson murine leukaemia (Bcr-Abl) kinase by STI571 in Bcr-Abl expressing cells results in FoxO3a activation, induction of Bim expression and apoptosis [121]. The microtubule interfering drug paclitaxel induced Bim expression in breast cancer cells that expressed high basal levels of FoxO3a, but not in those with low basal FoxO3a levels [118]. Knockdown of FoxO3a prevented Bim induction and apoptosis induced by paclitaxel [118]. In neuroblastoma, brain-derived neurotrophic factor (BDNF) activates the PI3K/Akt pathway resulting in suppression 
Table 4: Bim in Diabetes.

\begin{tabular}{|c|c|c|}
\hline $\begin{array}{l}\text { Apoptotic } \\
\text { Stimulus }\end{array}$ & Remarks & References \\
\hline $\begin{array}{l}\text { Diabetes- } \\
\text { susceptibility } \\
\text { genes }\end{array}$ & 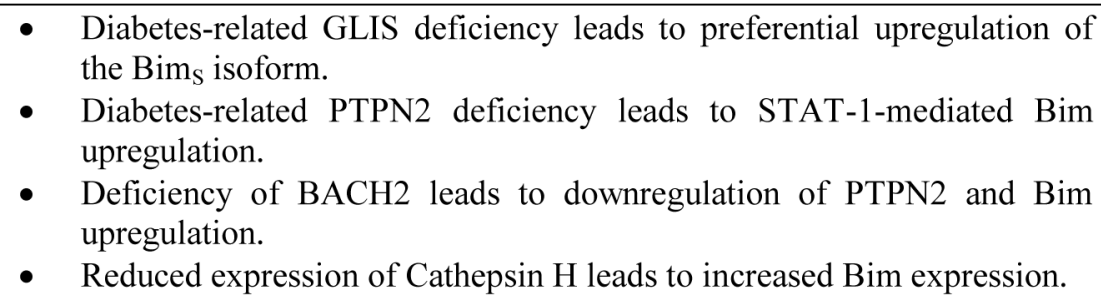 & $\begin{array}{l}{[183,233} \\
454,455]\end{array}$ \\
\hline $\begin{array}{l}\text { Pdx-1- } \\
\text { haploinsufficiency }\end{array}$ & $\begin{array}{l}\text { - The increased apoptosis of } \beta \text {-cells in Pdx-1-haploinsufficient mice could } \\
\text { be prevented by simultaneous knockdown of Bim. }\end{array}$ & {$[456]$} \\
\hline IRS2-deficiency & - $\quad$ Bim mediates $\beta$-cell death in IRS2-deficient mice. & [457] \\
\hline $\begin{array}{l}\text { Non-obese } \\
\text { diabetic (NOD) } \\
\text { mice }\end{array}$ & $\begin{array}{l}\text { - Decreased thymic deletion associated with diminished induction of Bim } \\
\text { when encountering high-avidity autoantigen. }\end{array}$ & [452] \\
\hline $\begin{array}{l}\text { Glucose } \\
\text { cytotoxicity of } \\
\text { insulin-producing } \\
\beta \text {-cells }\end{array}$ & $\begin{array}{l}\text { - Langerhans' islets lacking Bim or Puma were protected from glucose } \\
\text { cytotoxicity. } \\
\text { - High glucose induces the expression of CHOP that co-operates with } \\
\text { FoxO3a to regulate Bim and Puma expression. }\end{array}$ & $\begin{array}{l}{[181,450} \\
451,649]\end{array}$ \\
\hline $\begin{array}{l}\text { Pro-inflammatory } \\
\text { cytokines }\end{array}$ & $\begin{array}{l}\text { - Bim induced in Langerhans' islets by pro-inflammatory cytokines } \\
\text { promotes } \beta \text {-cell death. }\end{array}$ & {$[183,188]$} \\
\hline Thapsigargin & $\begin{array}{l}\text { - Loss of Bim or Puma partially protects Langerhans' islets from the ER } \\
\text { stressor thapsigargin. }\end{array}$ & {$[450]$} \\
\hline
\end{tabular}

of FoxO3a activity and Bim induction, thereby preventing paclitaxel-induced apoptosis [124]. Simultaneous activation of the MEK/ERK pathway further reduced Bim expression at the protein level [124]. FoxO3 binds to a FoxO-binding site (FHRE) within the bim promoter [118, 121]. FoxO3a acts in concert with the Activator protein-1 (AP-1) transcription factor [116, 117, 125].

High expression levels of FoxO3a correlated with long-term survival in breast cancer patients [126], and nuclear localization of FoxO3a is associated with longer luminal-like breast cancer survival and longer distant metastasis free interval [127]. FoxO3a expression was lower in nasopharyngeal carcinoma than in the normal nasopharyngeal tissues [128]. Nasopharyngeal carcinoma patients with low FoxO3a and high Hypoxia-inducible factor $1 \alpha$ (HIF-1 $\alpha$ ) expression had poorer prognosis than patients with high FoxO3a and low HIF-1 $\alpha$ levels [128]. Low levels of FoxO factors are associated with poor prognosis in liver cancer [129] and gastric adenocarcinoma [130]. Prostate cancer with increasing Gleason grade showed marked cytoplasmic accumulation of FoxO3a, in contrast to exclusive nuclear accumulation in benign prostate cells [131]. Thus, FoxO factors act as tumor suppressors that are frequently downregulated in advanced cancers.

Since FoxO3a is an important regulator of Bim, factors affecting FoxO3a expression have direct effect on tumorigenesis. For instance, ERK phosphorylates FoxO3a at Ser294, Ser344 and Ser425, which leads to
Mdm2-mediated degradation of FoxO3a via the ubiquitinproteasome system [132]. Sirtuin 1 (SIRT1) deacetylates FoxO3a leading to its ubiquitination and degradation, thus protecting against oxidative stress-induced apoptosis of endothelial cells [133]. Similarly, IкB kinase (IKK) and Akt (PKB) phosphorylate and cause proteolysis of FoxO3a [134, 135]. Akt phosphorylation of FoxO3a leads to cytoplasmic sequestration of $\mathrm{FoxO} 3 \mathrm{a}$ to $14-3-3$ proteins, thereby preventing the transcription of genes required for apoptosis [136] Akt phosphorylates FoxO3a at Thr32 and Ser253, while the Serum- and glucocorticoid-inducible kinase (SGK) phosphorylates it at Thr32 and Ser315. Cytoplasmic FoxO3a correlated with expression of IKK $\beta$ or phosphorylated Akt in many tumors and was associated with poor survival in breast cancer [134]. Inhibition of both the mTOR and MEK/ERK pathways led to increased nuclear accumulation and activation of FoxO3a that promoted differentiation and reduced tumorigenicity of glioblastoma cancer stem-like cells [137]. p38 MAPK phosphorylates FoxO3a at Ser7 leading to its nuclear accumulation [138], and induction of Bim expression [139].

In a recent publication, Chatterjee et al. [140] described an additional level of FoxO3a/Bim regulation (Figure 4). Namely, the Casein kinase II (CK2), which is activated under various stress conditions and is abnormally deregulated in many human malignancies, indirectly suppresses FoxO3a activity by promoting phosphorylation of the Promyelocytic leukemia (PML) 
Table 5: Bim in Liver Pathophysiology.

\begin{tabular}{|c|c|c|}
\hline $\begin{array}{l}\text { Apoptotic } \\
\text { Stimulus }\end{array}$ & Remarks & References \\
\hline $\begin{array}{l}\text { Acetaminophen- } \\
\text { induced liver } \\
\text { damage }\end{array}$ & $\begin{array}{l}\text { - Acetaminophen (paracetamol) induced Bim expression in hepatocytes } \\
\text { through a JNK-dependent manner. } \\
\text { Bim-deficient mice were protected from acetaminophen-induced liver } \\
\text { damage. }\end{array}$ & [464] \\
\hline $\begin{array}{l}\text { Hepatocyte } \\
\text { lipoapoptosis }\end{array}$ & $\begin{array}{l}\text { - Free saturated fatty acids induce lipoapoptosis of hepatocytes through } \\
\text { FoxO3a-dependent Bim upregulation and JNK-mediated Bim activation. } \\
\text { Free fatty acids induce the expression of PP2A responsible for FoxO3a } \\
\text { activation. } \\
\text { - Palmitic acid induces degradation of Keap-1, resulting in JNK-mediated } \\
\text { upregulation of Bim. }\end{array}$ & $\begin{array}{l}466,467, \\
469]\end{array}$ \\
\hline $\begin{array}{l}\text { Inflammation- } \\
\text { induced } \\
\text { hepatocyte } \\
\text { apoptosis }\end{array}$ & $\begin{array}{l}\text { - Overactivation of the immune system can lead to apoptotic death of } \\
\text { hepatocytes that is mediated by TNF } \alpha \text {-induced JNK-mediated activation } \\
\text { of Bim and caspase } 8 \text {-mediated activation of Bid. }\end{array}$ & {$[468]$} \\
\hline $\begin{array}{l}\text { Virus-induced } \\
\text { hepatitis }\end{array}$ & $\begin{array}{l}\text { - Bim is involved in the elimination of liver-activated virus-specific } \mathrm{T} \\
\text { cells following HBV/HCV and LCMV infections. } \\
\text { LCMV leads to persistence of cytotoxic } \mathrm{T} \text { cells that induce hepatocyte } \\
\text { apoptosis by a Bim-dependent mechanism. } \\
\text { Bim }{ }^{-/-} \text {hepatocytes showed reduced sensitivity to } \mathrm{T} \text { cell-induced } \\
\text { apoptosis. } \\
\text { Bim is upregulated in HBV-specific } \mathrm{CD} 8^{+} \mathrm{T} \text { cells from patients with } \\
\text { chronic HBV infection, leading to early contraction of the immune } \\
\text { response. }\end{array}$ & $\begin{array}{l}{[353,472,} \\
474,475]\end{array}$ \\
\hline
\end{tabular}

protein at Ser517, resulting in its ubiquitin-mediated proteasomal degradation [141]. PML is required for the functional interaction of activated phosphorylated Akt and its phosphatases (e.g., PHLPP2) inside the nucleus, that leads to inactivation of Akt [142] and proper functioning of FoxO3a [140]. In the absence of PML, Akt is aberrantly activated, leading to nuclear exclusion of FoxO3a. As a consequence, the FoxO3 target genes p $27^{\mathrm{Kip} 1}, \mathrm{p} 21^{\mathrm{Cip} 1}$ and Bim are downregulated, while VEGF gene expression, which is suppressed by FoxO3a [143], becomes upregulated [140, 142]. Thus, CK2 antagonizes apoptosis by relieving the negative effect of PML on Akt, that in turns negatively regulate FoxO3a-mediated transcription of Bim.

Although FoxO3a is a central regulator of Bim expression after growth factor and cytokine withdrawal and some chemotherapeutic agents $[117,118,121,122$, 144], mice with mutated FoxO-binding sites within the bim promoter had a normal hematopoietic system, and showed similar death rates of cytokine-dependent haematopoietic cells as wild-type mice [145]. This study suggests that the direct transcriptional induction of Bim by FoxO transcription factors is not critical for Bim's pro-apoptotic function in the hematopoietic system, at least under normal homeostasis. This FoxO independence could be due to already high basal Bim expression in these cells $[63,64,145]$. The same research group showed that endothelial cell apoptosis in response to VEGF-A inhibitors is Bim-dependent, but could proceed normally in mice lacking FoxO-binding sites in the bim promoter [146]. As FoxO3 KO mice show altered hematopoietic homeostasis [147-149], it is likely that FoxO3a may affect Bim transcription through interaction with other transcription factors, or that there is a compensation for FoxO3.

\subsubsection{NF-Y}

The heterotrimeric transcription factor NF-Y is required for Bim induction in neurons following NGF withdrawal [150]. The transcriptional coactivators CBP and p300 interact with NF-Y and FoxO3a and bind to the CCAAT box (ICB) of the bim promoter [150]. The amount of $\mathrm{CBP} / \mathrm{p} 300$ bound to the bim promoter increases after NGF deprivation, and inhibition of $\mathrm{CBP} / \mathrm{p} 300$ activity reduces Bim induction [150]. Thus, NF-Y cooperates with FoxO3a to recruit $\mathrm{CBP} / \mathrm{p} 300$ to the bim promoter [150]. The C subunit of NF-Y was found to be upregulated in hippocampal CA1 neurons following cerebral ischemia concomitant with the upregulation of Bim [151].

\subsubsection{Smad2/3/4}

Overexpression of Smad3, a transcription factor activated by TGF $\beta$, increased the expression of Bim in B lymphocytes [152]. Activation of the pro-survival transmembrane glycoprotein CD40 abrogated TGF $\beta$ mediated Bim induction and apoptosis [152]. Smad3 was also found to be involved in the transcriptional activation of Bim in human gastric carcinoma cells when 
Table 6. Bim in the Cancer Context.

\begin{tabular}{|c|c|c|}
\hline $\begin{array}{l}\text { Apoptotic } \\
\text { Stimulus }\end{array}$ & Remarks & References \\
\hline Granzyme B & $\begin{array}{l}\text { - Bim-deficient 3T9 transformed mouse embryonic fibroblasts are } \\
\text { resistant to Granzyme B-induced apoptosis. }\end{array}$ & {$[650]$} \\
\hline IFN $\alpha$ & $\begin{array}{l}\text { IFNa-induced apoptosis of multiple myeloma cells depends on Jak1 and } \\
\text { Bim. }\end{array}$ & [601] \\
\hline $\begin{array}{l}\text { Melatonin } \\
\text { (N-acetyl-5- } \\
\text { methoxy- } \\
\text { tryptamine) }\end{array}$ & $\begin{array}{l}\text { - Melatonin induces apoptosis of HepG2 hepatoma cells through FoxO3a- } \\
\text { mediated induction of Bim. } \\
\text { Melatonin induces apoptosis of human renal Caki cells by upregulating } \\
\text { Bim through induction of SP-1 and E2F1-mediated transcription and } \\
\text { inhibition of proteasomal activity. }\end{array}$ & {$[318,320]$} \\
\hline $\begin{array}{l}\text { c-Myc induced } \\
\text { apoptosis }\end{array}$ & - Bim mediates c-Myc induced apoptosis in solid tumors. & {$[191]$} \\
\hline $\begin{array}{l}\text { TGF } \beta \text {-induced } \\
\text { apoptosis }\end{array}$ & 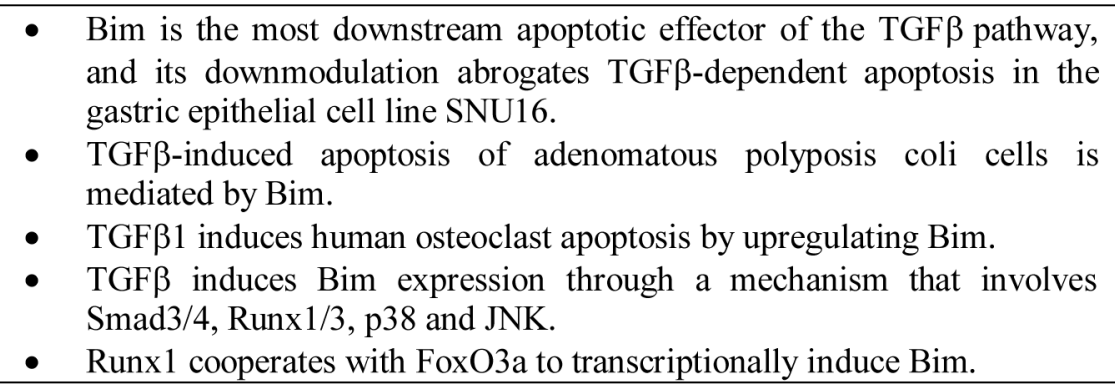 & $\begin{array}{l}{[42,152,153,} \\
165,166,553- \\
555]\end{array}$ \\
\hline
\end{tabular}

simultaneously exposed to both TGF $\beta$ and TNF $\alpha$ [153]. Under these conditions the Bim protein was stabilized by a JNK-dependent mechanism [153]. TGF $\beta$-induced apoptosis of human hepatoma cells was also mediated by a Smad3/Smad4-mediated upregulation of Bim [154]. TGF $\beta$ also activates FoxO3 dephosphorylation of Thr32, leading to its nuclear translocation [155]. These authors further showed that the TGF $\beta$-mediated upregulation of Bim and apoptosis was dependent on Casein kinase I $\varepsilon(\mathrm{CKI} \varepsilon)$ [155]. Moreover, the TGF $\beta$-activated FoxO3 cooperated with Smad2/3 to mediate Bim upregulation and apoptosis [155].

\subsubsection{4. c-Jun and Runx1-3}

The JNK/c-Jun pathway was found to be important for Bim upregulation in neurons following survival signaling withdrawal $[116,125,156]$. However, activity deprivation-dependent induction of Bim during apoptosis in cerebellar granule neurons occurred independently of JNK/c-Jun activation [157]. p38 MAPK that is important for the upregulation of Bim in CCRF-CEM T-ALL cells in response to glucocorticoids [158], acts upstream to the transcription factors Runx2 and c-Jun that positively activate the transcription of Bim [159]. c-Jun was upregulated in glucocorticoid-sensitive, but not glucocorticoid-resistant CCRF-CEM T-ALL cells, which correlated with Bim induction [160]. It should be noted that the requirement for $\mathrm{p} 38$ in the upregulation of Bim was only observed in certain leukemic cell types, while it was dispensable for Bim expression in others [63]. Another protein kinase that might contribute to Bim induction is GSK3 [161].
Runx2 may affect Bim expression through the zinc finger transcription factor Snail family zinc finger 2 (SNAI2). Runx2 induces epithelial-mesenchymal transition (EMT) through induction of SNAI expression [162], which binds to the bim promoter and promotes Bim expression [163]. Runx3 is responsible for the transcriptional upregulation of Bim by TGF $\beta$ [164]. Lack of Runx 3 functions is causally related to the genesis and progression of gastric cancer [164]. TGF $\beta$ also induces the expression of the transcription factor Runx 1 that binds to FoxO3, to enhance its ability to transcribe Bim [165]. A putative forkhead binding element (FHBE) located at position -168 to -174 from the transcription start site is involved in Bim induction by Runx1 and FoxO3a [165]. In addition, TGF $\beta$ indirectly increases Bim protein stability through Smad3-dependent induction of the mitogen-activated protein kinase (MAPK) phosphatase MKP2/DUSP4 that reduces the activity of ERK [166]. As ERK phosphorylates Bim and promotes its proteasomal degradation (see Section 3.3.1.1), inhibition of ERK leads to increased Bim stability. Thus, TGF $\beta$ increases Bim expression both at the transcriptional and post-translation levels.

Double positive thymocytes overexpressing the Runt domain of Runx1 expressed elevated levels of Bim [167]. As the Runt domain is expected to act as a dominant negative form of Runx1, Abe et al. [167] suggested that Runx1 suppresses the apoptotic sensitivity of double positive thymocytes in the context of $\mathrm{T}$ cell receptor signaling. 
Table 7: Bim in Drug-Induced Apoptosis.

\begin{tabular}{|c|c|c|}
\hline $\begin{array}{l}\text { Apoptotic } \\
\text { Stimulus }\end{array}$ & Remarks & References \\
\hline $\begin{array}{l}\text { ALK and c-Met } \\
\text { inhibitors }\end{array}$ & $\begin{array}{l}\text { - The ALK inhibitor TAE684 induces apoptosis in lung cancer cells } \\
\text { through upregulation of Bim and downregulation of survivin. } \\
\text { - The dual ALK and c-Met inhibitor Crizotinib induces apoptosis in c- } \\
\text { Met-amplificated lung cancer cells and gastric cancer cells through } \\
\text { upregulation of Bim. } \\
\text { - When combined with EGFR inhibitors, the dual ALK and c-Met } \\
\text { inhibitor CM-118 induced apoptosis of c-Met amplified NSCLC cells } \\
\text { through Bim upregulation and Mcl-1 downregulation. }\end{array}$ & {$[623,625-627]$} \\
\hline $\begin{array}{l}\text { BH3 mimetics } \\
\text { (e.g., ABT-737, } \\
\text { ABT-263 and } \\
\text { ABT-199) }\end{array}$ & $\begin{array}{l}\text { - The apoptosis induction of ovarian cancer by ABT-737 was dependent } \\
\text { on Bim expression. } \\
\text { ABT-737-mediated release of Bak couldn't induce apoptosis unless Bim } \\
\text { associates with oligomeric Bak to promote its conversion to a } \\
\text { membrane-inserted pore. } \\
\text { - The pro-apoptotic effect of ABT-737 in CLL depends on sufficient } \\
\text { amount of Bcl-2 that tonically sequesters the pro-apoptotic Bim protein. } \\
\text { - Lung cancer with EGFR mutation and high Bcl-2 expression could be } \\
\text { sensitized to cell death by a combination of erlotinib and ABT-737 that } \\
\text { was dependent on Bcl-2 primed with Bim. } \\
\text { - Primary B-ALL cells expressing high levels of Bcl-2 exhibited great } \\
\text { sensitivity to ABT-263 and ABT-199 } \\
\text { BH3 profiling of lymphoma cells identifies cells dependent on Bcl-2 and } \\
\text { predicts sensitivity to ABT-737. } \\
\text { Hepatocyte apoptosis induced by ABT-737 was completely prevented in } \\
\text { Bim/Bid double knockout mice. }\end{array}$ & $\begin{array}{l}{[78,309,515} \\
572,577,585 \\
651]\end{array}$ \\
\hline $\begin{array}{l}\text { Bortezomib } \\
\text { (Velcade) }\end{array}$ & $\begin{array}{l}\text { - Bortezomib-induced apoptosis of multiple myeloma cells was prevented } \\
\text { upon knockdown of Bim. } \\
\text { Bortezomib sensitized prostate cancer cells to TRAIL-induced apoptosis } \\
\text { through a mechanism dependent on Bim. }\end{array}$ & {$[485,507]$} \\
\hline $\begin{array}{l}\text { Etoposide and } \\
\text { Doxorubicin }\end{array}$ & $\begin{array}{l}\text { - Etoposide- and doxorubicin-induced apoptosis of neuroblastoma cells } \\
\text { was dependent on FoxO3-mediated Bim expression and ROS production } \\
\text { that could be prevented by Bcl-xL. }\end{array}$ & [57] \\
\hline Flt3 inhibitors & $\begin{array}{l}\text { - The Flt3 inhibitors AG1295 and PKC412 induced apoptosis of acute } \\
\text { myeloid leumemia cells that was dependent on FoxO3a-mediated Bim } \\
\text { induction and inactivation of the PI3K/Akt pathway. }\end{array}$ & {$[652]$} \\
\hline Glucocorticoids & 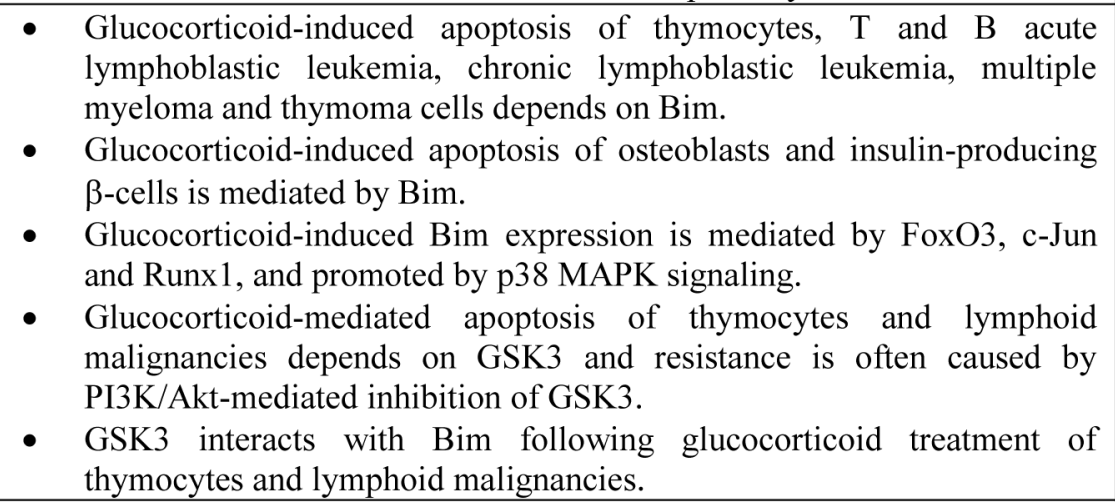 & $\begin{array}{l}{[14,21,49} \\
63,158,341 \\
458,517-521 \\
523,653]\end{array}$ \\
\hline $\begin{array}{l}\text { Histone } \\
\text { deacetylase } \\
\text { (HDAC) } \\
\text { inhibitors }\end{array}$ & $\begin{array}{l}\text { - The HDAC inhibitor suberic bishydroxamate (SBHA) upregulated Bim, } \\
\text { Bax and Bak in human melanoma cells. } \\
\text { The HDAC inhibitor Trichostatin A restored histone acetylation, with } \\
\text { concomitant upregulation of Bim. }\end{array}$ & $\begin{array}{l}{[32,242,245,} \\
272,503,539]\end{array}$ \\
\hline
\end{tabular}




\begin{tabular}{|c|c|c|}
\hline & $\begin{array}{l}\text { - Trichostatin A suppressed miR-106b 93 25 expression through } \\
\text { downregulation of c-Myc, thereby increasing Bim expression and } \\
\text { apoptosis in human endometrial cancer cells. } \\
\text { The HDAC inhibitor vorinostat increased Bim expression and sensitized } \\
\text { EGFR-mutant non-small-cell lung cancer (NSCLC) to the tyrosine } \\
\text { kinase inhibitor gefitinib. }\end{array}$ & \\
\hline $\begin{array}{l}\text { Imatinib (STI571) } \\
\text {-induced } \\
\text { apoptosis }\end{array}$ & 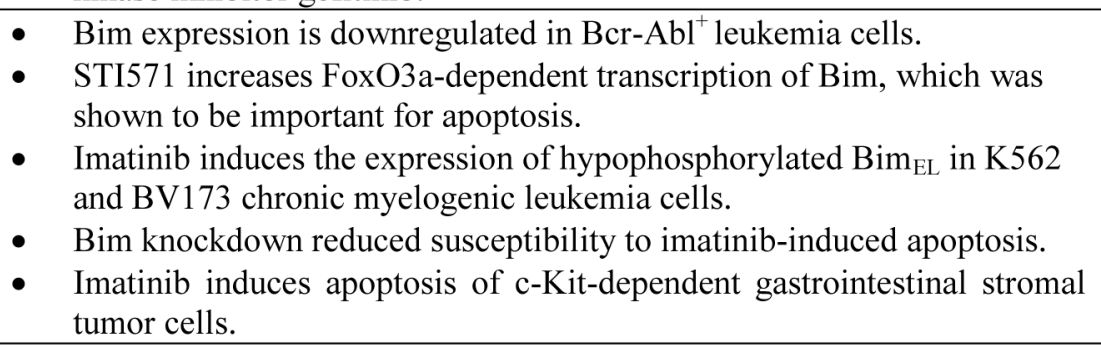 & $\begin{array}{l}{[121,248} \\
249,304,305 \\
501,549,646]\end{array}$ \\
\hline $\begin{array}{l}\text { Erlotinib-induced } \\
\text { apoptosis }\end{array}$ & $\begin{array}{l}\text { - The EGFR kinase inhibitor Erlotinib increased Bim expression in lung } \\
\text { cancer cells sensitive to the drug, but not in resistant cells. } \\
\text { - Bcl-2 inhibits the cell death induced by erlotinib. }\end{array}$ & {$[308,309]$} \\
\hline $\begin{array}{l}\text { Gefitinib-induced } \\
\text { apoptosis }\end{array}$ & $\begin{array}{l}\text { - Bim is involved in gefitinib-induced apoptosis in sensitive EGFR- } \\
\text { mutant cancer cells. } \\
\text { T790M mutation in EGFR prevented gefitinib-induced upregulation of } \\
\text { Bim and apoptosis. } \\
\text { T790M mutated cells responded with Bim upregulation and apoptosis } \\
\text { when using the irreversible tyrosine kinase inhibitor CL-387,785. }\end{array}$ & {$[306,307]$} \\
\hline $\begin{array}{l}\text { Lapatinib-induced } \\
\text { apoptosis }\end{array}$ & $\begin{array}{l}\text { - Lapatinib treatment of sensitive, but not resistant, HER2 }{ }^{+} \text {breast cancer } \\
\text { cells led to increased Bim expression. } \\
\text { - Downregulation of PTK6 induced apoptosis of resistant breast cancer } \\
\text { cells through a Bim-dependent mechanism. }\end{array}$ & {$[500]$} \\
\hline $\begin{array}{l}\text { Mutant B-Raf } \\
\text { inhibitor-induced } \\
\text { apoptosis }\end{array}$ & $\begin{array}{l}\text { - Inhibition of B-Raf }{ }^{\mathrm{V} 600 \mathrm{E}} \text { in melanoma cells by PLX4720 led to induction } \\
\text { of the three Bim isoforms Bim }{ }_{\mathrm{EL}}, \mathrm{Bim}_{\mathrm{L}} \text {, and } \mathrm{Bim}_{\mathrm{S}} \text {, but the increase in } \\
\text { Bim } \mathrm{S} \text { was the most profound. } \\
\text { The splicing factor SRp55 was responsible for the increased Bim } \\
\text { splicing. } \\
\text { Dual treatment of PTEN-negative melanoma cells with PLX4720 and a } \\
\text { PI3K inhibitor enhanced Bim expression and apoptosis. } \\
\text { - Combining the B-Raf inhibitor vemurafenib with the MEK inhibitor } \\
\text { trametinib increased Bim expression and apoptosis. }\end{array}$ & $\begin{array}{l}{[235,541,} \\
633]\end{array}$ \\
\hline $\begin{array}{l}\text { Paclitaxel } \\
\text { (Taxol)-induced } \\
\text { apoptosis }\end{array}$ & $\begin{array}{l}\text { - Paclitaxel induced FoxO3a and Bim expression in MCF-7 cells, both } \\
\text { involved in apoptosis. } \\
\text { Knockdown of Bim decreased the susceptibility of non-small-cell lung } \\
\text { cancer (NSCLC) cells to paclitaxel-mediated killing. } \\
\text { siRNA silencing of Bim reduced sensitivity of K562 cells to Taxol- } \\
\text { induced cell death. }\end{array}$ & $\begin{array}{l}{[118,346,} \\
482,654,655]\end{array}$ \\
\hline PP2A activator & $\begin{array}{l}\text { - The PP2A activator FTY720 induces apoptosis of chronic myelogenic } \\
\text { leukemia cells through activation of Bim and Bid. } \\
\text { - FTY720 overcomes tyrosine kinase inhibitor resistance caused by Abl } \\
\text { kinase domain mutations. }\end{array}$ & [656] \\
\hline
\end{tabular}

\subsubsection{E2F1}

E2F transcription factors are best known for their involvement in the timely activation of genes required for cell cycle progression. E2F activity is negatively regulated through its interaction with the retinoblastoma $(\mathrm{Rb})$ tumor suppressor. Human tumors with inactive $\mathrm{Rb}$ pathway, often show deregulated E2F1 activity. Ectopic expression of E2F1 resulted in induction of Bim expression and apoptosis [168]. Also the BH3-only proteins PUMA, Noxa and Hrk/DP5 were upregulated by E2F1 [168]. Inhibitors of histone deacetylases (HDACi) were shown to target the $\mathrm{Rb} / \mathrm{E} 2 \mathrm{~F} 1$ pathway for apoptosis induction through activation of Bim [169]. Cancer cells with elevated E2F1 activity were highly sensitive to HDACi-induced cell death [169]. E2F1 also transactivates apoptosis signalregulated kinase 1 (ASK1) that further enhances E2F1mediated Bim transcription through inhibition of $\mathrm{Rb}$ [170]. ASK1 knockdown led to reduced E2F1-induced 
Bim transcription and reduced apoptosis in response to the HDAC inhibitor suberoylanilide hydroxamic acid (SAHA) [170].

Spinal cord injury led to strong induction of E2F1 and Bim followed by neuronal apoptosis that could be prevented by inhibition of E2F1 [171]. Angiotensin II, a key pro-apoptotic factor in fibrosis, mediates apoptosis in primary pulmonary artery endothelial cells through E2F1-mediated upregulation of Bim [172]. Angiotensin II regulates the association of AMP-regulated protein kinase $\beta 1 / 2$ (AMPK $\beta 1 / 2)$ with cyclin-dependent kinase 4 (Cdk4) leading to hyperphosphorylation of $\mathrm{Rb}$ and the release of E2F1 for transcriptional activation [172].

E2F1 also induces the expression of the oncogenic polycomb histone methyltransferase enhancer of zeste homolog 2 (EZH2), that antagonizes the induction of Bim by E2F1 [173] as well as the transcription of Runx3 [174] that also upregulates Bim [164]. Thus, the apoptotic activity of E2F1 is restrained in human cancer by the concomitant induction of EZH2 and Bim [173]. Moreover, deregulated E2F1 activity in gastric cancer may confer resistance to TGF $\beta$-induced apoptosis through upregulation of the miR-106 25 cluster that targets Bim [175]. Thus, E2F1 has opposing effects on Bim expression.

Upregulated Bim expression in prostate and breast cancer cells was dependent on E2F1, where E2F1 silencing led to loss of Bim [176]. These authors identified eight endogenous E2F1-binding sites in the Bim promoter. However, FoxO3a didn't bind to the Bim promoter in these cancer cells [176]. Interestingly, Gogada et al. [176] observed that Bim silencing or microinjection of anti-Bim antibodies into the cell cytoplasm of breast cancer cells resulted in cell rounding, detachment, and subsequent apoptosis, suggesting that Bim might have a pro-survival role in addition to being pro-apoptotic. The low proapoptotic activity of Bim in the epithelial cancer cells was explained by sequestration of Bim to microtubules, Bcl-xL and Mcl-1 together with low expression of Bax and Bax and elevated expression of X-linked inhibitor of apoptosis protein (XIAP) that inhibits caspase 9 and caspase 3 [176]. Since Bim associates with the microtubule, it could be that Bim is important for stabilizing the cytoskeleton required for survival. Alteration in cytoskeleton integrity might affect mitochondrial respiration and apoptosis [177].

\subsubsection{CHOP-C/EBP $\alpha$}

Bim was found to be essential for ER stress-induced apoptosis in thymocytes, macrophages, and epithelial cells from breast and kidney [35]. In other cell types, e.g., neuroblastoma, colon carcinoma and mouse embryonal fibroblasts (MEFs), ER-stress-induced apoptosis can also be mediated by Puma and Noxa [178, 179]. ER stress activates Bim through two different pathways. One involves direct transcriptional activation by the $\mathrm{C} /$ EBP homologous protein (CHOP/GADD153)-C/EBP $\alpha$ transcription factors, while the other involves protein phosphatase 2A (PP2A)-mediated dephosphorylation, which prevents its ubiquitin-dependent proteasomal degradation [35]. Also, mutant huntingtin that is responsible for the selective loss of medium spiny neurons in the striatum of patients with Huntington's disease, upregulates both GADD153 and Bim ${ }_{\mathrm{EL}}$ [180]. Knockdown of $\mathrm{Bim}_{\mathrm{EL}}$ prevented mutant huntingtin-induced apoptosis [180]. In thapsigargin-treated MCF-7 cells, FoxO3a and FoxO1 undergo dephosphorylation after Bim induction, suggesting that FoxO3a is not important in this setting [35]. FoxO3a-deficient thymocytes are normally sensitive to thapsigargin and tunicamycin [35]. According to Gosh et al. [181], CHOP co-operates with FoxO3a to upregulate Bim and Puma in response to ER stress in cortical neurons. Co-immunoprecipitation studies showed that CHOP interacts with FoxO3a [181]. CHOP may also cooperate with Activating transcription factor 4 (ATF4) for transcriptional upregulation, as seen for Puma in cortical neurons [182].

\subsubsection{STAT-1}

The transcription factor Signal transducer and activator of transcription 1 (STAT-1) has been shown to be involved in the induction of Bim during TNF $\alpha$ - and IFN $\gamma$ induced pancreatic $\beta$-cell apoptosis [183]. IFN $\gamma$ induces Bim transcription [183], while TNF $\alpha$ activates JNK that phosphorylates, and thereby upregulates Bim protein expression by protein stabilization [184]. IFN $\gamma$ activates signal transduction pathways that involve the tyrosine Janus kinases JAK1 and JAK2, which phosphorylate and induce the dimerization of STAT-1 [185]. Silencing of Bim or STAT-1 protected $\beta$-cells from cytokineinduced apoptosis $[183,186]$. STAT-1 directly binds to the bim promoter in positions -686 to -385 [183]. This region contains two STAT-1 binding sites, TTCtacGAA and TTCttgGAA [183]. Knockdown of the PTPN2 phosphatase, a candidate gene for type 1 diabetes [187], led to increased phosphorylation and activation of STAT-1 in $\beta$-cells, and increased cytokine-induced Bim expression $[183,188]$. Diabetic retinopathy caused by high glucoseinduced apoptosis of retinal pericytes involved STAT-1mediated Bim expression [189]. TNF $\alpha$ was responsible for the high glucose-mediated activation of STAT-1, while Bim was responsible for the high glucose-induced ROS production [189]. STAT-1-dependent Bim transactivation was also observed in chronic lymphoblastic leukemia cells after exposure to IL-21, a gamma-chain receptor cytokine family member that promotes B cell apoptosis [190].

\subsubsection{8. c-Myc}

c-Myc is one of the most frequently overexpressed oncogenes in human cancer and promotes ectopic proliferation in many post-mitotic cells. However, deregulated c-Myc expression sensitizes the cells to apoptosis by a Bim-dependent mechanism [191]. c-Myc was found to bind to the bim promoter and promote Bim transcription [191, 192]. Bim induction by c-Myc was 
independent of the p53 tumor suppressor that is also activating by wild-type c-Myc [193]. Mutant c-Myc, however, was unable to induce Bim expression, while still activated the p53 pathway to a similar extent as wild-type c-Myc [193]. This may explain why tumors with mutant c-Myc are less prone to apoptosis, and mutant c-Myc is more oncogenic [193]. Of note, wild-type and mutant c-Myc were equally oncogenic in the absence of Bim [193], further emphasizing the role of Bim in antagonizing the oncogenic effects of c-Myc. Hemann et al. [193] further showed that Burkitt's lymphoma expressing wildtype c-Myc displayed substantially higher Bim levels than diffuse, large $\mathrm{B}$ cell lymphomas (DLBCL) without c-Myc translocation. In contrast, Burkitt's lymphomas with mutated c-Myc were usually Bim negative [193]. The differential effect of wild-type and mutant c-Myc on Bim expression may also explain the opposing effects described for c-Myc on glucocorticoid-induced apoptosis [194-198], a death process dependent on Bim [199]. Moreover, c-Myc may reduce Bim expression through induction of miR-17 92 [200-202] (see Section 3.2.2.2).

c-Myc was also found to be responsible for Bim overexpression in human epidermal growth factor receptor 2 (HER2)-overexpressing breast cancer cells [192]. c-Myc expression in these cells was dependent on mTORC1 activity [192]. A simultaneous elevation of Mcl-1 in naïve HER2-positive cancer cells antagonized the pro-apoptotic effect of Bim [192]. Reduction of Mcl-1 expression was sufficient to induce apoptosis of HER2-overexpressing cells, suggesting that cell survival/death is determined by the Mcl-1/Bim balance [192]. Similar findings were observed by Horiuchi et al. [203] in triple-negative breast cancer that overexpressed c-Myc. c-Myc overexpression led to increased expression of Bim and the anti-apoptotic proteins Bcl-2, Bcl-xL and Mcl-1 [203]. The elevated c-Myc expression sensitized the triple-negative breast cancer cells to CDK inhibition by either Purvalanol A or Dinaciclib that was dependent on Bim [203].

\subsubsection{Nrf2}

The nuclear factor erythroid-derived 2, like 2 (Nrf2) is a key regulator of the antioxidant defense system that is activated under hyperprolifeation conditions in the liver. Activated Nrf2 delays proliferation and induces apoptosis of hepatocytes in the regenerating liver through direct transcriptional upregulation of Bim [204].

\subsubsection{Transcriptional Repression}

\subsubsection{YY-1-RelA}

In multiple myeloma, Bim transcription is repressed by the Yin Yang 1 (YY1)-RelA (p65) complex [205]. RelA

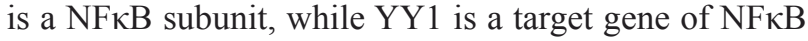
[205]. Deletion of either YY1 or RelA was sufficient to increase the expression level of Bim, with subsequent induction of apoptosis [205]. All three Bim isoforms, $\operatorname{Bim}_{E L}, B_{L}$ and $B_{S}$, were upregulated upon repression of either YY1 or RelA [205]. This research group further showed that YY1 and RelA need to be co-expressed for transcriptional repression of Bim [205]. YY1 and RelA bind within a region spanning between nucleotides -300 and -156 of the bim promoter [205]. However, under conditions of cerebral ischemia, RelA functioned as a transcriptional activator of Bim [206]. It could be that the interaction of YY1 with RelA is involved in switching RelA from being an activator to a repressor of the bim promoter.

\subsubsection{HoxB8}

In hematopoietic progenitor cells, Homeobox B8 (HoxB8) overexpression repressed Bim through a c-Mycdependent upregulation of miR-17 92 that binds to the 3'UTR of Bim mRNA [207]. HoxB8 overexpression immortalizes hematopoietic progenitor cells in a growth factor-dependent manner and co-operates with IL-3 to induce acute myeloid leukemia. Downregulation of HoxB8, in the presence of IL-3, caused cell-cycle arrest and apoptosis that was dependent on Bax and Bak, and in part, on Bim [207]. The requirement of miR-17 92 to suppress Bim expression by HoxB8 is evident in the strong selection against deletion of miR-17 92 in HoxB8immortalized myeloid cells [207].

\subsubsection{Spi-1/PU.1}

In erythroleukemia, the transcription factor Spi-1/ PU.1 inhibits apoptosis through transcriptional repression of Bim [208]. Spi-1 represses Bim transcription by binding to the bim promoter and by promoting trimethylation of histone 3 on Lys27 (H3K27me3, a repressive histone marker) on the bim promoter [208]. The Polycombrepressive complex 2 ( $\mathrm{PRC} 2)$ is responsible for the histone methylation [208]. A Spi-1 binding site was identified $0.3 \mathrm{~kb}$ upstream of the Bim transcriptional start site [208]. The two PRC2 subunits SUZ12 and the histone methyltransferase EZH2 interact with Spi-1 and bind to the bim promoter at the $\mathrm{H} 3 \mathrm{~K} 27 \mathrm{me} 3$ sites that are $0.9 \mathrm{~kb}$ distant from the Spi-1 binding motif [208]. The transcription cofactor Tripartite motif containing 33 (Trim33) is recruited to an enhancer upstream to the Bim gene by PU.1, leading to suppression of Bim expression and protection of $\mathrm{B}$ lymphoblastic leukemia from apoptosis [209].

\subsubsection{PINCH-1}

Particularly interesting cysteine-histidine-rich protein-1 (PINCH-1) is a cytoplasmic component of cellextracellular matrix adhesions required for protection of multiple types of cancer cells from apoptosis. Although PINCH-1 is not a transcription factor, its knockdown leads to a 10-fold increase in Bim $_{\mathrm{EL}} \mathrm{mRNA}$ levels, suggesting for a role of PINCH-1 in suppressing Bim transcription [210]. Depletion of Bim blocked apoptosis induced by the loss of PINCH-1 [210]. Besides affecting Bim transcription, 
PINCH-1 promotes activating phosphorylation of Src family kinases and ERK1/2 that leads to Ser69 phosphorylation of Bim resulting in enhanced degradation [210].

\subsubsection{Pokemon/Lrf}

The erythroid myeloid ontogenic factor Pokemon (also known as Lrf/Zbtb7a) is a member of the POK (POZ and Krüppel) family of transcriptional repressors that is a master regulator of $\mathrm{B}$ and $\mathrm{T}$ cell lymphoid fates and erythroid development and maturation [211, 212]. Pokemon was found to directly repress Bim transcription through a tandem-binding site in the bim proximal promoter region [212]. As Pokemon is a downstream target of GATA-1, the expression of GATA-1 indirectly promotes erythroid cell survival [212]. Thus, Pokemon facilitates erythroid differentiation and survival through prevention of Bim expression. Maeda et al. [212] further showed that Bim deficiency rescues the anemia observed in Lrf/Pokemon KO mice.

\subsubsection{SP-1}

SP-1 (Specificity Protein 1) is often overexpressed in cancer and may be a negative prognostic factor for the overall survival $[213,214]$. The bim promoter contains six GGGCGG motifs that are recognized by the transcription factor SP-1 [17]. Inhibition of SP-1 by the curcumin analogue dibenzylideneacetone, led to increased expression of Bim and apoptosis in mucoepidermoid carcinomas [215], suggesting that SP-1 acts as a suppressor of Bim expression. Also mithramycin A, another SP-1 inhibitor induced Bim expression and apoptosis [215]. SP-1 may positively regulate the antiapoptotic Mcl-1 expression [216], such that inhibition of SP-1 has dual pro-apoptotic effects.

\subsubsection{Brd4}

In malignant peripheral nerve sheath tumors (MPNSTs), Bim expression is repressed by the chromatin regulator Brd4, which is a member of the bromodomain and extra-terminal (BET) protein family [217]. Brd4 contains two bromodomains in tandem that permit

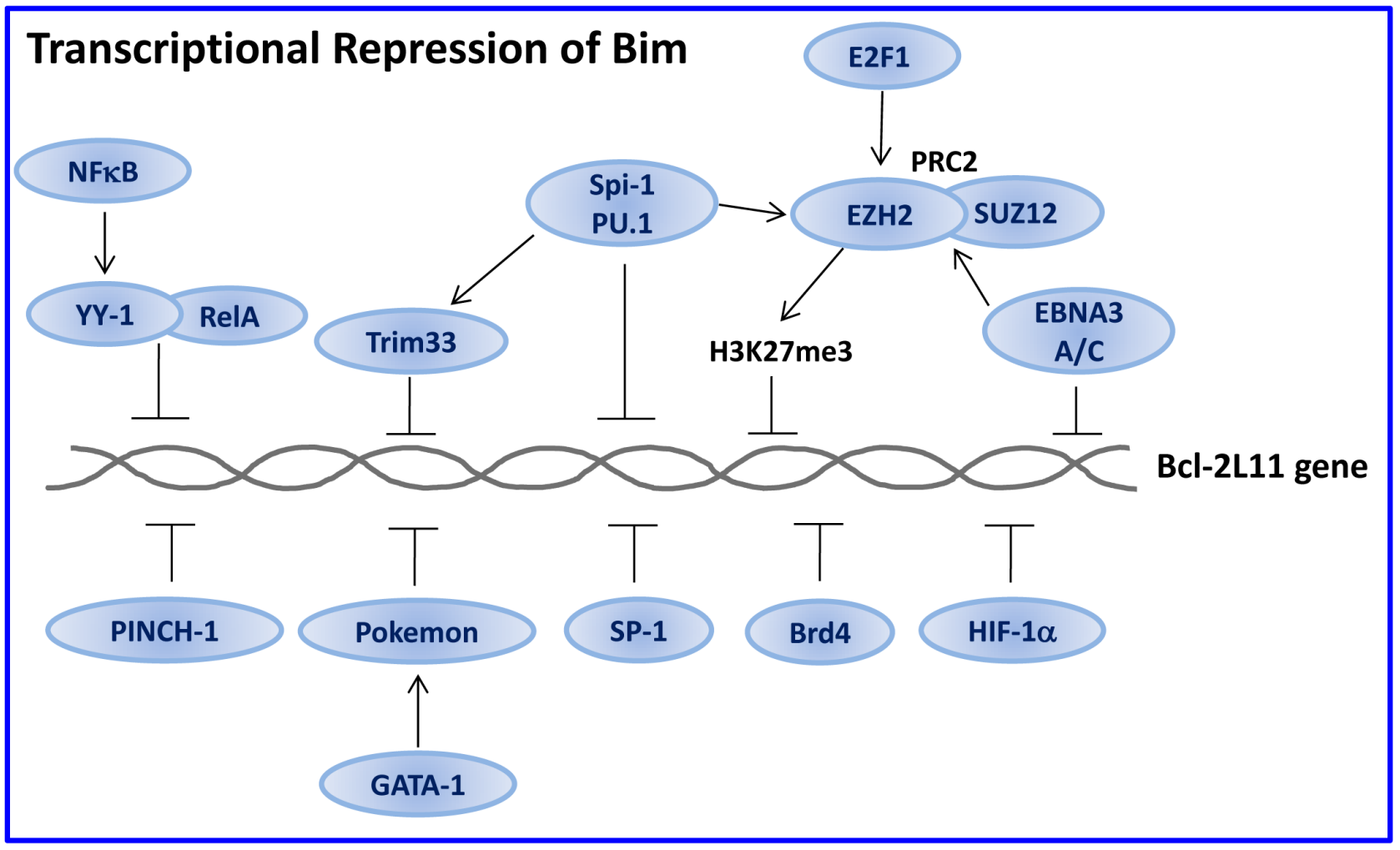

Figure 5: Transcriptional repression of Bim. Several transcription factors prevent Bim transcription. Some of them, e.g., NFkB and E2F-1, play a dual role by regulating both Bim transactivation (Figure 4) and transrepression. The RelA component of NFkB acts as a transactivator in the absence of YY-1, while becomes a transrepressor in its presence. E2F1 may directly or indirectly transactivate Bim by inducing c-Myc and B-Myb expression (Figure 4), but, in addition, it induces the polycomb histone methyl transferase EZH2 that promotes trimethylation of Histone 3 on Lys27 (H3K27me3), leading to the repression of Bim expression. Spi-1/PU.1 and Trim33 cooperate with EZH2 to repress Bim transcription. Furthermore, E2F1 directly or through c-Myc induces miR106 25 that prevents Bim translation (Figure 6). The multiple effects of E2F1 may ensure fine tuning of Bim expression. HIF-1 $\alpha$ that is, among others, induced by hypoxic conditions within the tumor microenvironment, prevents Bim expression, thus providing a growth advantage to the tumor cells. Description of the other repressors can be found in Section 3.1.2. 
recognition and binding to acetylated histones, and subsequent recruitment of co-factors (including $\mathrm{pTEFb}$ ) for RNA polymerase II-dependent elongation, resulting in the upregulation of $\mathrm{c}-\mathrm{Myc}$ and $\mathrm{Bcl}-2$. Inhibition of Brd4 with either of the small molecule inhibitors JQ1, I-BET 151 or CPI203, induced apoptosis of these malignant cells through induction of Bim along with downregulation of Bcl-2 and Cyclin D1 [217]. Cotreatment with the Brd4 inhibitor JQ1 and the HDAC inhibitor panobinostat synergistically induced apoptosis of human acute myelogenous leukemia (AML) blast progenitor cells (BPC) through attenuation of c-Myc and Bcl-2 expression, while increasing p21 and Bim expression [218]. Similarly, the Brd4 inhibitor I-BET151 induced Bim-dependent apoptosis in melanoma cells that was not affected by the B-Raf and N-Ras mutational status [219]. JQ1 also had a synergistic effect on Flt3 tyrosine kinase inhibitor (ponatinib)-induced apoptosis of $\mathrm{CD}^{+} 4^{+}$human $\mathrm{AML}$ BPC cells, which led to increased upregulation of Bim [220]. JQ1 downregulated c-Myc and Birc5 expression in primary paedriatric B-precursor acute lymphoblastic leukemia (B-precursor ALL) and sensitized the malignant cells to dexamethasone-induced apoptosis [221]. The therapeutic effect of JQ1 was dependent on basal high c-Myc expression [221].

\subsubsection{HIF-1 $\alpha$}

Sympathetic neurons exposed to low $\mathrm{O}_{2}$ tension upregulate hypoxia-inducible factor- $1 \alpha$ (HIF-1 $\alpha)$ that protects against NGF-deprivation-induced apoptosis through suppression of Bim expression [222]. The Tax protein of human T-lymphotropic virus type 1 (HTLV-1) downregulates Bim expression at the transcriptional level through induction of HIF-1 $\alpha$ [223]. Knockdown of HIF$1 \alpha$ or chemical inhibition of its transactivation activity resulted in increased Bim expression and sensitization of HTLV-1-infected leukemic T cells to CD95/TRAIL- and anticancer drug-induced apoptosis [223]. HIF-1 $\alpha$ may also reduce Bim protein expression through ERK-dependent phosphorylation and degradation [224].

\subsubsection{EBNA3A and EBNA3C}

Epstein-Barr virus (EBV) contributes to the development of various human cancers including the endemic form of Burkitt's lymphoma. The nuclear factors EBNA3A and EBNA3C repress Bim transcription that leads to the establishment of lymphoblastoid cell lines from primary B cells [225]. EBNA3C binds near the transcription start site and recruits $\mathrm{PRC} 2$ core subunits that are required for Bim repression [226]. McClellan et al. [227] identified EBNA binding sites between +87 and +921 , which are downstream to the transcriptional start site described by Paschos et al. [226].

\subsubsection{Alternative splicing}

As described in Section 1.3, the Bim transcripts undergo extensive alternative splicing, giving rise to a variety of Bim isoforms with different intrinsic toxicities and modes of regulation $[3,25,28]$. Splicing of exon 3 and splicing of exon 4 usually occur in a mutual exclusive manner, and due to the presence of a stop codon and a polyadenylated signal within exon 3 , its inclusion leads to a pre-mature Bim protein that lacks the $\mathrm{BH} 3$ domain required for apoptosis [30, 31, 228]. Intron 2 deletion polymorphism led to preferential splicing of exon 3 over exon 4, thus excluding the BH3 encoded by exon 4 [31]. Bim $\gamma$ formed by the splicing of exon 3 had a very short half-life of less than an hour [31].

The splicing factor oncoprotein SRSF1 (also known as SFSR1 or SF2/ASF) promoted alternative splicing of Bim to produce isoforms that lack pro-apoptotic functions [229]. Two new isoforms termed Bim $\gamma 1$ and Bim $\gamma 2$ were generated due to the inclusion of an alternative 3 , exon and exclusion of exons E4 and E5, thus lacking the $\mathrm{BH} 3$ domain [229]. The expression of the isoforms $\mathrm{Bim}_{\mathrm{EL}}, \mathrm{Bim}_{\mathrm{L}}$ and $\mathrm{Bim}_{\mathrm{S}}$ was concomitantly reduced [229]. SRSF1 is frequently upregulated in breast cancers and its overexpression leads to the formation of larger acinar cells, due to increased proliferation and delayed apoptosis during acinar morphogenesis [229]. The alternative splicing of Bim is important for the reduced apoptosis of acinar cells [229]. High SRSF1 expression occurs frequently in tumors overexpressing c-Myc, and these tumors are of higher histological grade that those with low c-Myc and SRSF1 expression [229]. Further studies showed that SRSF1 is a target gene of c-Myc [230]. Loss of SRSF1 induces G2 cell cycle arrest and apoptosis [231]. Knockout of the serine/arginine-rich (SR)-related protein Pinin (Pnn) leads to early embryonic lethality, and its knockdown in breast cancer cells leads to apoptosis due to reduced expression of SRSF1 and increased expression of Bim [232].

The human Krüppel-like zinc finger protein Glisimilar (GLIS) 3 whose mutations lead to neonatal diabetes, seem to indirectly affect Bim splicing [233]. Knockdown of GLIS3 leads to preferential expression of $\mathrm{Bim}_{\mathrm{S}}$ and $\beta$-cell apoptosis that was further aggravated by cytokines [233]. Simultaneous knockdown of Bim abrogated the pro-apoptotic effect of GLIS3 [233]. One mechanism by which GLIS3 prevents Bim $_{\mathrm{S}}$ isoform formation is through induction of the splicing factor SRp55 (encoded by the gene splicing factor arginine/ serine (RS)-rich 6 (SFRS6)) [233]. Overexpression of GLIS3 in $\beta$-cells antagonized cytokine-induced apoptosis [233] and SRp55 expression was modified in human islets following cytokine treatment [234]. The observation by Nogueira et al. [233] showing that downregulation of SRp55 in $\beta$-cells leads to increased expression of $\mathrm{Bim}_{\mathrm{S}}$ and apoptosis, opposes the report of Jiang et al. [235] showing 
that SRp55 appears necessary for the preferential increase in $\operatorname{Bim}_{\mathrm{S}}$ splicing observed after treating B-Raf ${ }^{\mathrm{V} 600 \mathrm{E}}$ mutant human melanoma cells with the B-Raf ${ }^{V 600 E}$ inhibitor PLX4720. The apparent discrepancy could be due to cell-specific effects. Also, Hara et al. [236] observed that SRp55/SFRS6 leads to preferential upregulation of the $\operatorname{Bim}_{\mathrm{S}}$ isoform following treatment of human neuroblastoma cells with $\mathrm{Zn}^{2+}$, that induces apoptosis of these cells. They identified a SFRS6 binding site in the intronic region adjacent to exon 4 [236].

Juan et al. [237] studied the 2,903-bp deletion polymorphism within Bim intron 2 that biased towards exon 3, leading to impaired Bim-dependent apoptosis. They observed that this region has many cis-acting elements that repress exon 3 inclusion [237]. A 23-nt intronic splicing silencer at the 3'-end of the deletion was found to be important for the exon 3 exclusion [237]. They demonstrated that Polypyrimidine tract binding protein 1 (PTBP1) and Heterogeneous nuclear ribonucleoprotein C (hnRNP C) repress exon 3 inclusion, and downregulation of PTBP1 inhibits Bim-mediated imatinib-induced apoptosis [237].

The catalytic subunit Brm (Brahma) of the SWI/SNF related, matrix associated, subfamily a, member 2 (also known as mating-type switch/sucrose nonfermenting) complex involved in chromatin remodeling on promoters was shown to associate with several components of the spliceosome and with Sam68, an ERK-activated enhancer of variant exon inclusion [238]. Brm favors the inclusion of variant exons in the mRNA of Bim, in addition to increasing the Bim expression level [238]. The BH3containing exon 4 (that they coined exon 5) was included between exon 2 and 5 (that they coined exon 7), giving rise to the $\mathrm{Bim}_{\mathrm{L}}$ and $\mathrm{Bim}_{\mathrm{EL}}$ isoforms [238]. The Brm gene codes for an ATPase catalytic subunit that shifts histones and opens the chromatin and is considered to be a tumor suppressor gene [239].

As Bim $_{\mathrm{S}}$ can directly bind and activate Bax [9, $23,24]$, and is not sequestrated to the cytoskeleton [22] or negatively regulated by the MEK/ERK pathway [17, 25], makes its preferential upregulation a significant contribution to apoptosis. The more potent pro-apoptotic function of Bim $_{\mathrm{S}}$ may explain why this isoform is barely expressed under basal conditions, in contrast to the $\mathrm{Bim}_{\mathrm{EL}}$ isoform that is abundantly expressed $[14,17,20,25,63$, 64].

\subsubsection{Epigenetic Regulation of the bim and foxO3 Promoters}

Bim expression is also epigenetically regulated. Histone deacetylase inhibitors (HDACi) induce Bim expression in various tumors, including multiple myeloma, melanoma, pancreatic adenocarcinoma, Burkitt's lymphoma and CML [240-245]. Silencing of the bim promoter by hypermethylation has been shown in B cell lymphomas [246]. In human B cells infected with Epstein-Barr virus (EBV), latency-associated virus gene products inhibit expression of Bim and enhance cell survival. Besides the involvement of the EBV nuclear proteins EBNA3A and EBNA3C in repressing Bim transcription, latent EBV initiates a chain of events that leads to epigenetic repression of Bim in infected B cells and their progeny [243]. There was a significant methylation of $\mathrm{CpG}$ dinucleotides within the large $\mathrm{CpG}$ island located at the 5' end of bim in EBV-positive, but not in EBV-negative B cells [243]. Paschos et al. [243] also observed that EBV-mediated repression of Bim was associated with reduced acetylation of histones H3 and H4. IGF-1 treatment of multiple myeloma cells led to reduced histone $\mathrm{H} 3$ tail Lys9 (H3K9) acetylation, and increased $\mathrm{H} 3 \mathrm{~K} 9$ dimethylation, which contributed to the silencing of the bim and foxO3 genes [247]. Patients with Burkitt's lymphoma with hypermethylated bim showed lower complete remission rate and shorter overall survival than those with Bim-expressing lymphomas [244].

Downregulation of Bim expression was observed in $36 \%$ of Bcr-Abl-positive chronic myeloid leukemia patients that could explain their sub-optimal response to imatinib [248]. The reduced expression of Bim was due to promoter hypermethylation that could be prevented by 5-aza-2'-deoxycytidine treatment [248]. The MAPK p38-mediated phosphorylation of the histone H2AX at Ser139 was found to be necessary for the imatinib-induced Bim expression in chronic myeloid leukemia cells [249]. $\mathrm{H} 2 \mathrm{AX}$ regulates DNA repair after being phosphorylated at Ser139 by ATM [250], but is involved in apoptosis of cancer cells that depends on the phosphorylation of the same serine residue by Mst1 (mammalian STE20-like kinase 1) [251] or JNK [252]. Knockout of H2AX blocked apoptosis [252].

\subsection{Post-Transcriptional Regulation}

\subsubsection{Regulation of Bim mRNA through the 3'-Untranslated Region (3'-UTR)}

Translation of Bim mRNA can be regulated through the 3'-untranslated region (3'-UTR) [253, 254]. This region binds microRNAs and RNA-binding proteins (RBPs) that regulate mRNA stability and/or translation [255]. Cytokines negatively regulate the steady-state levels of Bim through heat-shock cognate protein 70 (Hsc70), which binds to AU-rich elements (AREs) in the 3'-UTR [253]. The RNA binding potential of $\mathrm{Hsc} 70$ is regulated by the co-chaperones Bag-4 (SODD), CHIP, Hip, and Hsp40. Cytokines regulate the expression or function of these cochaperones by activating Ras pathways. Thus, exposure of cells to cytokines ultimately leads to destabilization of Bim mRNA and promotion of cell survival [253].

Heat shock protein 27 (Hsp27) prevents oxidative 
stress-induced cell death in cerebellar granule neurons by binding to the 3'-UTR of the Bim mRNA, thereby preventing translation of Bim protein [256]. Oxidative stress induced by hydrogen peroxide led to Hsp27 depletion and increased Bim expression, resulting in subsequent neuronal death [256]. Of note, HMG-CoA reductase inhibitors (statins) increased Hsp27 expression [257] and prevented oxidative stress-induced apoptosis of endothelial progenitor cells by inhibiting FoxO4-mediated upregulation of Bim [258].

\subsection{2. microRNAs Regulating Bim Expression}

Bim expression is negatively regulated by a variety of microRNAs. The importance of microRNAs in Bim regulation is demonstrated in embryonic stem cells deficient in the Argonaute (Ago) 1-4 proteins that are the core effectors of the microRNA pathway [259]. These embryonic stem cells are defective in microRNA silencing, show upregulated expression of the three isoforms of Bim $\left(\mathrm{Bim}_{\mathrm{EL}}, \mathrm{Bim}_{\mathrm{L}}\right.$ and $\left.\mathrm{Bim}_{\mathrm{S}}\right)$ and undergo apoptosis [259]. The upregulation of Bim was sufficient to induce apoptosis that could be prevented by simultaneous expression of activated Akt [259]. Reintroduction of any single Ago into Ago-deficient cells was able to rescue the endogenous miRNA silencing defect and apoptosis [259]. Similarly, Dicer-deficient pro-B cells showed enhanced upregulation of Bim and developmental block at the pro- to pre-B cell transition with excessive apoptosis at the pre-B cell stage [260]. B cell development could be partially rescued by ablation of Bim or transgenic overexpression of Bcl-2 [260].

\subsubsection{1. $\mathrm{miR}-9$ and $\mathrm{miR}-181 \mathrm{a}$}

miR-9 and miR-181a were downregulated in the synovial fluid cells after tibial plateau fractures, leading to increased expression of the Cbl E3 ubiquitin ligase

\section{Translational Regulation of Bim Expression}
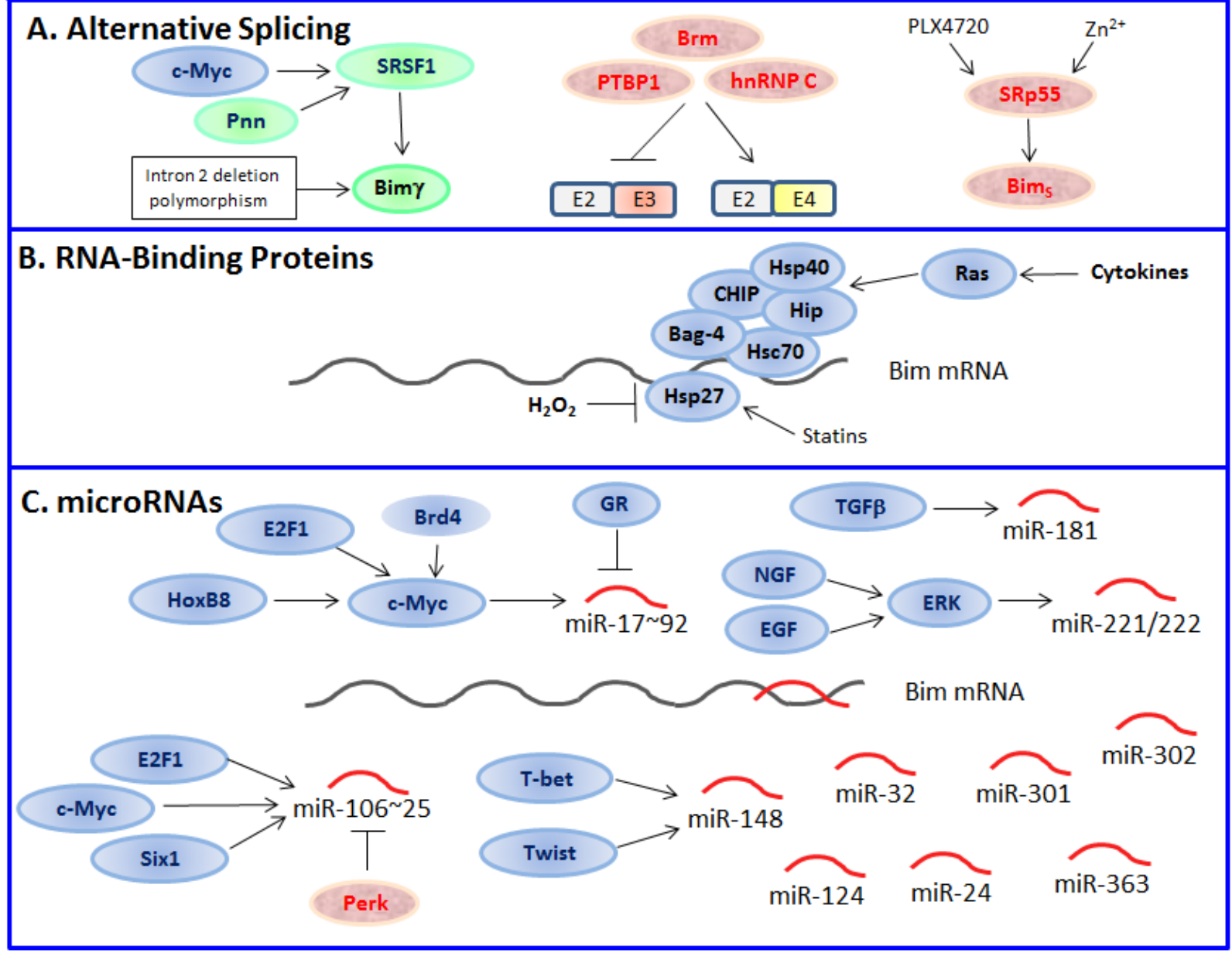

Figure 6: Translational regulation of Bim. A. Various Bim isoforms can be formed by alternative splicing, where upregulation of $\mathrm{Bim}_{\mathrm{S}}$ is especially efficient in inducing apoptosis, while formation of BH3-deficient Bim $\gamma$ lacks pro-apoptotic activity. The splicing factor

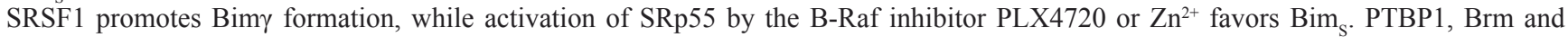
hnRNP2 favor the inclusion of the BH3-containing E4 exon over the E3 exon. B. Various RNA-binding proteins such as Hsp27 and Hsc70 prevent Bim translation. C. A range of microRNAs can target Bim, among them miR-17 92, miR-106 25, miR-181, miR-148 and miR$221 / 222$ are considered as oncomiRs that can promote tumor progression. 
involved in bone formation and homeostasis regulation [261]. The elevated levels of Cbl led to increased Bim ubiquitination and degradation, resulting in enhanced osteoclast survival and activation [261]. Also, TGF $\beta$ upregulates miR-181 expression to promote breast cancer metastasis [262]. Inactivation of miR-181a elevated the expression of Bim, which sensitized metastatic cells to anoikis [262]. This research group further showed that miR-181a expression was essential in driving pulmonary micrometastatic outgrowth and enhancing the lethality of late-stage mammary tumors in mice [262]. miR-181a expression was upregulated in metastatic breast tumors, particularly triple-negative breast cancers, and was highly predictive for decreased overall survival in human breast cancer patients [262].

\subsubsection{The miR-17 92 and miR-106b 25 clusters}

The 3'-UTR of Bim mRNA contains nine potential binding sites for miR-17 92 family members with at least one binding site for each of the three distinct microRNA seeds related to the miR-17 92 cluster [260]. miR-92, and to a lesser extent miR-19, targets the Bim 3'-UTR in reporter assays in HeLa cells [263]. Genetically engineered mice with higher expression of miR-17 92 in lymphocytes developed lymphoproliferative disease and autoimmunity and died prematurely [263]. Lymphocytes from these mice showed more proliferation and less activation-induced cell death [263]. In contrast, mice deficient for miR-17 92 die shortly after birth with lung hypoplasia and a ventricular septal defect as a result of increased Bim expression [264]. miR-17 and the miR17 92 cluster have also been shown to cause resistance of pediatric acute lymphoblastic leukemia to glucocorticoidinduced apoptosis through prevention of Bim expression $[265,266]$. Dexamethasone treatment led to reduced expression of the miR-17 92 cluster, with concomitant upregulation of Bim and apoptotic sensitization [265, 266]. miR-92a promotes glioma cell survival through repression of Bim [267]. miR-20, miR-92 and miR-302 are important for epiblast stem cell survival through repression of Bim [268]. Bim knockout rescued the cell death phenotype in epiblasts of Dicer/- embryos [268].

miR-25 of the miR-106b 25 cluster prevents Bim expression in human ovarian cancer cells, promoting their survival [269]. miR-25 also suppresses Bim expression in gastric cancer [175]. The miR-106b 25 polycistron is activated by genomic amplification and is potentially involved in esophageal neoplastic progression [270]. Repression of the miR-106b 25 cluster by PKR-like endoplasmic reticulum kinase (Perk) was required for ER stress-induced apoptosis that is mediated by Bim [271]. The HDAC inhibitor trichostatin suppressed miR106b 93 25 expression through downregulation of c-Myc, thereby inducing apoptosis in human endometrial cancer cells [272]. Besides c-Myc, E2F1 positively regulates the expression of the intronic microRNAs 106b 93 25 [175]. Of note, myc is a direct target gene of E2F1 [273]. Overexpression of the miR-106b 25 cluster in gastric cancer prevented TGF $\beta$-induced Bim expression and apoptosis [175]. Further studies showed that miR-25, but not miR-106b or miR-93, was responsible for the downregulation of Bim [175]. microRNAs 106b and 93, however, prevent E2F1 expression, forming a negative feedback loop [175].

\subsubsection{3. miR-148a}

In repeatedly activated T helper type 1 (Th1) cells, Bim expression is downregulated posttranscriptionally by miR-148a [274]. The expression of miR-148a is induced by the transcription factors T-bet and Twist1, which promotes persistence of antigen-specific Th1 cells in long-lasting, chronic immune reactions [274]. Inhibition of miR-148 increased Bim expression and apoptosis of Th1 cells [274]. miR-148a is expressed at higher levels in $\mathrm{T}$ cells from rheumatoid arthritis patients than healthy donors [274]. miR-148a is also involved in the survival of glioblastoma cells through downregulation of Bim [275]. Higher levels of miR-148a expression were a risk indicator for glioblastoma patient survival [275].

\subsubsection{Other microRNAs regulating Bim expression}

Upregulation of miR-32 by 1,25-dihydroxyvitamin D3 in human myeloid leukemia cells leads to targeting of Bim and prevention of arabinocytosine (AraC)-induced apoptosis [276]. miR-32 was also found to target Bim in prostate cancer [277]. miR-301a promotes pancreatic cancer cell proliferation by inhibiting Bim expression [278], while miR-363 supports human glioblastoma stem cell survival for the same reason [279].

NGF increases the expression of miR-221/222 in PC12 cells through the ERK pathway. These microRNAs bind to the 3'-UTR of Bim mRNA, thereby preventing Bim translation [254]. miR-124 that is abundantly expressed in midbrain dopaminergic neurons, was downregulated in a mouse model of Parkinson's disease, accompanied by an increase in Bim expression and death of the neurons [280].

miR-24 was found to suppress cardiomyocyte apoptosis in part through repressing Bim [281]. Forced overexpression of miR-24 in a mouse myocardial infarction model inhibited cardiomyocyte apoptosis, attenuated infarct size and reduced cardiac dysfunction [281].

\subsection{2. microRNAs affecting Bim-regulating factors}

microRNAs that downregulate FoxO3 expression, indirectly affect Bim expression [13]. These include miR-1, miR-27a, miR-96, miR-155, miR-182 and miR221/222 [13]. For instance, aberrant microRNA-182 expression was associated with glucocorticoid resistance in lymphoblastic malignancies [282]. microRNA-182 reduced FoxO3a expression with consequent reduced $\mathrm{Bim}$ expression [282]. miR-155 facilitates lymphoproliferation 
induced by a mutant version of the adaptor protein Linker for Activation of T cells (LAT) via inhibition of FoxO3dependent Bim expression [283]. miR-155 reduces the expression of the inositol phosphatase Src homology-2 domain-containing inositol 5-phosphatase 1 (SHIP1) [284], thereby promoting Akt-mediated inactivation of FoxO3 [283].

Reduced expression of miR-101 was associated with overexpression of the Bim transcriptional repressor EZH2 in NSCLC [285]. Overexpression of miR-101 sensitized NSCLC cells to paclitaxel-induced apoptosis through inducing Bim expression [285].

microRNAs targeting Phosphatase and tensin homolog deleted on chromosome 10 (PTEN) enhance Akt signaling, resulting in inhibition of apoptosis and Bim expression. These include miR-17 92, miR-106 25, miR21, miR-26a, miR-29b, miR-212, miR-216a, miR-217 and miR-221/222 [13]. Of note, miR-17 92 targets both PTEN and Bim [263], making it a potential therapeutic target for increasing the response of anti-cancer drugs. In lymphoid malignancies, glucocorticoids repress miR-17 92, leading to increased Bim expression and drug response [266]. miR-221/222, which is frequently upregulated in cancer [286], targets Bim, PTEN and FoxO3, thus fortifying apoptotic resistance.

microRNAs that target the transcription of antiapoptotic proteins, also indirectly affect the pro-apoptotic function of Bim. Overexpression of Bcl-2 is common in $\mathrm{B}$ cell chronic lymphocytic leukemia (B-CLL) due to loss or downregulation of the human chromosome 13q14 locus, which harbors the miR-15a and miR-16-1 cluster [287]. miR-34a, miR-125b and miR-181c may also target Bcl-2 $[13,288]$. Mcl-1 is targeted by miR-29a [289], miR-218 [290], miR-101 [291] and miR-193b [292]. MicroRNAs regulating Fbw7, such as miR-223, miR-27a, miR-25 and miR-129-5p [293] might also indirectly affect Bimdependent apoptosis through Fbw7-mediated regulation of c-Myc, Mcl-1, Notch1 and c-Jun [293, 294].

\subsection{Post-Translational Regulation}

\subsubsection{Regulation of Bim Activity by Protein Kinases}

The phosphorylation status of Bim controls its proapoptotic activity and stability [7, 25, 295]. Especially, the MAP kinases are involved in the regulation of the proapoptotic activity of Bim [25]. In general, phosphorylation of Bim by ERK1/2 leads to the degradation of Bim through the ubiquitin-proteasome pathway, while its phosphorylation by JNK or p38 increases its activity. The Akt/mTOR pathway indirectly affects Bim through phosphorylation and inactivation of the FoxO transcription factors and upregulation of the anti-apoptotic Mcl-1 protein. Thus, receptor signaling or oncogenic addictions activating ERK, Akt and/or mTOR signaling pathways confer apoptotic resistance through reducing Bim expression or antagonizing its activity.

Mutation studies showed that mutation of the phosphorylation site Thr112 in mouse Bim caused decreased binding of Bim to the anti-apoptotic protein Bcl2 and increased cell survival [295]. However, mutation of the phosphorylation sites Ser55, Ser65, and Ser73 in mouse Bim caused increased apoptosis because of reduced proteasomal degradation of Bim [295]. The amino acid positions differ somewhat between human and mouse Bim (Figure 7), so we have adapted in the text the numbers described in the cited references.

\subsubsection{ERK1/2}

Phosphorylation of BIM $_{\mathrm{EL}}$ by ERK1/2 at Ser69 in human or at Ser65 in mouse leads to K48-linked ubiquitindependent $26 \mathrm{~S}$ proteasome-mediated degradation of

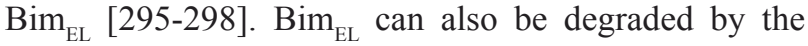
$20 \mathrm{~S}$ proteasome in the absence of poly-ubiquitination, as shown by mutating the only two lysine residues Lys3 and Lys 108 in rat Bim [298]. This might be related to the fact that Bim $_{E L}$ is an intrinsically unstructured protein [299]. The degradation in absence of poly-ubiquitination is prevented when Bim $_{\mathrm{EL}}$ is bound to Mcl-1 [298]. Since exon E2B encodes the ERK1/2-docking domain and ERK1/2 phosphorylation sites, only Bim $_{E L}$, but not Bim $_{L}$ or $\mathrm{Bim}_{\mathrm{S}}$, is subject to phosphorylation by the MEK/ERK pathway [296, 300]. In addition, ERK phosphorylation of mouse Bim at Ser65 prevents its binding to Bax [296, 300], and leads to its dissociation from Mcl-1 and Bcl$x L[301,302]$. Binding of Bim to Mcl-1 stabilizes the latter [303]. This is in contrast to the binding of Noxa to Mcl-1 that promotes Mcl-1 degradation [303]. Thus, Bim dissociation from Mcl-1 leads to destabilization of both the pro-apoptotic Bim and the anti-apoptotic Mcl-1, the net effect determining the cell fate.

The hematopoietic survival factor Interleukin-3 (IL3 ) induces ERK-mediated phosphorylation of mouse Bim on three serine residues (Ser55, Ser65 and Ser100) that prevents Bim from interacting with Bax [7]. Following IL-3 withdrawal, only the non-phosphorylated form of Bim interacts with Bax [7]. Survival is often promoted in tyrosine kinase-driven cancers such as CML and EGFR NSLCL, through repression of Bim transcription and through targeting the Bim protein for proteasomal degradation following Mitogen-activated protein kinase 1 (MAPK1/ERK2)-dependent phosphorylation [304-308]. Targeting EGFR using kinase inhibitors (e.g., erlotinib and gefitinib) led to suppression of PI3K/mTORC and MEK/ERK signaling, followed by an increase in Bim expression [307, 309, 310] and a decrease in Mcl-1 [310]. NGF induces MEK/MAPK-mediated phosphorylation of rat Bim $_{\mathrm{EL}}$ at Ser109 and Thr110 in sympathetic neurons [311].

Dehan et al. [312] observed that ERK1/2 co-operates with ribosomal S6 kinase (RSK) to phosphorylate Bim ${ }_{\mathrm{EL}}$, allowing binding of the $\mathrm{F}$-box proteins $\beta$-TrCP1/2, which 
promotes BIM $\mathrm{EL}_{\mathrm{EL}}$ poly-ubiquitination. Silencing of either $\beta$-TrCP or RSK1/2 resulted in $\mathrm{Bim}_{\mathrm{EL}}$-mediated apoptosis of both gefitinib-sensitive and gefitinib-insensitive NSCLC cells [312]. Phosphorylation of Ser69 of human Bim ${ }_{E L}$ by ERK1/2 promoted cytokine-induced phosphorylation on Ser93, Ser94 and Ser98, that was required for the binding of Bim $_{E L}$ with $\beta$-TrCP1 [312]. RSK1 and RSK2 were responsible for the phosphorylation on Ser93/94/98 [312].

Elevated expression of urokinase plasminogen activator (UPA) in EGFR-positive glioblastoma cells leads to apoptosis resistance to EGFR tyrosine kinase inhibitors through ERK1/2-dependent repression of Bim expression [313]. Tyrosine kinase inhibitor-resistant glioblastomas can be resensitized to EGFR tyrosine kinase inhibitors by pharmacologic inhibition of MEK or a $\mathrm{BH} 3$ mimetic drug to replace Bim function [313].

ERK1/2 inhibition leads to Bim $_{\mathrm{EL}}$ stabilization and increased tumor cell death [314, 315]. Similarly, MEK inhibitors could potentiate dexamethasone lethality of acute lymphoblastic leukemia cells through upregulating Bim expression [316]. Bim accumulated by this treatment interacted with Bcl-xL and $\mathrm{Mcl}-1$, resulting in the release of Bak [316]. Combined treatment of inhibitors of the PI3K/Akt and MEK/ERK1/2 pathways led to Bimdependent leukemia cell death [317]. The pineal gland hormone melatonin may induce apoptosis of malignant cells by inhibiting Bim degradation by the proteasome, besides inducing SP-1-, FoxO3a-, and E2F1-dependent transcriptional activation of Bim [318-320].

\subsubsection{JNK}

In contrast to ERK phosphorylation that usually leads to Bim degradation, JNK phosphorylation of Bim that occurs, for instance, after trophic factor deprivation,

\section{Post-Translational Modifications of Bim}

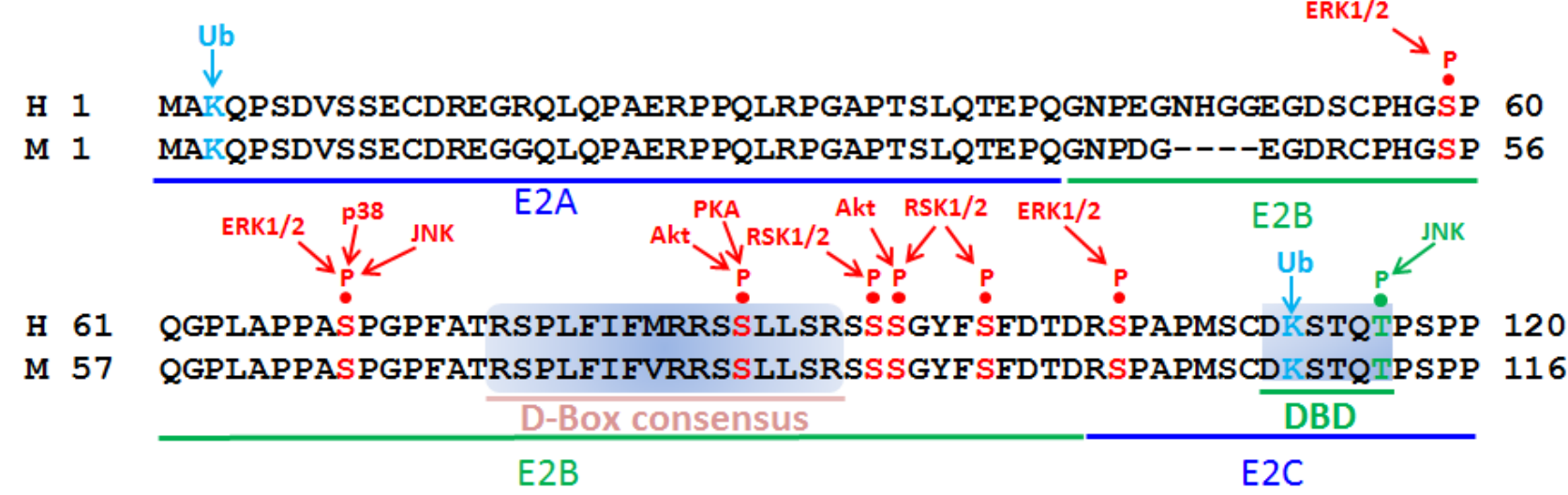

H 121 CQAFNHYLSAMASMRQA--EPADMRPEIWIAQELRRIGDEFNAYYARRVFLNNYQAAEDH 178 M 117 CQAFNHYLSAMASIRQSQEEPEDLRPEIRIAQELRRIGDEFNETYTRRVFANDYREAEDH 176 BH3

$\mathrm{E} 2 \mathrm{C}$

H 179 PRMVILRLLRYIVRLVWRMH 198

M 177 PQMVILQLLRFIFRLVWRRH 196

Hydrophobic region

E5

Figure 7: Post-translational modifications of Bim. The amino acid sequences of human $(\mathrm{H})$ and mouse $(\mathrm{M})$ Bim $\mathrm{EL}_{\mathrm{L}}$ have been aligned, and important post-translational modification sites of Bim highlighted. ERK1/2 phosphorylates Bim ${ }_{\mathrm{EL}}$ at three serine residues (Ser55, Ser65 and Ser100 in mouse corresponding to Ser59, Ser69 and Ser104 in human), which facilitate RSK1/2-mediated phosphorylation of Ser93/94/98 in human (corresponding to Ser89/90/94 in mouse), leading to ubiquitination at Lys3 and Lys108(M)/Lys112(H) by $\beta$-TrCP1 and proteasomal degradation. Aurora A phosphorylates the same residues as RSK1/2 during mitosis that occurs independently of previous ERK phosphorylation and leads to binding of $\mathrm{APC}^{\mathrm{Cdc} 20}$ to the D-box consensus region, resulting in Bim degradation. PP2A dephosphorylates Ser93/94/98 at the exit from mitosis, thereby stabilizing Bim $_{\mathrm{EL}}$. p38 and JNK also phosphorylate Bim ${ }_{\mathrm{EL}}$ at Ser65 (M)/Ser69 (H), but, in addition, JNK phosphorylates Thr112(M)/Thr116(H) which lies within the dynein-binding domain (DBD). The latter phosphorylation leads to dissociation of Bim from the microtubules. JNK may also phosphorylate Bim $\mathrm{L}_{\mathrm{L}}$ at Thr56 (same residue as Thr112 in Bim EL $_{\text {). }}$ p38 and JNK phosphorylation of Bim, at least in some cell types, lead to increased Bim activity. Also, Akt and PKA can phosphorylate Bim. The coding exons (E2A, E2B, E2C, E4 and E5) have been outlined, as well as the D-box consensus, DBD, BH3 domain and the hydrophobic C-terminal region. 
leads to phosphorylation of mouse Bim EL $_{\text {at }}$ Ser65 that potentiates its pro-apoptotic activity [156]. Another study showed that following transient focal cerebral ischemia, Bim $_{\mathrm{L}}$ is phosphorylated by $\mathrm{JNK}$, enhancing the interaction between $\operatorname{Bim}_{L}$ and $\operatorname{Bax}$ [321]. The Bax translocation from cytosol to mitochondria was dependent on JNK activity [321]. JNK also phosphorylates Thr112 in mouse $\operatorname{Bim}_{\mathrm{EL}}[295,322]$ that lies within the dynein binding motif (aa 107-112), leading to the dissociation of Bim $_{\mathrm{EL}}$ from the microtubules. UV exposure of 293 human embryonic kidney (HEK) cells led to JNK-mediated phosphorylation of $\operatorname{Bim}_{\mathrm{L}}$ at Thr56 [90].

The controversy that ERK promotes survival while JNK triggers apoptosis by phosphorylating the same site (Ser65 in mice/Ser69 in human) in Bim EL [25], might be explained by requirement of additional modifications of Bim that affect its activity. As described above, ERK1/2 phosphorylates Ser55 and Ser100 in addition to Ser65, making a docking site for RSK1/2, while JNK phosphorylates Thr112 in addition to Ser65. Becker and Bonni [323] elegantly showed that the prolyl isomerase Pin1 interacts with Ser65-phosphorylated Bim $_{\mathrm{EL}}$ in neurons. Pin1 is enriched at the mitochondrial membrane in neurons, where it forms a physical complex with the neuron-specific JNK scaffold protein JIP3 [323]. Activation of JNK signaling induces the dissociation of Pin1 from JIP3 and concomitantly promotes Pin1 binding to phosphorylated $\mathrm{Bim}_{\mathrm{EL}}$. The interaction of Pin1 with phosphorylated Bim $_{\mathrm{EL}}$ stabilizes $\mathrm{Bim}_{\mathrm{EL}}$ and thereby activates neuronal apoptosis [323]. It could also be a cellspecific phenomenon. Most of the studies showed JNKmediated phosphorylation of Bim $_{\mathrm{EL}}$ in neurons [156, 324], while ERK-mediated phosphorylation is mainly detected in non-neuronal cells [297, 300, 325].

In the $\mathrm{T}$ cell acute lymphoblastic leukemia (T-ALL) cell line Sup-T, JNK-mediated phosphorylation of Bim promotes proteasomal Bim degradation [326]. Pretreatment of these T-ALL cells with a specific JNK inhibitor, SP600125, increased the Bim $_{\mathrm{EL}}$ level and sensitized the cells to etoposide-induced apoptosis [326]. This may also explain why SP600125 sensitizes glucocorticoid-resistant lymphoid cells to glucocorticoidinduced apoptosis [63]. Thus, the outcome of JNK phosphorylation of Bim seems to be cell-dependent.

\subsubsection{3. p38 MAPK}

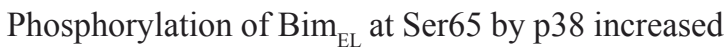
its pro-apoptotic activity [327]. Sodium arseniteinduced apoptosis in PC12 cells may be due to the direct phosphorylation of Bim $_{\mathrm{EL}}$ at Ser65 by p38 [327]. p38 may indirectly increase Bim transcription through positive regulation of FoxO3a [139], Runx2 and c-Jun [159].

\subsubsection{Akt}

Bim transcription is downregulated upon activation of Akt through Akt-mediated phosphorylation and inactivation of FoxO3a [122]. Akt phosphorylates
FoxO3a at Thr32, Ser253 and Ser315, leading to its cytoplasmic sequestration by 14-3-3 proteins, thus preventing its nuclear translocation [328]. In addition, Akt phosphorylates Mst1 on Thr387, preventing its kinase activity on FoxO3 [329]. Mst1 mediates oxidative stressinduced neuronal apoptosis by phosphorylating FoxO3 on Ser207 that leads to disruption of its association with 14-3-3 and triggers its nuclear translocation [330]. c-Abl phosphorylates Mst1 at Tyr433 in response to oxidative stress in neuronal cells, leading to its stabilization and activation of FoxO3-mediated Bim expression [331]. In contrast, the Bcr-Abl phusion protein in CML activates Akt [332] through Ubiquitin-specific-processing protease 7 (USP7)-mediated nuclear exclusion of PTEN [333] and CK2-mediated phosphorylation and inactivation of PTEN [334].

In multiple myeloma, Bim expression is downregulated by IL-6 and adhesion to fibronectin [86, 335] that might be related to Akt activation. Qi et al. [336] provided evidence that Ser87 of Bim EL $_{\text {could be }}$ phosphorylated by Akt following IL-3-stimulation of $\mathrm{Ba} /$ F3 B cells, leading to Bim $_{\mathrm{EL}}$ binding to $14-3-3$ proteins and attenuation of its pro-apoptotic function.

The oncogenic enzyme Sphingosine kinase 1 (SphK1) that phosphorylates sphingosine to sphingosine1-phosphate (S1P), is overexpressed in some types of cancer including glioma and gastric carcinoma, leading to apoptotic resistance. This kinase downregulates Bim expression through activation of the Akt pathway and inhibition of FoxO3a [337, 338]. Expression of the sphingosine-1-phosphate receptor S1P1 renders CCL39 lung fibroblasts resistant to apoptosis following growth factor withdrawal, which was associated with attenuated accumulation of Bim [339].

BMCC1 (BNIP2 and Cdc42GAP homology (BCH) motif-containing molecule at the carboxyl-terminal region 1), which is highly expressed in neuronal and epithelial tissues as well as in favorable neuroblastoma, promotes apoptosis by suppressing the PDK1/Akt pathway leading to increased FoxO3a-induced Bim transcription [340]. The $\mathrm{BNIP} 2$ homology region of $\mathrm{BMCC} 1$ may also interact with Bcl-2 [340].

\subsubsection{Protein Kinase A}

Treatment of T lymphoma cells with the Protein kinase A (PKA) agonist 8-CPT-cAMP led to the phosphorylation of cAMP response element-binding protein (CREB) and induction of Bim, followed by apoptosis [341]. The cytotoxic effect of 8-CPT-cAMP was dependent on PKA and Bim [342]. The PRKAR1A regulatory subunit of cyclin-dependent PKA was found to interact with Bim $_{\mathrm{EL}}$ [343]. Phosphorylation of mouse Bim $_{\mathrm{EL}}$ at Ser83 (corresponding to Ser87 in human) by PKA leads to stabilization of the Bim protein and induction of apoptosis [343]. Of note, Ser83 resides within the D-box consensus sequence recognized by the Anaphase 
promoting complex $\left(\mathrm{APC}^{\mathrm{cdc} 20}\right)$ E3 ligase [344] (Figure 7, and see Section 3.3.2).

\subsubsection{Regulation of Bim Expression during Mitosis}

$\mathrm{Bim}_{\mathrm{EL}}$ was found to be regulated during mitosis by Aurora A kinase and PP2A [345]. Bim ${ }_{\mathrm{FL}}$ is phosphorylated at the $\beta-\operatorname{TrCP} 1$ phosphodegron Ser93/94/98 by Aurora A early in mitosis, and dephosphorylated by PP2A at the same residues after mitotic exit [345]. The Aurora A-mediated phosphorylation of $\mathrm{Bim}_{\mathrm{EL}}$ leads to its interaction with $\beta-\operatorname{TrCP} 1$, resulting in the ubiquitination and degradation of $\mathrm{Bim}_{\mathrm{EL}}$ [345]. Inhibition of either Cdk1 or Aurora A prevented the phosphorylation of Ser93/94/98, suggesting a cross-talk between these two kinases [345]. In accordance with this report, Mac Fhearraigh et al. [346] found that Bim undergoes transient phosphorylation during normal mitosis in K562 cells. The transition of K562 cells from mitosis to G1 resulted in the loss of $\mathrm{Bim}_{\mathrm{EL}}$ and $\mathrm{Bim}_{\mathrm{L}}$ phosphorylation. These authors identified the $\mathrm{Cdk} 1 / \mathrm{Cyclin}$ B1 complex to be involved in Bim phosphorylation [346]. Also, Gilley et al. [347] observed that Cdk1/Cyclin B1 is involved in mitotic phosphorylation of $\mathrm{Bim}_{\mathrm{EL}}$, which drives its polyubiquitination and proteasome-dependent degradation. A direct interaction between Cyclin B1 and Bim was observed in mitotic extracts [346, 347]. $\mathrm{Bim}_{\mathrm{EL}}$ and $\mathrm{Bim}_{\mathrm{L}}$ are also phosphorylated in mitosis by an MEK/ERK-dependent mechanism downstream to basic fibroblast growth factor (bFGF) signaling [348]. Phosphorylated Bim might facilitate the ordered execution of mitosis in virtue of its reduced pro-apoptotic activity [348].

Besides regulating mitotic progression, the E3 ligase $\mathrm{APC}^{\mathrm{Cdc} 20}$ interacts with $\mathrm{Bim}_{\mathrm{EL}}$ and promotes its proteasomal degradation [344]. The C-terminal WD40 repeats motif of $\mathrm{APC}^{\mathrm{Cdc} 20}$ binds to the D-box consensus region of $\mathrm{Bim}_{\mathrm{EL}}$ (amino acids 76-91 in human) [344]. $\mathrm{Bim}$ abundance is reduced during mitosis when $\mathrm{APC}^{\mathrm{Cdc} 20}$ is most active [344]. Phosphorylation of Bim by ERK/ RSK was not required for $\mathrm{APC}^{\mathrm{Cdc} 20}$ to interact with Bim [344]. Knockdown of APC ${ }^{\mathrm{Cdc} 20}$ sensitized head and neck cancer cells to apoptotic stimuli by a Bim-dependent mechanism [344]. Human adult $T$ cell leukemia cells that have acquired elevated $\mathrm{APC}^{\mathrm{Cdc} 20}$ activity due to the expression of the Tax viral oncoprotein, exhibited reduced Bim expression and resistance to anti-cancer agents [344]. Overexpression of $\mathrm{APC}^{\mathrm{Cdc} 20}$ predicts poor prognosis in primary NSLCL patients [349].

Extended mitotic arrest following treatment of cancer cells with microtubule-targeting agents led to phosphorylation of Bcl-2 and Bcl-x followed by Noxadependent Mcl-1 degradation. This enabled Bimdependent cell death [350]. Under these conditions, Mcl-1 is also degraded by the E3 ubiquitin ligase Fbw7 [107].

\section{PHYSIOLOGICAL AND PATHOPHYSIOLOGICAL ASPECTS OF BIM}

Bim is important for determining the lifespan of myeloid and lymphoid cells, as these cells are increased in numbers in mice lacking Bim [2]. Bim is also required for further homeostatic regulation in situations such as the proper termination of the innate and adaptive immune responses [47]. Alterations in Bim expression are associated with several diseases (Figure 8). Too rapid or too slow elimination of activated $\mathrm{T}$ and $\mathrm{B}$ cells as a result of too high or too low Bim expression, respectively, leads to chronic infection due to incomplete immune responses or autoimmune diseases. Bim downregulation is involved in cell transformation and reduces the sensitivity of cancerous cells to various chemotherapeutic drugs [121, 246, 304-307]. Increased Bim expression contributes to increased cardiomyocyte and neuronal cell death following ischemia [281, 351], increased $\beta$-cell death resulting in diabetes [40] and enhanced neuronal cell death in Alzheimer's disease [352]. Decreased Bim expression confers protection from viral-induced hepatitis and sepsisrelated mortality $[353,354]$. Below we will discuss these aspects in more detail.

\subsection{Regulation of Bim by Growth Factor Withdrawal}

Bim expression is induced de novo following withdrawal of survival factors (cytokines, growth factors, trophic factors) or serum from primary sympathetic neurons [125, 355], lymphocytes [2, 122, 356], hematopoietic progenitor cells [26], granulocytes [357], mast cells [358, 359], osteoclasts [360], fibroblasts [361] and multiple myeloma [247], while its expression is repressed by the PI3K/Akt or MEK/ERK signaling pathways $[117,122,359,361]$ (Figure 9). In neurons the $\mathrm{JNK} / \mathrm{c}-\mathrm{Jun}$ pathway is required for Bim mRNA expression following withdrawal of NGF $[125,355,362]$, but this pathway does not seem to be important for fibroblasts [361]. Bim induction after NGF withdrawal is reduced in sympathetic neurons in mice carrying a mutant c-Jun gene that lacks activating Ser63/Ser73 phosphorylation sites [363]. Further studies showed that induction of Bim in NGF-deprived cells requires expression and activity of Cdk4 and consequent de-repression of E2 promoter binding factor (E2F)-regulated genes including members of the Myb transcription factor family [364]. Mutation in the two Myb binding sites in the bim promoter abolished Bim induction following NGF deprivation [364]. In lymphocytes, Bim mRNA expression is promoted by the FoxO3a transcription factor, which is normally repressed by Akt/PKB-mediated phosphorylation. Consequently, inhibition of PI3K or withdrawal of survival factors is 
sufficient to induce Bim expression in these cells [122].

Addition of growth factors usually leads to downregulation of Bim [26, 311, 360]. IL-3 promotes survival of hematopoietic progenitors through downregulation of Bim [26]. The IL-3-dependent downregulation of Bim is mediated through activation of Raf/MAPK and PI3K/mTOR pathways [26]. IL-3 led to Akt activation and $\mathrm{Bim}_{\mathrm{EL}}$ phosphorylation at Ser87 in $\mathrm{Ba} / \mathrm{F} 3$ cells, leading to attenuation of its pro-apoptotic function [336]. IL-7, that is important for T cell survival, inhibits Bim activity [365]. IL-4, that is essential for the activation of mature B cells, prevents upregulation of Bim induced by BCR cross-linking [356]. Similarly, stem cell factor (SCF) promotes mast cell survival via Aktmediated inactivation of FoxO3a and MEK-regulated phosphorylation of Bim [359]. Erythropoietin induced MEK/ERK-mediated phosphorylation and degradation of Bim that contributes to the survival of erythroid cells [366]. While IL-15 mediates survival of natural killer (NK) cells by Bim downregulation and Mcl-1 upregulation [367], IL-15 leads to upregulation of Bim in T cells through a JAK/STAT5-dependent mechanism [368]. The

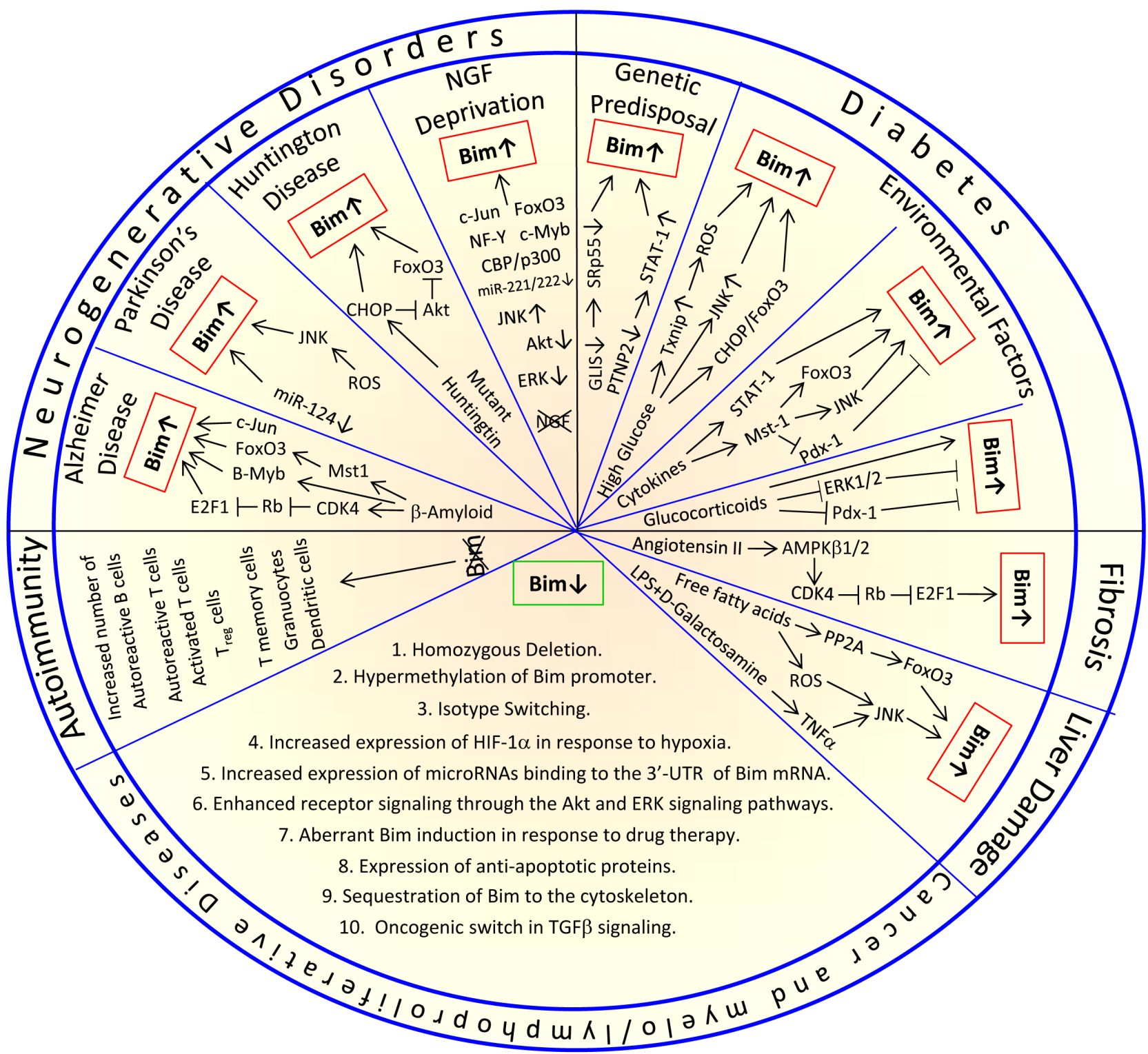

Figure 8: Abberant Bim expression in diseases. Elevated Bim expression is associated with neuronal degenerative diseases, diabetes, fibrosis and liver damage, while too low or absent Bim expression is associated with cancer (Section 4). Absent Bim expression in the immune system may lead to autoimmune diseases due to lack of elimination of autoreactive immune cells, but is, in part, compensated by an increase in regulatory $\mathrm{T}$ cells. Treatment of neurodegenerative diseases and diabetes should aim in preventing Bim expression, while treatment of cancer should aim in increasing Bim expression. Too dramatic increase in Bim during cancer treatment might thus have adverse effects on the nerve system, liver and endocrine system, that needs to be taken into account. Targeting cancer-specific pro-survival pathways would reduce the adverse effects. 
simultaneous upregulation of Mcl-1 by IL-15 counters the pro-apoptotic effects of Bim, making the T cells prone to apoptosis when the short-lived Mcl-1 is degraded [368]. IGF-1 suppresses Bim expression in multiple myeloma through several mechanisms including activation of the Akt pathway, inactivation of FoxO3, promotion of ERKinduced proteasomal degradation of $\mathrm{Bim}_{\mathrm{EL}}$ and epigenetic regulation of the bim and foxO3a promoters [247].

Glucagon-like peptide-1 (GLP-1) protected sympathetic neurons from degeneration and cell death caused by NGF withdrawal through prevention of Bim upregulation [369]. Trophoblast stem cells require fibroblast growth factor-4 (FGF4) for survival and proliferation [370]. The $\mathrm{SH} 2$ domain-containing phosphatase 2 (SHP2) was required for FGF4-evoked activation of the Src/Ras/ERK pathway that culminates in phosphorylation and destabilization of Bim [370].

\subsection{Bim in Alzheimer's disease}

\subsubsection{Role of Bim in Thrombin-Induced Apoptosis of Neurons}

The serine protease thrombin has been shown to be a potent growth factor for a variety of cell types, including vascular smooth muscle cells [371], fibroblasts [372] and some tumor cells [373], besides its central role as a coordinator of the blood coagulation cascade [374]. These cellular effects of thrombin on tissues are important for tissue repair following injury. Thrombin acts through protease-activated receptors (PARs), which are members of the G-protein-coupled-receptor superfamily [375]. Thrombin was found to prevent apoptosis of fibroblasts upon serum withdrawal through prevention of Bim upregulation [376]. This effect of thrombin was mediated

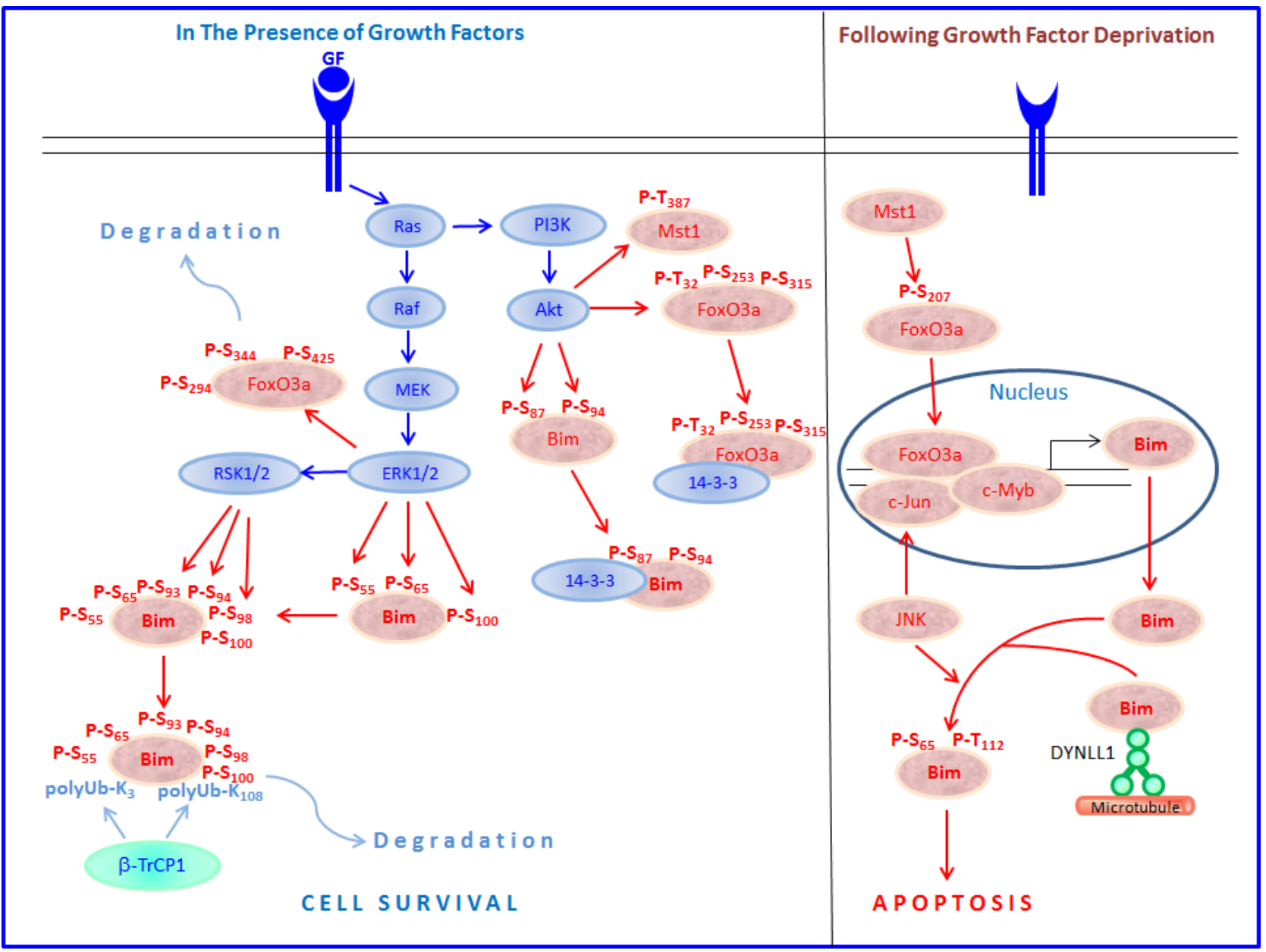

Figure 9: Growth factor-mediated cell survival versus apoptosis induction by growth factor deprivation. Growth factor receptor activation leads to activation of the Ras-Raf-MEK-ERK1/2 and PI3K-Akt pathways that co-operate in inhibiting Bim activity. ERK1/2-mediated phosphorylation of Bim $_{\mathrm{EL}}$ leads to its subsequent phosphorylation by RSK1/2 and ubiquitin-mediated proteasomal degradation. Akt-mediated phosphorylation of Bim leads to its sequestration to 14-3-3 proteins. Akt also inactivates Mst1 and FoxO3a. Following growth factor withdrawal, the lack of activation of the Ras-Raf-MEK-ERK1/2 and PI3K-Akt pathways leads to Mst1 and FoxO3a activation, resulting in the transcriptional upregulation of Bim. In addition, JNK phosphorylates Bim resulting in its activation and apoptosis induction. Inhibition of growth factor receptor signaling (e.g., by EGFR, ALK, c-Met and B-Raf ${ }^{\mathrm{V} 600 \mathrm{E}}$ inhibitors) is a major cancer-specific targeting strategy that aims to promote Bim-dependent apoptosis. 
through the activation of the Raf-MEK-ERK1/2 and PI3K pathways [376]. Thrombin also prevents apoptosis of monocytes [377], osteoblasts [378], myoblasts [379], and astrocytes [380]. However, in motor neurons, thrombin and peptide agonists of the PAR1 receptor promote apoptosis [381, 382]. Cyclin D1, Cdk4 and Bim were shown to be involved in the thrombin-induced apoptosis of cultured cerebral cortical neurons [383]. Here, Cdk4 was responsible for the upregulation of Bim [383]. These data demonstrate that thrombin-induced apoptosis proceeds via cell cycle activation. These authors suggest that cell cycle proteins might be therapeutic targets in diseases such as Alzheimer's disease where thrombin has been implicated [383]. Indeed, Cdk4 inhibitors protected neuronal cells against death induced by NGF deprivation and $\beta$-amyloid [384]. This may also explain the anti-apoptotic function of the Cdk4 inhibitor p21 [385].

The coagulation factor $\mathrm{FXa}$, which is a serine protease that plays a role during blood coagulation by converting prothrombin into active thrombin, induced apoptosis of tumor cells of epithelial origin through upregulation of Bim [386]. It is also involved in neuronal death in cerebral ischemia through activation of the JNK/c-Jun pathway [387]. In epithelial tumor cells, FXa activates ERK1/2 and p38 through PAR1 with consequent phosphorylation of the transcription factor CREB [386]. Downregulation of PAR1, Bim, ERK or p38 or the use of a dominant-negative form of CREB was sufficient to prevent FXa-mediated apoptosis of the tumor cells [386]. FXa had no effect on endothelial cells or monocytes, whereas it even enhanced fibroblast survival [386]. Of note, FXa-induced PAR1 activation in fibroblasts led to Bim downregulation [386], demonstrating a celldependent effect.

\subsubsection{Role of Bim in $\beta$-Amyloid-Induced Apoptosis}

Examination of human brains of post-mortem Alzheimer's disease patients showed that Bim is upregulated in vulnerable entorhinal cortical neurons, but not cerebellum, a region usually unaffected by the disease [352]. They further demonstrated that Bim is required for $\beta$-amyloid-induced neuronal apoptosis [352]. $\mathrm{Cdk} 4$ and its downstream effector B-Myb were required for $\beta$-amyloid-dependent Bim induction and death in cultured neurons [352]. Cdk4 inhibitors were recently shown to be neuroprotective [384]. Also, FoxO3a and AP-1 (c-Jun/c-Fos) are involved in the $\beta$-amyloid-induced upregulation of $\mathrm{Bim}[388,389]$. The activation of FoxO3a by $\beta$-amyloid is in part due to reduced Akt-mediated FoxO3a phosphorylation and increased Mst1-mediated FoxO3a phosphorylation [388]. Estrogen protects against $\beta$-amyloid peptide-induced apoptosis by upregulating Bcl-w and downregulating Bim [390].

In addition to parenchymal accumulation and neuronal degeneration in the brain of Alzheimer's disease patients, $\beta$-amyloid accumulates in the cerebrovascular wall leading to cerebral amyloid angiopathy. Amyloid- $\beta$ peptide-induced death in cerebral endothelial cells is preceded by mitochondrial dysfunction and signaling events characteristic of apoptosis. The expression of Bim, but not other BH3-only members, was selectively increased in cerebral microvessels isolated from 18-month-old APPsw (Tg2576) mice, a model of cerebral amyloid angiopathy, suggesting a pivotal role for Bim in $\beta$-amyloid-induced cerebrovascular degeneration in vivo [391]. $\beta$-Amyloid increases FoxO3a activity through activation of PP2A that negatively regulates Akt [391]. Suppression of PP2A attenuated Bim expression and cell death in cerebral endothelial cells [391].

\subsection{Involvement of Bim in Regulating Immune Responses}

\subsubsection{General Aspects of Bim on Hematopoiesis}

Bim is essential for hematopoietic cell homeostasis, thymocyte negative selection and as a barrier against autoimmunity [53]. Bim is required for the deletion of autoreactive $\mathrm{T}$ and $\mathrm{B}$ cells $[36,46]$, the death of germinal center-derived memory B cells and antibody-forming cells [392] and the regulation of naïve and memory $T$ cell homeostasis [393]. While the numbes of both CD4-8pro- $\mathrm{T}$ cells and mature $\mathrm{CD} 4^{+} 8^{-}$or $\mathrm{CD} 48^{+} \mathrm{T}$ cells were $2-3$ fold higher in Bim $\mathrm{KO}$ mice than normal mice, the $\mathrm{CD}^{+} 8^{+}$ thymocytes population was only half the normal level [2]. $\mathrm{Bim}^{-/}$mice had increased number of $\mathrm{CD} 25^{\text {low }} \mathrm{Foxp} 3{ }^{+} \mathrm{CD} 4^{+}$ T-regulatory $\left(\mathrm{T}_{\text {reg }}\right)$ cells in the thymus and peripheral lymph nodes that were anergic [394]. Bim KO mice show a 2-4 fold increase in B and T cell numbers and suffer from splenomegaly and lymphadenopathy due to accumulation of excess lymphoid and myeloid cells [2]. Bim-deficient mice develop a late-onset autoimmune disease [2].

Also, apoptosis of activated $\mathrm{T}$ and $\mathrm{B}$ cells and the proper termination of immune responses are dependent on Bim [47, 395]. The Bim KO mice are unable to terminate CD8-driven immune responses after clearing a viral challenge [395] and showed impaired superantigeninduced T cell death [47]. Bcl-2 levels are downregulated in $\mathrm{T}$ cells after activation, leading to the sensitization of $\mathrm{T}$ cells to Bim following super-antigen injection [47].

Hutcheson et al. [396] studied $\mathrm{Bax}^{-/-\mathrm{Bim}^{-/}}$and $\mathrm{Bak}^{-/-\mathrm{Bim}^{-/}}$mice, and found that both have dysregulated hematopoiesis. The defects in myeloid and B-lymphoid development were more severe than those found in single $\mathrm{KO}$ mice. The thymocytes from these double $\mathrm{KO}$ mice were more resistant to apoptotic stimuli mediated by the intrinsic pathway [396]. Further studies by Hutcheson et al. [397] showed that combined deficiency of Bim and Fas resulted in early onset of systemic lupus erythematosus. Hughes et al. [398] observed that $\mathrm{Bim}^{-/} \mathrm{Fas}^{\mathrm{lpr} / \mathrm{lpr}}$ mice developed remarkably enhanced and accelerated fatal lymphadenopathy and autoimmunity compared to mice 
lacking only one of these apoptosis inducers. They further showed that shutdown of an acute $\mathrm{T}$ cell response to herpes simplex virus (HSV) involved only Bim with no contribution by Fas, whereas both pathways synergized in killing antigen-stimulated $\mathrm{T}$ cells in chronic infection with murine gamma-herpes virus [398]. Similarly, Weant et al. [399] showed that loss of both Bim and Fas function resulted in a synergistic disruption of lymphoid homeostasis, rapid-onset autoimmunity, and organ-specific blocks on contraction of antiviral immune responses. Following lymphocytic choriomeningitis virus (LCMV)-specific immune responses, double-mutant mice had 100-fold more antigen-specific memory $\mathrm{CD} 8^{+}$ $\mathrm{T}$ cells in their lymph nodes than wild-type mice [399]. $\mathrm{Bim}^{-/} \mathrm{Bmf}^{-/}$double deficiency in mice caused more B lymphadenopathy than loss of either $\mathrm{BH} 3$-only protein alone [400]. Combined loss of Bim and Bmf favored development of $\lambda$ light-chain secreting plasma cells [400]. The $\mathrm{Bim}^{-/} \mathrm{Bmf}^{-/-}$double knockout mice showed premature lethality due to vasculitits, insulitis and glomerulonephritis [400].

\subsubsection{Specific Roles of Bim in $\mathrm{T}$ cells}

Within the thymus, Bim $_{\mathrm{L} / \mathrm{EL}}$ expression was observed in the cortex where the double positive thymocytes are located, while Bim was absent from the thymic medulla [6]. Bim is required for the apoptosis of double positive thymocytes induced by high-affinity antigens [401]. In immature murine thymocytes, Bim is associated with mitochondria before stimulation and its level is not increased following CD $\varepsilon \varepsilon$ cross-linking, a treatment stimulating signals through the TCR [402]. However, $\mathrm{CD} 3 \varepsilon$ cross-linking led to rapid phosphorylation of $\mathrm{Bim}_{\mathrm{EL}}$ in $\mathrm{CD}^{+} \mathrm{CD}^{+}$thymocytes [402]. In contrast to TCR signaling, dexamethasone did not lead to Bim phosphorylation in $\mathrm{CD}^{+} \mathrm{CD}^{+}$thymocytes [402]. Bim is overexpressed in pro-apoptotic, pre-TCR-deficient thymocytes [403]. Pre-TCR signaling suppresses Bim expression through $\mathrm{PI} 3 \mathrm{~K} / \mathrm{Akt}$-mediated inhibition of FoxO3a [403]. Bim is also essential for the deletion of $\mathrm{CD} 4^{+} \mathrm{CD} 8{ }^{-} \mathrm{CD} 24^{+}$thymocytes in response to TCR ligation [404]. TCR ligation upregulates Bim expression in thymocytes and promotes the interaction of Bim with Bcl$\mathrm{xL}$, leading to thymocyte killing [46].

Bim is also required for the activation of autoreactive $\mathrm{T}$ cells [41]. Deletion of Bim in hematopoietic cells rendered mice resistant to autoimmune encephalomyelitis and diabetes, and Bim-deficient $\mathrm{T}$ cells showed diminished cytokine production. The Bim-deficient $\mathrm{T}$ cells showed defective calcium signaling upon $\mathrm{T}$ cell receptor activation, that was associated with an increase in the formation of an inhibitory complex containing Bcl-2 and the inositol triphosphate receptor (IP3R). Thus, in addition to mediating the death of auto-reactive $\mathrm{T}$ cells, Bim controls $T$ cell activation through the inositol triphosphate receptor (IP3R)/calcium/nuclear factor of activated $\mathrm{T}$ cells
(NFAT) pathway [41]. This function may explain why Bim-deficient mice do not reject their own organs despite lacking thymic negative selection [41].

Bim is required for negative selection of thymocytes recognizing tissue-restricted (TRA), but not ubiquitous (UbA), self-antigens [401, 405, 406]. In polyclonal Bim KO mice, there is an increase in anergic phenotype $\mathrm{CD}^{+} \mathrm{T}$ cells, which may indicate an increase in selfspecificities due to a block of clonal deletion [405]. Also, Bim deficiency in V $\beta 5$ transgenic mice led to impaired peripheral deletion of $\mathrm{CD}^{+} \mathrm{T}$ cells, resulting in more Recombination activating gene (RAG)-expressing, revising $\mathrm{CD}^{+} \mathrm{T}$ cells [407].

Selective clonal deletion of the $\mathrm{CD}^{+} \mathrm{T}$ cell compartment during the transition from effector to memory is accompanied by enhanced expression of Bim. Bim deficiency enables the survival of poorly functional Th1 responders that are normally eliminated during contraction [408]. $\mathrm{Bim}^{-/-} \mathrm{CD}^{+}$memory $\mathrm{T}$ cells showed deficient effector functions, poor sensitivity to antigen and an inability to respond to secondary challenge [408]. Thus, Bim plays a key role in shaping the $\mathrm{CD}^{+}$memory $\mathrm{T}$ cell repertoire, ensuring the emergence of highly functional $\mathrm{CD}^{+}$memory $\mathrm{T}$ cells and the elimination of Th1 effector cells with sub-optimal function [408].

Bim was also found to be involved in the apoptosis of effector T cells following interaction of B7-H1 (PDL1) with its receptor PD-1 [409]. B7-H1 is expressed on primary and metastatic tumor cells as well as antigenpresenting cells and exerts immunosuppressive functions on $\mathrm{CD}^{+} \mathrm{T}$ cells. Interruption of the PD-L1/PD-1 interaction using blocking antibodies to either of the two molecules has proven effective in increasing the antitumor immune responses [410]. More memory $\mathrm{CD}^{+} \mathrm{T}$ cells were generated in B7-H1-deficient mice following immunization as compared to wild-type mice [409]. At the peak of the expansion phase, $\mathrm{CD} 8^{+} \mathrm{T}$ cells expressed lower levels of Bim in the B7-H1-deficient mice than wild-type mice [409]. Bim is a key regulator of $\mathrm{T}$ cell apoptosis during the contraction phase of $\mathrm{CD}^{+} \mathrm{T}$ cell response [399]. Thus, B7-H1 negatively regulates $\mathrm{CD}^{+}$ $\mathrm{T}$ cell memory by enhancing the depletion of effector $\mathrm{CD}^{+} \mathrm{T}$ cells through upregulation of Bim [409]. Also, Kurtulus et al. [411] observed that Bim controls $\mathrm{T}$ cell memory development by limiting the survival of prememory effector cells. The absence of Bim increased the effector $\mathrm{CD}^{+} \mathrm{T}$ cell population with more memory potential, which was due to increased IL-15-dependent survival of memory precursors [411]. Although Bim was critical to limit survival of killer-cell lectin like receptor G1 (KLRG1) ${ }^{\text {hi }}$ CD127 $7^{\text {lo }}$ T effector cells, the absence of Bim enriched for KLRG $1{ }^{\text {lo }} \mathrm{CD} 127^{\text {hi }}$ pre-memory $\mathrm{T}$ cells as the response progressed [411].

Also the production of regulatory $T$ cells $\left(\mathrm{T}_{\mathrm{reg}}\right)$ was affected by Bim [37-39]. Regulatory T cells accumulate dramatically in aged mice, which is due to reduced Bim 
expression [38]. Bim expression naturally declines in peripheral wild-type $\mathrm{CD}^{+} \mathrm{T}$ cells during aging [412], which is co-incident with the increasing numbers of $\mathrm{T}_{\text {reg }}$ cells [37]. In the absence of Bim, $\mathrm{T}_{\text {reg }}$ cells accumulate rapidly, accounting for $>25 \%$ of the $\mathrm{CD}^{+} \mathrm{T}$ cell population by 6 months of age [38]. The increased $T_{\text {reg }}$ population in Bim KO mice may explain, at least in part, why these mice do not develop aggressive $\mathrm{T}$ cell-mediated autoimmune disease.

The preferential accumulation of $T_{\text {reg }}$ cells in the absence of Bim might be explained by the rapid peripheral $\mathrm{T}_{\text {reg }}$ cell turnover that depends on Bim [413] and antagonized by Mcl-1 [39]. Tischner et al. [414] observed that the longer-lived Bim-deficient $\mathrm{T}_{\text {reg }}$ cells showed reduced suppressive capacity in a model of $\mathrm{T}$ cell-driven colitis. However, Reckling et al. [415] noticed increased resistance of Bim $^{-/}$mice to persistent Leishmania major infection. The initial parasite growth and lesion development were similar in $\mathrm{Bim}^{-/}$and wildtype mice after primary Leishmania major infection [415]. At later times after infection, $\mathrm{Bim}^{-/}$mice had significantly increased Leishmania major-specific $\mathrm{CD}^{+} \mathrm{T}$ cell responses and were resistant to persistent infection [415].

Retinoic acid inhibits TCR-mediated cell death of thymocytes during negative selection by inhibiting DNA binding of Nur77 and synthesis of Bim [416]. This study showed that anti-CD3- and specific antigen-driven, but not superantigen-mediated apoptosis, involves Bim [416]. Nur77 didn't directly upregulate Bim [416], but could increase apoptosis by antagonizing Bcl-2 [417]. A recent report by Kiss et al. [418] suggests that Nur77 induces STAT-1 that, in turn, enhances Bim expression [418].

Larrubia et al. [419] observed that Bim expression in hepatitis $\mathrm{C}$ virus $(\mathrm{HCV})$-specific $\mathrm{CD}^{+}$cells was elevated in persistent $\mathrm{HCV}$-infected patients in comparison to those with resolved infection. The $\mathrm{CD} 8^{+}$cells from resolved infection showed a PD-1-CD127 (IL-7 receptor) ${ }^{+}$ phenotype and proliferated after stimulation, while the $\mathrm{CD}^{+}$cells from persistent infection patients showed a PD- $1^{+} \mathrm{CD} 127-\mathrm{Bim}^{+}$phenotype with impaired proliferation after stimulation [419]. Blocking apoptosis and the PD-1/ PD-L1 pathway enhanced their reactivity in vitro [419].

CD137L (4-1BBL), a stimulator of anti-viral CD8 $\mathrm{T}$ cell responses, led to TNFR-associated factor 1 (TRAF-1)-dependent Bim downregulation in activated virus-specific $\mathrm{CD} 8^{+} \mathrm{T}$ cells, resulting in enhanced $\mathrm{T}$ cell expansion [420]. This study confirms the studies showing that $\mathrm{CD} 137 \mathrm{~L}$ recruits TRAF1 during signaling in T cells [421] and TRAF-1 reduces Bim expression in activated $\mathrm{T}$ cells [422]. TRAF1-deficient, antigen-activated $\mathrm{T}$ cells accumulated higher levels of Bim, particularly the $\mathrm{Bim}_{\mathrm{s}}$ isoform, thereby showing reduced CD8 $\mathrm{T}$ cell response to influenza virus [422]. Bim downregulation led to increased memory T cell survival in TRAF1-deficient mice [422]. Agonistic antibodies to CD137 (e.g., Urelumab) promoted survival and activation of specific $\mathrm{T}$ cells, and increased the immune responses against melanoma [423].

\subsubsection{Specific Roles of Bim in B cells}

Bim-deficient B lymphocytes are refractory to apoptosis induced by ligation of $\mathrm{B}$ cell antigen receptor (BCR) [36]. BCR ligation promoted interaction of Bim with Bcl-2, inhibiting its survival function [36]. B cell activating factor belonging to the tumor necrosis factor family (BAFF) and A proliferation-inducing ligand (APRIL) block BCR-induced apoptosis by downregulating Bim expression [424]. BAFF induced ERK-mediated Bim phosphorylation and inhibited BCR-induced association of Bim with Bcl-2 [424]. Autoreactive B cells appear to depend on increased BAFF signaling for survival to counteract self-antigen-driven increases in Bim levels [425]. Normal B cells require BAFF both for differentiation and survival, while Bim-deficient B cells require BAFF only for differentiation [426]. IL-4 showed a unique protective effect against anti-IgM apoptotic signals on transitional B cell checkpoints by reducing Bim expression [356]. IL-4 and BAFF synergized to promote B cell maturation [356].

Although transitional immature type 1 (T1) B cell numbers are normal in Bim KO mice, T2 and follicular mature B cells are elevated and marginal zone B cells are reduced [424]. Bim loss prevented deletion of autoreactive B cells induced by soluble self-antigen and promoted accumulation of self-reactive $B$ cells developing in the presence of membrane-bound selfantigen [36]. Bim-deficient B cells were protected in vivo from superantigen-induced death and underwent persistent massive supraclonal expansion without functional impairment [427]. The microbial virulence factor protein A of Staphylococcus aureus induced specifically the $\mathrm{Bim}_{\mathrm{S}}$ isoform in B cells [427].

Bim expression is required for cross-linked anti- $\mu$ antibody-mediated apoptosis in various Burkitt's lymphoma cell lines, whereas it is not required for apoptosis triggered by soluble anti- $\mu$ antibody [83]. Following anti- $\mu$ antibody stimulation, $\mathrm{Bim}_{\mathrm{EL}}$ is phosphorylated by ERK followed by proteasomedependent degradation, a process that occurs concomitantly with de novo synthesis of $\mathrm{Bim}_{\mathrm{L}}$ and $\mathrm{Bim}_{\mathrm{S}}$ that were responsible for the pro-apoptotic effect through direct interaction with Bax [83]. This shift in Bim isoform expression was not observed when the Burkitt's lymphoma cells were exposed to TRAIL or TGF $\beta$ [83].

Craxton et al. [428] presented data suggesting a role for of Bim in regulating BCR-induced entry of B cells into the cell cycle. Bim KO B cells had reduced cell division compared to wild-type $\mathrm{B}$ cells in response to BCR, Toll-like receptor 3 (TLR3) or TLR4 signaling, whereas Bim deficiency did not affect TLR9-induced B cell division [428]. Cell cycle progression in BCR- and lipopolysaccharide (LPS)-stimulated Bim KO B cells was blocked at the $\mathrm{G}_{0} / \mathrm{G}_{1}$ stage [428]. BCR-induced 
p130 degradation and $\mathrm{pRb}$ hyperphosphorylation on Ser807/811, which are critical for $\mathrm{G}_{1}$ entry, were reduced in Bim KO compared to wild-type B cells [428].

$\mathrm{B}$ cell Sialic acid-binding immunoglobulintype lectins (Siglecs) mediate tolerance to cell surface antigens by initiating an inhibitory signal that culminates in elimination of antigen-reactive B cells. CD22 and Siglec-G are recruited to the immunological synapse by sialic acid ligands on the antigen-binding cells, producing a tolerogenic signal involving Lyn and Bim that promotes deletion of the B cells and failure of mice to develop antibodies to the antigen upon subsequent challenge [429]. This mechanism may be involved in the induction of humoral tolerance in recipients by donor-specific transfusion following organ transplantation [429].

\subsubsection{Role of Bim in Macrophages and Dendritic cells}

Bim is phosphorylated and upregulated during Tolllike receptor (TLR) stimulation of macrophages [430]. Inhibition of the MAPK p38 reduced both upregulation of Bim and apoptosis [430]. Bim upregulation was induced by LPS that stimulates TLR4, the bacterial lipopeptide $\mathrm{Pam}_{3}$ Cys that stimulates TLR2, and $\mathrm{CpG}$ oligodeoxynucleotide that stimulates TLR9 [430]. It should be noted that upregulation of Bim per se was insufficient in inducing apoptosis, that required an additional signal [430]. The adaptor protein MyD88 was both necessary and sufficient for the induction of Bim [430]. FoxO3a seems to be involved in Bim induction, as it translocates into the nucleus upon TLR stimulation [430]. Of note, $\mathrm{Bim}^{-/}$macrophages were resistant to E. coli phagocytosis-induced apoptosis [430]. TLR stimulation might also promote Bim degradation through ERKmediated phosphorylation of Bim at Ser55, Ser65 and Ser100 [431].

Bim KO mice had an increased number of dendritic cells [432]. Bim is expressed at low levels in dendritic cells, but is significantly upregulated by signaling from CD40 or TLRs [432]. Simultaneously with the upregulation of Bim, the anti-apoptotic Mcl-1 is also upregulated by TLR signaling, that antagonizes the pro-apoptotic function of Bim during the early stages of dendritic cell activation [432]. GM-CSF treatment of dendritic cells led to reduced Bim expression and increased dendritic cell survival [432]. Bim-deficient dendritic cells showed decreased spontaneous cell death and induced more robust $\mathrm{T}$ cell activation, thus being more immunogenic [432]. Adoptive transfer of Bim-deficient dendritic cells led to the induction of autoantibody production that may contribute to the spontaneous systemic autoimmunity observed in Bim KO mice [432].

\subsubsection{Role of Bim in Granulocytes}

Myeloid cell life span is substantially increased in Bim KO mice, and blood neutrophil counts in these animals are increased by a factor of 2.5 [2]. Both immature and mature neutrophils express the three major isoforms of Bim: $\operatorname{Bim}_{\mathrm{EL}}, \mathrm{Bim}_{\mathrm{L}}$ and $\operatorname{Bim}_{\mathrm{S}}$ [433]. Bim deficiency, like Bcl-2 overexpression, renders granulocytes resistant to cytokine withdrawal and cytotoxic drugs such as etoposide and paclitaxel, but does not have any impact on FasLinduced apoptosis [357].

The neutrophil survival factor GM-CSF does not only prevent the normal time-dependent loss of the anti-apoptotic proteins Mcl-1 and Bcl2A1 in neutrophils important for their survival, but also increases Bim transcription and $\mathrm{Bim}_{\mathrm{EL}}$ expression by a mechanism dependent on NFאB [434]. It is possible that neutrophils can tolerate the increase in Bim expression because of the corresponding increase in anti-apoptotic Bcl-2 members [434]. Increased Bim mRNA expression was observed in neutrophils in lung lavage from patients with ventilatorassociated pneumonia [434] and in neutrophils from blood of septic patients [433]. The paradoxical increase in Bim expression by the neutrophil survival factor may function to facilitate rapid apoptosis at the termination of inflammation [434]. In vitro, GM-CSF primes neutrophils for TNF $\alpha$-mediated killing [434]. A similar pro-apoptotic counter-regulation of GM-CSF on neutrophils was observed by Andina et al. [433]. A lack of Bim resulted in a much higher efficacy of the survival cytokines IL-3 and GM-CSF to block neutrophil apoptosis [433]. Bauer et al. [435] showed a similar paradox that LPS stimulation of mouse bone marrow neutrophils reduced spontaneous apoptosis, but at the same time increased Bim expression.

\subsubsection{Role of Bim in NK cells}

Natural killer (NK) cells undergo antigendriven expansion to become memory cells after mouse cytomegalovirus (MCMV) infection. $\mathrm{Bim}^{-/} \mathrm{Ly}_{4} 9 \mathrm{H}^{+} \mathrm{NK}$ cells expanded normally during the early infection phase, but showed reduced contraction after the expansion phase leading to higher NK cell numbers than wild-type cells [436]. The inability to reduce the effector pool leads to larger $\mathrm{Bim}^{-/} \mathrm{NK}$ memory subsets, which displays a less mature phenotype $\left(\mathrm{CD} 11 \mathrm{~b}^{\text {lo }}, \mathrm{CD} 27^{+}\right)$and lower levels of NK cell memory-associated markers KLGR1 and Ly6C [436]. Bim ${ }^{--}$memory NK cells showed a reduced response to the MCMV-encoded ligand m157-mediated stimulation and were less effective than wild-type NK cells in protecting against MCMV infection [436].

\subsubsection{Role of Bim in Mast Cells}

Bcl-xL and Bim were both induced upon FceRI activation of mast cells [358]. Bim deficiency prevented cytokine withdrawal-induced mast cell apoptosis, suggesting for an essential role of Bim in this cell death process [358]. The Kit receptor tyrosine kinase signaling via SHP2 and ERK was found to be important for the downregulation of Bim required for mast cell survival [437]. SHP2 ${ }^{--}$mast cells failed to mount an IgE-mediated late phase cutaneous response [437]. $\mathrm{PGE}_{2}$ increases mast cell death during cytokine deprivation by augmenting Bim expression [438]. Influenza A virus induces apoptosis of 
mast cells that concurs with upregulation of Bim [439].

\subsubsection{Role of Bim in Megakaryopoiesis}

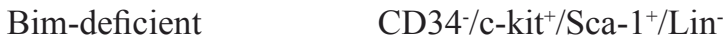
hematopoietic stem cells and megakaryocytes are resistant to apoptosis induced by cytokine depletion [440]. Platelet recovery after 5-fluorouracil-induced thrombocytopenia is delayed in Bim KO mice, which is reflected in reduced number of megakaryocytes [440]. Bim-deficient c-Kit ${ }^{+}$ Lin ${ }^{-}$progenitor cells poorly proliferate and differentiate into $\mathrm{CD} 41^{+}$cells in response to thrombopoietin, but once differentiated into megakaryocytes, these cells mature normally. The transition from $\mathrm{G}_{1}$ to $\mathrm{S}$ phase is delayed in Bim-deficient hematopoietic stem cells, suggesting a role for Bim in regulating cell cycle progression in hematopoietic progenitors during megakaryopoiesis [440].

\subsection{Involvement of Bim in Neuronal Differentiation and Apoptosis}

Bim and Puma have been shown to be involved in the death of cells that have been newly generated during neurogenesis in the adult hippocampus [441]. Bimdeficient mice showed similar rates of precursor cell proliferation in the mouse hippocampus as wild-type mice, but the survival of adult-born cells in the dentate gyrus was increased [441]. There was no change in the early markers of neuronal differentiation, while the differentiation of newly generated cells into a neuronal phenotype was accelerated in the Bim-deficient mice [441]. These findings suggest that besides a role in regulating neuronal progenitor cell survival, Bim may inhibit neuronal differentiation [441]. Also the anti-apoptotic Bcl-2 has been implicated in neuronal differentiation [442] and promotes axonal outgrowth of retinal ganglion cells [443]. As Bim binds Bcl-2, it could be that Bim determines the available Bcl-2.

Cellular stresses resulting from extracellular stimulation by $\mathrm{H}_{2} \mathrm{O}_{2}$ or $\beta$-amyloid promote hyperactivation of cyclin-dependent kinase 5 (Cdk5) [444]. Cdk5 prevents cell death of postmitotic cortical neurons through phosphorylation of FoxO1 at Ser249, thus preventing FoxO1-mediated upregulation of Bim [444].

Dysfunction of mitochondrial complex I associated with Parkinson's disease leads to degeneration of dopaminergic neurons of the substantia nigra pars compacta (SNpc) through a mechanism involving JNK-dependent activation of Bim with consequent Bax translocation to the mitochondria and apoptosis [445]. Analysis of post-mortem human brain samples from Parkinson's disease patients has shown elevated JNK activation [446].

Activation of Notch contributes to ischemic neuronal cell death [447]. Inhibition of the Notchactivating enzyme $\gamma$-secretase protected against ischemic neuronal cell death by reducing Bim expression [447]. Treatment of mice with the $\gamma$-secretase inhibitor compound $\mathrm{E}$ reduced infarct size and improved functional outcome in a model of focal ischemic stroke [447]. The upregulation of Bim by active Notch in neuronal cells was mediated by NFאB [447]. Of note, constitutively active Notch1 downregulates Bim expression in other cell types (e.g., T cell acute lymphoblastic leukemia) through induction of c-Myc and repression of PTEN that leads to the activation of the PI3K/Akt/mTOR pathway [294]. The Notch1 transcriptional function in $\mathrm{T}$ malignancies and pancreatic cancer was supported by Bcl-2 that fortified drug resistance [199, 448].

\subsection{Involvement of Bim in Diabetes}

Bim contributes to cytokine- [183, 188], virus- [449] and high glucose-induced $[450,451]$ pancreatic $\beta$-cell apoptosis. Bim can be regulated by pro-inflammatory cytokines at the transcriptional level through STAT1 [183] and by phosphorylation [188]. Puma and Bax are also involved in high glucose-induced apoptosis of islet cells $[450,451]$. Loss of Bid, Noxa or Bak had no impact on glucose-induced apoptosis [451]. Islets deficient in both Bim and Puma, but not Bim or Puma alone, were protected from killing induced by the mitochondrial reactive oxygen species donor rotenone [450].

Non-obese diabetic (NOD) mice that develop spontaneous autoimmune diabetes, show decreased thymic clonal deletion associated with diminished induction of Bim and Nur77 [452]. These mice show defective thymic enrichment of $\mathrm{CD} 4{ }^{+} \mathrm{CD} 25^{+} \mathrm{T}_{\text {reg }}$ cells [452]. Bim deficient NOD mice developed less insulitis and were protected from diabetes despite substantial defects in the deletion of autoreactive thymocytes [40]. Bim deficiency didn't impair effector $\mathrm{T}$ cell function, but NOD Bim${ }^{-/}$mice had increased numbers of antigen-specific $\mathrm{T}_{\text {reg }}$ cells both in the thymus and peripheral lymphoid tissues [40]. It is likely that the absence of Bim in the $\beta$-cells prevents their cell death, thereby avoiding the trigger of the immune system, which often happens due to cell death-released factors such as High-mobility group box 1 (HMGB1) that activates the receptor for advanced glycation end products (RAGE) and spliceosome-associated protein 130 (SAP130) activating C-type lectin 4e (Clec4e) [453].

The Bim gene Bcl2111 lies within the Idd13 diabetes susceptibility locus [40, 452]. As described in Sections 3.1.3 and 3.1.1.7, diabetes-related GLIS deficiency leads to preferential upregulation of the $\mathrm{Bim}_{\mathrm{S}}$ isoform [233], and diabetes-related PTPN2 deficiency leads to increased activation of STAT-1 resulting in increased Bim transcription [183]. Also, other candidate risk genes for type 1 diabetes have been described that protects $\beta$-cells from apoptosis through prevention of Bim upregulation. For instance, deficiency in the basic leucine zipper transcription factor 2 (BACH2) leads to increased phosporylation of JNK1 by mitogen-activated protein kinase kinase 7 (MKK 7 ) and downregulation of PTPN2. These changes, in turn, led to increased Bim expression [454]. The candidate gene cathepsin H prevents 
$\beta$-cell apoptosis by suppressing JNK and p38 signaling and reducing Bim expression [455]. Pro-inflammatory cytokines decreased the expression of cathepsin $\mathrm{H}$ in human islets, and overexpression of cathepsin $\mathrm{H}$ protected $\beta$-cells against cytokine-induced apoptosis [455].

Islets of human donors with type 2 diabetes had higher mRNA levels of Bim and Puma, suggesting that these pro-apoptotic proteins contribute to $\beta$-cell death [450]. High concentrations of glucose led to increased expression of the transcription factor CHOP (C/ERB homologous protein) [450], that co-operates with $\mathrm{FoxO} 3 \mathrm{a}$ to regulate Bim and Puma expression [181]. The increased apoptosis of $\beta$-cells in pancreatic duodenal homeobox-1 (Pdx1)-haploinsufficient mice could be prevented by simultaneous knockout of either Bim or Puma [456]. Both Bim and Puma expression is upregulated in $\beta$-cells following Pdx1 suppression [456]. Similarly, Bim mediates $\beta$-cell death upon Insulin receptor substrate 2 (IRS2) deficiency [457]. Dexamethasone induced Pdx1 downregulation and Bim activation in $\beta$-cells by a mechanism dependent on glucocorticoid receptor activation, but independent of FoxO1 [458]. Diabetogenic conditions lead to the upregulation of Mst1 in $\beta$-cells of both human and mouse islets, resulting in the induction of Bim expression and apoptosis [459]. Mst1 phosphorylates Pdx1 at Thr11, resulting in ubiquitination and degradation of Pdx-1 [459].

\subsection{Involvement of Bim in Osteoarthritis}

Osteoarthritis is a chronic degenerative joint disorder characterized by increased apoptosis of chondrocytes. Bim is upregulated in chondrocytes obtained from the articular cartilage of osteoarthritis patients and in cultured mouse chondrocytes treated with IL-1 $\beta$ [460]. The cytokine IL$1 \beta$ induces chondrocyte apoptosis through activation of the JNK-c-Jun pathway that leads to induction of Bim expression [460]. Genetic knockdown of Bim reduced chondrocyte apoptosis, suggesting a role for Bim [460].

\subsection{Involvement of Bim in Rheumatoid Arthritis}

The expression of Bim was reduced in macrophages from synovial tissue of patients suffering from rheumatoid arthritis [461]. Macrophages from Bim KO mice displayed elevated expression of inflammation markers and secreted more IL-1 $\beta$ in response to LPS or thioglycollate, suggesting for a role of Bim in limiting the activation of macrophages [461]. A Bim-BH3 mimetic (TAT-BH3) peptide induced apoptosis of myeloid cells and reduced the symptoms of arthritis in mice treated with $\mathrm{K} / \mathrm{BxN}$ serum [461].

\subsection{Involvement of Bim in Liver Damage}

A fine rheostatic balance between the anti- and proapoptotic multidomain Bcl-2 family proteins controls hepatocyte apoptosis in the healthy liver. Bim and Bid are functionally active, but are restrained by the anti-apoptotic Bcl-2 family proteins under physiological conditions
[462]. Spontaneous hepatocyte apoptosis in Bcl-xL- or Mcl-1-knockout mice was ameliorated by simultaneous Bim deletion [462]. Hepatocyte apoptosis caused by the BH3 mimetic ABT-737 was completely prevented in Bim/Bid double knockout mice [462]. The Wnt/ $\beta$-catenin signaling pathway protects mouse liver against oxidative stress-induced apoptosis through SGK1-mediated inhibition of FoxO3, resulting in reduced Bim expression [463]. Acetaminophen (Paracetamol) induced apoptosis of hepatocytes through JNK-mediated upregulation of Bim that was aggravated by simultaneous stimulation by TRAIL [464]. TRAIL-deficient or Bim-deficient mice were protected from acetaminophen-induced liver damage [464]. Similarily, TRAIL enhanced Fas-induced hepatocyte apoptosis through activation of JNK and Bim [465].

Hepatocyte lipoapoptosis caused by exposure of hepatocytes to saturated free fatty acids (e.g., palmitic and stearic acids) is mediated by FoxO3a-dependent Bim induction [466]. Free fatty acids induce the expression of PP2A that is responsible for FoxO3a dephosphorylation and activation [466]. Free fatty acids also activate JNK that increases Bim activity [467]. Knockdown of Bim prevented hepatocyte apoptosis induced by free fatty acids [467]. Fatal hepatitis mediated by TNF $\alpha$, produced in response to LPS and the transcriptional inhibitor $\mathrm{D}(+)$ galactosamine, could be prevented by the simultaneous deletion of Bid and Bim [468]. Palmitic acid, but not oleic acid, induced the degradation of the Kelch-like ECHassociated protein-1 (Keap-1) in hepatocytes through autophagy in a p62-dependent mechanism [469]. The reduced expression of Keap-1 led to JNK1-dependent upregulation of Bim and Puma and induction of hepatocyte apoptosis [469].

Bim is involved in the elimination of virusspecific $\mathrm{T}$ cells that have been activated by hepatocytes following hepatitis $\mathrm{B}$ and $\mathrm{C}$ virus (HBV/HCV) infections [470]. This leads to peripheral immunological tolerance towards the virus, contraction of the immune response and prevention of immune cell-mediated liver damage [470]. Bim especially promotes the elimination of $\mathrm{CD} 127^{\text {low }}$ expressing virus-specific $\mathrm{T}$ cells [471]. CD127 $7^{\text {high }} \mathrm{T}$ cells receive survival signals delivered by IL-7, leading to upregulation of Mcl-1 that captures Bim, thereby preventing apoptosis while promoting resolution of the viral infection [472]. Thus, the CD127 phenotype of the virus-specific $T$ cells affects $T$ cell reactivity by regulating apoptosis determined by the Bim/Mcl-1 balance.

Liver-activated $\mathrm{CD}^{+} \mathrm{T}$ cells showed increased expression of Bim and caspase 3, making the $\mathrm{T}$ cells prone to apoptosis following intrahepatic activation [473]. $\mathrm{T}$ cells deficient for Bim survived following intrahepatic activation [473]. $\mathrm{HBV}$-specific $\mathrm{CD} 8^{+} \mathrm{T}$ cells from patients with chronic infection showed higher Bim expression than those with resolved infection [474]. Blocking Bimmediated apoptosis enhanced the recovery of HBV-specific 
$\mathrm{CD}^{+} \mathrm{T}$ cells [474]. The few surviving HBV-specific $\mathrm{CD} 8^{+} \mathrm{T}$ cells were $\mathrm{CD} 127^{\mathrm{hi}}$ and expressed elevated levels of Mcl-1, suggesting that they were amenable to IL-7mediated rescue from apoptosis [474].

During persistent lymphocytic choriomeningitis virus (LCMV) infection, Bim was important for the specific elimination of $\mathrm{CD} 8^{+} \mathrm{D}^{\mathrm{b}} \mathrm{NP} 396-404^{+} \mathrm{T}$ cells [475]. Bim played a dual role in the development of LCMVinduced, T cell-mediated hepatitis [353]. Absence of Bim in parenchymal cells attenuated liver damage, while loss of Bim in the lymphoid compartment enhanced hepatitis [353]. In Bim KO mice, the effect of Bim deficiency in the lymphoid compartment was counterbalanced by the reduced sensitivity of Bim KO hepatocytes to T cellinduced apoptosis, resulting in the protection of the mice from hepatitis [353]. The signal adaptor TNFR-associated factor 1 (TRAF1) negatively correlates with Bim and it contributes to $\mathrm{CD} 8 \mathrm{~T}$ cell-mediated control of chronic viral infections. 4-1BB (CD137), a TNFR family member implicated in prolonging the survival of activated and memory CD8 T cells, activates ERK through association with TRAF1 and TRAF2, resulting in accelerated degradation of Bim [476]. TRAF1 is specifically lost from virus-specific CD8 T cells during the chronic phase of infection with human immunodeficiency virus (HIV) or LCMV [477]. TGF $\beta$ induces the posttranslational loss of TRAF1, whereas IL-7 restores TRAF1 expression [477]. Thus, IL-7 may have therapeutic applications in the treatment of chronic viral illnesses.

\subsection{Role of Bim in Mammary Lumen Formation}

Epithelial cells organize into cyst-like structures that contain a spherical monolayer of cells that enclose a central lumen. Reginato et al. [325] showed a requirement for Bim in selectively triggering apoptosis of the centrally localized acinar cells, leading to controlled lumen formation. In a three-dimensional basement membrane culture model in which mammary epithelial cells form hollow, acinus-like structures, Bim was not detectable during early stages of mammary acinar morphogenesis, but was later highly upregulated in acinar cells [325]. Inhibition of Bim expression by RNA interference transiently blocked luminal apoptosis and delayed lumen formation [325]. Oncogenes that induce acinar luminal filling, such as ErbB2 and v-Src, suppressed Bim expression through ERK-dependent pathway [325]. Bim is also involved in the death of alveolar cells during involution of the mammary gland after cessation of lactation [478].

\subsection{Radioresistance of Mesenchymal Stromal Cells (MSCs)}

Mesenchymal stromal cells (MSCs) are intrinsically resistant to $\gamma$-irradiation-induced cell death by expressing high levels of DNA damage response proteins and high levels of Bcl-2 and Bcl-xL together with low Bim expression [479]. This property of MSCs has implications for allogeneic bone-marrow transplantation, graft-versushost disease and cancer treatment.

\subsection{Involvement of Bim in Cancer}

Reduced Bim expression is a hallmark for carcinogenesis. Tumor cells have evolved different mechanisms to suppress Bim expression and/or activity thereby overcoming the apoptotic barrier that else would have led to their eradication. The efficacy of many anticancer drugs depend on Bim, and insufficient Bim induction or Bim function is often an underlying cause of therapy failure. Many cancer cells have developed one or more mechanisms for preventing Bim from acting, intervention of which may result in the reactivation of the apoptotic process. Determination of the specific survival dependency pathway in each cancer case is important for choosing the right targeting drug therapy.

\subsubsection{A Tumor Suppressive Function of Bim}

Several studies suggest that Bim functions as a tumor suppressor. In mice, inactivation of one allele of Bim accelerates Myc-induced B cell leukemia [480]. In this experimental system, Bim is induced by Myc, and inactivation of one Bim allele was sufficient for Mycinduced tumor development. Whereas the $\mathrm{p} 19^{\text {Arf }} / \mathrm{p} 53$ pathway is frequently mutated in tumors arising in $\mathrm{Bim}^{+/+}$ E $\mu$-Myc mice, it was unaffected in most Bim-deficient tumors, indicating that Bim reduction is an effective alternative to loss of p53 function [480]. Similarly, Bim deficiency in mice overexpressing the $\mathrm{E} \mu$-vAbl oncogene accelerated the development of plasmacytomas [481]. The v-Abl-expressing plasmacytomas frequently harbor a rearranged c-Myc gene [481]. Another example is the formation and growth of tumors derived from baby mouse kidney epithelial (BMK) cells transformed by E1A and dominant negative $\mathrm{p} 53$, that is facilitated by simultaneous Bim deficiency [482], suggesting a role for Bim in preventing epithelial cancer cell formation. These authors [482] also showed that Bim-deficiency led to paclitaxelresistant tumor cells.

An interesting study by Merino et al. [163] showed that Bim deficiency in PyMT (MMTV-Polyoma middle-T) female mice didn't affect primary breast tumor growth, but rather increased the survival of metastatic cells within the lung. They further showed a correlation between Bim expression and the EMT transcription factor SNAI2 at the proliferative edge of the tumor. Chromatin immunoprecipitation analysis suggests that Bim is a target of SNAI2 [163]. These data suggest a role for Bim in the suppression of breast cancer metastasis.

Bim is frequently eliminated in human cancer, providing a growth advantage to the tumor cells. For instance, homozygous deletions of the Bim locus have been observed in mantel cell lymphomas and methylation of the bim promoter has been found in certain Burkitt's lymphoma and diffuse large B cell lymphoma [246, 483]. Bim is downregulated in a subset of colorectal 
cancers through Cox $2 / \mathrm{PGE}_{2}$ signaling that activates the c-Raf/MEK/ERK1/2 pathway [484]. Treatment of Cox2expressing colorectal carcinoma with selective Cox2 inhibitors induced Bim expression [484]. Downregulation of Bim expression was associated with tumor progression towards an anchorage-independent phenotype [484].

When the ERK pathway is constitutively activated in tumor cells, Bim is degraded, and confers chemoresistance. This was demonstrated by Tan et al. [482] where apoptotic sensitivity to paclitaxel could be restored in H-Ras/ERKdependent tumor cells, by treatment with the proteasome inhibitor bortezomib that promoted Bim-dependent tumor regression [482]. Bortezomib also sensitized prostate and colon cancer cells to TRAIL-mediated cell death through a mechanism dependent on Bik and Bim [485]. Similarly, Bim levels are low in NSCLC cells harboring activating EGFR mutations [306-309]. Inhibition of EGFR tyrosine kinase activity by drugs such as gefitinib, results in Bim ${ }_{\mathrm{EL}}$ accumulation and induction of apoptosis.

Metastatic melanomas had lower levels of Bim than dysplastic nevi [486]. Reduced Bim expression was significantly correlated with poor 5-year survival of melanoma patients [486]. The single point mutation $\mathrm{V} 600 \mathrm{E}$ in B-Raf, frequently observed in melanoma, inhibited Bim expression through ERK-dependent phosphorylation and degradation $[314,487]$. This might be one mechanism for the anti-apoptotic actions of B-Raf ${ }^{\mathrm{V} 600 \mathrm{E}}$ [487].

Petrocca et al. [175] observed that Bim expression is higher in gastric primary tumors than normal tissues. This might be due to upregulation of Bim by oncogenic stress stimuli [480]. Bim ${ }_{\mathrm{EL}}$ and $\mathrm{Bim}_{\mathrm{L}}$ were also found to be expressed at higher levels in prostate cancer cells than normal prostate tissue [30].

\subsubsection{Role of Bim in Anoikis}

Epithelial cells usually need to attach to extracellular matrix (ECM) or interact with neighboring cells in order to receive integrin signals required for survival. When detached from substratum, the epithelial cells undergo apoptosis through a process termed anoikis. In order to survive, a metastatic cell needs to acquire anchorage independence to overcome anoikis. Bim plays a key role in the anoikis of a variety of tumor cells, such as breast cancer, lung cancer, osteosarcoma, fibrosarcoma, and melanoma [488-490]. Bim is strongly induced after cell detachment as a result of attenuated integrin signal with consequent reduced ERK activity [489]. Overexpression of EGFR results in the maintenance of ERK activity following detachment, thus preventing Bim induction and anoikis [489]. The cell surface glycoprotein mesothelin, which is overexpressed in various tumors, promotes anchorage-independent growth and suppresses Bim expression via ERK activation in human breast cancer cells [490]. Also, the focal adhesion protein PINCH-1, which is a cytoplasmic component of cell-extracellular matrix adhesions, can activate ERK-mediated Bim degradation, besides suppressing Bim transcription [210]. Giannoni et al. [491] observed that integrin engagement leads to the production of reactive oxygen species (ROS) that are responsible for the redox-mediated activation of Src that transphosphorylates EGFR in a ligand-independent manner. EGFR, in turn, activates ERK and Akt signaling pathways that culminate in the degradation of Bim [491]. Overexpression of the receptor tyrosine kinase HER2/ neu (ErbB2) in breast cancer, led to increased HIF-1 $\alpha$ expression, increased ERK and Akt activities, reduced Bim expression and resistance to anoikis [224]. The transcription factor Pokemon renders liver cells resistant to anoikis through suppression of Bim transcription [492]. G1/S cell cycle arrest induced by overexpression of $\mathrm{p} 16^{\mathrm{INK} 4 \mathrm{a}}, \mathrm{p} 21^{\text {Cip }}$ or $\mathrm{p} 27^{\mathrm{Kip}}$ leads to sustained ERK activation, Bim suppression and anoikis resistance [493]. However, overexpression of CXCL12 in colorectal carcinoma led to increased sensitivity to anoikis, enhanced expression of Bim and reduced metastatic potential [494].

During mammary morphogenesis, cells that become detached from the extracellular matrix undergo apoptosis, leading to the formation of the mammary lumen [495]. In contrast to normal mammary epithelial cells that require attachment to ECM for survival, inflammatory breast cancer (IBC) cells evade anoikis. ErbB2/EGFR signaling through the ERK/MAPK pathway protects the cells from anoikis by facilitating the formation of a protein complex containing Bim ${ }_{\mathrm{EL}}$, LC8 and Beclin-1 [496]. This complex forms as a result of $\mathrm{Bim}_{\mathrm{EL}}$ phosphorylation on Ser59,

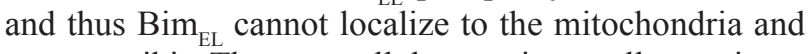
cause anoikis. The extracellular matrix metalloproteinase inducer CD147 (EMMPRIN) confers resistance to anoikis through inhibition of Bim, supposedly through the ERK pathway [497]. Hypoxic conditions, that prevail when the lumen of ducts is filled with breast carcinoma cells, inhibit anoikis through suppressing Bim and Bmf expression in the carcinoma cells [495]. The hypoxia-mediated anoikis protection is associated with increased activation of the EGFR/ERK signaling pathway and requires the hypoxiaactivated transcription factor HIF-1 $\alpha$ [495]. HIF-1 $\alpha$ expression predicts poor therapeutic response and clinical outcome in human breast cancers [498] and contributes to resistance to the tyrosine kinase inhibitor Lapatinib in ErbB2-positive breast cancer cells [499]. Downregulation of Protein tyrosine kinase 6 (PTK6), a non-receptor tyrosine kinase highly expressed in $\mathrm{HER} 2^{+}$breast cancer, led to p38 activation, Bim induction and apoptosis of Lapatinib-resistant HER2 ${ }^{+}$breast cancer cells [500].

\subsubsection{Bim Expression in Leukemia and Implications for Chemotherapy}

The Bcr-Abl fusion protein overexpressed in $\mathrm{CML}$ suppresses Bim expression [305]. The Bcl-Abl kinase inhibitor imatinib (Gleevec/STI-571) induces killing of $\mathrm{Bcr}_{-} \mathrm{Abl}^{+} \mathrm{CML}$ through a mechanism that depends on Bim 
and Bad, but not Bmf or Puma [304, 305, 501]. Cytokines antagonized imatinib- and nilotinib-induced apoptosis that was related to reduced Bim accumulation [501]. Drug resistance due to loss of Bim could be overcome by the BH3 mimetic ABT-737 [304].

Similarly, insulin-like growth factor 1 (IGF-1), which acts as a growth and survival factor in multiple myeloma (MM), downregulates Bim expression in these malignant cells [247]. IGF-1 prevented Bim transcription through Akt-mediated inactivation of FoxO3a, and increased proteasomal degradation of $\mathrm{Bim}_{\mathrm{EL}}$ by activating MAPK [247]. In addition, IGF-1 reduced histone $\mathrm{H} 3$ tail Lys9 (H3K9) acetylation and increased H3K9 dimethylation, both contributing to Bim silencing [247]. The deacetylase inhibitor LBH589 induced Bim expression [247]. High levels of Baculoviral inhibitor of apoptosis repeat-containing 5 (BIRC5/Survivin) expression in multiple myeloma cells correlates with reduced Bim transcription in response to IL-6 deprivation and shorter overall survival of patients [502].

Bim was shown to be epigenetically silenced in $\mathrm{NPM} / \mathrm{ALK}^{+}$anaplastic large cell lymphoma (ALCL) [503]. The Bim silencing involved the recruitment of the methyl-CpG-binding protein MeCP2 and the SIN3a/ histone deacetylase 1/2 (HDAC1/2) co-repressor complex [503]. The deacetylase inhibitor (HDACi) Trichostatin A restored histone acetylation, with concomitant upregulation of Bim [503]. While NPM/ALK induces de novo Bim 5'UTR methylation, its later silencing or inhibition by using crizotinib (PF02341066) did not lead to reacetylation of the Bim locus [503].

Treatment resistance in $\mathrm{T}$ cell acute lymphoblastic leukemia (T-ALL) is associated with PTEN deletions which result in the activation of the PI3K-Akt pathway, and Notch1-mediated c-Myc overexpression [504, 505]. Treatment with c-Myc or PI3K/Akt pathway inhibitors induced Bim upregulation and apoptosis, indicating that Bim is repressed downstream of c-Myc and PI3K/Akt in high-risk T-ALL [506]. Restoring Bim function in human T-ALL cells using a stapled peptide mimetic of the Bim $\mathrm{BH} 3$ domain had beneficial therapeutic effect [506].

Multiple myeloma cells often express high basal Bim levels that are important for bortezomib-induced apoptosis [507]. Repeated exposure to bortezomib led to marked Bim downregulation, Mcl-1 upregulation and drug resistance $[507,508]$. The drug resistance could be overcome by combining HDAC inhibitors that upregulate Bim, with ABT-737 that releases Bim from the antiapoptotic proteins [507]. Chloroquine, that interrupts the autophagic process, could further enhance the cytotoxic effect [507]. 3-Phosphoinositide protein kinase 1 (PDPK1) that is frequently activated in multiple myeloma cells, suppresses Bim expression and is associated with reduced overall survival [509]. IL-6 and IGF-1 in the microenvironment further promote drug resistance through activation of the PI3K/Akt pathway that leads to Bim downregulation and Mcl-1 upregulation [86]. Similarily, the CD28 receptor on multiple myeloma cells promote survival following interaction with its ligand CD80/CD86 on dendritic cells that is related to PI3K activation and Bim downregulation [510]. Interruption of the CD28CD80/CD86 interaction sensitized multiple myeloma cells to chemotherapy [510]. Multiple myeloma cells also

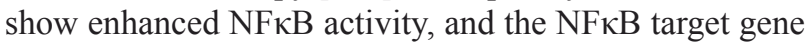
YY-1 is often hyperexpressed resulting in transcriptional repression of Bim transcription [205].

B cell receptor (BCR)- and microenvironmentalderived signals promote survival of chronic lymphoblastic leukemia (CLL) cells through increased proliferation and decreased apoptosis. Surface IgM stimulation increased phosphorylation of $\mathrm{Bim}_{\mathrm{EL}}$ and/or $\mathrm{Bim}_{\mathrm{L}}$ by an MEK1/2dependent mechanism [511]. Other studies have shown that BCR engagement in CLL leads to Akt activation in addition to ERK1/2 activation, both pathways being prosurvival [512]. CLL cells are also characterized by high expression of Mcl-1 that antagonizes the pro-apoptotic effects of Bim [511, 512], and high Mcl-1 expression is associated with poor clinical outcome [513]. This is in contrast to normal B cells where Bim mediates BCRinduced apoptosis [36], unless the MEK/ERK signaling pathway is activated by mitogenic stimuli [514]. Bim is also highly sequestered to Bcl-2 in CLL cells that make Bim rapidly available upon treatment with the $\mathrm{BH} 3$ mimetics ABT-737 [515]. Also, ALL cells with Bcl-2 dependency respond well to ABT-737 [516].

\subsubsection{Role of Bim in Glucocorticoid-Induced Apoptosis}

Glucocorticoids are critical components of combination chemotherapy regimens in pediatric acute lymphoblastic leukemia (ALL). Bim is crucial for mediating cell death in thymocytes, chronic lymphocytic leukemia, T acute lymphoblastic leukemia and multiple myeloma cells induced by glucocorticoids [14, 21, 49, $63,158,517-520]$, and its expression correlates with therapy response in lymphoid malignancies [518, 521, 522]. Glucocorticoid resistance in ALL xenografts was associated with failure to upregulate Bim expression after dexamethasone [522]. Overexpression of Bcl-2 and other anti-apoptotic proteins antagonizes glucocorticoid-induced apoptosis [14, 64]. Bcl-2-dependent resistance could be overcome by staurosporine through a Nur77-dependent mechanism [64]. Glucocorticoid resistance in xenografts and patient biopsies correlated with decreased histone $\mathrm{H} 3$ acetylation [522]. Glucocorticoids also lead to apoptosis of other cell types such as osteoblasts and insulin-producing $\beta$-cells through upregulation of Bim [458, 523].

The glucocorticoid-induced Bim expression is indirectly mediated through activation of transcription factors such as FoxO3, c-Jun and Runx1 [159, 524, 525]. Another transcription factor upregulated by glucocorticoids that may promote Bim expression is the bZIP transcriptional repressor gene E4BP4 [526- 
528]. While FoxO3 is uttermost important for the glucocorticoid-induced upregulation of $\mathrm{Bim}, \mathrm{FoxO} 3$ may also transactivate glucocorticoid-induced leucine zipper (GILZ) [529], which antagonizes FoxO transcription factors through Chromosome region maintenance 1/ Exportin 1 (CRM1)-dependent nuclear exclusion of these proteins [530]. GILZ induction may therefore serve as a negative feedback mechanism. Similarly, the glucocorticoid-response gene PLZF/ZBTB16, act as a repressor on Bim-transcription [531]. Interestingly, Jing et al. [518] observed a differential glucocorticoid response in sensitive and resistant pediatric ALL patient-derived xenografts. They demonstrated a novel glucocorticoid receptor binding site in a Bim intronic region (IGR) that was engaged only in the dexamethasonesensitive xenografts [518]. The absence of GR binding at the Bim IGR was associated with Bim silencing and dexamethasone resistance [518]. Moreover, they show that the glucocorticoid receptor transactivates KLF13, which repressed Myb expression only in the sensitive cells [518]. Sustained Myb expression in resistant xenografts resulted in maintenance of Bcl-2 expression and inhibition of apoptosis [518]. The presence of the detoxification enzyme Glutathione S-Transferase mu 1 (GSTM1) inhibits dexamethasone-induced apoptosis in lymphoblastic leukemia through suppression of Bim [532].

The upregulation of Bim per se is insufficient for initiating apoptosis. Bim needs to be activated. One activation mechanism is through interaction with GSK3, which is essential for glucocorticoid-induced apoptosis [63]. Protein kinases that inactivate GSK3 confer resistance to glucocorticoid-induced apoptosis [63]. Sensitization can be achieved by inhibiting Src, PI3K or Akt [63]. Similarly, the mTOR inhibitor rapamycin may sensitize T-ALL and MM cells to glucocorticoidinduced apoptosis through upregulation of Bim and downregulation of Mcl-1 [519, 533, 534]. Proteasomal inhibition may increase Bim expression [535], and therefore forms the rationale for combining bortezomib with glucocorticoids in the treatment of hematopoietic malignancies [517, 536]. However, Bortezomib does not only stabilize Bim, but also Mcl-1, that outranges the proapoptotic effect of Bim, thus leading to therapy resistance $[537,538]$.

\subsubsection{Bim Expression in Carcinoma and Implication for Chemotherapy}

Bim expression was found to be lost in a large part of renal cell carcinoma (RCC) [539]. Apoptosis sensitivity correlated with Bim protein levels [539]. Inhibition of histone deacetylation restored Bim expression in RCC cell lines [539]. Bim expression was lower in AJCC II-IV stages of melanoma than in AJCC I-II stages [540]. Loss of PTEN in B-Raf ${ }^{\mathrm{V} 600 \mathrm{E}}$-mutated melanoma cells confers resistance to the B-Raf inhibitor PLX4720 through the suppression of Bim expression [541]. PLX4720 stimulated
Akt activity in PTEN-, but not in $\mathrm{PTEN}^{+}$cells [541]. The treatment resistance of PTEN- cells could be overcome by co-treatment of PLX4720 with a PI3K inhibitor that leads to enhanced Bim expression [541].

G1P3, a survival protein induced by interferons and a contributor to poor outcomes in estrogen receptor (ER)-positive breast cancer patients, attenuated the induction of Bim [542]. Elevated expression of G1P3 was associated with decreased relapse-free and overall survival in ER-positive breast cancer patients [542]. Abnormal overexpression of the chaperone-associated E3-ligase $\mathrm{C}$ terminus of Hsc70-interacting protein (CHIP) induced apoptosis resistance in breast cancer cells by activating the Akt pathway with subsequent prevention of FoxO-dependent Bim and PTEN transcription [543]. CHIP activates the Akt pathway through targeting PTEN for proteasomal degradation [543, 544]. Pyruvate kinase M2 (PKM2) overexpressed in hepatocellular carcinoma promotes Bim degradation and is associated with poor outcome [545]. Depletion of PKM2 induced apoptosis that could be prevented by simultaneous silencing of Bim [545].

Bim expression was low or intermediate in $64 \%$ of EGFR mutation-positive NSCLC and high in 36\% of the patients. Those with higher Bim expression showed longer progression-free survival when treated with the EGFR tyrosine kinase inhibitor erlotinib and longer overall survival [546-548]. EGFR signaling prevents Bim expression through activation of the MAPK-ERK and PI3K-Akt signaling pathways. Imatinib that inhibits c-Kit activity in gastrointestinal stromal tumors, leads to an increase in the dephosphorylated and deubiquitinated form of Bim in addition to increased FoxO3a-induced Bim transcription [549].

Overexpression of the differentiation-related gene1 (Drg1) promotes metastasis of colorectal cancer and confers resistance to the topoisomerase inhibitor irinotecan (CPT-11) [550]. Drg1 interacts with Bim and negatively regulates its stability by promoting its interaction with the ElonginB-Cullin2-CIS ubiquitin-protein ligase complex resulting in proteasomal degradation [550]. In the absence of Drg1, Bim was stabilized and bound more abundantly to Hsp70, thereby sensitizing the tumor cells to irinotecan [550]. Similarily, the Receptor for activated C kinase 1 (RACK1) has been shown to confer paclitaxel resistance to breast cancer cells by binding to both the dynein light chain 1 and Bim $_{\mathrm{EL}}$ upon exposure to paclitaxel [551]. RACK1 then promotes the degradation of $\mathrm{Bim}_{\mathrm{EL}}$ through the ElonginB/C-Cullin2-CIS complex [551]. RACK1 is frequently overexpressed in solid tumors and the CIS protein level was negatively correlated with $\mathrm{Bim}_{\mathrm{EL}}$ in cancer specimens [551].

\subsubsection{Role of Bim in TGF $\beta$-induced apoptosis}

TGF $\beta$ is a pleiotropic cytokine that can induce various signal transduction pathways, ultimately leading 
to cell growth, apoptosis or tumor progression, dependent on the cellular context [552] (Figure 10). TGF $\beta$ induces apoptosis of normal epithelial cells, osteoclasts and lymphoid cells by a mechanism that depends on Bim and Bmf [42, 152, 153, 166, 553-555]. Acquisition of resistance to TGF $\beta$-induced apoptosis is a critical step for carcinogenesis in many organs.

The transcription factors $\operatorname{Smad} 3 / 4$ and Runx1/3, the p38 MAPK signaling pathway, and the generation of ROS are all involved in the TGF $\beta$-mediated upregulation of Bim transcription [152, 164, 553]. Runx1 cooperates with FoxO3 to transcriptionally induce Bim [165]. TGF $\beta$ also induces the expression of the MAPK phosphatase MKP2/DUSP4 to rapidly increase Bim $_{\mathrm{EL}}$ by inactivation of ERK1/2 [166]. The Birt-Hogg-Dubé (BHD) tumor suppressor gene was found to be important for TGF $\beta$ induced Bim expression [556]. Loss-of-function mutations in BHD lead to increased risk for skin and kidney cancer [556].

TGF $\beta$ could sensitize gastric carcinoma cells to TNF $\alpha$-induced apoptosis where TGF $\beta$ is responsible for Smad3-induced Bim transcription and $\mathrm{TNF} \alpha$ to JNK-mediated stabilization of the Bim protein [153].
The tyrosine kinase inhibitor sorafenib could sensitize hepatocellular carcinoma cells to TGF $\beta$ through Bim upregulation and Mcl-1 downregulation [557]. TGF $\beta$ could also sensitize non-small cell lung carcinomas to the broad-acting tyrosine kinase inhibitor dasatinib through upregulation of Bim and downregulation of Smad7 [558]. Smad7 is a negative regulator of the TGF $\beta$ signaling pathway that inhibits Bim expression [42]. miR$106 \mathrm{~b} \sim 25$ that targets Bim, also targets Smad7, resulting in activation of TGF $\beta$ signaling and induction of EMT in human breast cancer [559]. Thus, miR-106b 25 promotes tumor progression by a dual mechanism. The homeotic transcription factor Six1, which is involved in breast cancer progression and metastasis through upregulation of miR-106b 25, switches the TGF $\beta$ signaling from being tumor suppressive to tumor promotional [559]. The miR$106 \mathrm{~b} \sim 25$ cluster is also upregulated by E2F1 that leads to TGF $\beta$-resistance in gastric cancer [175].

Early stage intestinal adenomas with mutations in the adenomatous polyposis coli (APC) gene were more sensitive to TGF $\beta$-induced apoptosis than wild-type intestinal crypts [554]. This was explained by higher Bim levels in the APC mutant cells, and IGF-R signaling in

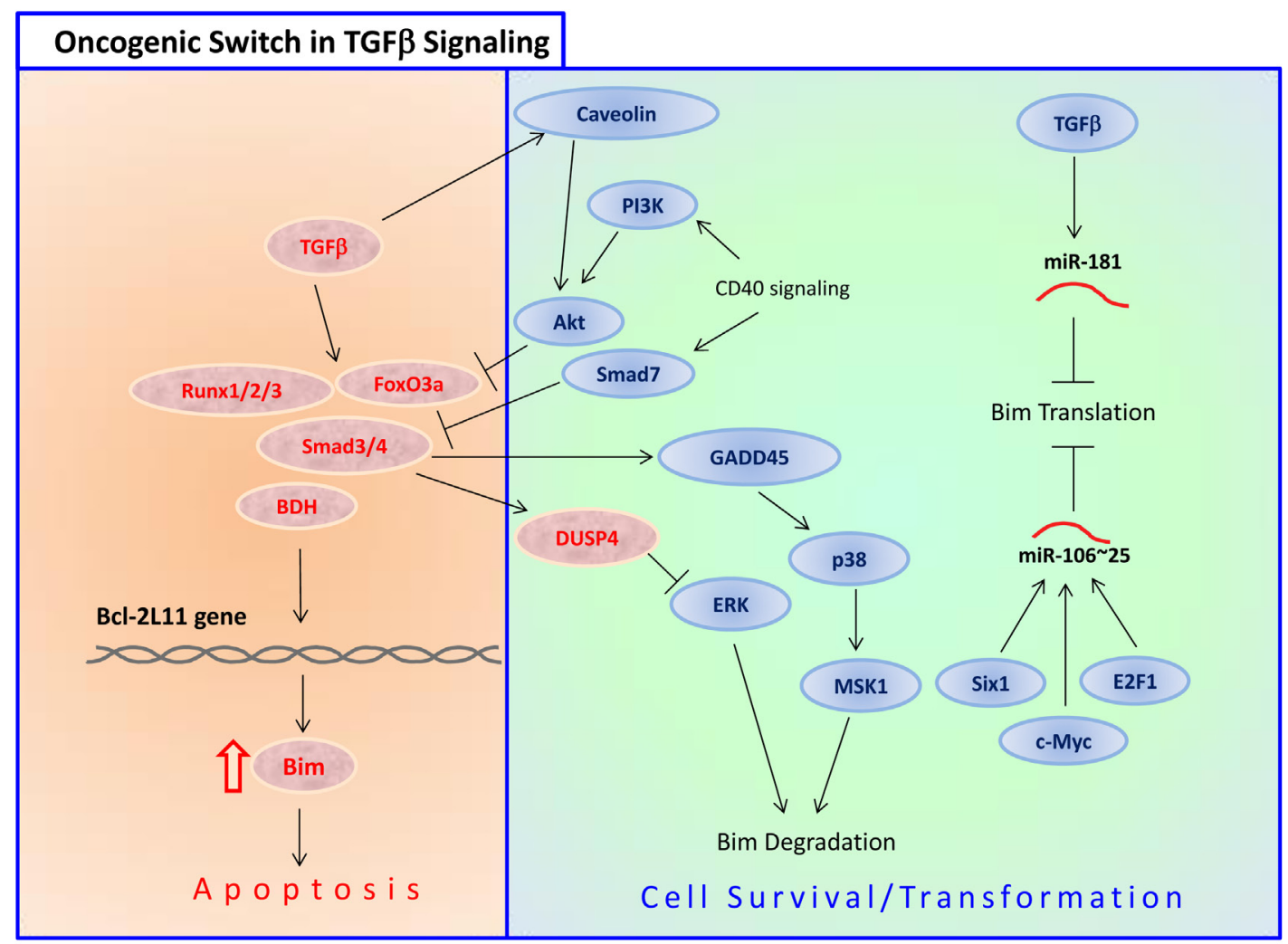

Figure 10: The decision between TGF $\beta$-induced apoptosis and cell transformation. TGF $\beta$ may induce either apoptosis or cell transformation dependent on the cellular context (Section 4.11.5.1). TGF $\beta$ induces Bim expression by activating the Runx1/2/3, Smad3/4 and FoxO3a transcription factors. Also, BDH was found to be important for Bim induction. The simultaneous induction of DUSP4 that antagonizes ERK signaling, leads to stabilization of the Bim protein. TGF $\beta$ may also promote cell survival through Akt and MSK1dependent mechanisms. Upon oncogenic switch, Bim expression is downregulated by reduced transcription, increased degradation and inhibition of Bim translation by the oncomiRs miR-106 25 and miR-181. miR-106 25 is upregulated by E2F1, c-Myc and Six1, while TGF $\beta$ itself may upregulate miR-181. 
wild-type cells that antagonizes Bim [554]. Mutant K-Ras oncogene activation led to TGF $\beta$ resistance in APC-mutant cells due to activation of ERK1/2 [554]. The K-Ras mutant cells were still sensitive to $\mathrm{BH} 3$ mimetics, suggesting that the resistance is due to impaired Bim upregulation and not defects in downstream pathways [554].

In cultured hepatocytes, TGF $\beta$ rapidly activates Akt through a mechanism dependent on Caveolin-1, leading to suppression of Bim expression and apoptosis [560]. In highly metastatic breast cancer cells, autocrine TGF $\beta$ protects the cells from apoptosis through suppression of Bim [561]. In these cells, TGF $\beta$ suppressed the forkhead box $\mathrm{C} 1$ (FoxC1) required for Bim expression [561]. TGF $\beta$ may also promote breast cancer metastasis through upregulation of miR-181a that targets Bim [262]. Moreover, TGF $\beta$ activates mitogen- and stress-activated protein kinase 1 (MSK1) that prevents TGF $\beta$-induced apoptosis through downregulation of Bim [562]. Induction of MSK1 activity by TGF $\beta$ depends on Smad4-induced GADD45 expression, which in turn activates p38 MAPK that positively regulates MSK1 [562]. These activities of TGF $\beta$ may explain its dual actions on tumorigensis.

\section{THERAPEUTIC APPLICATIONS}

Under normal conditions, Bim expression is tightly and timely regulated. However, excessive Bim activation may lead to neurodegenerative diseases, liver damage and diabetes, while reduced Bim expression is associated with autoimmune diseases, increased risk for cancer development and poor response of malignant cells to chemotherapeutics (Figure 8). One strategy to treat these conditions is through regulating Bim expression and/or activity.

In Parkinson's disease for instance, excessive ROS production due to mitochondrial Complex I dysfunction is responsible for JNK-mediated activation of Bim that leads to degeneration of dopaminergic neurons of the substantia nigra pars compacta (SNpc) [445]. Inhibition of JNK or neutralizing ROS by using anti-oxidants should help to attenuate disease progression. This may also be applicable for diabetes that is also caused by ROS-induced JNKdependent Bim activation [188]. Osteocalcin reduces high glucose-induced $\mathrm{H}_{2} \mathrm{O}_{2}$ levels and protects $\beta$-cells from apoptosis [563].

As Mst1 leads to $\mathrm{Pdx}-1$ degradation and Bim upregulation [459], inhibition of Mst1 may also prevent $\beta$-cell dysfunction. Mst1 is activated by ROS and promotes apoptosis through phosphorylation of and activation of FoxO transcription factors [564]. Mst1 activity is inhibited by interaction with the redox sensor thioredoxin-1 (Trx1) [564], whose activity, in turn, is antagonized by Thioredoxin-interacting protein (Txnip). Txnip is upregulated by high glucose and dexamethasone in insulin-producing $\beta$-cells, leading to apoptosis of these cells $[565,566]$. Incretins such as GLP-1 and exendin-4, protect against $\beta$-cell damage [567]. GLP-1 has been shown also to diminish neuronal degeneration and death caused by NGF deprivation through suppressing Bim induction [369]. Thus, GLP-1 and exendin-4 are not only beneficial for the treatment of type 2 diabetes, but also for neurodegenerative disorders such as Alzheimer's and Parkinson's diseases [568, 569]. Incretins could also delay the onset of diabetes and neurodegeneration that appear in Friedriech's ataxia disorder caused by Frataxin deficiency [570].

Untimely activation of Bim in activated specified $\mathrm{T}$ cells may lead to premature resolution of the immune response, resulting in persistent infection, chronic inflammation and cancer development. Increasing the survival of viral-specific or tumor-specific T cells, e.g., by preventing PD-1 signaling or increasing 4-1BB (CD137) signaling [410], should lead to increased anti-viral and anti-tumor immune responses. On the other side of the Bim coin, too little Bim expression in hematopoietic cells may lead to skewed immune responses.

Chemoresistance in cancer cells is often due to improper Bim function that might be due to disturbance of one or more of the regulatory pathways described in this review. These include reduced transcription due to excessive PI3K-Akt activation, hyperexpression of transcriptional repressors (e.g., YY1) and/or epigenetic silencing; altered Bim splicing; reduced translation due to elevated expression of microRNAs or RNA-binding proteins; reduced protein stability due to hyperactivation of the Ras-MEK-ERK pathway; and Bim sequestration to anti-apoptotic proteins among them overexpression of Mcl-1 is a critical determinant. Different chemotherapeutic interventions have been developed to restore Bim activity that act either upstream or downstream to the critical regulatory point. These include suppression of the inhibitory signal transduction pathway, prevention of Mcl1 expression and use of $\mathrm{BH} 3$ mimetics. These approaches will be described in more details below.

\subsection{Use of BH3 Mimetics as Anti-Cancer Agents}

Compounds that mimic the $\mathrm{BH} 3$ domain of Bim may disrupt the interaction of $\mathrm{BH} 3$-containing proteins to antiapoptotic $\mathrm{Bcl}-2$ proteins, thus releasing $\mathrm{BH} 3$-containing proteins to initiate intrinsic apoptosis. These drugs will only be efficient against tumors that show dependency on anti-apoptotic proteins. ABT-737 is a $\mathrm{BH} 3$ mimetic that binds with high affinity to and antagonizes the functions of Bcl-2 and Bcl-xL, but not Mcl-1 [571]. ABT-737 displaces Bim from the $\mathrm{BH} 3$-binding pocket of Bcl-2, allowing Bim to activate Bax and induce mitochondrial outer membrane permeabilization (MOMP) [515]. The amount of Bim sequestrated to $\mathrm{Bcl}-2$, rather than total $\mathrm{Bcl}-2$ expression levels, determines cellular sensitivity to ABT-737 [309, 515, 572]. An increase in Bim expression, e.g., by using the HDAC inhibitor suberoyl bis-hydroxamic acid 
(SBHA), potentiates the effect of ABT-737 [573].

Preclinical studies demonstrated that ABT-737 induces apoptosis and potentiates the anti-tumor activity of multiple agents in various cancers, including leukemia [571]. The BH3 mimetic ABT-737 increased the response to EGFR tyrosine kinase inhibitors [31]. But since ABT737 antagonizes Bcl-2 and Bcl-xL, but not Mcl-1, which is overexpressed, among others in NSCLC [574], the effect of this $\mathrm{BH} 3$ mimetic is limited, unless combined with a drug reducing Mcl-1 expression. The dual PI3K/mTOR inhibitor NVP-BEZ235 that decreases Mcl-1 expression, synergized with ABT-737 in inducing apoptosis of ovarian carcinoma cells, provided that Bim expression was induced [575]. Simultaneous inhibition of the ERK1/2 pathway restored Bim expression and sensitized low Bim-expressing cancer cells to NVP-BEZ235/ABT-737 treatment [575]. Also Rahmani et al. [576] observed that concomitant inhibition of the PI3K/Akt/mTOR pathway and Bcl-2 using BEZ235 and ABT-737 increased apoptosis in human myeloid leukemia cells. This treatment led to reduced Mcl-1 expression, GSK3 activation, release of Bim from Bcl-2/Bcl-xL and release of Bak and Bax from Mcl-1/Bcl-2/Bcl-xL [576]. Treatment of high-grade serous ovarian cancer ex vivo with ABT-737 and carboplatin, which can indirectly inhibit Mcl-1, showed a requirement for Bim and low activity of ERK to obtain a response to ABT-737 [577]. In the E $\mu$-Myc mouse model, caloric restriction reduced Mcl-1 expression through inhibition of protein translation and sensitized E $\mu$-Myc lymphoma cells to ABT-737 treatment [578].

Although Mcl-1 dominance renders squamous cell carcinoma cells resistant to ABT-737, the HDAC inhibitor vorinostat primes them for sensitivity to ABT-737 by shuttling Bim from Mcl-1 to Bcl-2/Bcl-xL, resulting in synergy for this drug combination and sustained tumor regression in vivo [579]. Somatic FBW7 mutation in squamous cell carcinoma cells is associated with stabilized Mcl-1 and high Bim levels, resulting in a poor response to standard chemotherapy, but a robust response to HDAC inhibitors and enhanced synergy with the combination vorinostat/ABT-737 [579]. Also, combined treatment of ABT-737 with a HDAC inhibitor could overcome adaptive bortezomib resistance of multiple myeloma cells through re-expression of Bim [507]. Chloroquine that disrupts autophagy, further enhanced HDACi/ABT-737 lethality in these cells [507].

ABT-263 (Navitoclax) is a clinical derivative of ABT-737 that are currently undergoing phase I and II clinical evaluation in various tumor types including leukemia [580-582]. In vivo, Navitoclax induced durable and complete tumor regression in a murine xenograft model of acute lymphocytic leukemia and significantly improved the cure rate of rituximab (anti-CD20 mAb) plus chemotherapy in a xenograft model of mantle cell lymphoma [583]. Small-cell lung cancer (SCLC) with high Bim expression is among the most sensitive cells to ABT-263 treatment [584]. As with ABT-737, ABT263 activity is directed against Bcl-2 and Bcl-xL, but not Mcl-1, making Mcl-1 expressing cells treatment resistant. Simultaneous treatment with a TORC1/2 inhibitor (e.g., AZD8055) that reduces Mcl-1 expression improved the response of SCLC to ABT-263 [584].

Alford et al. [585] also showed that primary B-ALL cells expressing high levels of Bcl-2 exhibited great sensitivity to ABT-263 and ABT-199. ABT-263 disrupted Bcl-2:Bim interaction in the cells [585]. However, overexpression of Mcl-1 rendered the B-ALL cells resistant to ABT-263 and ABT-199 [585]. The Mcl1 and Bcl-xL-dependent resistance to ABT-199 could be overcome by inhibiting the PI3K/Akt/mTOR pathway in lymphoid malignancies [586]. NVP-BEZ235, a dual inhibitor of AKT and mTOR, reduced Mcl-1 levels causing Bim release from $\mathrm{Mcl}-1$ and $\mathrm{Bcl}-\mathrm{xL}$, thus leading to cell death by Bax activation [586]. The PI3K $\delta$ inhibitor GS-1101 (idelalisib) downregulated Mcl-1 and sensitized resistant cells to ABT-199 [586].

Obatoclax (GX15-070) binds to Mcl-1 and A1, besides Bcl-2, Bcl-xL and Bcl-w, albeit at low affinity [587]. Obatoclax also kills Bak/Bax deficient cells, thus having additional effects in addition to be a BH3 mimetic [588]. It leads to the upregulation of Noxa that promotes the dissociation of Bak from Mcl-1 [589]. Obatoclax also induces autophagy and necroptosis [590]. Obatoclax is not efficient as a single agent to treat cancer and may cause neurological toxicity that limits its use. Obatoclax may increase the susceptibility of multiple myeloma cells to other chemotherapeutic drugs such as melphalan, dexamethasone and bortezomib [591]. Other Mcl-1 inhibiting agents such as gossypol and its synthetic analogue AT-101 could sensitize otherwise glucocorticoidresistant MLL-arranged ALL cells to glucocorticoidinduced apoptosis [592]. These authors showed that gossypol and AT-101 increased Bim expression and the apoptosis could proceed without downregulation of Mcl-1 [592].

A series of indole-2-carboxylic acid derivatives have been formulated to target Mcl-1 [593]. One of these compounds, A-1210477, induces apoptosis and kills multiple myeloma and non-small cell lung cancer cell lines [593]. A-1210477 disrupts the Mcl-1:Bim and Mcl1 :Noxa complexes, while having no effect on Bcl-2:Bim interactions [593]. A-1210477 synergized with the Bcl-2/ Bcl-xL inhibitor Navitoclax to kill a variety of cancer cell lines [593]. The pan-Bcl-2 inhibitor (-)B197D6 induced apoptosis of acute myeloid leukemia (AML) cells by disrupting Mcl-1/Bim and Bcl-2/Bax interactions [594].

Kazi et al. [595] developed a BH3 $\alpha$-helical mimetic BH3-M6 that binds to Bcl-xL, Bcl-2 and Mcl1 , thereby preventing their binding to $\mathrm{Bax}, \mathrm{Bak}, \mathrm{Bad}$ and Bim. BH3-M6 disruption of these protein-protein interactions is associated with cytochrome $\mathrm{C}$ release from mitochondria, caspase 3 activation and Poly(ADP-ribose) 
polymerase (PARP) cleavage [595]. BH3-M6 sensitized tumor cells to a proteasome inhibitor [595]. LaBelle et al. [596] developed a hydrocarbon-stapled peptide of the Bim BH3 helix that targeted Bcl-2 family members with high affinity, leading to cell death of otherwise resistant hematologic malignancies. The stapled peptide is structurally stable, protease-resistant and cell-permeable. The stapled Bim BH3 helix (amino acids 146-166) contains an i, i+4 all-hydrocarbon crosslink spanning positions 154 and 158 [596].

\subsection{Use of Protein Kinase Inhibitors to Increase Bim Expression and Activity}

The use of $\mathrm{BH} 3$ mimetics is hampered by high hepatotoxicity, which limits their applications. Also, the dependency on sufficient Bim expression for apoptosis induction makes $\mathrm{BH} 3$ mimetics unsuitable for overcoming tumor resistance caused by low or absent Bim expression [577]. In many cases, Bim expression is repressed through activation of the PI3K-Akt-mTOR and/or Ras-MEKERK1/2 signaling pathways, and inhibition of these pathways might be sufficient for Bim upregulation and induction of apoptosis. Usually combined treatment of drugs targeting different pathways is advantageous.

The mTOR inhibitors rapamycin (Sirolimus) and RAD001 (Everolimus) have long been known to increase the sensitivity of malignant hematopoietic cells to chemotherapeutic drugs such as glucocorticoids [519, 533, 534, 597-599], rituximab [600] and IFN $\alpha$ [601], and solid tumors to cisplatin [602], mitoxantrone [603], carboplatin [604], vinorelbine [604] and taxoids $[603,604]$. One of the mechanisms by which rapamycin sensitizes tumor cells is through downregulation of Mcl1 with simultaneous upregulation of Bim [533, 534]. Inhibition of glucose metabolism or mTORC1 leads to decreased Mcl-1 expression and upregulation of Bim, thereby sensitizing diffuse large B cell leukemic cells to ABT-737 [605]. As rapamycin inhibits TORC1, but not TORC2, what leads to a feedback loop activating the Akt pathway [606] and further Mcl-1 stabilization [108], dual TORC1/TORC2 inhibitors have been developed with enhanced synergistic effect on other chemotherapeutics. In the case of rapamycin sensitization of oral squamous cell carcinoma cells to cisplatin, rapamycin increased FoxO3a protein stability and cisplatin inhibited the feedback activation of Akt by rapamycin [602]. This resulted in FoxO3a activation and Bim induction [602]. Similarily, the Akt inhibitor MK-2206 enhanced the anti-tumor effect of rapamycin on neuroblastoma cells [607]. The dual TORC1/2 inhibitor AZD8055 sensitized colorectal cancers with K-Ras or B-Raf mutations to ABT-263 by suppressing Mcl-1 [608]. AZD8055 induced apoptosis in laryngeal carcinoma by upregulating Bid, Bad and Bim [609]. The dual mTORC1/TORC2 inhibitor
OSI-027 induced apoptosis in specimens from B cell acute lymphoblastic leukemia, mantle cell lymphoma and marginal zone lymphoma by a mechanism that was dependent on Puma and Bim [610]. The PI3K/mTOR dual inhibitor NVP-BEZ235 reduced Mcl-1 expression and sensitized ovarian carcinoma cells to ABT-737 through a Bim-dependent mechanism [575]. Inhibition of the PI3K/ Akt pathway also sensitized lymphoid malignancies to glucocorticoid-induced apoptosis, which can be explained by the requirement for both GSK3 and Bim [63].

Sorafenib, a potent multikinase inhibitor, induces apoptosis of human acute myeloid leukemia (AML) cells through downregulating $\mathrm{Mcl}-1$ and enhancing binding of Bim to Bcl-2 and Bcl-xL [611]. Sorafenib may also upregulate Bim expression [612]. Combined treatment of sorafenib with obatoclax or ABT-737 had a synergistic effect in reducing tumor growth [611, 612]. Similarly, combining obatoclax with the pan-CDK inhibitor flavopiridol increased apoptosis of both drug-naïve and drug-resistant multiple myeloma cells in a Bim- and Noxadependent mechanism [613]. Flavopiridol inhibited Mcl-1 transcription, but increased transcription of Bim and its binding to Bcl-2 and Bcl-xL [613]. Obatoclax prevented Mcl-1 recovery and caused release of Bim from Bcl-2, Bcl-xL and Mcl-1, accompanied by activation of Bak and Bax [613].

Simultaneous targeting of PI3K and mTOR using NVP-BGT226 induced apoptosis in multiple myeloma cells by upregulating Bim [614]. The growth stimulatory effect of IGF1 and IL-6 on multiple myeloma cells was completely abrogated by NPV-BGT226 [614]. NVPBGT226 has also been shown to exert cytotoxic effects against other cancer cell types such as ALK-positive anaplastic large cell lymphoma [615] and hepatocellular carcinoma [616] and has entered Phase I/II clinical trials for breast cancer [617].

Inhibition of EGFR using the tyrosine kinase inhibitors (TKIs) erlotinib or gefitinib could induce apoptosis of NSCLC with mutant constitutively active EGFR ( $\triangle \mathrm{L} 747-\mathrm{S} 752$ or L858R) through a process dependent on Bim [306, 307, 309]. The three Bim isoforms $\mathrm{Bim}_{\mathrm{EL}}, \mathrm{Bim}_{\mathrm{L}}$ and $\mathrm{Bim}_{\mathrm{S}}$ were induced by erlotinib in single mutated EGFR-sensitive cells, but not in PTENdeficient or double mutated EGFR (additional T790M mutation)-insensitive cells [307, 308, 546]. Pretreatment mRNA levels of Bim predicted the capacity of EGFR inhibitors to induce apoptosis in EGFR-mutant cancer cells [618]. TKI-resistance usually develops upon repeated treatment which was shown in some cases to be due to overexpression of paxillin [619] or neutrophil gelatinase-associated lipocalin (NGAL) [620]. Both paxillin and NGAL activate ERK, resulting in enhanced Bim degradation and increased Mcl-1 expression [619, 620]. Patients with lower plasma NGAL levels showed a better erlotinib response [620]. TKI-mediated reactivation of ERK1/2 is also due to reduced Akt-dependent Ets- 
1-mediated DUSP6 induction [621]. TKI resistance could be overcome by simultaneous delivery of an ERK inhibitor (AZD6244) [619]. Also, the HDAC inhibitor vorinostat could circumvent EGFR-TKI resistance in EGFR-mutant NSCLC cells [32]. Another mechanism for acquired resistance to erlotinib or gefitinib is the appearance of a secondary EGFR mutation. A secondgeneration TKI afatinib binds to the mutated EGFR, but causes redistribution of EGFR to the cell surface through a Rab11a-dependent recycling that reduces its effectiveness [622]. However, combining afatinib with the anti-EGFR monoclonal antibody cetuximab synergistically induced apoptosis of the erythroleukemic K562 cells through upregulation of Bim [622].

Hepatocyte growth factor (HGF) confers EGFR TKI resistance by inducing two cancer-promoting functions. HGF makes the cancer cells independent of EGFR signaling and enables EGFR to interact with other proteins such as CUB domain-containing protein-1 (CDCP1), EphA2 and AXL, forming a c-Met (HGFR)EGFR cross-talk that can't be inhibited by EGFR TKI treatment [546]. The dual anaplastic lymphoma kinase (ALK) and c-Met receptor tyrosine kinase inhibitor CM118 inhibited ALK-signaling and HGF-induced c-Met signaling, thus interrupting the c-Met-EGFR crosstalk. This compound led to inhibition of proliferation or induction of apoptosis in c-Met- and ALK-addicted cancer cells [623]. Combined with EGFR inhibitors, CM118 induced apoptosis of c-Met amplified NSCLC cells through Bim upregulation and Mcl-1 downregulation [623]. mTOR inhibitors further potentiated the antitumor effect of CM-118 [623]. Also, the dual ALK/cMet inhibitor crizotinib that is effective in treating EML4-ALK positive and c-Met amplificated NSCLC patients [624], induces apoptosis by a Bim-dependent mechanism [625]. Bim upregulation by ALK inhibition was a result of ERK suppression [626]. Crizotinib also induced apoptosis of c-Met-positive gastric cancer cells through upregulation of Bim [627]. Foretinib, which is an oral multikinase inhibitor that inhibits c-Met, Recepteur d'origine nantais (RON; MST1R), the Gas6 tyrosine kinase receptor AXL and vascular endothelial growth factor receptor (VEGFR), improves overall survival in a preclinical model of hepatocellular carcinoma, through upregulation of Bim and p27 and downregulation of cyclin B1 and phosphorylated c-Myc [628]. Overexpression of AXL in CML led to imatinib resistance that was related to increased $\mathrm{PKC} \alpha / \beta$ and ERK1/2 activation [629]. The $\mathrm{AXL}$ receptor tyrosine kinase receptor is also involved in the survival of B-CLL cells. Combining the AXL inhibitor TP-0903 with Bruton's tyrosine kinase inhibitors (e.g., ibrutinib) reduced the expression of Mcl-1, Bcl-2 and XIAP, while upregulated Bim expression, resulting in increased B-CLL apoptosis [630].

Treatment of B-Raf ${ }^{\mathrm{v} 600 \mathrm{E}} / \mathrm{PTEN}$-null melanoma cells with the B-Raf inhibitor vemurafenib led to increased fibronectin expression that abrogates the therapeutic response due to enhanced PI3K-Akt signaling and Mcl-1 expression [631]. Simultaneous use of a PI3K inhibitor overcomes the drug resistance [631]. Acquired B-Raf inhibitor resistance in melanoma cells could also be overcome by simultaneous use of the ATP-competitive MEK/Aurora kinase inhibitor BI-847325 [632]. BI847325 decreased the expression of MEK and Mcl-1, while increased the expression of Bim [632]. Combining vemurafenib with the MEK inhibitor trametinib increased Bim upregulation and apoptosis [633]. Trametinib alone caused activation of Akt in B-Raf non-V600 mutated cells that was nullified with the combination of vemurafenib [633]. Another approach to overcome vemurafenib resistance is to use the Hsp90 inhibitor XL888 [634] or ganetespib [635]. XL888 induced apoptosis of vemurafenib-resistant melanoma cells by increasing FoxO3a-induced Bim transcription and downregulation of Mcl-1 [634].

Altogether, these studies repeatedly show that Bim function can be revived in cancer cells, and the most efficient treatment is usually a drug combination that simultaneously targets various critical nodal points ultimately leading to Bim upregulation (e.g., PI3K-Akt inhibitors, HDAC inhibitors), Bim stabilization (e.g., ERK inhibitors, proteasomal inhibition), and Bim release from sequestered intracellular storage (e.g., BH3 mimetics, JNK activation, dual TORC1/2 inhibitors).

\subsection{The Bim Status as a Prognostic Criterium}

Usually cancer cells with high basal Bim expression show better response to Bim-dependent chemotherapy than those with low Bim expression, which can be explained by the rapid available pre-made Bim, a state termed "primed for apoptosis". When Bim is expressed at relative high basal levels, the cancer cells have often developed a mechanism (e.g., concomitant Mcl-1 or Bcl-2 upregulation) that antagonizes the pro-apoptotic function of Bim. As the cancer cells become dependent on the anti-Bim mechanism for survival, targeting this mechanism will induce Bim-dependent cancer cell death. For instance, it was sufficient to reduce Mcl-1 levels to induce Bim-dependent apoptosis in c-Myc positive HERpositive breast cancer cells [192]. Not only the basal Bim level account for better prognosis, but also the ability of the cancer cells to elevate Bim expression in response to chemotherapy is important for the clinical response. This has been demonstrated for glucocorticoid susceptibility of pediatric acute lymphoblastic leukemia (ALL) [521, $522,636]$. Bim was only upregulated in prednisolone/ dexamethasone-sensitive pediatric ALL cells, but not in those being resistant $[521,636]$. Patients whose Bim protein expression levels failed to upregulate at day 8 compared to day 0 had a poorer event-free survival than those patients whose Bim expression levels did upregulate 
[521]. The resistance was correlated with reduced histone $\mathrm{H} 3$ acetylation and could be overcome by the HDAC inhibitor vorinostat [522]. Also, Bim induction in chronic lymphocytic leukemia in response to glucocorticoids correlated with the response rate [637].

The pretreatment RNA levels of Bim predicted the ability of EGFR, HER2 and PI3K inhibitors to induce apoptosis in EGFR-mutant, HER2-amplified and PIK3CAmutant cancer cells, respectively, while Bim levels did not predict responsiveness to standard chemotherapies such as gemcitabine and cisplatin [618]. Also, the extent of Bim induction in response to the inhibitors is predicative [618]. Patients with EGFR-mutant lung cancers expressing high levels of Bim showed longer progression-free survival (PFS) than those with tumors expressing low levels of Bim [618]. PFS to erlotinib was longer for high Bim-expressing NSCLC than those with intermediate or low Bim expression [548]. Bim RNA levels may be assessed in diagnostic cancer specimens to predict which patients will benefit from single-agent kinase inhibitor [618]. Glioblastoma multiforme patients with high levels of phospho-Bad (Ser136) and phospho-Bim (Ser69) indicative for increased ERK activation displayed shorter overall survival [638]. IgM-induced phosphorylation of Bim correlated with progressive disease in CLL patients with mutated IGHV genes [511].

Bim deletion polymorphism was significantly associated with the clinical efficacy of tyrosine kinase inhibitors in terms of response rate and disease control rate in EGFR-mutated NSCLC patients, but not in CML or hepatocellular carcinoma (HCC) [639]. EGFR-mutated NSCLC patients harboring Bim deletion polymorphism was associated with a shorter PFS, while there was no association with overall survival [639]. Shorter overall survival of pediatric acute lymphoblastic leukemia patients was associated with the Bim C29201T polymorphism located in the $\mathrm{BH} 3$ domain [34].

A strategy termed $\mathrm{BH} 3$ profiling using $\mathrm{BH} 3$ peptides can identify apoptotic defects in cancer cells. $\mathrm{BH} 3$ profiling of CLL can identify cells that require $\mathrm{Bcl}-2$ for survival and can predict sensitivity to ABT-737 [515, 572]. Bcl-2 dependence correlates with high levels of Bim sequestered by Bcl-2 [572].

\section{CONCLUSIONS}

In this review we have attempted to survey the various signal transduction pathways regulating the expression and activity of Bim. The multiple regulatory mechanisms affecting Bim, make it critical for determining the cell fate in response to any changes in the cell's microenvironment or in the intrinsic cell signal pathways. Many of these pathways can also affect Mcl-1 expression, often in an inverse manner than Bim, thereby tipping the Bim/Mcl-1 balance towards either apoptosis or survival. As an essential component of the intrinsic apoptotic pathway, alterations in Bim expression affect almost every process in the body from regulating the immune system, the nerve system, $\beta$-cell and liver physiology to affecting mammary lumen formation and cancer progression. Therefore there is no wonder why abnormal Bim expression causes a range of pathological conditions (Figure 8). As Bim expression is regulated differentially in different cell types, each pathological condition needs to be considered separately. For instance, an increase in intracellular cAMP levels makes malignant lymphoid cells more susceptible to an apoptotic stimulus, while in insulin-producing $\beta$-cells and neurons it is protective. Another example of a cell-specific effect is JNK activation. In neuronal cells and $\beta$-cells excessive JNK activation is detrimental, activating Bim-dependent cell death, while in lymphoid cells, JNK activation actually antagonizes apoptosis. This cell-specific effect may be explained by Bim sequestration to microtubules in the former cell types where JNK phosphorylates and releases Bim, while in lymphoid cells pre-made Bim is tonically sequestered to anti-apoptotic proteins rather than microtubules. Death of various immune cells can be prevented by respective cytokines. For instance, IL-7 can rescue T cell death, whereas IL-4 and BAFF prevent $B$ cell death. In the context of cancer, increased survival of tumor-specific cytotoxic $\mathrm{T}$ cells can be achieved by interrupting the PD-1L/PD-1 or enhancing the $4-1 \mathrm{BB}$ signaling using specific antibodies, thereby increasing the anti-tumor effect [410]. On the contrary, in autoimmune diseases, elimination of autoreactive $\mathrm{T}$ and $\mathrm{B}$ cells is desirable. Several approaches have been examined that can increase apoptosis of autoreactive immune cells. These include the use of ABT-737 mimetics [640], monoclonal antibody against IL-6R (e.g., tocilizumab or siltiximab) [641, 642], mTOR inhibition [643] or activation of the PD-1 pathway [644], all tipping the Bim/Mcl-1 balance in favor of Bim. Another approach is to prevent the death of target cells affected by the autoimmune cells. This is best exemplified by the intrinsic higher $\beta$-cell death in diabetes-predisposed patients that triggers insulitis which, in turn, promotes further $\beta$-cell death, thereby forming a bad negative feedback loop. As we have learned, some of the diabetes susceptibility genes e.g., GLIS3, PTPN2, $\mathrm{BACH} 2$ and Cathepsin $\mathrm{H}$, regulate Bim expression. Thus, preventing Bim upregulation in $\beta$-cells should reduce the propensity to develop diabetes. Similarly, preventing $\beta$-amyloid aggregation should restrain the development of Alzheimer's disease.

On the other side of the pathological spectrum lies the cancer issue. Each tumor cell has often developed its own characteristic dependency on specific protein kinases that antagonizes Bim expression, while fortifying Mcl-1 expression. Enormous efforts have been made to develop drugs that specifically target the specific upstream kinase responsible for tumor cell survival. Classical examples are the Bcr-Abl/c-Kit inhibitor imatinib, the EGFR inhibitors 
erlotinib and gefitinib, the Flt3 inhibitor ponatinib and the B-Raf ${ }^{\mathrm{V} 600 \mathrm{E}}$ inhibitor vemurafenib, all inducing Bim expression. Although initial clinical response is often seen when using these drugs, adaptive resistance mechanisms frequently develop, leading to cancer recurrence. The main resistance mechanisms involve the single or combined reactivation of the PI3K-Akt-mTOR and Ras-Raf-MEKERK1/2 survival pathways that prevent Bim expression. The current trend is to combine the specific targeting therapies with inhibitors targeting the downstream survival protein kinases. This combined treatment ensures proper reactivation of $\mathrm{Bim}$. Other approaches include the combined treatment of a tyrosine kinase inhibitor with an HDAC inhibitor that aims to synergistically increase Bim expression. In summary, drug-induced apoptosis of tumor cells that depends on the intrinsic apoptotic pathway, needs a sufficient amount of Bim. Any mechanism that prevents Bim expression in cancer cells should therefore be targeted.

\section{HIGHLIGHTS}

- Bim is essential for initiating the intrinsic apoptotic pathway and is often required for efficient response to chemotherapeutics.

- Bim expression and activity are tightly regulated at the transcriptional, translational and post-translational levels, making it readily available when required.

- Protein kinases and phosphatases regulate Bim stability and activity, while microtubules and antiapoptotic proteins of the Bcl-2 family sequester Bim, keeping it in an inactive state.

- Elevated Bim expression leads to neurodegenerative disorders, liver damage and diabetes, while suppression of Bim supports tumor progression and metastasis.

- Therapeutic interventions aim to prevent neuronal, hepatocyte and $\beta$-cell apoptosis by reducing Bim expression, while, on the contrary, should increase Bim expression in cancer cells for apoptosis induction.

- Chronic inflammation prevailing under persistent viral infections and in the tumor microenvironment can be overcome by rescuing cytotoxic $\mathrm{T}$ cells from Bimdependent cell death.

\section{Abbreviations}

ALK - Anaplastic lymphoma kinase

ALL - Acute lymphoblastic leukemia

AML - Acute myeloblastic leukemia

AP-1 - Activator protein-1

APC - Anaphase promoting complex

APRIL - A proliferation-inducing ligand

ARE - AU-rich element

ASK1 - Apoptosis signal-regulating kinase 1
ATF4 - Activating transcription factor 4

ATM - Ataxia telangiectasia-mutated protein kinase

Bak - Pro-apoptotic Bcl-2 antagonist/killer

$\mathrm{BACH} 2$ - Basic leucine zipper transcription factor 2

BAFF - B cell activating factor belonging to the

tumor necrosis factor family

Bax - Bcl-2-associated X

Bcl-2 - B cell lymphoma-2

Bcl-xL - B cell lymphoma-extra large

$\mathrm{BCR}$ - B cell receptor

Bcr-Abl - Breakpoint cluster region-Abelson murine leukaemia viral oncogene

BDH - Birt-Hogg-Dubé

Bfl-1 - Bcl-2-related protein A1

$\mathrm{BH}-\mathrm{Bcl}-2$ homology domain

BHRF-1 - BamHI fragment $\mathrm{H}$ rightward open

reading frame 1 (Epstein-Barr virus homologue of

Bcl-2).

$\mathrm{Bid}$ - BH3 interacting domain death protein

Bim - Bcl-2 interacting mediator of cell death/BOD

(Bcl-2 related ovarian death agonist)

BMCC1 - BNIP2 and Cdc42GAP homology (BCH) motif-containing molecule at the carboxyl-terminal region 1

BNIP2 - Bcl-2/adenovirus E1B 19kDa interacting protein 2

BOD - Bcl-2 related ovarian death agonist

BRCA-1 - Breast cancer susceptibility protein 1

Brd4 - Bromodomain 4

CBP - CREB-binding protein

Cdk4 - Cyclin dependent kinase 4

CHIP - C terminus of Hsc70-interacting protein

CHOP - C/ERB homologous protein

CK2 - Casein kinase II

CLL - Chronic lymphocytic leukemia

CML - Chronic myeloid leukemia

CREB - cAMP response element-binding protein

DBD - Dynein binding domain

E2F - E2 promoter binding factor

EBNA3A/3C - Epstein-Barr virus determined

nuclear antigen $3 \mathrm{~A}$ or $3 \mathrm{C}$

EBV - Epstein-Barr virus

ECM - Extracellular matrix

EGFR - Epidermal growth factor receptor

Egr-1 - Early growth response protein 1

EMT - Epithelial to mesenchymal transition

ER - Endoplasmic reticulum

ERK1/2 - Extracellular signal-regulated kinases 1

and 2

EZH2 - Enhancer of zeste homolog 2

Fbw7 - F-box and WD repeat domain-containing 7

FGF4- Fibroblast growth factor-4

Flt3 - FMS-like tyrosine kinase 3

FoxO3a - Forkhead box O3 isoform a

GADD45a - Growth arrest and DNA-damage-

inducible protein 45 alpha 
GATA-1 - Transcription factor binding to the DNA sequence GATA

GILZ - Glucocorticoid-induced leucine zipper

GLIS - Gli similar

GLP-1 - Glucagon-like peptide-1

GSK3 - Glycogen synthase kinase 3

$\mathrm{H} 2 \mathrm{AX}$ - Histone $2 \mathrm{~A}$ variant $\mathrm{X}$

HCC - Hepatocellular carcinoma

HBV - Hepatitis B virus

$\mathrm{HCV}$ - Hepatitis $\mathrm{C}$ virus

HDAC - Histone deacetylase

HER2 - Human epidermal growth factor receptor 2

HIF-1 - Hypoxia-inducible factor 1

hnRNP C - Heterogeneous nuclear ribonuclear

protein $\mathrm{C}$

HoxB8 - Homeobox protein B8

HGF - Hepatocyte growth factor

Hrk/DP5 - Harakiri protein

Hsc70 - Heat-shock cognate protein 70

Hsp27 - Heat shock protein 27

HSV - Herpes simplex virus

HtrA2 - High temperature requirement serine

protease $\mathrm{A} 2$

IBC - Inflammatory breast cancer

IL-3 - Interleukin-3

IL-7 - Interleukin-7

IP3R - Inositol triphosphate receptor

JAK - Janus-family tyrosine kinase

JNK - c-Jun N-terminal kinase

Keap-1 - Kelch-like ECH-associated protein-1

KLRG1 - Killer-cell lectin like receptor G1

LCMV - Lymphocytic choriomeningitis virus

LPS - Lipopolysaccharide

MAPK-1 - Mitogen-activated protein kinase 1

Mcl-1 - Myeloid cell leukemia-1

MCMV - Mouse cytomegalovirus

MEK - Mitogen-activated protein kinase (MAP)extracellular signal-regulated protein kinase (ERK)

kinase

MKP2/DUSP4 - MAP kinase phosphatase 2/Dual

specificity protein phosphatase 4

MLL - Mixed lineage leukemia

MM - Multiple myeloma

MOMP - Mitochondrial outer membrane

permeabilization

MSK1 - Mitogen- and stress-activated protein

kinase 1

Mst1 - Mammalian sterile-20-like kinase 1

mTOR - Mammalian target of rapamycin

c-Myb - Avian myeloblastosis virus oncogene

cellular homolog

c-Myc - Avian myelocytomatosis virus oncogene

cellular homolog

NGF - Nerve growth factor

NFAT - Nuclear factor of activated T cells

NF $\kappa B$ - Nuclear factor $\kappa B$
NF-Y - Nuclear transcription factor $Y$

NK - Natural killer

NOD - Non-obese diabetes

NSCLC -Non-small-cell lung cancer

PAR - Protease-activated receptor

PARP - Poly(ADP-ribose)polymerase

Pdx-1 - Pancreatic duodenal homeobox 1

Perk - PKR-like endoplasmic reticulum kinase

PHLPP2 - PH domain and leucine-rich repeat

protein phosphatase 2

PI3K - Phosphatidylinositol 3-kinase

Pin1 - Peptidylprolyl cis/trans isomerase, NIMAinteracting 1

PINCH-1 - Particularly interesting cysteine-histidine rich protein-1

PKM2 - Pyruvate kinase M2

PML - Promyelocytic leukemia

Pnn - Pinin

POKEMON - POZ and Krüppel (POK) erythroid

myeloid ontogenic factor

PP2A - Protein phosphatase 2A

PRC2 - Polycomb-repressive complex 2

PTBP1 - Polypyrimidine tract binding protein

PTEN - Phosphatase and tensin homolog deleted on chromosome 10

PTPN2 - Protein tyrosine phosphatase, non-receptor type 2

Puma - p53-upregulated modulator of apoptosis

RACK1 - Receptor for activated C kinase 1

$\mathrm{Rb}$ - Retinoblastoma

RBP - RNA binding protein

ROS - Reactive oxygen species

RSK - Ribosomal S6 kinase

Runx - Runt-related transcription factor

SAHA - Suberoylanilide hydroxamic acid

SBHA - Suberoyl bis-hydroxamic acid

SCLC - Small-cell lung cancer

SGK - Serum- and Glucocorticoid-inducible kinase

SHIP1 - Src homology-2 domain-containing inositol 5-phosphatase 1

SIRT1 - Sirtuin 1

Smac - Second mitochondria-derived activator of caspase

Smad - Contraction of Sma and Mad (Mothers against decapentaplegic)

SNAI2 - Snail family zinc finger 2

SFSR6 (SRSF6) - Splicing factor arginine/serine

(RS)-rich 6

SHP2 - SH2 domain-containing phosphatase 2

SphK1 - Sphingosine kinase 1

SRSF1 (SFSR1) - Serine/arginine-rich splicing

factor-1

STAT-1 - Signal transducer and activator of transcription 1

SUZ12 - Suppressor of zeste 12 homolog

TCR - T cell receptor 
TGF $\beta$ - Transforming growth factor $\beta$

TKI - Tyrosine kinase inhibitor

TLR - Toll-like receptor

TNF $\alpha$ - Tumor necrosis factor $\alpha$

TRAIL - Tumor necrosis factor-related apoptosis-

inducing ligand

$\beta$-TrCP1 - Beta-transducin repeat containing E3

ubiquitin protein ligase 1

Treg - T regulatory cells

Trim33 - Tripartite motif containing 33

Trx-1 - Thioredoxin 1

Txnip - Thioredoxin-interacting protein

USP9X - Ubiquitin-Specific Protease 9X

3'-UTR - 3'-Untranslated region

VDAC - Voltage-dependent anion channel

YY-1 - Ying Yang 1

XIAP - X-linked inhibitor of apoptosis protein

\section{REFERENCES}

1. Willis SN and Adams JM. Life in the balance: how BH3only proteins induce apoptosis. Curr Opin Cell Biol. 2005; 17(6):617-625.

2. Bouillet P, Metcalf D, Huang DC, Tarlinton DM, Kay TW, Kontgen F, Adams JM and Strasser A. Proapoptotic Bcl2 relative Bim required for certain apoptotic responses, leukocyte homeostasis, and to preclude autoimmunity. Science. 1999; 286(5445):1735-1738.

3. O'Connor L, Strasser A, O'Reilly LA, Hausmann G, Adams JM, Cory S and Huang DC. Bim: a novel member of the Bcl-2 family that promotes apoptosis. EMBO J. 1998; 17(2):384-395.

4. Hsu SY, Lin P and Hsueh AJ. BOD (Bcl-2-related ovarian death gene) is an ovarian $\mathrm{BH} 3$ domain-containing proapoptotic $\mathrm{Bcl}-2$ protein capable of dimerization with diverse antiapoptotic Bcl-2 members. Mol Endocrinol. 1998; 12(9):1432-1440.

5. Jette CA, Flanagan AM, Ryan J, Pyati UJ, Carbonneau S, Stewart RA, Langenau DM, Look AT and Letai A. BIM and other BCL-2 family proteins exhibit cross-species conservation of function between zebrafish and mammals. Cell Death Differ. 2008; 15(6):1063-1072.

6. O'Reilly LA, Cullen L, Visvader J, Lindeman GJ, Print C, Bath ML, Huang DC and Strasser A. The proapoptotic BH3-only protein bim is expressed in hematopoietic, epithelial, neuronal, and germ cells. Am J Pathol. 2000; 157(2):449-461.

7. Harada H, Quearry B, Ruiz-Vela A and Korsmeyer SJ. Survival factor-induced extracellular signal-regulated kinase phosphorylates BIM, inhibiting its association with BAX and proapoptotic activity. Proc Natl Acad Sci U S A. 2004; 101(43):15313-15317.

8. Kuwana T, Bouchier-Hayes L, Chipuk JE, Bonzon C, Sullivan BA, Green DR and Newmeyer DD. BH3 domains of $\mathrm{BH} 3$-only proteins differentially regulate Bax-mediated mitochondrial membrane permeabilization both directly and indirectly. Mol Cell. 2005; 17(4):525-535.

9. Marani M, Tenev T, Hancock D, Downward J and Lemoine NR. Identification of novel isoforms of the BH3 domain protein Bim which directly activate Bax to trigger apoptosis. Mol Cell Biol. 2002; 22(11):3577-3589.

10. Gavathiotis E, Suzuki M, Davis ML, Pitter K, Bird GH, Katz SG, Tu HC, Kim H, Cheng EH, Tjandra $\mathrm{N}$ and Walensky LD. BAX activation is initiated at a novel interaction site. Nature. 2008; 455(7216):1076-1081.

11. Merino D, Giam M, Hughes PD, Siggs OM, Heger K, O'Reilly LA, Adams JM, Strasser A, Lee EF, Fairlie WD and Bouillet $\mathrm{P}$. The role of $\mathrm{BH} 3$-only protein Bim extends beyond inhibiting Bcl-2-like prosurvival proteins. J Cell Biol. 2009; 186(3):355-362.

12. Adams JM and Cory S. Bcl-2-regulated apoptosis: mechanism and therapeutic potential. Curr Opin Immunol. 2007; 19(5):488-496.

13. Sionov RV. MicroRNAs and Glucocorticoid-Induced Apoptosis in Lymphoid Malignancies. ISRN Hematol. 2013; 2013:348212.

14. Sionov RV, Spokoini R, Kfir-Erenfeld S, Cohen O and Yefenof E. Mechanisms regulating the susceptibility of hematopoietic malignancies to glucocorticoid-induced apoptosis. Adv Cancer Res. 2008; 101:127-248.

15. Rathmell JC, Lindsten T, Zong WX, Cinalli RM and Thompson CB. Deficiency in Bak and Bax perturbs thymic selection and lymphoid homeostasis. Nat Immunol. 2002; 3(10):932-939.

16. Bouillet P, Cory S, Zhang LC, Strasser A and Adams JM. Degenerative disorders caused by Bcl-2 deficiency prevented by loss of its BH3-only antagonist Bim. Dev Cell. 2001; 1(5):645-653.

17. Bouillet P, Zhang LC, Huang DC, Webb GC, Bottema CD, Shore P, Eyre HJ, Sutherland GR and Adams JM. Gene structure alternative splicing, and chromosomal localization of pro-apoptotic Bcl-2 relative Bim. Mamm Genome. 2001; 12(2):163-168.

18. Zhu Y, Swanson BJ, Wang M, Hildeman DA, Schaefer BC, Liu X, Suzuki H, Mihara K, Kappler J and Marrack P. Constitutive association of the proapoptotic protein Bim with Bcl-2-related proteins on mitochondria in T cells. Proc Natl Acad Sci U S A. 2004; 101(20):7681-7686.

19. Seward RJ, von Haller PD, Aebersold R and Huber BT. Phosphorylation of the pro-apoptotic protein Bim in lymphocytes is associated with protection from apoptosis. Mol Immunol. 2003; 39(16):983-993.

20. Kfir-Erenfeld S, Sionov RV, Spokoini R, Cohen O and Yefenof E. Protein kinase networks regulating glucocorticoid-induced apoptosis of hematopoietic cancer cells: fundamental aspects and practical considerations. Leuk Lymphoma. 2010; 51(11):1968-2005.

21. Abrams MT, Robertson NM, Yoon K and Wickstrom E. Inhibition of glucocorticoid-induced apoptosis by targeting 
the major splice variants of BIM mRNA with small interfering RNA and short hairpin RNA. J Biol Chem. 2004; 279(53):55809-55817.

22. Puthalakath H, Huang DC, O'Reilly LA, King SM and Strasser A. The proapoptotic activity of the Bcl-2 family member Bim is regulated by interaction with the dynein motor complex. Mol Cell. 1999; 3(3):287-296.

23. Letai A, Bassik MC, Walensky LD, Sorcinelli MD, Weiler $\mathrm{S}$ and Korsmeyer SJ. Distinct BH3 domains either sensitize or activate mitochondrial apoptosis, serving as prototype cancer therapeutics. Cancer Cell. 2002; 2(3):183-192.

24. Weber A, Paschen SA, Heger K, Wilfling F, Frankenberg T, Bauerschmitt H, Seiffert BM, Kirschnek S, Wagner H and Hacker G. BimS-induced apoptosis requires mitochondrial localization but not interaction with anti-apoptotic Bcl-2 proteins. J Cell Biol. 2007; 177(4):625-636.

25. Ley R, Ewings KE, Hadfield K and Cook SJ. Regulatory phosphorylation of Bim: sorting out the ERK from the JNK. Cell Death Differ. 2005; 12(8):1008-1014.

26. Shinjyo T, Kuribara R, Inukai T, Hosoi H, Kinoshita T, Miyajima A, Houghton PJ, Look AT, Ozawa K and Inaba T. Downregulation of Bim, a proapoptotic relative of Bcl2 , is a pivotal step in cytokine-initiated survival signaling in murine hematopoietic progenitors. Mol Cell Biol. 2001; 21(3):854-864.

27. Yamaguchi T, Okada T, Takeuchi K, Tonda T, Ohtaki M, Shinoda S, Masuzawa T, Ozawa K and Inaba T. Enhancement of thymidine kinase-mediated killing of malignant glioma by BimS, a BH3-only cell death activator. Gene Ther. 2003; 10(5):375-385.

28. U M, Miyashita T, Shikama Y, Tadokoro K and Yamada M. Molecular cloning and characterization of six novel isoforms of human Bim, a member of the proapoptotic Bcl2 family. FEBS Lett. 2001; 509(1):135-141.

29. Chen JZ, Ji CN, Gu SH, Li JX, Zhao EP, Huang Y, Huang L, Ying K, Xie Y and Mao YM. Over-expression of Bim alpha3, a novel isoform of human Bim, result in cell apoptosis. Int J Biochem Cell Biol. 2004; 36(8):1554-1561.

30. Liu JW, Chandra D, Tang SH, Chopra D and Tang DG. Identification and characterization of Bimgamma, a novel proapoptotic BH3-only splice variant of Bim. Cancer Res. 2002; 62(10):2976-2981.

31. Ng KP, Hillmer AM, Chuah CT, Juan WC, Ko TK, Teo AS, Ariyaratne PN, Takahashi N, Sawada K, Fei Y, Soh S, Lee WH, Huang JW, Allen JC, Jr., Woo XY, Nagarajan $\mathrm{N}$, et al. A common BIM deletion polymorphism mediates intrinsic resistance and inferior responses to tyrosine kinase inhibitors in cancer. Nat Med. 2012; 18(4):521-528.

32. Nakagawa T, Takeuchi S, Yamada T, Ebi H, Sano T, Nanjo S, Ishikawa D, Sato M, Hasegawa Y, Sekido Y and Yano S. EGFR-TKI resistance due to BIM polymorphism can be circumvented in combination with HDAC inhibition. Cancer Res. 2013; 73(8):2428-2434.

33. Chen X, Liu H, Xing H, Sun H and Zhu P. The BIM deletion polymorphism cannot account for intrinsic TKI resistance of Chinese individuals with chronic myeloid leukemia. Nat Med. 2014; 20(10):1090.

34. Gagne V, Rousseau J, Labuda M, Sharif-Askari B, Brukner I, Laverdiere C, Ceppi F, Sallan SE, Silverman LB, Neuberg D, Kutok JL, Sinnett D and Krajinovic M. Bim polymorphisms: influence on function and response to treatment in children with acute lymphoblastic leukemia. Clin Cancer Res. 2013; 19(18):5240-5249.

35. Puthalakath H, O'Reilly LA, Gunn P, Lee L, Kelly PN, Huntington ND, Hughes PD, Michalak EM, McKimmBreschkin J, Motoyama N, Gotoh T, Akira S, Bouillet P and Strasser A. ER stress triggers apoptosis by activating BH3-only protein Bim. Cell. 2007; 129(7):1337-1349.

36. Enders A, Bouillet P, Puthalakath H, Xu Y, Tarlinton DM and Strasser A. Loss of the pro-apoptotic BH3-only Bcl2 family member Bim inhibits BCR stimulation-induced apoptosis and deletion of autoreactive B cells. J Exp Med. 2003; 198(7):1119-1126.

37. Wang X, Szymczak-Workman AL, Gravano DM, Workman CJ, Green DR and Vignali DA. Preferential control of induced regulatory $\mathrm{T}$ cell homeostasis via a $\mathrm{Bim} / \mathrm{Bcl}-2$ axis. Cell Death Dis. 2012; 3:e270.

38. Chougnet CA, Tripathi P, Lages CS, Raynor J, Sholl A, Fink P, Plas DR and Hildeman DA. A major role for Bim in regulatory $\mathrm{T}$ cell homeostasis. J Immunol. 2011; 186(1):156-163.

39. Pierson W, Cauwe B, Policheni A, Schlenner SM, Franckaert D, Berges J, Humblet-Baron S, Schonefeldt S, Herold MJ, Hildeman D, Strasser A, Bouillet P, Lu LF, Matthys P, Freitas AA, Luther RJ, et al. Antiapoptotic Mcl-1 is critical for the survival and niche-filling capacity of Foxp3(+) regulatory T cells. Nat Immunol. 2013; 14(9):959-965.

40. Krishnamurthy B, Chee J, Jhala G, Trivedi P, Catterall T, Selck C, Gurzov EN, Brodnicki TC, Graham KL, Wali JA, Zhan Y, Gray D, Strasser A, Allison J, Thomas HE and Kay TW. Bim deficiency protects NOD mice from diabetes by diverting thymocytes to regulatory T cells. Diabetes. 2015; 64(9):3229-38.

41. Ludwinski MW, Sun J, Hilliard B, Gong S, Xue F, Carmody RJ, DeVirgiliis J and Chen YH. Critical roles of Bim in $\mathrm{T}$ cell activation and $\mathrm{T}$ cell-mediated autoimmune inflammation in mice. J Clin Invest. 2009; 119(6):17061713.

42. Ohgushi M, Kuroki S, Fukamachi H, O’Reilly LA, Kuida $\mathrm{K}$, Strasser A and Yonehara S. Transforming growth factor beta-dependent sequential activation of Smad, Bim, and caspase-9 mediates physiological apoptosis in gastric epithelial cells. Mol Cell Biol. 2005; 25(22):10017-10028.

43. Pellegrini M, Bouillet P, Robati M, Belz GT, Davey GM and Strasser A. Loss of Bim increases $\mathrm{T}$ cell production and function in interleukin 7 receptor-deficient mice. J Exp Med. 2004; 200(9):1189-1195. 
44. Oliver PM, Wang M, Zhu Y, White J, Kappler J and Marrack P. Loss of Bim allows precursor B cell survival but not precursor B cell differentiation in the absence of interleukin 7. J Exp Med. 2004; 200(9):1179-1187.

45. Akhtar RS, Klocke BJ, Strasser A and Roth KA. Loss of BH3-only protein Bim inhibits apoptosis of hemopoietic cells in the fetal liver and male germ cells but not neuronal cells in bcl-x-deficient mice. J Histochem Cytochem. 2008; 56(10):921-927.

46. Bouillet P, Purton JF, Godfrey DI, Zhang LC, Coultas L, Puthalakath H, Pellegrini M, Cory S, Adams JM and Strasser A. BH3-only Bcl-2 family member Bim is required for apoptosis of autoreactive thymocytes. Nature. 2002; 415(6874):922-926.

47. Hildeman DA, Zhu Y, Mitchell TC, Bouillet P, Strasser A, Kappler J and Marrack P. Activated T cell death in vivo mediated by proapoptotic bcl-2 family member bim. Immunity. 2002; 16(6):759-767.

48. Erlacher M, Labi V, Manzl C, Bock G, Tzankov A, Hacker G, Michalak E, Strasser A and Villunger A. Puma cooperates with Bim, the rate-limiting $\mathrm{BH} 3$-only protein in cell death during lymphocyte development, in apoptosis induction. J Exp Med. 2006; 203(13):2939-2951.

49. Erlacher M, Michalak EM, Kelly PN, Labi V, Niederegger H, Coultas L, Adams JM, Strasser A and Villunger A. BH3-only proteins Puma and Bim are rate-limiting for gamma-radiation- and glucocorticoid-induced apoptosis of lymphoid cells in vivo. Blood. 2005; 106(13):4131-4138.

50. Gray DH, Kupresanin F, Berzins SP, Herold MJ, O'Reilly LA, Bouillet $\mathrm{P}$ and Strasser A. The BH3-only proteins Bim and Puma cooperate to impose deletional tolerance of organ-specific antigens. Immunity. 2012; 37(3):451-462.

51. Huntington ND, Labi V, Cumano A, Vieira P, Strasser A, Villunger A, Di Santo JP and Alves NL. Loss of the proapoptotic BH3-only Bcl-2 family member Bim sustains B lymphopoiesis in the absence of IL-7. Int Immunol. 2009; 21(6):715-725.

52. Coultas L, Bouillet P, Loveland KL, Meachem S, Perlman H, Adams JM and Strasser A. Concomitant loss of proapoptotic BH3-only Bcl-2 antagonists Bik and Bim arrests spermatogenesis. EMBO J. 2005; 24(22):3963-3973.

53. Strasser A. The role of BH3-only proteins in the immune system. Nat Rev Immunol. 2005; 5(3):189-200.

54. Zong WX, Lindsten T, Ross AJ, MacGregor GR and Thompson CB. BH3-only proteins that bind pro-survival Bcl-2 family members fail to induce apoptosis in the absence of Bax and Bak. Genes Dev. 2001; 15(12):14811486.

55. Wei MC, Zong WX, Cheng EH, Lindsten T, Panoutsakopoulou V, Ross AJ, Roth KA, MacGregor GR, Thompson CB and Korsmeyer SJ. Proapoptotic BAX and BAK: a requisite gateway to mitochondrial dysfunction and death. Science. 2001; 292(5517):727-730.

56. Chipuk JE, Moldoveanu T, Llambi F, Parsons MJ and
Green DR. The BCL-2 family reunion. Mol Cell. 2010; 37(3):299-310.

57. Hagenbuchner J and Ausserlechner MJ. Mitochondria and FOXO3: breath or die. Front Physiol. 2013; 4:147.

58. Willis SN, Fletcher JI, Kaufmann T, van Delft MF, Chen L, Czabotar PE, Ierino H, Lee EF, Fairlie WD, Bouillet P, Strasser A, Kluck RM, Adams JM and Huang DC. Apoptosis initiated when BH3 ligands engage multiple Bcl2 homologs, not Bax or Bak. Science. 2007; 315(5813):856859.

59. Zhang L, Xing D and Chen M. Bim(L) displacing Bcl$\mathrm{x}(\mathrm{L})$ promotes Bax translocation during TNFalpha-induced apoptosis. Apoptosis. 2008; 13(7):950-958.

60. Wang X, Xing D, Liu L and Chen WR. BimL directly neutralizes Bcl-xL to promote Bax activation during UVinduced apoptosis. FEBS Lett. 2009; 583(12):1873-1879.

61. Hsu YT, Wolter KG and Youle RJ. Cytosol-to-membrane redistribution of Bax and $\mathrm{Bcl}-\mathrm{X}(\mathrm{L})$ during apoptosis. Proc Natl Acad Sci U S A. 1997; 94(8):3668-3672.

62. Wolter KG, Hsu YT, Smith CL, Nechushtan A, Xi $\mathrm{XG}$ and Youle RJ. Movement of Bax from the cytosol to mitochondria during apoptosis. J Cell Biol. 1997; 139(5):1281-1292.

63. Spokoini R, Kfir-Erenfeld S, Yefenof E and Sionov RV. Glycogen synthase kinase-3 plays a central role in mediating glucocorticoid-induced apoptosis. Mol Endocrinol. 2010; 24(6):1136-1150.

64. Kfir S, Sionov RV, Zafrir E, Zilberman Y and Yefenof E. Staurosporine sensitizes $\mathrm{T}$ lymphoma cells to glucocorticoid-induced apoptosis: role of Nur77 and Bcl-2. Cell Cycle. 2007; 6(24):3086-3096.

65. Sionov RV, Cohen O, Kfir S, Zilberman Y and Yefenof E. Role of mitochondrial glucocorticoid receptor in glucocorticoid-induced apoptosis. J Exp Med. 2006; 203(1):189-201.

66. Yamaguchi $\mathrm{H}$ and Wang HG. Bcl-XL protects BimELinduced Bax conformational change and cytochrome $\mathrm{C}$ release independent of interacting with Bax or BimEL. J Biol Chem. 2002; 277(44):41604-41612.

67. Green DR and Chipuk JE. Apoptosis: Stabbed in the BAX. Nature. 2008; 455(7216):1047-1049.

68. Ren D, Tu HC, Kim H, Wang GX, Bean GR, Takeuchi O, Jeffers JR, Zambetti GP, Hsieh JJ and Cheng EH. BID, BIM, and PUMA are essential for activation of the BAXand BAK-dependent cell death program. Science. 2010; 330(6009):1390-1393.

69. Kim H, Tu HC, Ren D, Takeuchi O, Jeffers JR, Zambetti GP, Hsieh JJ and Cheng EH. Stepwise activation of BAX and BAK by tBID, BIM, and PUMA initiates mitochondrial apoptosis. Mol Cell. 2009; 36(3):487-499.

70. Suzuki M, Youle RJ and Tjandra N. Structure of Bax: coregulation of dimer formation and intracellular localization. Cell. 2000; 103(4):645-654.

71. Cheng EH, Sheiko TV, Fisher JK, Craigen WJ and 
Korsmeyer SJ. VDAC2 inhibits BAK activation and mitochondrial apoptosis. Science. 2003; 301(5632):513517.

72. Ren D, Kim H, Tu HC, Westergard TD, Fisher JK, Rubens JA, Korsmeyer SJ, Hsieh JJ and Cheng EH. The VDAC2BAK rheostat controls thymocyte survival. Sci Signal. 2009; 2(85):ra48.

73. Gavathiotis E, Reyna DE, Davis ML, Bird GH and Walensky LD. BH3-triggered structural reorganization drives the activation of proapoptotic BAX. Mol Cell. 2010; 40(3):481-492.

74. Dewson G, Kratina T, Sim HW, Puthalakath H, Adams JM, Colman PM and Kluck RM. To trigger apoptosis, Bak exposes its $\mathrm{BH} 3$ domain and homodimerizes via BH3:groove interactions. Mol Cell. 2008; 30(3):369-380.

75. Dewson G, Kratina T, Czabotar P, Day CL, Adams JM and Kluck RM. Bak activation for apoptosis involves oligomerization of dimers via their alpha6 helices. Mol Cell. 2009; 36(4):696-703.

76. Sarosiek KA, Chi X, Bachman JA, Sims JJ, Montero J, Patel L, Flanagan A, Andrews DW, Sorger P and Letai A. BID preferentially activates BAK while BIM preferentially activates BAX, affecting chemotherapy response. Mol Cell. 2013; 51(6):751-765.

77. Walensky LD, Pitter K, Morash J, Oh KJ, Barbuto S, Fisher J, Smith E, Verdine GL and Korsmeyer SJ. A stapled BID BH3 helix directly binds and activates BAX. Mol Cell. 2006; 24(2):199-210.

78. Weber K, Harper N, Schwabe J and Cohen GM. BIMmediated membrane insertion of the BAK pore domain is an essential requirement for apoptosis. Cell Rep. 2013; 5(2):409-420.

79. Dai H, Pang YP, Ramirez-Alvarado M and Kaufmann SH. Evaluation of the BH3-only Protein Puma as a Direct Bak Activator. J Biol Chem. 2014; 289(1):89-99.

80. Vela L, Gonzalo O, Naval J and Marzo I. Direct interaction of Bax and Bak proteins with Bcl-2 homology domain 3 (BH3)-only proteins in living cells revealed by fluorescence complementation. J Biol Chem. 2013; 288(7):4935-4946.

81. Zhang Y, Xing D and Liu L. PUMA promotes Bax translocation by both directly interacting with Bax and by competitive binding to Bcl-X L during UV-induced apoptosis. Mol Biol Cell. 2009; 20(13):3077-3087.

82. Savry A, Carre M, Berges R, Rovini A, Pobel I, Chacon C, Braguer D and Bourgarel-Rey V. Bcl-2-enhanced efficacy of microtubule-targeting chemotherapy through Bim overexpression: implications for cancer treatment. Neoplasia. 2013; 15(1):49-60.

83. Mouhamad S, Besnault L, Auffredou MT, Leprince C, Bourgeade MF, Leca G and Vazquez A. B cell receptormediated apoptosis of human lymphocytes is associated with a new regulatory pathway of Bim isoform expression. J Immunol. 2004; 172(4):2084-2091.

84. Kuroda $\mathrm{J}$ and Taniwaki $\mathrm{M}$. Involvement of BH3-only proteins in hematologic malignancies. Crit Rev Oncol Hematol. 2009; 71(2):89-101.

85. Gomez-Bougie P, Bataille R and Amiot M. Endogenous association of Bim BH3-only protein with Mcl-1, Bcl$\mathrm{xL}$ and Bcl-2 on mitochondria in human B cells. Eur J Immunol. 2005; 35(3):971-976.

86. Gomez-Bougie P, Bataille R and Amiot M. The imbalance between Bim and Mcl-1 expression controls the survival of human myeloma cells. Eur J Immunol. 2004; 34(11):31563164.

87. Luo S, Garcia-Arencibia M, Zhao R, Puri C, Toh PP, Sadiq $\mathrm{O}$ and Rubinsztein DC. Bim inhibits autophagy by recruiting Beclin 1 to microtubules. Mol Cell. 2012; 47(3):359-370.

88. Luo S and Rubinsztein DC. BCL2L11/BIM: a novel molecular link between autophagy and apoptosis. Autophagy. 2013; 9(1):104-105.

89. Ruppert SM, Li W, Zhang G, Carlson AL, Limaye A, Durum SK and Khaled AR. The major isoforms of Bim contribute to distinct biological activities that govern the processes of autophagy and apoptosis in interleukin-7 dependent lymphocytes. Biochim Biophys Acta. 2012; 1823(10):1877-1893.

90. Lei K and Davis RJ. JNK phosphorylation of Bim-related members of the Bcl2 family induces Bax-dependent apoptosis. Proc Natl Acad Sci U S A. 2003; 100(5):24322437.

91. Puthalakath H, Villunger A, O'Reilly LA, Beaumont JG, Coultas L, Cheney RE, Huang DC and Strasser A. Bmf: a proapoptotic $\mathrm{BH} 3$-only protein regulated by interaction with the myosin $\mathrm{V}$ actin motor complex, activated by anoikis. Science. 2001; 293(5536):1829-1832.

92. Tong T, Ji J, Jin S, Li X, Fan W, Song Y, Wang M, Liu $\mathrm{Z}, \mathrm{Wu} \mathrm{M}$ and Zhan Q. Gadd45a expression induces Bim dissociation from the cytoskeleton and translocation to mitochondria. Mol Cell Biol. 2005; 25(11):4488-4500.

93. Talapatra S, Wagner JD and Thompson CB. Elongation factor-1 alpha is a selective regulator of growth factor withdrawal and ER stress-induced apoptosis. Cell Death Differ. 2002; 9(8):856-861.

94. Blanch A, Robinson F, Watson IR, Cheng LS and Irwin MS. Eukaryotic translation elongation factor 1-alpha 1 inhibits p53 and p73 dependent apoptosis and chemotherapy sensitivity. PLoS One. 2013; 8(6):e66436.

95. Han J, Goldstein LA, Gastman BR and Rabinowich H. Interrelated roles for $\mathrm{Mcl}-1$ and BIM in regulation of TRAIL-mediated mitochondrial apoptosis. J Biol Chem. 2006; 281(15):10153-10163.

96. Herrant M, Jacquel A, Marchetti S, Belhacene N, Colosetti P, Luciano F and Auberger P. Cleavage of Mcl-1 by caspases impaired its ability to counteract Bim-induced apoptosis. Oncogene. 2004; 23(47):7863-7873.

97. Han J, Goldstein LA, Gastman BR, Froelich CJ, Yin XM and Rabinowich H. Degradation of Mcl-1 by granzyme B: 
implications for Bim-mediated mitochondrial apoptotic events. J Biol Chem. 2004; 279(21):22020-22029.

98. Iglesias-Serret D, Pique M, Gil J, Pons G and Lopez JM. Transcriptional and translational control of Mcl-1 during apoptosis. Arch Biochem Biophys. 2003; 417(2):141-152.

99. Craig RW. MCL1 provides a window on the role of the BCL2 family in cell proliferation, differentiation and tumorigenesis. Leukemia. 2002; 16(4):444-454.

100. Lee EF, Czabotar PE, van Delft MF, Michalak EM, Boyle MJ, Willis SN, Puthalakath H, Bouillet P, Colman PM, Huang DC and Fairlie WD. A novel BH3 ligand that selectively targets Mcl-1 reveals that apoptosis can proceed without Mcl-1 degradation. J Cell Biol. 2008; 180(2):341355.

101. Zhong Q, Gao W, Du F and Wang X. Mule/ARFBP1, a BH3-only E3 ubiquitin ligase, catalyzes the polyubiquitination of Mcl-1 and regulates apoptosis. Cell. 2005; 121(7):1085-1095.

102. Ding Q, He X, Hsu JM, Xia W, Chen CT, Li LY, Lee DF, Liu JC, Zhong Q, Wang X and Hung MC. Degradation of Mcl-1 by beta-TrCP mediates glycogen synthase kinase 3 -induced tumor suppression and chemosensitization. Mol Cell Biol. 2007; 27(11):4006-4017.

103. Mojsa B, Lassot I and Desagher S. Mcl-1 ubiquitination: unique regulation of an essential survival protein. Cells. 2014; 3(2):418-437.

104. Magiera MM, Mora S, Mojsa B, Robbins I, Lassot I and Desagher S. Trim17-mediated ubiquitination and degradation of Mcl-1 initiate apoptosis in neurons. Cell Death Differ. 2013; 20(2):281-292.

105. Mills JR, Hippo Y, Robert F, Chen SM, Malina A, Lin CJ, Trojahn U, Wendel HG, Charest A, Bronson RT, Kogan SC, Nadon R, Housman DE, Lowe SW and Pelletier J. mTORC1 promotes survival through translational control of Mcl-1. Proc Natl Acad Sci U S A. 2008; 105(31):1085310858

106. Inuzuka H, Shaik S, Onoyama I, Gao D, Tseng A, Maser RS, Zhai B, Wan L, Gutierrez A, Lau AW, Xiao Y, Christie AL, Aster J, Settleman J, Gygi SP, Kung AL, et al. SCF(FBW7) regulates cellular apoptosis by targeting MCL1 for ubiquitylation and destruction. Nature. 2011; 471(7336):104-109.

107. Wertz IE, Kusam S, Lam C, Okamoto T, Sandoval W, Anderson DJ, Helgason E, Ernst JA, Eby M, Liu J, Belmont LD, Kaminker JS, O'Rourke KM, Pujara K, Kohli PB, Johnson AR, et al. Sensitivity to antitubulin chemotherapeutics is regulated by MCL1 and FBW7. Nature. 2011; 471(7336):110-114.

108. Koo J, Yue P, Deng X, Khuri FR and Sun SY. mTOR Complex 2 Stabilizes Mcl-1 Protein by Suppressing Its Glycogen Synthase Kinase 3-Dependent and SCF-FBXW7Mediated Degradation. Mol Cell Biol. 2015; 35(13):23442355.

109. Xiao K, Chen P and Chang DC. The VTLISFG motif in the BH1 domain plays a significant role in regulating the degradation of Mcl-1. FEBS Open Bio. 2014; 4:147-152.

110. Schwickart M, Huang X, Lill JR, Liu J, Ferrando R, French DM, Maecker H, O'Rourke K, Bazan F, EasthamAnderson J, Yue P, Dornan D, Huang DC and Dixit VM. Deubiquitinase USP9X stabilizes MCL1 and promotes tumour cell survival. Nature. 2010; 463(7277):103-107.

111. Zhou M, Wang T, Lai H, Zhao X, Yu Q, Zhou J and Yang Y. Targeting of the deubiquitinase USP9X attenuates B-cell acute lymphoblastic leukemia cell survival and overcomes glucocorticoid resistance. Biochem Biophys Res Commun. 2015; 125(23):3588-3597.

112. Warr MR, Acoca S, Liu Z, Germain M, Watson M, Blanchette M, Wing SS and Shore GC. BH3-ligand regulates access of MCL-1 to its E3 ligase. FEBS Lett. 2005; 579(25):5603-5608.

113. Gomez-Bougie P, Menoret E, Juin P, Dousset C, PellatDeceunynck $\mathrm{C}$ and Amiot M. Noxa controls Muledependent Mcl-1 ubiquitination through the regulation of the Mcl-1/USP9X interaction. Biochem Biophys Res Commun. 2011; 413(3):460-464.

114. Elgendy M, Ciro M, Abdel-Aziz AK, Belmonte G, Dal Zuffo R, Mercurio C, Miracco C, Lanfrancone L, Foiani $\mathrm{M}$ and Minucci S. Beclin 1 restrains tumorigenesis through Mcl-1 destabilization in an autophagy-independent reciprocal manner. Nat Commun. 2014; 5:5637.

115. Peddaboina C, Jupiter D, Fletcher S, Yap JL, Rai A, Tobin RP, Jiang W, Rascoe P, Rogers MK, Smythe WR and Cao $\mathrm{X}$. The downregulation of Mcl-1 via USP9X inhibition sensitizes solid tumors to Bcl-xl inhibition. BMC Cancer. 2012; $12: 541$.

116. Biswas SC, Shi Y, Sproul A and Greene LA. Proapoptotic Bim induction in response to nerve growth factor deprivation requires simultaneous activation of three different death signaling pathways. J Biol Chem. 2007; 282(40):29368-29374.

117. Gilley J, Coffer PJ and Ham J. FOXO transcription factors directly activate bim gene expression and promote apoptosis in sympathetic neurons. J Cell Biol. 2003; 162(4):613-622.

118. Sunters A, Fernandez de Mattos S, Stahl M, Brosens JJ, Zoumpoulidou G, Saunders CA, Coffer PJ, Medema $\mathrm{RH}$, Coombes RC and Lam EW. FoxO3a transcriptional regulation of Bim controls apoptosis in paclitaxel-treated breast cancer cell lines. J Biol Chem. 2003; 278(50):4979549805.

119. Gilley J and Ham J. Evidence for increased complexity in the regulation of Bim expression in sympathetic neurons: involvement of novel transcriptional and translational mechanisms. DNA Cell Biol. 2005; 24(9):563-573.

120. Xie B, Wang C, Zheng Z, Song B, Ma C, Thiel G and Li M. Egr-1 transactivates Bim gene expression to promote neuronal apoptosis. J Neurosci. 2011; 31(13):5032-5044.

121. Essafi A, Fernandez de Mattos S, Hassen YA, Soeiro I, Mufti GJ, Thomas NS, Medema RH and Lam EW. Direct 
transcriptional regulation of $\mathrm{Bim}$ by FoxO3a mediates STI571-induced apoptosis in Bcr-Abl-expressing cells. Oncogene. 2005; 24(14):2317-2329.

122. Dijkers PF, Medema RH, Lammers JW, Koenderman L and Coffer PJ. Expression of the pro-apoptotic Bcl-2 family member Bim is regulated by the forkhead transcription factor FKHR-L1. Curr Biol. 2000; 10(19):1201-1204.

123. Obexer P, Geiger K, Ambros PF, Meister B and Ausserlechner MJ. FKHRL1-mediated expression of Noxa and Bim induces apoptosis via the mitochondria in neuroblastoma cells. Cell Death Differ. 2007; 14(3):534547.

124. Li Z, Zhang J, Liu Z, Woo CW and Thiele CJ. Downregulation of Bim by brain-derived neurotrophic factor activation of TrkB protects neuroblastoma cells from paclitaxel but not etoposide or cisplatin-induced cell death. Cell Death Differ. 2007; 14(2):318-326.

125. Whitfield J, Neame SJ, Paquet L, Bernard O and Ham J. Dominant-negative c-Jun promotes neuronal survival by reducing BIM expression and inhibiting mitochondrial cytochrome c release. Neuron. 2001; 29(3):629-643.

126. Jiang Y, Zou L, Lu WQ, Zhang Y and Shen AG. Foxo3a expression is a prognostic marker in breast cancer. PLoS One. 2013; 8(8):e70746.

127. Habashy HO, Rakha EA, Aleskandarany M, Ahmed MA, Green AR, Ellis IO and Powe DG. FOXO3a nuclear localisation is associated with good prognosis in luminallike breast cancer. Breast Cancer Res Treat. 2011; 129(1):11-21.

128. Shou Z, Lin L, Liang J, Li JL and Chen HY. Expression and prognosis of FOXO3a and HIF-1alpha in nasopharyngeal carcinoma. J Cancer Res Clin Oncol. 2012; 138(4):585-593.

129. Carbajo-Pescador S, Mauriz JL, Garcia-Palomo A and Gonzalez-Gallego J. FoxO Proteins: Regulation and Molecular Targets in Liver Cancer. Curr Med Chem. 2013.

130. Yang XB, Zhao JJ, Huang CY, Wang QJ, Pan K, Wang DD, Pan QZ, Jiang SS, Lv L, Gao X, Chen HW, Yao JY, Zhi M and Xia JC. Decreased expression of the FOXO3a gene is associated with poor prognosis in primary gastric adenocarcinoma patients. PLoS One. 2013; 8(10):e78158.

131. Shukla S, Shukla M, Maclennan GT, Fu P and Gupta S. Deregulation of FOXO3A during prostate cancer progression. Int J Oncol. 2009; 34(6):1613-1620.

132. Yang JY, Zong CS, Xia W, Yamaguchi H, Ding Q, Xie X, Lang JY, Lai CC, Chang CJ, Huang WC, Huang H, Kuo HP, Lee DF, Li LY, Lien HC, Cheng X, et al. ERK promotes tumorigenesis by inhibiting FOXO3a via MDM2mediated degradation. Nat Cell Biol. 2008; 10(2):138-148.

133. Wang YQ, Cao Q, Wang F, Huang LY, Sang TT, Liu F and Chen SY. SIRT1 protects against oxidative stressinduced endothelial progenitor cells apoptosis by inhibiting FOXO3a via FOXO3a ubiquitination and degradation. J Cell Physiol. 2015; 230(9):2098-2107.

134. Hu MC, Lee DF, Xia W, Golfman LS, Ou-Yang F, Yang
JY, Zou Y, Bao S, Hanada N, Saso H, Kobayashi R and Hung MC. IkappaB kinase promotes tumorigenesis through inhibition of forkhead FOXO3a. Cell. 2004; 117(2):225237.

135. Plas DR and Thompson CB. Akt activation promotes degradation of tuberin and FOXO3a via the proteasome. $\mathrm{J}$ Biol Chem. 2003; 278(14):12361-12366.

136. Brunet A, Bonni A, Zigmond MJ, Lin MZ, Juo P, Hu LS, Anderson MJ, Arden KC, Blenis J and Greenberg ME. Akt promotes cell survival by phosphorylating and inhibiting a Forkhead transcription factor. Cell. 1999; 96(6):857-868.

137. Sunayama J, Sato A, Matsuda K, Tachibana K, Watanabe E, Seino S, Suzuki K, Narita Y, Shibui S, Sakurada K, Kayama T, Tomiyama A and Kitanaka C. FoxO3a functions as a key integrator of cellular signals that control glioblastoma stemlike cell differentiation and tumorigenicity. Stem Cells. 2011; 29(9):1327-1337.

138. Ho KK, McGuire VA, Koo CY, Muir KW, de Olano N, Maifoshie E, Kelly DJ, McGovern UB, Monteiro LJ, Gomes AR, Nebreda AR, Campbell DG, Arthur JS and Lam EW. Phosphorylation of FOXO3a on Ser-7 by p38 promotes its nuclear localization in response to doxorubicin. J Biol Chem. 2012; 287(2):1545-1555.

139. Cai B and Xia Z. p38 MAP kinase mediates arseniteinduced apoptosis through FOXO3a activation and induction of Bim transcription. Apoptosis. 2008; 13(6):803810.

140. Chatterjee A, Chatterjee U and Ghosh MK. Activation of protein kinase CK2 attenuates FOXO3a functioning in a PML-dependent manner: implications in human prostate cancer. Cell Death Dis. 2013; 4:e543.

141. Scaglioni PP, Yung TM, Cai LF, Erdjument-Bromage H, Kaufman AJ, Singh B, Teruya-Feldstein J, Tempst P and Pandolfi PP. A CK2-dependent mechanism for degradation of the PML tumor suppressor. Cell. 2006; 126(2):269-283.

142. Trotman LC, Alimonti A, Scaglioni PP, Koutcher JA, Cordon-Cardo C and Pandolfi PP. Identification of a tumour suppressor network opposing nuclear Akt function. Nature. 2006; 441(7092):523-527.

143. Karadedou CT, Gomes AR, Chen J, Petkovic M, Ho KK, Zwolinska AK, Feltes A, Wong SY, Chan KY, Cheung YN, Tsang JW, Brosens JJ, Khoo US and Lam EW. FOXO3a represses VEGF expression through FOXM1-dependent and -independent mechanisms in breast cancer. Oncogene. 2012; 31(14):1845-1858.

144. Stahl M, Dijkers PF, Kops GJ, Lens SM, Coffer PJ, Burgering BM and Medema RH. The forkhead transcription factor FoxO regulates transcription of $\mathrm{p} 27 \mathrm{Kip} 1$ and Bim in response to IL-2. J Immunol. 2002; 168(10):5024-5031.

145. Herold MJ, Rohrbeck L, Lang MJ, Grumont R, Gerondakis S, Tai L, Bouillet P, Kaufmann T and Strasser A. Foxomediated Bim transcription is dispensable for the apoptosis of hematopoietic cells that is mediated by this BH3-only protein. EMBO Rep. 2013; 14(11):992-998. 
146. Koenig MN, Naik E, Rohrbeck L, Herold MJ, Trounson E, Bouillet P, Thomas T, Voss AK, Strasser A and Coultas L. Pro-apoptotic BIM is an essential initiator of physiological endothelial cell death independent of regulation by FOXO3. Cell Death Differ. 2014; 21(11):1687-1695.

147. Sullivan JA, Kim EH, Plisch EH, Peng SL and Suresh M. FOXO3 regulates CD8 $\mathrm{T}$ cell memory by $\mathrm{T}$ cell-intrinsic mechanisms. PLoS Pathog. 2012; 8(2):e1002533.

148. Miyamoto K, Miyamoto $\mathrm{T}$, Kato R, Yoshimura A, Motoyama $\mathrm{N}$ and Suda T. FoxO3a regulates hematopoietic homeostasis through a negative feedback pathway in conditions of stress or aging. Blood. 2008; 112(12):44854493.

149. Tothova Z, Kollipara R, Huntly BJ, Lee BH, Castrillon DH, Cullen DE, McDowell EP, Lazo-Kallanian S, Williams IR, Sears C, Armstrong SA, Passegue E, DePinho RA and Gilliland DG. FoxOs are critical mediators of hematopoietic stem cell resistance to physiologic oxidative stress. Cell. 2007; 128(2):325-339.

150. Hughes R, Kristiansen M, Lassot I, Desagher S, Mantovani $\mathrm{R}$ and Ham J. NF-Y is essential for expression of the proapoptotic bim gene in sympathetic neurons. Cell Death Differ. 2011; 18(6):937-947.

151. Wang Y, Wan C, Yu S, Yang L, Li B, Lu T, Bi Y, Jiang J and Cui G. Upregulated expression of NF-YC contributes to neuronal apoptosis via proapoptotic protein bim in rats' brain hippocampus following middle cerebral artery occlusion (MCAO). J Mol Neurosci. 2014; 52(4):552-565.

152. Wildey GM, Patil S and Howe PH. Smad3 potentiates transforming growth factor beta (TGFbeta)-induced apoptosis and expression of the $\mathrm{BH} 3$-only protein Bim in WEHI 231 B lymphocytes. J Biol Chem. 2003; 278(20):18069-18077.

153. Ha Thi HT, Lim HS, Kim J, Kim YM, Kim HY and Hong S. Transcriptional and post-translational regulation of Bim is essential for TGF-beta and TNF-alpha-induced apoptosis of gastric cancer cell. Biochim Biophys Acta. 2013; 1830(6):3584-3592.

154. Yu J, Zhang L, Chen A, Xiang G, Wang Y, Wu J, Mitchelson K, Cheng $\mathrm{J}$ and Zhou Y. Identification of the gene transcription and apoptosis mediated by TGFbeta-Smad2/3-Smad4 signaling. J Cell Physiol. 2008; 215(2):422-433.

155. Zhao X, Liu Y, Du L, He L, Ni B, Hu J, Zhu D and Chen Q. Threonine 32 (Thr32) of FoxO3 is critical for TGFbeta-induced apoptosis via Bim in hepatocarcinoma cells. Protein Cell. 2015; 6(2):127-138.

156. Putcha GV, Le S, Frank S, Besirli CG, Clark K, Chu B, Alix S, Youle RJ, LaMarche A, Maroney AC and Johnson EM, Jr. JNK-mediated BIM phosphorylation potentiates BAXdependent apoptosis. Neuron. 2003; 38(6):899-914.

157. Shi L, Gong S, Yuan Z, Ma C, Liu Y, Wang C, Li W, Pi R, Huang S, Chen R, Han Y, Mao Z and Li M. Activity deprivation-dependent induction of the proapoptotic $\mathrm{BH} 3$ - only protein Bim is independent of $\mathrm{JNK} / \mathrm{c}-\mathrm{Jun}$ activation during apoptosis in cerebellar granule neurons. Neurosci Lett. 2005; 375(1):7-12.

158. Lu J, Quearry B and Harada H. p38-MAP kinase activation followed by BIM induction is essential for glucocorticoidinduced apoptosis in lymphoblastic leukemia cells. FEBS Lett. 2006; 580(14):3539-3544.

159. Heidari N, Miller AV, Hicks MA, Marking CB and Harada H. Glucocorticoid-mediated BIM induction and apoptosis are regulated by Runx 2 and c-Jun in leukemia cells. Cell Death Dis. 2012; 3:e349.

160. Chen DW, Saha V, Liu JZ, Schwartz JM and KrsticDemonacos M. Erg and AP-1 as determinants of glucocorticoid response in acute lymphoblastic leukemia. Oncogene. 2013; 32(25):3039-3048.

161. Nuutinen U, Ropponen A, Suoranta S, Eeva J, Eray M, Pellinen R, Wahlfors J and Pelkonen J. Dexamethasoneinduced apoptosis and up-regulation of Bim is dependent on glycogen synthase kinase-3. Leuk Res. 2009; 33(12):17141717.

162. Chimge NO, Baniwal SK, Little GH, Chen YB, Kahn M, Tripathy D, Borok Z and Frenkel B. Regulation of breast cancer metastasis by Runx2 and estrogen signaling: the role of SNAI2. Breast Cancer Res. 2011; 13(6):R127.

163. Merino D, Best SA, Asselin-Labat ML, Vaillant F, Pal B, Dickins RA, Anderson RL, Strasser A, Bouillet P, Lindeman GJ and Visvader JE. Pro-apoptotic Bim suppresses breast tumor cell metastasis and is a target gene of SNAI2. Oncogene. 2015; 34(30):3926-3934.

164. Yano T, Ito K, Fukamachi H, Chi XZ, Wee HJ, Inoue K, Ida H, Bouillet P, Strasser A, Bae SC and Ito Y. The RUNX3 tumor suppressor upregulates Bim in gastric epithelial cells undergoing transforming growth factor beta-induced apoptosis. Mol Cell Biol. 2006; 26(12):4474-4488.

165. Wildey GM and Howe PH. Runx1 is a co-activator with FOXO3 to mediate transforming growth factor beta (TGFbeta)-induced Bim transcription in hepatic cells. J Biol Chem. 2009; 284(30):20227-20239.

166. Ramesh S, Qi XJ, Wildey GM, Robinson J, Molkentin $\mathrm{J}$, Letterio $\mathrm{J}$ and Howe PH. TGF beta-mediated BIM expression and apoptosis are regulated through SMAD3dependent expression of the MAPK phosphatase MKP2. EMBO Rep. 2008; 9(10):990-997.

167. Abe N, Kohu K, Ohmori H, Hayashi K, Watanabe T, Hozumi K, Sato T, Habu S and Satake M. Reduction of Runx1 transcription factor activity up-regulates Fas and Bim expression and enhances the apoptotic sensitivity of double positive thymocytes. J Immunol. 2005; 175(7):44754482 .

168. Hershko $\mathrm{T}$ and Ginsberg D. Up-regulation of Bcl-2 homology 3 (BH3)-only proteins by E2F1 mediates apoptosis. J Biol Chem. 2004; 279(10):8627-8634.

169. Zhao Y, Tan J, Zhuang L, Jiang X, Liu ET and Yu Q. Inhibitors of histone deacetylases target the $\mathrm{Rb}-\mathrm{E} 2 \mathrm{~F} 1$ 
pathway for apoptosis induction through activation of proapoptotic protein Bim. Proc Natl Acad Sci U S A. 2005; 102(44):16090-16095.

170. Tan J, Zhuang L, Jiang X, Yang KK, Karuturi KM and Yu Q. Apoptosis signal-regulating kinase 1 is a direct target of E2F1 and contributes to histone deacetylase inhibitorinduced apoptosis through positive feedback regulation of E2F1 apoptotic activity. J Biol Chem. 2006; 281(15):1050810515.

171. Wu J, Kharebava G, Piao C, Stoica BA, Dinizo M, Sabirzhanov B, Hanscom M, Guanciale K and Faden AI. Inhibition of E2F1/CDK1 pathway attenuates neuronal apoptosis in vitro and confers neuroprotection after spinal cord injury in vivo. PLoS One. 2012; 7(7):e42129.

172. Kim YC and Day RM. Angiotensin II regulates activation of Bim via Rb/E2F1 during apoptosis: involvement of interaction between AMPKbeta1/2 and Cdk4. Am J Physiol Lung Cell Mol Physiol. 2012; 303(3):L228-238.

173. Wu ZL, Zheng SS, Li ZM, Qiao YY, Aau MY and Yu Q. Polycomb protein EZH2 regulates E2F1-dependent apoptosis through epigenetically modulating Bim expression. Cell Death Differ. 2010; 17(5):801-810.

174. Fujii S, Ito K, Ito $\mathrm{Y}$ and Ochiai A. Enhancer of zeste homologue 2 (EZH2) down-regulates RUNX3 by increasing histone H3 methylation. J Biol Chem. 2008; 283(25):17324-17332.

175. Petrocca F, Visone R, Onelli MR, Shah MH, Nicoloso MS, de Martino I, Iliopoulos D, Pilozzi E, Liu CG, Negrini M, Cavazzini L, Volinia S, Alder H, Ruco LP, Baldassarre $\mathrm{G}$, Croce CM, et al. E2F1-regulated microRNAs impair TGFbeta-dependent cell-cycle arrest and apoptosis in gastric cancer. Cancer Cell. 2008; 13(3):272-286.

176. Gogada R, Yadav N, Liu J, Tang S, Zhang D, Schneider A, Seshadri A, Sun L, Aldaz CM, Tang DG and Chandra D. Bim, a proapoptotic protein, up-regulated via transcription factor E2F1-dependent mechanism, functions as a prosurvival molecule in cancer. J Biol Chem. 2013; 288(1):368-381.

177. Wu M, Kalyanasundaram A and Zhu J. Structural and biomechanical basis of mitochondrial movement in eukaryotic cells. Int J Nanomedicine. 2013; 8:4033-4042.

178. Reimertz C, Kogel D, Rami A, Chittenden T and Prehn JH. Gene expression during ER stress-induced apoptosis in neurons: induction of the BH3-only protein Bbc3/PUMA and activation of the mitochondrial apoptosis pathway. $\mathrm{J}$ Cell Biol. 2003; 162(4):587-597.

179. Li J, Lee B and Lee AS. Endoplasmic reticulum stressinduced apoptosis: multiple pathways and activation of p53up-regulated modulator of apoptosis (PUMA) and NOXA by p53. J Biol Chem. 2006; 281(11):7260-7270.

180. Leon R, Bhagavatula N, Ulukpo O, McCollum M and Wei J. BimEL as a possible molecular link between proteasome dysfunction and cell death induced by mutant huntingtin. Eur J Neurosci. 2010; 31(11):1915-1925.
181. Ghosh AP, Klocke BJ, Ballestas ME and Roth KA. CHOP potentially co-operates with FOXO3a in neuronal cells to regulate PUMA and BIM expression in response to ER stress. PLoS One. 2012; 7(6):e39586.

182. Galehdar Z, Swan P, Fuerth B, Callaghan SM, Park DS and Cregan SP. Neuronal apoptosis induced by endoplasmic reticulum stress is regulated by ATF4-CHOP-mediated induction of the Bcl-2 homology 3-only member PUMA. J Neurosci. 2010; 30(50):16938-16948.

183. Barthson J, Germano CM, Moore F, Maida A, Drucker DJ, Marchetti P, Gysemans C, Mathieu C, Nunez G, Jurisicova A, Eizirik DL and Gurzov EN. Cytokines tumor necrosis factor-alpha and interferon-gamma induce pancreatic beta-cell apoptosis through STAT1-mediated Bim protein activation. J Biol Chem. 2011; 286(45):39632-39643.

184. Marques-Fernandez F, Planells-Ferrer L, Gozzelino R, Galenkamp KM, Reix S, Llecha-Cano N, Lopez-Soriano J, Yuste VJ, Moubarak RS and Comella JX. TNFalpha induces survival through the FLIP-L-dependent activation of the MAPK/ERK pathway. Cell Death Dis. 2013; 4:e493.

185. Eizirik DL, Moore F, Flamez D and Ortis F. Use of a systems biology approach to understand pancreatic beta-cell death in Type 1 diabetes. Biochem Soc Trans. 2008; 36(Pt 3):321-327.

186. Kim S, Kim HS, Chung KW, Oh SH, Yun JW, Im SH, Lee MK, Kim KW and Lee MS. Essential role for signal transducer and activator of transcription-1 in pancreatic beta-cell death and autoimmune type 1 diabetes of nonobese diabetic mice. Diabetes. 2007; 56(10):2561-2568.

187. Consortium WTCC. Genome-wide association study of 14,000 cases of seven common diseases and 3,000 shared controls. Nature. 2007; 447(7145):661-678.

188. Santin I, Moore F, Colli ML, Gurzov EN, Marselli L, Marchetti P and Eizirik DL. PTPN2, a candidate gene for type 1 diabetes, modulates pancreatic beta-cell apoptosis via regulation of the BH3-only protein Bim. Diabetes. 2011; 60(12):3279-3288.

189. Shin ES, Huang Q, Gurel Z, Palenski TL, Zaitoun I, Sorenson CM and Sheibani N. STAT1-mediated Bim expression promotes the apoptosis of retinal pericytes under high glucose conditions. Cell Death Dis. 2014; 5:e986.

190. Gowda A, Roda J, Hussain SR, Ramanunni A, Joshi T, Schmidt S, Zhang X, Lehman A, Jarjoura D, Carson WE, Kindsvogel W, Cheney C, Caligiuri MA, Tridandapani $\mathrm{S}$, Muthusamy N and Byrd JC. IL-21 mediates apoptosis through up-regulation of the $\mathrm{BH} 3$ family member BIM and enhances both direct and antibody-dependent cellular cytotoxicity in primary chronic lymphocytic leukemia cells in vitro. Blood. 2008; 111(9):4723-4730.

191. Muthalagu N, Junttila MR, Wiese KE, Wolf E, Morton J, Bauer B, Evan GI, Eilers M and Murphy DJ. BIM is the primary mediator of MYC-induced apoptosis in multiple solid tissues. Cell Rep. 2014; 8(5):1347-1353.

192. Campone M, Noel B, Couriaud C, Grau M, Guillemin Y, 
Gautier F, Gouraud W, Charbonnel C, Campion L, Jezequel P, Braun F, Barre B, Coqueret O, Barille-Nion S and Juin P. c-Myc dependent expression of pro-apoptotic Bim renders HER2-overexpressing breast cancer cells dependent on antiapoptotic Mcl-1. Mol Cancer. 2011; 10:110.

193. Hemann MT, Bric A, Teruya-Feldstein J, Herbst A, Nilsson JA, Cordon-Cardo C, Cleveland JL, Tansey WP and Lowe SW. Evasion of the p53 tumour surveillance network by tumour-derived MYC mutants. Nature. 2005; 436(7052):807-811.

194. Ausserlechner MJ, Obexer P, Bock G, Geley S and Kofler R. Cyclin D3 and c-MYC control glucocorticoid-induced cell cycle arrest but not apoptosis in lymphoblastic leukemia cells. Cell Death Differ. 2004; 11(2):165-174.

195. Loffler M, Ausserlechner MJ, Tonko M, Hartmann BL, Bernhard D, Geley S, Helmberg A and Kofler R. c-Myc does not prevent glucocorticoid-induced apoptosis of human leukemic lymphoblasts. Oncogene. 1999; 18(32):46264631.

196. Medh RD, Wang A, Zhou F and Thompson EB. Constitutive expression of ectopic c-Myc delays glucocorticoid-evoked apoptosis of human leukemic CEM-C7 cells. Oncogene. 2001; 20(34):4629-4639.

197. Zhou F, Medh RD and Thompson EB. Glucocorticoid mediated transcriptional repression of c-myc in apoptotic human leukemic CEM cells. J Steroid Biochem Mol Biol. 2000; 73(5):195-202.

198. Thompson EB. Stepping stones in the path of glucocorticoid-driven apoptosis of lymphoid cells. Acta Biochim Biophys Sin (Shanghai). 2008; 40(7):595-600.

199. Sionov RV, Kfir-Erenfeld S, Spokoini R and Yefenof E. A role for bcl-2 in notch1-dependent transcription in thymic lymphoma cells. Adv Hematol. 2012; 2012:435241.

200. Li Y, Choi PS, Casey SC, Dill DL and Felsher DW. MYC through miR-17-92 suppresses specific target genes to maintain survival, autonomous proliferation, and a neoplastic state. Cancer Cell. 2014; 26(2):262-272.

201. Chen L, Li C, Zhang R, Gao X, Qu X, Zhao M, Qiao C, $\mathrm{Xu} \mathrm{J}$ and Li J. miR-17-92 cluster microRNAs confers tumorigenicity in multiple myeloma. Cancer Lett. 2011; 309(1):62-70.

202. Aguda BD, Kim Y, Piper-Hunter MG, Friedman A and Marsh CB. MicroRNA regulation of a cancer network: consequences of the feedback loops involving miR17-92, E2F, and Myc. Proc Natl Acad Sci U S A. 2008; 105(50):19678-19683.

203. Horiuchi D, Kusdra L, Huskey NE, Chandriani S, Lenburg ME, Gonzalez-Angulo AM, Creasman KJ, Bazarov AV, Smyth JW, Davis SE, Yaswen P, Mills GB, Esserman LJ and Goga A. MYC pathway activation in triple-negative breast cancer is synthetic lethal with CDK inhibition. J Exp Med. 2012; 209(4):679-696.

204. Kohler UA, Kurinna S, Schwitter D, Marti A, Schafer M, Hellerbrand C, Speicher T and Werner S. Activated Nrf2 impairs liver regeneration in mice by activation of genes involved in cell-cycle control and apoptosis. Hepatology. 2014; 60(2):670-678.

205. Potluri V, Noothi SK, Vallabhapurapu SD, Yoon SO, Driscoll JJ, Lawrie $\mathrm{CH}$ and Vallabhapurapu S. Transcriptional repression of Bim by a novel YY1-RelA complex is essential for the survival and growth of Multiple Myeloma. PLoS One. 2013; 8(7):e66121.

206. Inta I, Paxian S, Maegele I, Zhang W, Pizzi M, Spano P, Sarnico I, Muhammad S, Herrmann O, Inta D, Baumann B, Liou HC, Schmid RM and Schwaninger M. Bim and Noxa are candidates to mediate the deleterious effect of the NF-kappa B subunit RelA in cerebral ischemia. J Neurosci. 2006; 26(50):12896-12903.

207. Salmanidis M, Brumatti G, Narayan N, Green BD, van den Bergen JA, Sandow JJ, Bert AG, Silke N, Sladic R, Puthalakath H, Rohrbeck L, Okamoto T, Bouillet P, Herold MJ, Goodall GJ, Jabbour AM, et al. Hoxb8 regulates expression of microRNAs to control cell death and differentiation. Cell Death Differ. 2013; 20(10):1370-1380.

208. Ridinger-Saison M, Evanno E, Gallais I, Rimmele P, Selimoglu-Buet D, Sapharikas E, Moreau-Gachelin F and Guillouf C. Epigenetic silencing of Bim transcription by Spi-1/PU.1 promotes apoptosis resistance in leukaemia. Cell Death Differ. 2013; 20(9):1268-1278.

209. Wang E, Kawaoka S, Roe JS, Shi J, Hohmann AF, Xu Y, Bhagwat AS, Suzuki Y, Kinney JB and Vakoc CR. The transcriptional cofactor TRIM33 prevents apoptosis in B lymphoblastic leukemia by deactivating a single enhancer. Elife. 2015;8; 4:e6377.

210. Chen K, Tu Y, Zhang Y, Blair HC, Zhang L and Wu C. PINCH-1 regulates the ERK-Bim pathway and contributes to apoptosis resistance in cancer cells. J Biol Chem. 2008; 283(5):2508-2517.

211. Maeda T, Merghoub T, Hobbs RM, Dong L, Maeda M, Zakrzewski J, van den Brink MR, Zelent A, Shigematsu H, Akashi K, Teruya-Feldstein J, Cattoretti G and Pandolfi PP. Regulation of B versus T lymphoid lineage fate decision by the proto-oncogene LRF. Science. 2007; 316(5826):860866.

212. Maeda T, Ito K, Merghoub T, Poliseno L, Hobbs RM, Wang G, Dong L, Maeda M, Dore LC, Zelent A, Luzzatto L, Teruya-Feldstein J, Weiss MJ and Pandolfi PP. LRF is an essential downstream target of GATA1 in erythroid development and regulates BIM-dependent apoptosis. Dev Cell. 2009; 17(4):527-540.

213. Sankpal UT, Maliakal P, Bose D, Kayaleh O, Buchholz D and Basha R. Expression of specificity protein transcription factors in pancreatic cancer and their association in prognosis and therapy. Curr Med Chem. 2012; 19(22):37793786.

214. Luo J, Wang X, Xia Z, Yang L, Ding Z, Chen S, Lai B and Zhang N. Transcriptional factor specificity protein 1 (SP1) promotes the proliferation of glioma cells by up-regulating midkine (MDK). Mol Biol Cell. 2015; 26(3):430-439. 
215. Lee HE, Choi ES, Jung JY, You MJ, Kim LH and Cho SD. Inhibition of specificity protein 1 by dibenzylideneacetone, a curcumin analogue, induces apoptosis in mucoepidermoid carcinomas and tumor xenografts through Bim and truncated Bid. Oral Oncol. 2014; 50(3):189-195.

216. Choi KH, Shim JH, Huong LD, Cho NP and Cho SD. Inhibition of myeloid cell leukemia-1 by tolfenamic acid induces apoptosis in mucoepidermoid carcinoma. Oral Dis. 2011; 17(5):469-475.

217. Patel AJ, Liao CP, Chen Z, Liu C, Wang Y and Le LQ. BET bromodomain inhibition triggers apoptosis of NF1associated malignant peripheral nerve sheath tumors through Bim induction. Cell Rep. 2014; 6(1):81-92.

218. Fiskus W, Sharma S, Qi J, Valenta JA, Schaub LJ, Shah B, Peth K, Portier BP, Rodriguez M, Devaraj SG, Zhan M, Sheng J, Iyer SP, Bradner JE and Bhalla KN. Highly active combination of BRD4 antagonist and histone deacetylase inhibitor against human acute myelogenous leukemia cells. Mol Cancer Ther. 2014; 13(5):1142-1154.

219. Gallagher SJ, Mijatov B, Gunatilake D, Tiffen JC, Gowrishankar K, Jin L, Pupo GM, Cullinane C, Prinjha RK, Smithers N, McArthur GA, Rizos H and Hersey P. The epigenetic regulator I-BET151 induces BIM-dependent apoptosis and cell cycle arrest of human melanoma cells. J Invest Dermatol. 2014; 134(11):2795-2805.

220. Fiskus W, Sharma S, Qi J, Shah B, Devaraj SG, Leveque C, Portier BP, Iyer S, Bradner JE and Bhalla KN. BET protein antagonist JQ1 is synergistically lethal with FLT3 tyrosine kinase inhibitor (TKI) and overcomes resistance to FLT3TKI in AML cells expressing FLT-ITD. Mol Cancer Ther. 2014; 13(10):2315-2327.

221. Da Costa D, Agathanggelou A, Perry T, Weston V, Petermann E, Zlatanou A, Oldreive C, Wei W, Stewart G, Longman J, Smith E, Kearns P, Knapp S and Stankovic T. BET inhibition as a single or combined therapeutic approach in primary paediatric B-precursor acute lymphoblastic leukaemia. Blood Cancer J. 2013; 3:e126.

222. Xie L, Johnson RS and Freeman RS. Inhibition of NGF deprivation-induced death by low oxygen involves suppression of BIMEL and activation of HIF-1. J Cell Biol. 2005; 168(6):911-920.

223. Muhleisen A, Giaisi M, Kohler R, Krammer PH and LiWeber M. Tax contributes apoptosis resistance to HTLV-1infected T cells via suppression of Bid and Bim expression. Cell Death Dis. 2014; 5:e1575.

224. Whelan KA, Schwab LP, Karakashev SV, Franchetti L, Johannes GJ, Seagroves TN and Reginato MJ. The oncogene HER2/neu (ERBB2) requires the hypoxiainducible factor HIF-1 for mammary tumor growth and anoikis resistance. J Biol Chem. 2013; 288(22):1586515877.

225. Anderton E, Yee J, Smith P, Crook T, White RE and Allday MJ. Two Epstein-Barr virus (EBV) oncoproteins cooperate to repress expression of the proapoptotic tumour-suppressor Bim: clues to the pathogenesis of Burkitt's lymphoma.
Oncogene. 2008; 27(4):421-433.

226. Paschos K, Parker GA, Watanatanasup E, White RE and Allday MJ. BIM promoter directly targeted by EBNA3C in polycomb-mediated repression by EBV. Nucleic Acids Res. 2012; 40(15):7233-7246.

227. McClellan MJ, Wood CD, Ojeniyi O, Cooper TJ, Kanhere A, Arvey A, Webb HM, Palermo RD, Harth-Hertle ML, Kempkes B, Jenner RG and West MJ. Modulation of enhancer looping and differential gene targeting by Epstein-Barr virus transcription factors directs cellular reprogramming. PLoS Pathog. 2013; 9(9):e1003636.

228. Adachi M, Zhao X and Imai K. Nomenclature of dynein light chain-linked $\mathrm{BH} 3$-only protein Bim isoforms. Cell Death Differ. 2005; 12(2):192-193.

229. Anczukow O, Rosenberg AZ, Akerman M, Das S, Zhan L, Karni R, Muthuswamy SK and Krainer AR. The splicing factor SRSF1 regulates apoptosis and proliferation to promote mammary epithelial cell transformation. Nat Struct Mol Biol. 2012; 19(2):220-228.

230. Das S, Anczukow O, Akerman M and Krainer AR. Oncogenic splicing factor SRSF1 is a critical transcriptional target of MYC. Cell Rep. 2012; 1(2):110-117.

231. Li X, Wang J and Manley JL. Loss of splicing factor ASF/ SF2 induces G2 cell cycle arrest and apoptosis, but inhibits internucleosomal DNA fragmentation. Genes Dev. 2005; 19(22):2705-2714.

232. Leu S, Lin YM, Wu CH and Ouyang P. Loss of Pnn expression results in mouse early embryonic lethality and cellular apoptosis through SRSF1-mediated alternative expression of Bcl-xS and ICAD. J Cell Sci. 2012; 125(Pt 13):3164-3172.

233. Nogueira TC, Paula FM, Villate O, Colli ML, Moura RF, Cunha DA, Marselli L, Marchetti P, Cnop M, Julier C and Eizirik DL. GLIS3, a susceptibility gene for type 1 and type 2 diabetes, modulates pancreatic beta cell apoptosis via regulation of a splice variant of the $\mathrm{BH} 3$-only protein Bim. PLoS Genet. 2013; 9(5):e1003532.

234. Eizirik DL, Sammeth M, Bouckenooghe T, Bottu G, Sisino G, Igoillo-Esteve M, Ortis F, Santin I, Colli ML, Barthson J, Bouwens L, Hughes L, Gregory L, Lunter G, Marselli L, Marchetti $\mathrm{P}$, et al. The human pancreatic islet transcriptome: expression of candidate genes for type 1 diabetes and the impact of pro-inflammatory cytokines. PLoS Genet. 2012; 8(3):e1002552.

235. Jiang CC, Lai F, Tay KH, Croft A, Rizos H, Becker TM, Yang F, Liu H, Thorne RF, Hersey P and Zhang XD. Apoptosis of human melanoma cells induced by inhibition of B-RAFV600E involves preferential splicing of bimS. Cell Death Dis. 2010; 1:e69.

236. Hara H, Takeda T, Yamamoto N, Furuya K, Hirose K, Kamiya $\mathrm{T}$ and Adachi T. Zinc-induced modulation of SRSF6 activity alters Bim splicing to promote generation of the most potent apoptotic isoform BimS. FEBS J. 2013; 280(14):3313-3327. 
237. Juan WC, Roca X and Ong ST. Identification of cis-acting elements and splicing factors involved in the regulation of BIM Pre-mRNA splicing. PLoS One. 2014; 9(4):e95210.

238. Batsche E, Yaniv M and Muchardt C. The human SWI/SNF subunit Brm is a regulator of alternative splicing. Nat Struct Mol Biol. 2006; 13(1):22-29.

239. Kahali B, Yu J, Marquez SB, Thompson KW, Liang SY, Lu $\mathrm{L}$ and Reisman D. The silencing of the SWI/SNF subunit and anticancer gene BRM in Rhabdoid tumors. Oncotarget. 2014; 5(10):3316-3332.

240. Fandy TE, Shankar S, Ross DD, Sausville E and Srivastava RK. Interactive effects of HDAC inhibitors and TRAIL on apoptosis are associated with changes in mitochondrial functions and expressions of cell cycle regulatory genes in multiple myeloma. Neoplasia. 2005; 7(7):646-657.

241. Fiskus W, Pranpat M, Bali P, Balasis M, Kumaraswamy S, Boyapalle S, Rocha K, Wu J, Giles F, Manley PW, Atadja P and Bhalla K. Combined effects of novel tyrosine kinase inhibitor AMN107 and histone deacetylase inhibitor LBH589 against Bcr-Abl-expressing human leukemia cells. Blood. 2006; 108(2):645-652.

242. Zhang XD, Gillespie SK, Borrow JM and Hersey P. The histone deacetylase inhibitor suberic bishydroxamate regulates the expression of multiple apoptotic mediators and induces mitochondria-dependent apoptosis of melanoma cells. Mol Cancer Ther. 2004; 3(4):425-435.

243. Paschos K, Smith P, Anderton E, Middeldorp JM, White RE and Allday MJ. Epstein-barr virus latency in B cells leads to epigenetic repression and $\mathrm{CpG}$ methylation of the tumour suppressor gene Bim. PLoS Pathog. 2009; 5(6):e1000492.

244. Richter-Larrea JA, Robles EF, Fresquet V, Beltran E, Rullan AJ, Agirre X, Calasanz MJ, Panizo C, Richter JA, Hernandez JM, Roman-Gomez J, Prosper F and MartinezCliment JA. Reversion of epigenetically mediated BIM silencing overcomes chemoresistance in Burkitt lymphoma. Blood. 2010; 116(14):2531-2542.

245. Moore PS, Barbi S, Donadelli M, Costanzo C, Bassi C, Palmieri $M$ and Scarpa A. Gene expression profiling after treatment with the histone deacetylase inhibitor trichostatin A reveals altered expression of both pro- and anti-apoptotic genes in pancreatic adenocarcinoma cells. Biochim Biophys Acta. 2004; 1693(3):167-176.

246. Mestre-Escorihuela C, Rubio-Moscardo F, Richter JA, Siebert R, Climent J, Fresquet V, Beltran E, Agirre X, Marugan I, Marin M, Rosenwald A, Sugimoto KJ, Wheat LM, Karran EL, Garcia JF, Sanchez L, et al. Homozygous deletions localize novel tumor suppressor genes in B-cell lymphomas. Blood. 2007; 109(1):271-280.

247. De Bruyne E, Bos TJ, Schuit F, Van Valckenborgh E, Menu E, Thorrez L, Atadja P, Jernberg-Wiklund $\mathrm{H}$ and Vanderkerken K. IGF-1 suppresses Bim expression in multiple myeloma via epigenetic and posttranslational mechanisms. Blood. 2010; 115(12):2430-2440.

248. San Jose-Eneriz E, Agirre X, Jimenez-Velasco A, Cordeu
L, Martin V, Arqueros V, Garate L, Fresquet V, Cervantes F, Martinez-Climent JA, Heiniger A, Torres A, Prosper F and Roman-Gomez J. Epigenetic down-regulation of BIM expression is associated with reduced optimal responses to imatinib treatment in chronic myeloid leukaemia. Eur J Cancer. 2009; 45(10):1877-1889.

249. Dong Y, Xiong M, Duan L, Liu Z, Niu T, Luo Y, Wu $\mathrm{X}, \mathrm{Xu} \mathrm{C}$ and $\mathrm{Lu} \mathrm{C}$. H2AX phosphorylation regulated by p38 is involved in Bim expression and apoptosis in chronic myelogenous leukemia cells induced by imatinib. Apoptosis. 2014; 19(8):1281-1292.

250. Stiff T, O'Driscoll M, Rief N, Iwabuchi K, Lobrich M and Jeggo PA. ATM and DNA-PK function redundantly to phosphorylate $\mathrm{H} 2 \mathrm{AX}$ after exposure to ionizing radiation. Cancer Res. 2004; 64(7):2390-2396.

251. Zhang YJ, Lu CR, Cao Y, Luo Y, Bao RF, Yan S, Xue $\mathrm{M}$, Zhu F, Wang Z and Duan LN. Imatinib induces H2AX phosphorylation and apoptosis in chronic myelogenous leukemia cells in vitro via caspase-3/Mst1 pathway. Acta Pharmacol Sin. 2012; 33(4):551-557.

252. Lu C, Zhu F, Cho YY, Tang F, Zykova T, Ma WY, Bode $\mathrm{AM}$ and Dong Z. Cell apoptosis: requirement of H2AX in DNA ladder formation, but not for the activation of caspase-3. Mol Cell. 2006; 23(1):121-132.

253. Matsui $H$, Asou $H$ and Inaba T. Cytokines direct the regulation of Bim mRNA stability by heat-shock cognate protein 70. Mol Cell. 2007; 25(1):99-112.

254. Terasawa K, Ichimura A, Sato F, Shimizu K and Tsujimoto G. Sustained activation of ERK1/2 by NGF induces microRNA-221 and 222 in PC12 cells. FEBS J. 2009; 276(12):3269-3276.

255. Jia J, Yao P, Arif A and Fox PL. Regulation and dysregulation of 3'UTR-mediated translational control. Curr Opin Genet Dev. 2013; 23(1):29-34.

256. Davila D, Jimenez-Mateos EM, Mooney CM, Velasco G, Henshall DC and Prehn JH. Hsp27 binding to the 3'UTR of bim mRNA prevents neuronal death during oxidative stressinduced injury: a novel cytoprotective mechanism. Mol Biol Cell. 2014; 25(21):3413-3423.

257. Wang X, Tokuda H, Hatakeyama D, Hirade K, Niwa M, Ito $\mathrm{H}$, Kato $\mathrm{K}$ and Kozawa $\mathrm{O}$. Mechanism of simvastatin on induction of heat shock protein in osteoblasts. Arch Biochem Biophys. 2003; 415(1):6-13.

258. Urbich C, Knau A, Fichtlscherer S, Walter DH, Bruhl T, Potente M, Hofmann WK, de Vos S, Zeiher AM and Dimmeler S. FOXO-dependent expression of the proapoptotic protein Bim: pivotal role for apoptosis signaling in endothelial progenitor cells. FASEB J. 2005; 19(8):974-976.

259. Su H, Trombly MI, Chen J and Wang X. Essential and overlapping functions for mammalian Argonautes in microRNA silencing. Genes Dev. 2009; 23(3):304-317.

260. Koralov SB, Muljo SA, Galler GR, Krek A, Chakraborty T, Kanellopoulou C, Jensen K, Cobb BS, Merkenschlager 
M, Rajewsky N and Rajewsky K. Dicer ablation affects antibody diversity and cell survival in the B lymphocyte lineage. Cell. 2008; 132(5):860-874.

261. Wang S, Tang C, Zhang Q and Chen W. Reduced miR-9 and miR-181a expression down-regulates Bim concentration and promote osteoclasts survival. Int J Clin Exp Pathol. 2014; 7(5):2209-2218.

262. Taylor MA, Sossey-Alaoui K, Thompson CL, Danielpour $\mathrm{D}$ and Schiemann WP. TGF-beta upregulates miR-181a expression to promote breast cancer metastasis. J Clin Invest. 2013; 123(1):150-163.

263. Xiao C, Srinivasan L, Calado DP, Patterson HC, Zhang B, Wang J, Henderson JM, Kutok JL and Rajewsky K. Lymphoproliferative disease and autoimmunity in mice with increased miR-17-92 expression in lymphocytes. Nat Immunol. 2008; 9(4):405-414.

264. Ventura A, Young AG, Winslow MM, Lintault L, Meissner A, Erkeland SJ, Newman J, Bronson RT, Crowley D, Stone JR, Jaenisch R, Sharp PA and Jacks T. Targeted deletion reveals essential and overlapping functions of the miR17 through 92 family of miRNA clusters. Cell. 2008; 132(5):875-886.

265. Harada M, Pokrovskaja-Tamm K, Soderhall S, Heyman M, Grander D and Corcoran M. Involvement of miR17 pathway in glucocorticoid-induced cell death in pediatric acute lymphoblastic leukemia. Leuk Lymphoma. 2012; 53(10):2041-2050.

266. Molitoris JK, McColl KS and Distelhorst CW. Glucocorticoid-mediated repression of the oncogenic microRNA cluster miR-17 92 contributes to the induction of Bim and initiation of apoptosis. Mol Endocrinol. 2011; 25(3):409-420.

267. Niu H, Wang K, Zhang A, Yang S, Song Z, Wang W, Qian C, Li X, Zhu Y and Wang Y. miR-92a is a critical regulator of the apoptosis pathway in glioblastoma with inverse expression of BCL2L11. Oncol Rep. 2012; 28(5):17711777.

268. Pernaute B, Spruce T, Smith KM, Sanchez-Nieto JM, Manzanares M, Cobb B and Rodriguez TA. MicroRNAs control the apoptotic threshold in primed pluripotent stem cells through regulation of BIM. Genes Dev. 2014; 28(17):1873-1878.

269. Zhang H, Zuo Z, Lu X, Wang L, Wang H and Zhu Z. MiR25 regulates apoptosis by targeting Bim in human ovarian cancer. Oncol Rep. 2012; 27(2):594-598.

270. Kan T, Sato F, Ito T, Matsumura N, David S, Cheng Y, Agarwal R, Paun BC, Jin Z, Olaru AV, Selaru FM, Hamilton JP, Yang J, Abraham JM, Mori Y and Meltzer SJ. The miR-106b-25 polycistron, activated by genomic amplification, functions as an oncogene by suppressing p21 and Bim. Gastroenterology. 2009; 136(5):1689-1700.

271. Gupta S, Read DE, Deepti A, Cawley K, Gupta A, Oommen D, Verfaillie T, Matus S, Smith MA, Mott JL, Agostinis P, Hetz $\mathrm{C}$ and Samali A. Perk-dependent repression of miR- 106b-25 cluster is required for ER stress-induced apoptosis. Cell Death Dis. 2012; 3:e333.

272. Zhao ZN, Bai JX, Zhou Q, Yan B, Qin WW, Jia LT, Meng YL, Jin BQ, Yao LB, Wang T and Yang AG. TSA suppresses miR-106b-93-25 cluster expression through downregulation of MYC and inhibits proliferation and induces apoptosis in human EMC. PLoS One. 2012; 7(9):e45133.

273. Sahin F and Sladek TL. E2F-1 has dual roles depending on the cell cycle. Int J Biol Sci. 2010; 6(2):116-128.

274. Haftmann C, Stittrich AB, Zimmermann J, Fang Z, Hradilkova K, Bardua M, Westendorf K, Heinz GA, Riedel R, Siede J, Lehmann K, Weinberger EE, Zimmel D, Lauer U, Haupl T, Sieper J, et al. miR-148a is upregulated by Twist1 and T-bet and promotes Th1-cell survival by regulating the proapoptotic gene Bim. Eur J Immunol. 2015; 45(4):1192-1205.

275. Kim J, Zhang Y, Skalski M, Hayes J, Kefas B, Schiff D, Purow B, Parsons S, Lawler S and Abounader R. microRNA-148a is a prognostic oncomiR that targets MIG6 and BIM to regulate EGFR and apoptosis in glioblastoma. Cancer Res. 2014; 74(5):1541-1553.

276. Gocek E, Wang X, Liu X, Liu CG and Studzinski GP. MicroRNA-32 upregulation by 1,25-dihydroxyvitamin D3 in human myeloid leukemia cells leads to Bim targeting and inhibition of AraC-induced apoptosis. Cancer Res. 2011; 71(19):6230-6239.

277. Ambs S, Prueitt RL, Yi M, Hudson RS, Howe TM, Petrocca F, Wallace TA, Liu CG, Volinia S, Calin GA, Yfantis HG, Stephens RM and Croce CM. Genomic profiling of microRNA and messenger RNA reveals deregulated microRNA expression in prostate cancer. Cancer Res. 2008; 68(15):6162-6170.

278. Chen Z, Chen LY, Dai HY, Wang P, Gao S and Wang K. miR-301a promotes pancreatic cancer cell proliferation by directly inhibiting Bim expression. J Cell Biochem. 2012; 113(10):3229-3235.

279. Floyd DH, Zhang Y, Dey BK, Kefas B, Breit H, Marks K, Dutta A, Herold-Mende C, Synowitz M, Glass R, Abounader R and Purow BW. Novel anti-apoptotic microRNAs 582-5p and 363 promote human glioblastoma stem cell survival via direct inhibition of caspase 3 , caspase 9, and Bim. PLoS One. 2014; 9(5):e96239.

280. Wang H, Ye Y, Zhu Z, Mo L, Lin C, Wang Q, Gong X, He $\mathrm{X}, \mathrm{Lu}$ G, Lu F and Zhang S. MiR-124 Regulates Apoptosis and Autophagy Process in MPTP Model of Parkinson's Disease by Targeting to Bim. Brain Pathol. 2015 May 15 . doi: 10.1111/bpa.12267. [Epub ahead of print].

281. Qian L, Van Laake LW, Huang Y, Liu S, Wendland MF and Srivastava D. miR-24 inhibits apoptosis and represses Bim in mouse cardiomyocytes. J Exp Med. 2011; 208(3):549560.

282. Yang A, Ma J, Wu M, Qin W, Zhao B, Shi Y, Jin Y and Xie Y. Aberrant microRNA-182 expression is associated with 
glucocorticoid resistance in lymphoblastic malignancies. Leuk Lymphoma. 2012; 53(12):2465-2473.

283. Rouquette-Jazdanian AK, Kortum RL, Li W, Merrill RK, Nguyen PH, Samelson LE and Sommers CL. miR155 Controls Lymphoproliferation in LAT Mutant Mice by Restraining T-Cell Apoptosis via SHIP-1/mTOR and PAK1/FOXO3/BIM Pathways. PLoS One. 2015; 10(6):e0131823.

284. O'Connell RM, Chaudhuri AA, Rao DS and Baltimore D. Inositol phosphatase SHIP1 is a primary target of miR-155. Proc Natl Acad Sci U S A. 2009; 106(17):7113-7118.

285. Zhang JG, Guo JF, Liu DL, Liu Q and Wang JJ. MicroRNA-101 exerts tumor-suppressive functions in nonsmall cell lung cancer through directly targeting enhancer of zeste homolog 2. J Thorac Oncol. 2011; 6(4):671-678.

286. Garofalo M, Quintavalle C, Romano G, Croce CM and Condorelli G. miR221/222 in cancer: their role in tumor progression and response to therapy. Curr Mol Med. 2012; 12(1):27-33

287. Calin GA, Dumitru CD, Shimizu M, Bichi R, Zupo S, Noch E, Aldler H, Rattan S, Keating M, Rai K, Rassenti L, Kipps T, Negrini M, Bullrich F and Croce CM. Frequent deletions and down-regulation of micro- RNA genes miR15 and miR16 at 13q14 in chronic lymphocytic leukemia. Proc Natl Acad Sci U S A. 2002; 99(24):15524-15529.

288. Wang H, Li J, Chi H, Zhang F, Zhu X, Cai J and Yang X. MicroRNA-181c targets Bcl-2 and regulates mitochondrial morphology in myocardial cells. J Cell Mol Med. 2015; 19(9):2084-97.

289. Lv B, Liu Z, Wang S, Liu F, Yang X, Hou J, Hou Z and Chen B. MiR-29a promotes intestinal epithelial apoptosis in ulcerative colitis by down-regulating Mcl-1. Int J Clin Exp Pathol. 2014; 7(12):8542-8552.

290. Zarogoulidis P, Petanidis S, Kioseoglou E, Domvri K, Anestakis D and Zarogoulidis K. MiR-205 and miR-218 expression is associated with carboplatin chemoresistance and regulation of apoptosis via Mcl-1 and Survivin in lung cancer cells. Cell Signal. 2015; 27(8):1576-1588.

291. Konno Y, Dong P, Xiong Y, Suzuki F, Lu J, Cai M, Watari H, Mitamura T, Hosaka M, Hanley SJ, Kudo M and Sakuragi N. MicroRNA-101 targets EZH2, MCL-1 and FOS to suppress proliferation, invasion and stem celllike phenotype of aggressive endometrial cancer cells. Oncotarget. 2014; 5(15):6049-6062.

292. Mao K, Zhang J, He C, Xu K, Liu J, Sun J, Wu G, Tan C, Zeng Y, Wang J and Xiao Z. Restoration of miR-193b sensitizes Hepatitis B virus-associated hepatocellular carcinoma to sorafenib. Cancer Lett. 2014; 352(2):245-252.

293. Wang L, Ye X, Liu Y, Wei W and Wang Z. Aberrant regulation of FBW7 in cancer. Oncotarget. 2014; 5(8):20002015.

294. Hales EC, Taub JW and Matherly LH. New insights into Notch1 regulation of the PI3K-AKT-mTOR1 signaling axis: targeted therapy of gamma-secretase inhibitor resistant
T-cell acute lymphoblastic leukemia. Cell Signal. 2014; 26(1):149-161.

295. Hubner A, Barrett T, Flavell RA and Davis RJ. Multisite phosphorylation regulates Bim stability and apoptotic activity. Mol Cell. 2008; 30(4):415-425.

296. Ley R, Balmanno K, Hadfield K, Weston C and Cook SJ. Activation of the ERK1/2 signaling pathway promotes phosphorylation and proteasome-dependent degradation of the BH3-only protein, Bim. J Biol Chem. 2003; 278(21):18811-18816.

297. Luciano F, Jacquel A, Colosetti P, Herrant M, Cagnol S, Pages $G$ and Auberger P. Phosphorylation of Bim-EL by Erk1/2 on serine 69 promotes its degradation via the proteasome pathway and regulates its proapoptotic function. Oncogene. 2003; 22(43):6785-6793.

298. Wiggins CM, Tsvetkov P, Johnson M, Joyce CL, Lamb CA, Bryant NJ, Komander D, Shaul Y and Cook SJ. BIM(EL), an intrinsically disordered protein, is degraded by $20 \mathrm{~S}$ proteasomes in the absence of poly-ubiquitylation. J Cell Sci. 2011; 124(Pt 6):969-977.

299. Hinds MG, Smits C, Fredericks-Short R, Risk JM, Bailey M, Huang DC and Day CL. Bim, Bad and Bmf: intrinsically unstructured $\mathrm{BH} 3$-only proteins that undergo a localized conformational change upon binding to prosurvival Bcl-2 targets. Cell Death Differ. 2007; 14(1):128-136.

300. Ley R, Ewings KE, Hadfield K, Howes E, Balmanno K and Cook SJ. Extracellular signal-regulated kinases 1/2 are serum-stimulated "Bim(EL) kinases" that bind to the BH3-only protein Bim(EL) causing its phosphorylation and turnover. J Biol Chem. 2004; 279(10):8837-8847.

301. Ewings KE, Wiggins CM and Cook SJ. Bim and the prosurvival Bcl-2 proteins: opposites attract, ERK repels. Cell Cycle. 2007; 6(18):2236-2240.

302. Ewings KE, Hadfield-Moorhouse K, Wiggins CM, Wickenden JA, Balmanno K, Gilley R, Degenhardt K, White E and Cook SJ. ERK1/2-dependent phosphorylation of BimEL promotes its rapid dissociation from Mcl-1 and Bcl-xL. EMBO J. 2007; 26(12):2856-2867.

303. Czabotar PE, Lee EF, van Delft MF, Day CL, Smith BJ, Huang DC, Fairlie WD, Hinds MG and Colman PM. Structural insights into the degradation of Mcl-1 induced by BH3 domains. Proc Natl Acad Sci U S A. 2007; 104(15):6217-6222.

304. Kuroda J, Puthalakath H, Cragg MS, Kelly PN, Bouillet P, Huang DC, Kimura S, Ottmann OG, Druker BJ, Villunger A, Roberts AW and Strasser A. Bim and Bad mediate imatinib-induced killing of Bcr/Abl+ leukemic cells, and resistance due to their loss is overcome by a $\mathrm{BH} 3$ mimetic. Proc Natl Acad Sci U S A. 2006; 103(40):14907-14912.

305. Aichberger KJ, Mayerhofer M, Krauth MT, Vales A, Kondo R, Derdak S, Pickl WF, Selzer E, Deininger M, Druker BJ, Sillaber C, Esterbauer H and Valent P. Lowlevel expression of proapoptotic Bcl-2-interacting mediator in leukemic cells in patients with chronic myeloid leukemia: 
role of $\mathrm{BCR} / \mathrm{ABL}$, characterization of underlying signaling pathways, and reexpression by novel pharmacologic compounds. Cancer Res. 2005; 65(20):9436-9444.

306. Cragg MS, Kuroda J, Puthalakath H, Huang DC and Strasser A. Gefitinib-induced killing of NSCLC cell lines expressing mutant EGFR requires BIM and can be enhanced by BH3 mimetics. PLoS Med. 2007; 4(10):16811689; discussion 1690.

307. Costa DB, Halmos B, Kumar A, Schumer ST, Huberman MS, Boggon TJ, Tenen DG and Kobayashi S. BIM mediates EGFR tyrosine kinase inhibitor-induced apoptosis in lung cancers with oncogenic EGFR mutations. PLoS Med. 2007; 4(10):1669-1679; discussion 1680.

308. Gong Y, Somwar R, Politi K, Balak M, Chmielecki J, Jiang $\mathrm{X}$ and Pao W. Induction of BIM is essential for apoptosis triggered by EGFR kinase inhibitors in mutant EGFR-dependent lung adenocarcinomas. PLoS Med. 2007; 4(10):e294.

309. Deng J, Shimamura T, Perera S, Carlson NE, Cai D, Shapiro GI, Wong KK and Letai A. Proapoptotic BH3only BCL-2 family protein BIM connects death signaling from epidermal growth factor receptor inhibition to the mitochondrion. Cancer Res. 2007; 67(24):11867-11875.

310. Faber AC, Li D, Song Y, Liang MC, Yeap BY, Bronson RT, Lifshits E, Chen Z, Maira SM, Garcia-Echeverria C, Wong KK and Engelman JA. Differential induction of apoptosis in HER2 and EGFR addicted cancers following PI3K inhibition. Proc Natl Acad Sci U S A. 2009; 106(46):1950319508.

311. Biswas SC and Greene LA. Nerve growth factor (NGF) down-regulates the Bcl-2 homology 3 (BH3) domain-only protein Bim and suppresses its proapoptotic activity by phosphorylation. J Biol Chem. 2002; 277(51):49511-49516.

312. Dehan E, Bassermann F, Guardavaccaro D, VasiliverShamis G, Cohen M, Lowes KN, Dustin M, Huang DC, Taunton J and Pagano M. betaTrCP- and Rsk1/2-mediated degradation of BimEL inhibits apoptosis. Mol Cell. 2009; 33(1):109-116.

313. Wykosky J, Hu J, Gomez GG, Taylor T, Villa GR, Pizzo D, VandenBerg SR, Thorne AH, Chen CC, Mischel PS, Gonias SL, Cavenee WK and Furnari FB. A urokinase receptor-Bim signaling axis emerges during EGFR inhibitor resistance in mutant EGFR glioblastoma. Cancer Res. 2015; 75(2):394-404.

314. Wickenden JA, Jin H, Johnson M, Gillings AS, Newson C, Austin M, Chell SD, Balmanno K, Pritchard CA and Cook SJ. Colorectal cancer cells with the BRAF(V600E) mutation are addicted to the ERK1/2 pathway for growth factor-independent survival and repression of BIM. Oncogene. 2008; 27(57):7150-7161.

315. Gillings AS, Balmanno K, Wiggins CM, Johnson M and Cook SJ. Apoptosis and autophagy: BIM as a mediator of tumour cell death in response to oncogene-targeted therapeutics. FEBS J. 2009; 276(21):6050-6062.
316. Rambal AA, Panaguiton ZL, Kramer L, Grant S and Harada H. MEK inhibitors potentiate dexamethasone lethality in acute lymphoblastic leukemia cells through the proapoptotic molecule BIM. Leukemia. 2009; 23(10):17441754.

317. Rahmani M, Anderson A, Habibi JR, Crabtree TR, Mayo M, Harada H, Ferreira-Gonzalez A, Dent P and Grant S. The $\mathrm{BH} 3$-only protein Bim plays a critical role in leukemia cell death triggered by concomitant inhibition of the PI3K/Akt and MEK/ERK1/2 pathways. Blood. 2009; 114(20):45074516.

318. Park EJ, Woo SM, Min KJ and Kwon TK. Transcriptional and post-translational regulation of Bim controls apoptosis in melatonin-treated human renal cancer Caki cells. J Pineal Res. 2014; 56(1):97-106.

319. Vriend J and Reiter RJ. Melatonin as a proteasome inhibitor. Is there any clinical evidence? Life Sci. 2014; 115(1-2):814.

320. Carbajo-Pescador S, Steinmetz C, Kashyap A, Lorenz S, Mauriz JL, Heise M, Galle PR, Gonzalez-Gallego J and Strand S. Melatonin induces transcriptional regulation of Bim by FoxO3a in HepG2 cells. Br J Cancer. 2013; 108(2):442-449.

321. Okuno S, Saito A, Hayashi T and Chan PH. The c-Jun $\mathrm{N}$-terminal protein kinase signaling pathway mediates Bax activation and subsequent neuronal apoptosis through interaction with Bim after transient focal cerebral ischemia. J Neurosci. 2004; 24(36):7879-7887.

322. Akiyama T, Dass CR and Choong PF. Bim-targeted cancer therapy: a link between drug action and underlying molecular changes. Mol Cancer Ther. 2009; 8(12):31733180.

323. Becker EB and Bonni A. Pin1 mediates neural-specific activation of the mitochondrial apoptotic machinery. Neuron. 2006; 49(5):655-662.

324. Becker EB, Howell J, Kodama Y, Barker PA and Bonni A. Characterization of the c-Jun N-terminal kinase-BimEL signaling pathway in neuronal apoptosis. J Neurosci. 2004; 24(40):8762-8770.

325. Reginato MJ, Mills KR, Becker EB, Lynch DK, Bonni A, Muthuswamy SK and Brugge JS. Bim regulation of lumen formation in cultured mammary epithelial acini is targeted by oncogenes. Mol Cell Biol. 2005; 25(11):4591-4601.

326. Leung KT, Li KK, Sun SS, Chan PK, Ooi VE and Chiu LC. Activation of the JNK pathway promotes phosphorylation and degradation of BimEL--a novel mechanism of chemoresistance in T-cell acute lymphoblastic leukemia. Carcinogenesis. 2008; 29(3):544-551.

327. Cai B, Chang SH, Becker EB, Bonni A and Xia Z. p38 MAP kinase mediates apoptosis through phosphorylation of BimEL at Ser-65. J Biol Chem. 2006; 281(35):2521525222.

328. Tzivion G, Dobson $M$ and Ramakrishnan G. FoxO transcription factors; Regulation by AKT and 14-3-3 
proteins. Biochim Biophys Acta. 2011; 1813(11):19381945.

329. Jang SW, Yang SJ, Srinivasan S and Ye K. Akt phosphorylates MstI and prevents its proteolytic activation, blocking FOXO3 phosphorylation and nuclear translocation. J Biol Chem. 2007; 282(42):30836-30844.

330. Lehtinen MK, Yuan Z, Boag PR, Yang Y, Villen J, Becker EB, DiBacco S, de la Iglesia N, Gygi S, Blackwell TK and Bonni A. A conserved MST-FOXO signaling pathway mediates oxidative-stress responses and extends life span. Cell. 2006; 125(5):987-1001.

331. Xiao L, Chen D, Hu P, Wu J, Liu W, Zhao Y, Cao M, Fang Y, Bi W, Zheng Z, Ren J, Ji G, Wang Y and Yuan Z. The c-Abl-MST1 signaling pathway mediates oxidative stressinduced neuronal cell death. J Neurosci. 2011; 31(26):96119619.

332. Atfi A, Abecassis L and Bourgeade MF. Bcr-Abl activates the $\mathrm{AKT} / \mathrm{Fox} \mathrm{O} 3$ signalling pathway to restrict transforming growth factor-beta-mediated cytostatic signals. EMBO Rep. 2005; 6(10):985-991.

333. Morotti A, Panuzzo C, Crivellaro S, Pergolizzi B, Familiari U, Berger AH, Saglio G and Pandolfi PP. BCR-ABL disrupts PTEN nuclear-cytoplasmic shuttling through phosphorylation-dependent activation of HAUSP. Leukemia. 2014; 28(6):1326-1333.

334. Morotti A, Panuzzo C, Crivellaro S, Carra G, Fava C, Guerrasio A, Pandolfi PP and Saglio G. BCR-ABL inactivates cytosolic PTEN through Casein Kinase II mediated tail phosphorylation. Cell Cycle. 2015; 14(7):973979.

335. Hazlehurst LA, Enkemann SA, Beam CA, Argilagos RF, Painter J, Shain KH, Saporta S, Boulware D, Moscinski L, Alsina M and Dalton WS. Genotypic and phenotypic comparisons of de novo and acquired melphalan resistance in an isogenic multiple myeloma cell line model. Cancer Res. 2003; 63(22):7900-7906.

336. Qi XJ, Wildey GM and Howe PH. Evidence that Ser87 of BimEL is phosphorylated by Akt and regulates BimEL apoptotic function. J Biol Chem. 2006; 281(2):813-823.

337. Guan H, Song L, Cai J, Huang Y, Wu J, Yuan J, Li J and Li M. Sphingosine kinase 1 regulates the Akt/FOXO3a/Bim pathway and contributes to apoptosis resistance in glioma cells. PLoS One. 2011; 6(5):e19946.

338. Xiong H, Wang J, Guan H, Wu J, Xu R, Wang M, Rong X, Huang K, Huang J, Liao Q, Fu Y and Yuan J. SphK1 confers resistance to apoptosis in gastric cancer cells by downregulating Bim via stimulating Akt/FoxO3a signaling. Oncol Rep. 2014; 32(4):1369-1373.

339. Rutherford C, Childs S, Ohotski J, McGlynn L, Riddick M, MacFarlane S, Tasker D, Pyne S, Pyne NJ, Edwards J and Palmer TM. Regulation of cell survival by sphingosine-1phosphate receptor S1P1 via reciprocal ERK-dependent suppression of Bim and PI-3-kinase/protein kinase C-mediated upregulation of Mcl-1. Cell Death Dis. 2013; 4:e927.

340. Tatsumi Y, Takano R, Islam MS, Yokochi T, Itami M, Nakamura $\mathrm{Y}$ and Nakagawara A. BMCC1, which is an interacting partner of BCL2, attenuates AKT activity, accompanied by apoptosis. Cell Death Dis. 2015; 6:e1607.

341. Zhang L and Insel PA. The pro-apoptotic protein Bim is a convergence point for $\mathrm{cAMP} /$ protein kinase A- and glucocorticoid-promoted apoptosis of lymphoid cells. J Biol Chem. 2004; 279(20):20858-20865.

342. Zambon AC, Wilderman A, Ho A and Insel PA. Increased expression of the pro-apoptotic protein BIM, a mechanism for cAMP/protein kinase A (PKA)-induced apoptosis of immature T cells. J Biol Chem. 2011; 286(38):3326033267.

343. Moujalled D, Weston R, Anderton H, Ninnis R, Goel P, Coley A, Huang DC, Wu L, Strasser A and Puthalakath H. Cyclic-AMP-dependent protein kinase A regulates apoptosis by stabilizing the $\mathrm{BH} 3$-only protein Bim. EMBO Rep. 2011; 12(1):77-83.

344. Wan L, Tan M, Yang J, Inuzuka H, Dai X, Wu T, Liu J, Shaik S, Chen G, Deng J, Malumbres M, Letai A, Kirschner MW, Sun Y and Wei W. APC(Cdc20) suppresses apoptosis through targeting Bim for ubiquitination and destruction. Dev Cell. 2014; 29(4):377-391.

345. Moustafa-Kamal M, Gamache I, Lu Y, Li S and Teodoro JG. BimEL is phosphorylated at mitosis by Aurora A and targeted for degradation by betaTrCP1. Cell Death Differ. 2013; 20(10):1393-1403.

346. Mac Fhearraigh S and Mc Gee MM. Cyclin B1 interacts with the BH3-only protein Bim and mediates its phosphorylation by Cdk1 during mitosis. Cell Cycle. 2011; 10(22):3886-3896.

347. Gilley R, Lochhead PA, Balmanno K, Oxley D, Clark J and Cook SJ. CDK1, not ERK1/2 or ERK5, is required for mitotic phosphorylation of BIMEL. Cell Signal. 2012; 24(1):170-180.

348. Graos M, Almeida AD and Chatterjee S. Growth-factordependent phosphorylation of Bim in mitosis. Biochem J. 2005; 388(Pt 1):185-194.

349. Kato T, Daigo Y, Aragaki M, Ishikawa K, Sato M and Kaji M. Overexpression of CDC20 predicts poor prognosis in primary non-small cell lung cancer patients. J Surg Oncol. 2012; 106(4):423-430.

350. Haschka MD, Soratroi C, Kirschnek S, Hacker G, Hilbe R, Geley S, Villunger A and Fava LL. The NOXA-MCL1BIM axis defines lifespan on extended mitotic arrest. Nat Commun. 2015; 6:6891.

351. Ness JM, Harvey CA, Strasser A, Bouillet P, Klocke BJ and Roth KA. Selective involvement of BH3-only Bcl-2 family members Bim and Bad in neonatal hypoxia-ischemia. Brain Res. 2006; 1099(1):150-159.

352. Biswas SC, Shi Y, Vonsattel JP, Leung CL, Troy CM and Greene LA. Bim is elevated in Alzheimer's disease neurons and is required for beta-amyloid-induced neuronal 
apoptosis. J Neurosci. 2007; 27(4):893-900.

353. Lauer $\mathrm{C}$, Brunner $\mathrm{T}$ and Corazza N. The proapoptotic Bcl-2 family member Bim plays a central role during the development of virus-induced hepatitis. J Immunol. 2012; 188(2):916-922.

354. Chang KC, Unsinger J, Davis CG, Schwulst SJ, Muenzer JT, Strasser A and Hotchkiss RS. Multiple triggers of cell death in sepsis: death receptor and mitochondrial-mediated apoptosis. FASEB J. 2007; 21(3):708-719.

355. Putcha GV, Moulder KL, Golden JP, Bouillet P, Adams JA, Strasser A and Johnson EM. Induction of BIM, a proapoptotic BH3-only BCL-2 family member, is critical for neuronal apoptosis. Neuron. 2001; 29(3):615-628.

356. Granato A, Hayashi EA, Baptista BJ, Bellio M and Nobrega A. IL-4 regulates Bim expression and promotes B cell maturation in synergy with BAFF conferring resistance to cell death at negative selection checkpoints. J Immunol. 2014; 192(12):5761-5775.

357. Villunger A, Scott C, Bouillet P and Strasser A. Essential role for the $\mathrm{BH} 3$-only protein $\mathrm{Bim}$ but redundant roles for Bax, Bcl-2, and Bcl-w in the control of granulocyte survival. Blood. 2003; 101(6):2393-2400.

358. Alfredsson J, Puthalakath H, Martin H, Strasser A and Nilsson G. Proapoptotic Bcl-2 family member Bim is involved in the control of mast cell survival and is induced together with Bcl-XL upon IgE-receptor activation. Cell Death Differ. 2005; 12(2):136-144.

359. Moller C, Alfredsson J, Engstrom M, Wootz H, Xiang Z, Lennartsson J, Jonsson JI and Nilsson G. Stem cell factor promotes mast cell survival via inactivation of FOXO3amediated transcriptional induction and MEK-regulated phosphorylation of the proapoptotic protein Bim. Blood. 2005; 106(4):1330-1336.

360. Akiyama T, Bouillet P, Miyazaki T, Kadono Y, Chikuda H, Chung UI, Fukuda A, Hikita A, Seto H, Okada T, Inaba T, Sanjay A, Baron R, Kawaguchi H, Oda H, Nakamura K, et al. Regulation of osteoclast apoptosis by ubiquitylation of proapoptotic BH3-only Bcl-2 family member Bim. EMBO J. 2003; 22(24):6653-6664.

361. Weston CR, Balmanno K, Chalmers C, Hadfield K, Molton SA, Ley R, Wagner EF and Cook SJ. Activation of ERK1/2 by deltaRaf-1:ER* represses Bim expression independently of the JNK or PI3K pathways. Oncogene. 2003; 22(9):12811293.

362. Harris CA and Johnson EM, Jr. BH3-only Bcl-2 family members are coordinately regulated by the JNK pathway and require Bax to induce apoptosis in neurons. $\mathrm{J}$ Biol Chem. 2001; 276(41):37754-37760.

363. Besirli CG, Wagner EF and Johnson EM, Jr. The limited role of NH2-terminal c-Jun phosphorylation in neuronal apoptosis: identification of the nuclear pore complex as a potential target of the JNK pathway. J Cell Biol. 2005; 170(3):401-411.

364. Biswas SC, Liu DX and Greene LA. Bim is a direct target of a neuronal E2F-dependent apoptotic pathway. J Neurosci. 2005; 25(37):8349-8358.

365. Li WQ, Guszczynski T, Hixon JA and Durum SK. Interleukin-7 regulates Bim proapoptotic activity in peripheral T-cell survival. Mol Cell Biol. 2010; 30(3):590600.

366. Abutin RM, Chen J, Lung TK, Lloyd JA, Sawyer ST and Harada H. Erythropoietin-induced phosphorylation/ degradation of BIM contributes to survival of erythroid cells. Exp Hematol. 2009; 37(2):151-158.

367. Huntington ND, Puthalakath H, Gunn P, Naik E, Michalak EM, Smyth MJ, Tabarias H, Degli-Esposti MA, Dewson G, Willis SN, Motoyama N, Huang DC, Nutt SL, Tarlinton DM and Strasser A. Interleukin 15-mediated survival of natural killer cells is determined by interactions among Bim, Noxa and Mcl-1. Nat Immunol. 2007; 8(8):856-863.

368. Shenoy AR, Kirschnek S and Hacker G. IL-15 regulates Bcl-2 family members Bim and Mcl-1 through JAK/STAT and PI3K/AKT pathways in T cells. Eur J Immunol. 2014; 44(8):2500-2507.

369. Biswas SC, Buteau J and Greene LA. Glucagon-like peptide-1 (GLP-1) diminishes neuronal degeneration and death caused by NGF deprivation by suppressing Bim induction. Neurochem Res. 2008; 33(9):1845-1851.

370. Yang W, Klaman LD, Chen B, Araki T, Harada H, Thomas SM, George EL and Neel BG. An Shp2/SFK/Ras/Erk signaling pathway controls trophoblast stem cell survival. Dev Cell. 2006; 10(3):317-327.

371. McNamara CA, Sarembock IJ, Gimple LW, Fenton JW, 2nd, Coughlin SR and Owens GK. Thrombin stimulates proliferation of cultured rat aortic smooth muscle cells by a proteolytically activated receptor. J Clin Invest. 1993; 91(1):94-98.

372. Chambard JC, Paris S, L'Allemain G and Pouyssegur J. Two growth factor signalling pathways in fibroblasts distinguished by pertussis toxin. Nature. 1987; 326(6115):800-803.

373. LaMorte VJ, Kennedy ED, Collins LR, Goldstein D, Harootunian AT, Brown JH and Feramisco JR. A requirement for Ras protein function in thrombin-stimulated mitogenesis in astrocytoma cells. J Biol Chem. 1993; 268(26):19411-19415.

374. Patterson C, Stouffer GA, Madamanchi N and Runge MS. New tricks for old dogs: nonthrombotic effects of thrombin in vessel wall biology. Circ Res. 2001; 88(10):987-997.

375. Coughlin SR. Thrombin signalling and protease-activated receptors. Nature. 2000; 407(6801):258-264.

376. Chalmers CJ, Balmanno K, Hadfield K, Ley R and Cook SJ. Thrombin inhibits Bim (Bcl-2-interacting mediator of cell death) expression and prevents serum-withdrawal-induced apoptosis via protease-activated receptor 1. Biochem J. 2003; 375(Pt 1):99-109.

377. Ritchie H and Fragoyannis A. Thrombin inhibits apoptosis of monocytes and plasminogen activator inhibitor 2 (PAI-2) 
is not responsible for this inhibition. Exp Cell Res. 2000; 260(1):20-29.

378. Pagel CN, de Niese MR, Abraham LA, Chinni C, Song SJ, Pike RN and Mackie EJ. Inhibition of osteoblast apoptosis by thrombin. Bone. 2003; 33(4):733-743.

379. Chinni C, de Niese MR, Tew DJ, Jenkins AL, Bottomley SP and Mackie EJ. Thrombin, a survival factor for cultured myoblasts. J Biol Chem. 1999; 274(14):9169-9174.

380. Vaughan PJ, Pike CJ, Cotman CW and Cunningham DD. Thrombin receptor activation protects neurons and astrocytes from cell death produced by environmental insults. J Neurosci. 1995; 15(7 Pt 2):5389-5401.

381. Turgeon VL, Lloyd ED, Wang S, Festoff BW and Houenou LJ. Thrombin perturbs neurite outgrowth and induces apoptotic cell death in enriched chick spinal motoneuron cultures through caspase activation. J Neurosci. 1998; 18(17):6882-6891.

382. Smirnova IV, Zhang SX, Citron BA, Arnold PM and Festoff BW. Thrombin is an extracellular signal that activates intracellular death protease pathways inducing apoptosis in model motor neurons. J Neurobiol. 1998; 36(1):64-80.

383. Rao HV, Thirumangalakudi L, Desmond P and Grammas P. Cyclin D1, cdk4, and Bim are involved in thrombin-induced apoptosis in cultured cortical neurons. J Neurochem. 2007; 101(2):498-505.

384. Sanphui P, Pramanik SK, Chatterjee N, Moorthi P, Banerji $\mathrm{B}$ and Biswas SC. Efficacy of cyclin dependent kinase 4 inhibitors as potent neuroprotective agents against insults relevant to Alzheimer's disease. PLoS One. 2013; 8(11): e78842.

385. Sionov RV and Haupt Y. The cellular response to p53: the decision between life and death. Oncogene. 1999; 18(45):6145-6157.

386. Borensztajn KS, Bijlsma MF, Groot AP, Bruggemann LW, Versteeg HH, Reitsma PH, Peppelenbosch MP and Spek CA. Coagulation factor Xa drives tumor cells into apoptosis through BH3-only protein Bim up-regulation. Exp Cell Res. 2007; 313(12):2622-2633.

387. Thevenet J, Angelillo-Scherrer A, Price M and Hirt L. Coagulation factor Xa activates thrombin in ischemic neural tissue. J Neurochem. 2009; 111(3):828-836.

388. Sanphui $\mathrm{P}$ and Biswas SC. FoxO3a is activated and executes neuron death via Bim in response to beta-amyloid. Cell Death Dis. 2013; 4:e625.

389. Yin KJ, Lee JM, Chen SD, Xu J and Hsu CY. Amyloid-beta induces Smac release via AP-1/Bim activation in cerebral endothelial cells. J Neurosci. 2002; 22(22):9764-9770.

390. Yao M, Nguyen TV and Pike CJ. Estrogen regulates Bcl-w and Bim expression: role in protection against betaamyloid peptide-induced neuronal death. J Neurosci. 2007; 27(6):1422-1433.

391. Yin KJ, Hsu CY, Hu XY, Chen H, Chen SW, Xu J and Lee JM. Protein phosphatase 2A regulates bim expression via the Akt/FKHRL1 signaling pathway in amyloid-beta peptide-induced cerebrovascular endothelial cell death. J Neurosci. 2006; 26(8):2290-2299.

392. Fischer SF, Bouillet P, O’Donnell K, Light A, Tarlinton DM and Strasser A. Proapoptotic BH3-only protein Bim is essential for developmentally programmed death of germinal center-derived memory B cells and antibodyforming cells. Blood. 2007; 110(12):3978-3984.

393. Wojciechowski S, Tripathi P, Bourdeau T, Acero L, Grimes HL, Katz JD, Finkelman FD and Hildeman DA. Bim/Bcl-2 balance is critical for maintaining naive and memory $\mathrm{T}$ cell homeostasis. J Exp Med. 2007; 204(7):1665-1675.

394. Zhan Y, Zhang Y, Gray D, Carrington EM, Bouillet P, Ko HJ, O’Reilly L, Wicks IP, Strasser A and Lew AM. Defects in the Bcl-2-regulated apoptotic pathway lead to preferential increase of CD25 low Foxp3+ anergic CD4+ T cells. J Immunol. 2011; 187(4):1566-1577.

395. Pellegrini M, Belz G, Bouillet $P$ and Strasser A. Shutdown of an acute $\mathrm{T}$ cell immune response to viral infection is mediated by the proapoptotic Bcl-2 homology 3-only protein Bim. Proc Natl Acad Sci U S A. 2003; 100(24):14175-14180.

396. Hutcheson J, Scatizzi JC, Bickel E, Brown NJ, Bouillet P, Strasser A and Perlman H. Combined loss of proapoptotic genes Bak or Bax with Bim synergizes to cause defects in hematopoiesis and in thymocyte apoptosis. J Exp Med. 2005; 201(12):1949-1960.

397. Hutcheson J, Scatizzi JC, Siddiqui AM, Haines GK, 3rd, Wu T, Li QZ, Davis LS, Mohan C and Perlman H. Combined deficiency of proapoptotic regulators Bim and Fas results in the early onset of systemic autoimmunity. Immunity. 2008; 28(2):206-217.

398. Hughes PD, Belz GT, Fortner KA, Budd RC, Strasser A and Bouillet P. Apoptosis regulators Fas and Bim cooperate in shutdown of chronic immune responses and prevention of autoimmunity. Immunity. 2008; 28(2):197-205.

399. Weant AE, Michalek RD, Khan IU, Holbrook BC, Willingham MC and Grayson JM. Apoptosis regulators Bim and Fas function concurrently to control autoimmunity and CD8+ T cell contraction. Immunity. 2008; 28(2):218230 .

400. Labi V, Woess C, Tuzlak S, Erlacher M, Bouillet P, Strasser A, Tzankov A and Villunger A. Deregulated cell death and lymphocyte homeostasis cause premature lethality in mice lacking the BH3-only proteins Bim and Bmf. Blood. 2014; 123(17):2652-2662.

401. Hu Q, Sader A, Parkman JC and Baldwin TA. Bimmediated apoptosis is not necessary for thymic negative selection to ubiquitous self-antigens. J Immunol. 2009; 183(12):7761-7767.

402. Bunin A, Khwaja FW and Kersh GJ. Regulation of Bim by TCR signals in CD4/CD8 double-positive thymocytes. J Immunol. 2005; 175(3):1532-1539.

403. Mandal M, Crusio KM, Meng F, Liu S, Kinsella M, Clark MR, Takeuchi O and Aifantis I. Regulation of lymphocyte 
progenitor survival by the proapoptotic activities of Bim and Bid. Proc Natl Acad Sci U S A. 2008; 105(52):2084020845.

404. Villunger A, Marsden VS, Zhan Y, Erlacher M, Lew AM, Bouillet P, Berzins S, Godfrey DI, Heath WR and Strasser A. Negative selection of semimature CD4(+)8(-) $\mathrm{HSA}+$ thymocytes requires the $\mathrm{BH} 3$-only protein Bim but is independent of death receptor signaling. Proc Natl Acad Sci U S A. 2004; 101(18):7052-7057.

405. Hu QN and Baldwin TA. Differential roles for bim and nur77 in thymocyte clonal deletion induced by ubiquitous self-antigen. J Immunol. 2015; 194(6):2643-2653.

406. Suen AY and Baldwin TA. Proapoptotic protein Bim is differentially required during thymic clonal deletion to ubiquitous versus tissue-restricted antigens. Proc Natl Acad Sci U S A. 2012; 109(3):893-898.

407. Hale JS, Nelson LT, Simmons KB and Fink PJ. Bcl-2interacting mediator of cell death influences autoantigendriven deletion and TCR revision. J Immunol. 2011; 186(2):799-806.

408. Jay DC, Mitchell DM and Williams MA. Bim mediates the elimination of functionally unfit Th1 responders from the memory pool. PLoS One. 2013; 8(6):e67363.

409. Gibbons RM, Liu X, Pulko V, Harrington SM, Krco CJ, Kwon ED and Dong H. B7-H1 limits the entry of effector CD8(+) T cells to the memory pool by upregulating Bim. Oncoimmunology. 2012; 1(7):1061-1073.

410. Webster RM. The immune checkpoint inhibitors: where are we now? Nat Rev Drug Discov. 2014; 13(12):883-884.

411. Kurtulus S, Sholl A, Toe J, Tripathi P, Raynor J, Li KP, Pellegrini $M$ and Hildeman DA. Bim controls IL-15 availability and limits engagement of multiple BH3-only proteins. Cell Death Differ. 2015; 22(1):174-184.

412. Tsukamoto H, Huston GE, Dibble J, Duso DK and Swain SL. Bim dictates naive CD4 T cell lifespan and the development of age-associated functional defects. J Immunol. 2010; 185(8):4535-4544.

413. Vukmanovic-Stejic M, Zhang Y, Cook JE, Fletcher JM, McQuaid A, Masters JE, Rustin MH, Taams LS, Beverley PC, Macallan DC and Akbar AN. Human CD4+ CD25hi Foxp3+ regulatory $\mathrm{T}$ cells are derived by rapid turnover of memory populations in vivo. J Clin Invest. 2006; 116(9):2423-2433.

414. Tischner D, Gaggl I, Peschel I, Kaufmann M, Tuzlak S, Drach M, Thuille N, Villunger A and Jan Wiegers G. Defective cell death signalling along the Bcl-2 regulated apoptosis pathway compromises Treg cell development and limits their functionality in mice. J Autoimmun. 2012; 38(1):59-69.

415. Reckling S, Divanovic S, Karp CL, Wojciechowski S, Belkaid Y and Hildeman D. Proapoptotic Bcl-2 family member Bim promotes persistent infection and limits protective immunity. Infect Immun. 2008; 76(3):11791185 .
416. Szegezdi E, Kiss I, Simon A, Blasko B, Reichert U, Michel S, Sandor M, Fesus L and Szondy Z. Ligation of retinoic acid receptor alpha regulates negative selection of thymocytes by inhibiting both DNA binding of nur77 and synthesis of bim. J Immunol. 2003; 170(7):3577-3584.

417. Lin B, Kolluri SK, Lin F, Liu W, Han YH, Cao X, Dawson MI, Reed JC and Zhang XK. Conversion of Bcl-2 from protector to killer by interaction with nuclear orphan receptor Nur77/TR3. Cell. 2004; 116(4):527-540.

418. Kiss B, Toth K, Sarang Z, Garabuczi E and Szondy Z. Retinoids induce Nur77-dependent apoptosis in mouse thymocytes. Biochim Biophys Acta. 2015; 1854(3):660670 .

419. Larrubia JR, Benito-Martinez S, Miquel J, Calvino M, Sanz-de-Villalobos E, Gonzalez-Praetorius A, Albertos S, Garcia-Garzon S, Lokhande M and Parra-Cid T. Bimmediated apoptosis and PD-1/PD-L1 pathway impair reactivity of $\mathrm{PD} 1(+) / \mathrm{CD} 127(-) \mathrm{HCV}$-specific $\mathrm{CD} 8(+)$ cells targeting the virus in chronic hepatitis $\mathrm{C}$ virus infection. Cell Immunol. 2011; 269(2):104-114.

420. Wang C, Wen T, Routy JP, Bernard NF, Sekaly RP and Watts TH. 4-1BBL induces TNF receptor-associated factor 1-dependent Bim modulation in human $\mathrm{T}$ cells and is a critical component in the costimulation-dependent rescue of functionally impaired HIV-specific CD8 T cells. J Immunol. 2007; 179(12):8252-8263.

421. Saoulli K, Lee SY, Cannons JL, Yeh WC, Santana A, Goldstein MD, Bangia N, DeBenedette MA, Mak TW, Choi $\mathrm{Y}$ and Watts TH. CD28-independent, TRAF2-dependent costimulation of resting T cells by 4-1BB ligand. J Exp Med. 1998; 187(11):1849-1862.

422. Sabbagh L, Srokowski CC, Pulle G, Snell LM, Sedgmen BJ, Liu Y, Tsitsikov EN and Watts TH. A critical role for TNF receptor-associated factor 1 and Bim down-regulation in CD8 memory T cell survival. Proc Natl Acad Sci U S A. 2006; 103(49):18703-18708.

423. Li SY and Liu Y. Immunotherapy of melanoma with the immune costimulatory monoclonal antibodies targeting CD137. Clin Pharmacol. 2013; 5(Suppl 1):47-53.

424. Craxton A, Draves KE, Gruppi A and Clark EA. BAFF regulates B cell survival by downregulating the BH3-only family member Bim via the ERK pathway. J Exp Med. 2005; 202(10):1363-1374.

425. Lesley R, Xu Y, Kalled SL, Hess DM, Schwab SR, Shu HB and Cyster JG. Reduced competitiveness of autoantigenengaged $\mathrm{B}$ cells due to increased dependence on BAFF. Immunity. 2004; 20(4):441-453.

426. Oliver PM, Vass T, Kappler J and Marrack P. Loss of the proapoptotic protein, Bim, breaks B cell anergy. J Exp Med. 2006; 203(3):731-741.

427. Goodyear CS, Corr M, Sugiyama F, Boyle DL and Silverman GJ. Cutting Edge: Bim is required for superantigen-mediated B cell death. J Immunol. 2007; 178(5):2636-2640. 
428. Craxton A, Draves KE and Clark EA. Bim regulates BCRinduced entry of B cells into the cell cycle. Eur J Immunol. 2007; 37(10):2715-2722.

429. Macauley MS and Paulson JC. Siglecs induce tolerance to cell surface antigens by BIM-dependent deletion of the antigen-reactive B cells. J Immunol. 2014; 193(9):43124321.

430. Kirschnek S, Ying S, Fischer SF, Hacker H, Villunger A, Hochrein $\mathrm{H}$ and Hacker G. Phagocytosis-induced apoptosis in macrophages is mediated by up-regulation and activation of the Bcl-2 homology domain 3-only protein Bim. J Immunol. 2005; 174(2):671-679.

431. Hacker G, Suttner K, Harada H and Kirschnek S. TLRdependent Bim phosphorylation in macrophages is mediated by ERK and is connected to proteasomal degradation of the protein. Int Immunol. 2006; 18(12):1749-1757.

432. Chen M, Huang L and Wang J. Deficiency of Bim in dendritic cells contributes to overactivation of lymphocytes and autoimmunity. Blood. 2007; 109(10):4360-4367.

433. Andina N, Conus S, Schneider EM, Fey MF and Simon HU. Induction of Bim limits cytokine-mediated prolonged survival of neutrophils. Cell Death Differ. 2009; 16(9):1248-1255.

434. Cowburn AS, Summers C, Dunmore BJ, Farahi N, Hayhoe RP, Print CG, Cook SJ and Chilvers ER. Granulocyte/ macrophage colony-stimulating factor causes a paradoxical increase in the $\mathrm{BH} 3$-only pro-apoptotic protein Bim in human neutrophils. Am J Respir Cell Mol Biol. 2011; 44(6):879-887.

435. Bauer A, Kirschnek S and Hacker G. Inhibition of apoptosis can be accompanied by increased Bim levels in T lymphocytes and neutrophil granulocytes. Cell Death Differ. 2007; 14(9):1714-1716.

436. Min-Oo G, Bezman NA, Madera S, Sun JC and Lanier LL. Proapoptotic Bim regulates antigen-specific NK cell contraction and the generation of the memory NK cell pool after cytomegalovirus infection. J Exp Med. 2014; 211(7):1289-1296.

437. Sharma N, Kumar V, Everingham S, Mali RS, Kapur R, Zeng LF, Zhang ZY, Feng GS, Hartmann K, Roers A and Craig AW. SH2 domain-containing phosphatase 2 is a critical regulator of connective tissue mast cell survival and homeostasis in mice. Mol Cell Biol. 2012; 32(14):26532663.

438. Kovarova M and Koller BH. PGE(2) promotes apoptosis induced by cytokine deprivation through EP3 receptor and induces Bim in mouse mast cells. PLoS One. 2014; 9(7):e102948

439. Liu B, Meng D, Wei T, Zhang S, Hu Y and Wang M. Apoptosis and pro-inflammatory cytokine response of mast cells induced by influenza A viruses. PLoS One. 2014; 9(6):e100109.

440. Kozuma Y, Ninomiya H, Murata S, Kono T, Mukai HY and Kojima $\mathrm{H}$. The pro-apoptotic $\mathrm{BH} 3$-only protein
Bim regulates cell cycle progression of hematopoietic progenitors during megakaryopoiesis. J Thromb Haemost. 2010; 8(5):1088-1097.

441. Bunk EC, Konig HG, Bernas T, Engel T, Henshall DC, Kirby BP and Prehn JH. BH3-only proteins BIM and PUMA in the regulation of survival and neuronal differentiation of newly generated cells in the adult mouse hippocampus. Cell Death Dis. 2010; 1:e15.

442. Liang Y, Mirnics ZK, Yan C, Nylander KD and Schor NF. Bcl-2 mediates induction of neural differentiation. Oncogene. 2003; 22(35):5515-5518.

443. Chen DF, Schneider GE, Martinou JC and Tonegawa $\mathrm{S}$. Bcl-2 promotes regeneration of severed axons in mammalian CNS. Nature. 1997; 385(6615):434-439.

444. Zhou J, Li H, Li X, Zhang G, Niu Y, Yuan Z, Herrup K, Zhang YW, Bu G, Xu H and Zhang J. The roles of Cdk5mediated subcellular localization of FOXO1 in neuronal death. J Neurosci. 2015; 35(6):2624-2635.

445. Perier C, Bove J, Wu DC, Dehay B, Choi DK, JacksonLewis V, Rathke-Hartlieb S, Bouillet P, Strasser A, Schulz JB, Przedborski S and Vila M. Two molecular pathways initiate mitochondria-dependent dopaminergic neurodegeneration in experimental Parkinson's disease. Proc Natl Acad Sci U S A. 2007; 104(19):8161-8166.

446. Hunot S, Vila M, Teismann P, Davis RJ, Hirsch EC, Przedborski S, Rakic $\mathrm{P}$ and Flavell RA. JNKmediated induction of cyclooxygenase 2 is required for neurodegeneration in a mouse model of Parkinson's disease. Proc Natl Acad Sci U S A. 2004; 101(2):665-670.

447. Arumugam TV, Cheng YL, Choi Y, Choi YH, Yang S, Yun YK, Park JS, Yang DK, Thundyil J, Gelderblom M, Karamyan VT, Tang SC, Chan SL, Magnus T, Sobey CG and Jo DG. Evidence that gamma-secretase-mediated Notch signaling induces neuronal cell death via the nuclear factorkappaB-Bcl-2-interacting mediator of cell death pathway in ischemic stroke. Mol Pharmacol. 2011; 80(1):23-31.

448. Wang Z, Azmi AS, Ahmad A, Banerjee S, Wang S, Sarkar FH and Mohammad RM. TW-37, a small-molecule inhibitor of $\mathrm{Bcl}-2$, inhibits cell growth and induces apoptosis in pancreatic cancer: involvement of Notch-1 signaling pathway. Cancer Res. 2009; 69(7):2757-2765.

449. Colli ML, Nogueira TC, Allagnat F, Cunha DA, Gurzov EN, Cardozo AK, Roivainen M, Op de Beeck A and Eizirik DL. Exposure to the viral by-product dsRNA or Coxsackievirus B5 triggers pancreatic beta cell apoptosis via a Bim / Mcl-1 imbalance. PLoS Pathog. 2011; 7(9):e1002267.

450. Wali JA, Rondas D, McKenzie MD, Zhao Y, Elkerbout L, Fynch S, Gurzov EN, Akira S, Mathieu C, Kay TW, Overbergh L, Strasser A and Thomas HE. The proapoptotic BH3-only proteins Bim and Puma are downstream of endoplasmic reticulum and mitochondrial oxidative stress in pancreatic islets in response to glucotoxicity. Cell Death Dis. 2014; 5:e1124.

451. McKenzie MD, Jamieson E, Jansen ES, Scott CL, Huang 
DC, Bouillet P, Allison J, Kay TW, Strasser A and Thomas HE. Glucose induces pancreatic islet cell apoptosis that requires the $\mathrm{BH} 3$-only proteins Bim and Puma and multiBH domain protein Bax. Diabetes. 2010; 59(3):644-652.

452. Liston A, Lesage S, Gray DH, O'Reilly LA, Strasser A, Fahrer AM, Boyd RL, Wilson J, Baxter AG, Gallo EM, Crabtree GR, Peng K, Wilson SR and Goodnow CC. Generalized resistance to thymic deletion in the NOD mouse; a polygenic trait characterized by defective induction of Bim. Immunity. 2004; 21(6):817-830.

453. Chen GY and Nunez G. Sterile inflammation: sensing and reacting to damage. Nat Rev Immunol. 2010; 10(12):826837.

454. Marroqui L, Santin I, Dos Santos RS, Marselli L, Marchetti $\mathrm{P}$ and Eizirik DL. BACH2, a candidate risk gene for type 1 diabetes, regulates apoptosis in pancreatic beta-cells via JNK1 modulation and crosstalk with the candidate gene PTPN2. Diabetes. 2014; 63(7):2516-2527.

455. Floyel T, Brorsson C, Nielsen LB, Miani M, BangBerthelsen $\mathrm{CH}$, Friedrichsen M, Overgaard AJ, Berchtold LA, Wiberg A, Poulsen P, Hansen L, Rosinger S, Boehm BO, Ram R, Nguyen Q, Mehta M, et al. CTSH regulates beta-cell function and disease progression in newly diagnosed type 1 diabetes patients. Proc Natl Acad Sci U S A. 2014; 111(28):10305-10310.

456. Ren D, Sun J, Wang C, Ye H, Mao L, Cheng EH, Bell GI and Polonsky KS. Role of BH3-only molecules Bim and Puma in beta-cell death in Pdx1 deficiency. Diabetes. 2014; 63(8):2744-2750.

457. Ren D, Sun J, Mao L, Ye H and Polonsky KS. BH3-only molecule Bim mediates beta-cell death in IRS2 deficiency. Diabetes. 2014; 63(10):3378-3387.

458. Kaiser G, Gerst F, Michael D, Berchtold S, Friedrich B, Strutz-Seebohm N, Lang F, Haring HU and Ullrich S. Regulation of forkhead box O1 (FOXO1) by protein kinase $\mathrm{B}$ and glucocorticoids: different mechanisms of induction of beta cell death in vitro. Diabetologia. 2013; 56(7):15871595.

459. Ardestani A, Paroni F, Azizi Z, Kaur S, Khobragade V, Yuan T, Frogne T, Tao W, Oberholzer J, Pattou F, Kerr Conte $\mathrm{J}$ and Maedler K. MST1 is a key regulator of beta cell apoptosis and dysfunction in diabetes. Nat Med. 2014; 20(4):385-397.

460. Ye Z, Chen Y, Zhang R, Dai H, Zeng C, Zeng H, Feng H, Du G, Fang H and Cai D. c-Jun N-terminal kinase - c-Jun pathway transactivates Bim to promote osteoarthritis. Can J Physiol Pharmacol. 2014; 92(2):132-139.

461. Scatizzi JC, Hutcheson J, Pope RM, Firestein GS, Koch AE, Mavers M, Smason A, Agrawal H, Haines GK, 3rd, Chandel NS, Hotchkiss RS and Perlman H. Bim-Bcl-2 homology 3 mimetic therapy is effective at suppressing inflammatory arthritis through the activation of myeloid cell apoptosis. Arthritis Rheum. 2010; 62(2):441-451.

462. Kodama T, Hikita H, Kawaguchi T, Saito Y, Tanaka
S, Shigekawa M, Shimizu S, Li W, Miyagi T, Kanto $\mathrm{T}$, Hiramatsu N, Tatsumi $\mathrm{T}$ and Takehara T. The Bcl-2 homology domain 3 (BH3)-only proteins Bim and bid are functionally active and restrained by anti-apoptotic Bcl-2 family proteins in healthy liver. J Biol Chem. 2013; 288(42):30009-30018.

463. Tao GZ, Lehwald N, Jang KY, Baek J, Xu B, Omary MB and Sylvester KG. Wnt/beta-catenin signaling protects mouse liver against oxidative stress-induced apoptosis through the inhibition of forkhead transcription factor FoxO3. J Biol Chem. 2013; 288(24):17214-17224.

464. Badmann A, Keough A, Kaufmann T, Bouillet P, Brunner T and Corazza N. Role of TRAIL and the pro-apoptotic Bcl-2 homolog Bim in acetaminophen-induced liver damage. Cell Death Dis. 2011; 2:e171.

465. Corazza N, Jakob S, Schaer C, Frese S, Keogh A, Stroka D, Kassahn D, Torgler R, Mueller C, Schneider P and Brunner T. TRAIL receptor-mediated JNK activation and Bim phosphorylation critically regulate Fas-mediated liver damage and lethality. J Clin Invest. 2006; 116(9):24932499.

466. Barreyro FJ, Kobayashi S, Bronk SF, Werneburg NW, Malhi $\mathrm{H}$ and Gores GJ. Transcriptional regulation of Bim by FoxO3A mediates hepatocyte lipoapoptosis. J Biol Chem. 2007; 282(37):27141-27154.

467. Malhi H, Bronk SF, Werneburg NW and Gores GJ. Free fatty acids induce JNK-dependent hepatocyte lipoapoptosis. J Biol Chem. 2006; 281(17):12093-12101.

468. Kaufmann T, Jost PJ, Pellegrini M, Puthalakath H, Gugasyan R, Gerondakis S, Cretney E, Smyth MJ, Silke J, Hakem R, Bouillet P, Mak TW, Dixit VM and Strasser A. Fatal hepatitis mediated by tumor necrosis factor TNFalpha requires caspase- 8 and involves the $\mathrm{BH} 3$-only proteins $\mathrm{Bid}$ and Bim. Immunity. 2009; 30(1):56-66.

469. Cazanave SC, Wang X, Zhou H, Rahmani M, Grant S, Durrant DE, Klaassen CD, Yamamoto M and Sanyal AJ. Degradation of Keap1 activates BH3-only proteins Bim and PUMA during hepatocyte lipoapoptosis. Cell Death Differ. 2014; 21(8):1303-1312.

470. Larrubia JR, Lokhande MU, Garcia-Garzon S, Miquel J, Subira D and Sanz-de-Villalobos E. Role of T cell death in maintaining immune tolerance during persistent viral hepatitis. World J Gastroenterol. 2013; 19(12):1877-1889.

471. Wojciechowski S, Jordan MB, Zhu Y, White J, Zajac AJ and Hildeman DA. Bim mediates apoptosis of CD127(lo) effector T cells and limits T cell memory. Eur J Immunol. 2006; 36(7):1694-1706.

472. Larrubia JR, Lokhande MU, Garcia-Garzon S, Miquel J, Gonzalez-Praetorius A, Parra-Cid T and Sanz-de-Villalobos E. Persistent hepatitis $\mathrm{C}$ virus (HCV) infection impairs $\mathrm{HCV}$-specific cytotoxic $\mathrm{T}$ cell reactivity through $\mathrm{Mcl}-1 / \mathrm{Bim}$ imbalance due to CD127 down-regulation. J Viral Hepat. 2013; 20(2):85-94.

473. Holz LE, Benseler V, Bowen DG, Bouillet P, Strasser A, 
O'Reilly L, d'Avigdor WM, Bishop AG, McCaughan GW and Bertolino P. Intrahepatic murine CD8 T-cell activation associates with a distinct phenotype leading to Bimdependent death. Gastroenterology. 2008; 135(3):989-997.

474. Lopes AR, Kellam P, Das A, Dunn C, Kwan A, Turner J, Peppa D, Gilson RJ, Gehring A, Bertoletti A and Maini MK. Bim-mediated deletion of antigen-specific CD8 T cells in patients unable to control HBV infection. J Clin Invest. 2008; 118(5):1835-1845.

475. Grayson JM, Weant AE, Holbrook BC and Hildeman D. Role of Bim in regulating CD8+ T-cell responses during chronic viral infection. J Virol. 2006; 80(17):8627-8638.

476. Sabbagh L, Pulle G, Liu Y, Tsitsikov EN and Watts TH. ERK-dependent Bim modulation downstream of the 4-1BBTRAF1 signaling axis is a critical mediator of CD8 T cell survival in vivo. J Immunol. 2008; 180(12):8093-8101.

477. Wang C, McPherson AJ, Jones RB, Kawamura KS, Lin GH, Lang PA, Ambagala T, Pellegrini M, Calzascia T, Aidarus N, Elford AR, Yue FY, Kremmer E, Kovacs CM, Benko E, Tremblay C, et al. Loss of the signaling adaptor TRAF1 causes CD8+ T cell dysregulation during human and murine chronic infection. J Exp Med. 2012; 209(1):7791.

478. Schuler F, Baumgartner F, Klepsch V, Chamson M, MullerHolzner E, Watson CJ, Oh S, Hennighausen L, Tymoszuk $\mathrm{P}$, Doppler W and Villunger A. The BH3-only protein BIM contributes to late-stage involution in the mouse mammary gland. Cell Death Differ. 2015 Jun 5. doi: 10.1038/ cdd.2015.61. [Epub ahead of print].

479. Sugrue T, Brown JA, Lowndes NF and Ceredig R. Multiple facets of the DNA damage response contribute to the radioresistance of mouse mesenchymal stromal cell lines. Stem Cells. 2013; 31(1):137-145.

480. Egle A, Harris AW, Bouillet P and Cory S. Bim is a suppressor of Myc-induced mouse B cell leukemia. Proc Natl Acad Sci U S A. 2004; 101(16):6164-6169.

481. Vandenberg CJ, Waring P, Strasser A and Cory S. Plasmacytomagenesis in Emu-v-abl transgenic mice is accelerated when apoptosis is restrained. Blood. 2014; 124(7):1099-1109.

482. Tan TT, Degenhardt K, Nelson DA, Beaudoin B, NievesNeira W, Bouillet P, Villunger A, Adams JM and White E. Key roles of BIM-driven apoptosis in epithelial tumors and rational chemotherapy. Cancer Cell. 2005; 7(3):227-238.

483. Tagawa H, Karnan S, Suzuki R, Matsuo K, Zhang X, Ota A, Morishima Y, Nakamura S and Seto M. Genome-wide array-based CGH for mantle cell lymphoma: identification of homozygous deletions of the proapoptotic gene BIM. Oncogene. 2005; 24(8):1348-1358.

484. Greenhough A, Wallam CA, Hicks DJ, Moorghen M, Williams AC and Paraskeva C. The proapoptotic BH3only protein Bim is downregulated in a subset of colorectal cancers and is repressed by antiapoptotic COX-2/PGE(2) signalling in colorectal adenoma cells. Oncogene. 2010;
29(23):3398-3410.

485. Nikrad M, Johnson T, Puthalalath H, Coultas L, Adams $\mathrm{J}$ and Kraft AS. The proteasome inhibitor bortezomib sensitizes cells to killing by death receptor ligand TRAIL via BH3-only proteins Bik and Bim. Mol Cancer Ther. 2005; 4(3):443-449.

486. Dai DL, Wang Y, Liu M, Martinka M and Li G. Bim expression is reduced in human cutaneous melanomas. $\mathrm{J}$ Invest Dermatol. 2008; 128(2):403-407.

487. Cartlidge RA, Thomas GR, Cagnol S, Jong KA, Molton SA, Finch AJ and McMahon M. Oncogenic BRAF(V600E) inhibits BIM expression to promote melanoma cell survival. Pigment Cell Melanoma Res. 2008; 21(5):534-544.

488. Woods NT, Yamaguchi H, Lee FY, Bhalla KN and Wang HG. Anoikis, initiated by Mcl-1 degradation and Bim induction, is deregulated during oncogenesis. Cancer Res. 2007; 67(22):10744-10752.

489. Reginato MJ, Mills KR, Paulus JK, Lynch DK, Sgroi DC, Debnath J, Muthuswamy SK and Brugge JS. Integrins and EGFR coordinately regulate the pro-apoptotic protein Bim to prevent anoikis. Nat Cell Biol. 2003; 5(8):733-740.

490. Uehara N, Matsuoka Y and Tsubura A. Mesothelin promotes anchorage-independent growth and prevents anoikis via extracellular signal-regulated kinase signaling pathway in human breast cancer cells. Mol Cancer Res. 2008; 6(2):186-193.

491. Giannoni E, Buricchi F, Grimaldi G, Parri M, Cialdai F, Taddei ML, Raugei G, Ramponi G and Chiarugi P. Redox regulation of anoikis: reactive oxygen species as essential mediators of cell survival. Cell Death Differ. 2008; 15(5):867-878.

492. Liu K, Liu F, Zhang N, Liu S and Jiang Y. Pokemon Silencing Leads to Bim-Mediated Anoikis of Human Hepatoma Cell QGY7703. Int J Mol Sci. 2012; 13(5):58185831.

493. Collins NL, Reginato MJ, Paulus JK, Sgroi DC, Labaer $\mathrm{J}$ and Brugge JS. G1/S cell cycle arrest provides anoikis resistance through Erk-mediated Bim suppression. Mol Cell Biol. 2005; 25(12):5282-5291.

494. Drury LJ, Wendt MK and Dwinell MB. CXCL12 chemokine expression and secretion regulates colorectal carcinoma cell anoikis through Bim-mediated intrinsic apoptosis. PLoS One. 2010; 5(9):e12895.

495. Whelan KA, Caldwell SA, Shahriari KS, Jackson SR, Franchetti LD, Johannes GJ and Reginato MJ. Hypoxia suppression of Bim and Bmf blocks anoikis and luminal clearing during mammary morphogenesis. Mol Biol Cell. 2010; 21(22):3829-3837.

496. Buchheit CL, Angarola BL, Steiner A, Weigel KJ and Schafer ZT. Anoikis evasion in inflammatory breast cancer cells is mediated by Bim-EL sequestration. Cell Death Differ. 2015; 22(8):1275-1286.

497. Yang JM, O’Neill P, Jin W, Foty R, Medina DJ, Xu Z, Lomas M, Arndt GM, Tang Y, Nakada M, Yan L and 
Hait WN. Extracellular matrix metalloproteinase inducer (CD147) confers resistance of breast cancer cells to Anoikis through inhibition of Bim. J Biol Chem. 2006; 281(14):9719-9727.

498. Generali D, Berruti A, Brizzi MP, Campo L, Bonardi S, Wigfield S, Bersiga A, Allevi G, Milani M, Aguggini S, Gandolfi V, Dogliotti L, Bottini A, Harris AL and Fox SB. Hypoxia-inducible factor-1alpha expression predicts a poor response to primary chemoendocrine therapy and diseasefree survival in primary human breast cancer. Clin Cancer Res. 2006; 12(15):4562-4568.

499. Karakashev SV and Reginato MJ. Hypoxia/HIF1alpha induces lapatinib resistance in ERBB2-positive breast cancer cells via regulation of DUSP2. Oncotarget. 2015; 6(4):1967-1980.

500. Park SH, Ito K, Olcott W, Katsyv I, Halstead-Nussloch $\mathrm{G}$ and Irie HY. PTK6 inhibition promotes apoptosis of Lapatinib-resistant Her2 breast cancer cells by inducing Bim. Breast Cancer Res. 2015; 17(1):86.

501. Belloc F, Moreau-Gaudry F, Uhalde M, Cazalis L, Jeanneteau M, Lacombe F, Praloran V and Mahon FX. Imatinib and nilotinib induce apoptosis of chronic myeloid leukemia cells through a Bim-dependant pathway modulated by cytokines. Cancer Biol Ther. 2007; 6(6):912919.

502. Romagnoli M, Seveno C, Wuilleme-Toumi S, Amiot M, Bataille R, Minvielle S and Barille-Nion S. The imbalance between Survivin and Bim mediates tumour growth and correlates with poor survival in patients with multiple myeloma. Br J Haematol. 2009; 145(2):180-189.

503. Piazza R, Magistroni V, Mogavero A, Andreoni F, Ambrogio C, Chiarle R, Mologni L, Bachmann PS, Lock RB, Collini P, Pelosi G and Gambacorti-Passerini C. Epigenetic silencing of the proapoptotic gene BIM in anaplastic large cell lymphoma through an MeCP2/SIN3a deacetylating complex. Neoplasia. 2013; 15(5):511-522.

504. Gutierrez A, Sanda T, Grebliunaite R, Carracedo A, Salmena L, Ahn Y, Dahlberg S, Neuberg D, Moreau LA, Winter SS, Larson R, Zhang J, Protopopov A, Chin L, Pandolfi PP, Silverman LB, et al. High frequency of PTEN, PI3K, and AKT abnormalities in T-cell acute lymphoblastic leukemia. Blood. 2009; 114(3):647-650.

505. Palomero T, Lim WK, Odom DT, Sulis ML, Real PJ, Margolin A, Barnes KC, O’Neil J, Neuberg D, Weng AP, Aster JC, Sigaux F, Soulier J, Look AT, Young RA, Califano A, et al. NOTCH1 directly regulates c-MYC and activates a feed-forward-loop transcriptional network promoting leukemic cell growth. Proc Natl Acad Sci U S A. 2006; 103(48):18261-18266.

506. Reynolds C, Roderick JE, LaBelle JL, Bird G, Mathieu R, Bodaar K, Colon D, Pyati U, Stevenson KE, Qi J, Harris M, Silverman LB, Sallan SE, Bradner JE, Neuberg DS, Look AT, et al. Repression of BIM mediates survival signaling by MYC and AKT in high-risk T-cell acute lymphoblastic leukemia. Leukemia. 2014; 28(9):1819-1827.
507. Chen S, Zhang Y, Zhou L, Leng Y, Lin H, Kmieciak M, Pei XY, Jones R, Orlowski RZ, Dai Y and Grant S. A Bimtargeting strategy overcomes adaptive bortezomib resistance in myeloma through a novel link between autophagy and apoptosis. Blood. 2014; 124(17):2687-2697.

508. Pei XY, Dai Y, Felthousen J, Chen S, Takabatake Y, Zhou L, Youssefian LE, Sanderson MW, Bodie WW, Kramer LB, Orlowski RZ and Grant S. Circumvention of Mcl1-dependent drug resistance by simultaneous Chk1 and MEK1/2 inhibition in human multiple myeloma cells. PLoS One. 2014; 9(3):e89064.

509. Chinen Y, Kuroda J, Shimura Y, Nagoshi H, Kiyota M, Yamamoto-Sugitani M, Mizutani S, Sakamoto N, Ri M, Kawata E, Kobayashi T, Matsumoto Y, Horiike S, Iida S and Taniwaki M. Phosphoinositide protein kinase PDPK1 is a crucial cell signaling mediator in multiple myeloma. Cancer Res. 2014; 74(24):7418-7429.

510. Murray ME, Gavile CM, Nair JR, Koorella C, Carlson LM, Buac D, Utley A, Chesi M, Bergsagel PL, Boise LH and Lee KP. CD28-mediated pro-survival signaling induces chemotherapeutic resistance in multiple myeloma. Blood. 2014; 123(24):3770-3779.

511. Paterson A, Mockridge CI, Adams JE, Krysov S, Potter KN, Duncombe AS, Cook SJ, Stevenson FK and Packham G. Mechanisms and clinical significance of BIM phosphorylation in chronic lymphocytic leukemia. Blood. 2012; 119(7):1726-1736.

512. Longo PG, Laurenti L, Gobessi S, Sica S, Leone G and Efremov DG. The Akt/Mcl-1 pathway plays a prominent role in mediating antiapoptotic signals downstream of the B-cell receptor in chronic lymphocytic leukemia B cells. Blood. 2008; 111(2):846-855.

513. Pepper C, Lin TT, Pratt G, Hewamana S, Brennan P, Hiller L, Hills R, Ward R, Starczynski J, Austen B, Hooper L, Stankovic T and Fegan C. Mcl-1 expression has in vitro and in vivo significance in chronic lymphocytic leukemia and is associated with other poor prognostic markers. Blood. 2008; 112(9):3807-3817.

514. O'Reilly LA, Kruse EA, Puthalakath H, Kelly PN, Kaufmann T, Huang DC and Strasser A. MEK/ERKmediated phosphorylation of Bim is required to ensure survival of $\mathrm{T}$ and $\mathrm{B}$ lymphocytes during mitogenic stimulation. J Immunol. 2009; 183(1):261-269.

515. Del Gaizo Moore V, Brown JR, Certo M, Love TM, Novina $\mathrm{CD}$ and Letai A. Chronic lymphocytic leukemia requires BCL2 to sequester prodeath BIM, explaining sensitivity to BCL2 antagonist ABT-737. J Clin Invest. 2007; 117(1):112121.

516. Del Gaizo Moore V, Schlis KD, Sallan SE, Armstrong SA and Letai A. BCL-2 dependence and ABT-737 sensitivity in acute lymphoblastic leukemia. Blood. 2008; 111(4):23002309.

517. Iglesias-Serret D, de Frias M, Santidrian AF, Coll-Mulet L, Cosialls AM, Barragan M, Domingo A, Gil J and Pons G. Regulation of the proapoptotic $\mathrm{BH} 3$-only protein BIM by 
glucocorticoids, survival signals and proteasome in chronic lymphocytic leukemia cells. Leukemia. 2007; 21(2):281287.

518. Jing D, Bhadri VA, Beck D, Thoms JA, Yakob NA, Wong JW, Knezevic K, Pimanda JE and Lock RB. Opposing regulation of BIM and BCL2 controls glucocorticoidinduced apoptosis of pediatric acute lymphoblastic leukemia cells. Blood. 2015; 125(2):273-283.

519. Lopez-Royuela N, Balsas P, Galan-Malo P, Anel A, Marzo I and Naval J. Bim is the key mediator of glucocorticoidinduced apoptosis and of its potentiation by rapamycin in human myeloma cells. Biochim Biophys Acta. 2010; 1803(2):311-322.

520. Wang Z, Malone MH, He H, McColl KS and Distelhorst $\mathrm{CW}$. Microarray analysis uncovers the induction of the proapoptotic BH3-only protein Bim in multiple models of glucocorticoid-induced apoptosis. J Biol Chem. 2003; 278(26):23861-23867.

521. Jiang N, Koh GS, Lim JY, Kham SK, Ariffin H, Chew FT and Yeoh AE. BIM is a prognostic biomarker for early prednisolone response in pediatric acute lymphoblastic leukemia. Exp Hematol. 2011; 39(3):321-329, 329 e321323.

522. Bachmann PS, Piazza RG, Janes ME, Wong NC, Davies C, Mogavero A, Bhadri VA, Szymanska B, Geninson G, Magistroni V, Cazzaniga G, Biondi A, Miranda-Saavedra D, Gottgens B, Saffery R, Craig JM, et al. Epigenetic silencing of BIM in glucocorticoid poor-responsive pediatric acute lymphoblastic leukemia, and its reversal by histone deacetylase inhibition. Blood. 2010; 116(16):30133022.

523. Espina B, Liang M, Russell RG and Hulley PA. Regulation of bim in glucocorticoid-mediated osteoblast apoptosis. J Cell Physiol. 2008; 215(2):488-496.

524. Lutzner N, Kalbacher H, Krones-Herzig A and Rosl F. FOXO3 is a glucocorticoid receptor target and regulates LKB1 and its own expression based on cellular AMP levels via a positive autoregulatory loop. PLoS One. 2012; 7(7):e42166.

525. Ma J, Xie Y, Shi Y, Qin W, Zhao B and Jin Y. Glucocorticoid-induced apoptosis requires FOXO3A activity. Biochem Biophys Res Commun. 2008; 377(3):894898.

526. Priceman SJ, Kirzner JD, Nary LJ, Morris D, Shankar DB, Sakamoto KM and Medh RD. Calcium-dependent upregulation of E4BP4 expression correlates with glucocorticoid-evoked apoptosis of human leukemic CEM cells. Biochem Biophys Res Commun. 2006; 344(2):491499.

527. Beach JA, Nary LJ, Hirakawa Y, Holland E, Hovanessian $\mathrm{R}$ and Medh RD. E4BP4 facilitates glucocorticoid-evoked apoptosis of human leukemic CEM cells via upregulation of Bim. J Mol Signal. 2011; 6(1):13.

528. Chen F, Zhang L, OuYang Y, Guan H, Liu Q and Ni B.
Glucocorticoid induced osteoblast apoptosis by increasing E4BP4 expression via up-regulation of Bim. Calcif Tissue Int. 2014; 94(6):640-647.

529. Asselin-Labat ML, David M, Biola-Vidamment A, Lecoeuche D, Zennaro MC, Bertoglio J and Pallardy M. GILZ, a new target for the transcription factor FoxO3, protects $\mathrm{T}$ lymphocytes from interleukin-2 withdrawalinduced apoptosis. Blood. 2004; 104(1):215-223.

530. Latre de Late P, Pepin A, Assaf-Vandecasteele H, Espinasse C, Nicolas V, Asselin-Labat ML, Bertoglio J, Pallardy $\mathrm{M}$ and Biola-Vidamment A. Glucocorticoid-induced leucine zipper (GILZ) promotes the nuclear exclusion of FOXO3 in a Crm1-dependent manner. J Biol Chem. 2010; 285(8):5594-5605.

531. Wasim M, Carlet M, Mansha M, Greil R, Ploner C, Trockenbacher A, Rainer J and Kofler R. PLZF/ZBTB16, a glucocorticoid response gene in acute lymphoblastic leukemia, interferes with glucocorticoid-induced apoptosis. J Steroid Biochem Mol Biol. 2010; 120(4-5):218-227.

532. Hosono N, Kishi S, Iho S, Urasaki Y, Yoshida A, Kurooka H, Yokota Y and Ueda T. Glutathione S-transferase M1 inhibits dexamethasone-induced apoptosis in association with the suppression of Bim through dual mechanisms in a lymphoblastic leukemia cell line. Cancer Sci. 2010; 101(3):767-773.

533. Gu L, Zhou C, Liu H, Gao J, Li Q, Mu D and Ma Z. Rapamycin sensitizes T-ALL cells to dexamethasoneinduced apoptosis. J Exp Clin Cancer Res. 2010; 29:150.

534. Wei G, Twomey D, Lamb J, Schlis K, Agarwal J, Stam RW, Opferman JT, Sallan SE, den Boer ML, Pieters R, Golub TR and Armstrong SA. Gene expression-based chemical genomics identifies rapamycin as a modulator of MCL1 and glucocorticoid resistance. Cancer Cell. 2006; 10(4):331-342.

535. Berges C, Haberstock H, Fuchs D, Sadeghi M, Opelz G, Daniel V and Naujokat C. Proteasome inhibition activates the mitochondrial pathway of apoptosis in human CD4+ T cells. J Cell Biochem. 2009; 108(4):935-946.

536. de Queiroz Crusoe E, Maiso P, Fernandez-Lazaro D, SanSegundo L, Garayoa M, Garcia-Gomez A, Gutierrez NC, Delgado M, Colado E, Martin-Sanchez J, Lee FY and Ocio EM. Transcriptomic rationale for the synergy observed with dasatinib + bortezomib + dexamethasone in multiple myeloma. Ann Hematol. 2012; 91(2):257-269.

537. Rapino F, Naumann I and Fulda S. Bortezomib antagonizes microtubule-interfering drug-induced apoptosis by inhibiting G2/M transition and MCL-1 degradation. Cell Death Dis. 2013; 4:e925.

538. Lambrou GI, Papadimitriou L, Chrousos GP and Vlahopoulos SA. Glucocorticoid and proteasome inhibitor impact on the leukemic lymphoblast: multiple, diverse signals converging on a few key downstream regulators. Mol Cell Endocrinol. 2012; 351(2):142-151.

539. Zantl N, Weirich G, Zall H, Seiffert BM, Fischer SF, 
Kirschnek S, Hartmann C, Fritsch RM, Gillissen B, Daniel PT and Hacker G. Frequent loss of expression of the proapoptotic protein Bim in renal cell carcinoma: evidence for contribution to apoptosis resistance. Oncogene. 2007; 26(49):7038-7048.

540. Li J, Zhang Z and Li G. Patient outcome prediction using multiple biomarkers in human melanoma: A clinicopathological study of 118 cases. Exp Ther Med. 2011; 2(1):131-135.

541. Paraiso KH, Xiang Y, Rebecca VW, Abel EV, Chen YA, Munko AC, Wood E, Fedorenko IV, Sondak VK, Anderson AR, Ribas A, Palma MD, Nathanson KL, Koomen JM, Messina JL and Smalley KS. PTEN loss confers BRAF inhibitor resistance to melanoma cells through the suppression of BIM expression. Cancer Res. 2011; 71(7):2750-2760.

542. Cheriyath V, Kuhns MA, Jacobs BS, Evangelista P, Elson P, Downs-Kelly E, Tubbs R and Borden EC. G1P3, an interferon- and estrogen-induced survival protein contributes to hyperplasia, tamoxifen resistance and poor outcomes in breast cancer. Oncogene. 2012; 31(17):22222236.

543. Lv Y, Song S, Zhang K, Gao H and Ma R. CHIP regulates AKT/FoxO/Bim signaling in MCF7 and MCF10A cells. PLoS One. 2013; 8(12):e83312.

544. Ahmed SF, Deb S, Paul I, Chatterjee A, Mandal T, Chatterjee U and Ghosh MK. The chaperone-assisted E3 ligase $\mathrm{C}$ terminus of Hsc70-interacting protein (CHIP) targets PTEN for proteasomal degradation. J Biol Chem. 2012; 287(19):15996-16006.

545. Hu W, Lu SX, Li M, Zhang C, Liu LL, Fu J, Jin JT, Luo RZ, Zhang CZ and Yun JP. Pyruvate kinase M2 prevents apoptosis via modulating Bim stability and associates with poor outcome in hepatocellular carcinoma. Oncotarget. 2015; 6(9):6570-6583.

546. Karachaliou N, Rosell R, Molina MA and Viteri S. Predicting resistance by selection of signaling pathways. Transl Lung Cancer Res. 2014; 3(2):107-115.

547. Rosell R, Carcereny E, Gervais R, Vergnenegre A, Massuti B, Felip E, Palmero R, Garcia-Gomez R, Pallares C, Sanchez JM, Porta R, Cobo M, Garrido P, Longo F, Moran $\mathrm{T}$, Insa A, et al. Erlotinib versus standard chemotherapy as first-line treatment for European patients with advanced EGFR mutation-positive non-small-cell lung cancer (EURTAC): a multicentre, open-label, randomised phase 3 trial. Lancet Oncol. 2012; 13(3):239-246.

548. Costa C, Molina MA, Drozdowskyj A, Gimenez-Capitan A, Bertran-Alamillo J, Karachaliou N, Gervais R, Massuti B, Wei J, Moran T, Majem M, Felip E, Carcereny E, Garcia-Campelo R, Viteri S, Taron M, et al. The impact of EGFR T790M mutations and BIM mRNA expression on outcome in patients with EGFR-mutant NSCLC treated with erlotinib or chemotherapy in the randomized phase III EURTAC trial. Clin Cancer Res. 2014; 20(7):2001-2010.

549. Gordon PM and Fisher DE. Role for the proapoptotic factor
BIM in mediating imatinib-induced apoptosis in a c-KITdependent gastrointestinal stromal tumor cell line. J Biol Chem. 2010; 285(19):14109-14114.

550. Ambrosini G, Seelman SL and Schwartz GK. Differentiation-related gene-1 decreases Bim stability by proteasome-mediated degradation. Cancer Res. 2009; 69(15):6115-6121.

551. Zhang W, Cheng GZ, Gong J, Hermanto U, Zong CS, Chan J, Cheng JQ and Wang LH. RACK1 and CIS mediate the degradation of BimEL in cancer cells. J Biol Chem. 2008; 283(24):16416-16426.

552. Principe DR, Doll JA, Bauer J, Jung B, Munshi HG, Bartholin L, Pasche B, Lee C and Grippo PJ. TGFbeta: duality of function between tumor prevention and carcinogenesis. J Natl Cancer Inst. 2014; 106(2):djt369.

553. Ramjaun AR, Tomlinson S, Eddaoudi A and Downward J. Upregulation of two BH3-only proteins, Bmf and Bim, during TGF beta-induced apoptosis. Oncogene. 2007; 26(7):970-981.

554. Wiener Z, Band AM, Kallio P, Hogstrom J, Hyvonen V, Kaijalainen S, Ritvos O, Haglund C, Kruuna O, Robine S, Louvard D, Ben-Neriah Y and Alitalo K. Oncogenic mutations in intestinal adenomas regulate Bim-mediated apoptosis induced by TGF-beta. Proc Natl Acad Sci U S A. 2014; 111(21):E2229-2236.

555. Houde N, Chamoux E, Bisson M and Roux S. Transforming growth factor-betal (TGF-beta1) induces human osteoclast apoptosis by up-regulating Bim. J Biol Chem. 2009; 284(35):23397-23404.

556. Cash TP, Gruber JJ, Hartman TR, Henske EP and Simon MC. Loss of the Birt-Hogg-Dube tumor suppressor results in apoptotic resistance due to aberrant TGFbeta-mediated transcription. Oncogene. 2011; 30(22):2534-2546.

557. Fernando J, Sancho P, Fernandez-Rodriguez CM, Lledo JL, Caja L, Campbell JS, Fausto N and Fabregat I. Sorafenib sensitizes hepatocellular carcinoma cells to physiological apoptotic stimuli. J Cell Physiol. 2012; 227(4):1319-1325.

558. Gordian E, Li J, Pevzner Y, Mediavilla-Varela M, Luddy K, Ohaegbulam K, Daniel KG, Haura EB and Munoz-Antonia T. Transforming growth factor beta signaling overcomes dasatinib resistance in lung cancer. PLoS One. 2014; 9(12):e114131.

559. Smith AL, Iwanaga R, Drasin DJ, Micalizzi DS, Vartuli RL, Tan AC and Ford HL. The miR-106b-25 cluster targets Smad7, activates TGF-beta signaling, and induces EMT and tumor initiating cell characteristics downstream of Six1 in human breast cancer. Oncogene. 2012; 31(50):5162-5171.

560. Meyer C, Liu Y, Kaul A, Peipe I and Dooley S. Caveolin-1 abrogates TGF-beta mediated hepatocyte apoptosis. Cell Death Dis. 2013; 4:e466.

561. Hoshino Y, Katsuno Y, Ehata S and Miyazono K. Autocrine TGF-beta protects breast cancer cells from apoptosis through reduction of $\mathrm{BH} 3$-only protein, Bim. J Biochem. 2011; 149(1):55-65. 
562. van der Heide LP, van Dinther M, Moustakas A and ten Dijke P. TGFbeta activates mitogen- and stress-activated protein kinase-1 (MSK1) to attenuate cell death. J Biol Chem. 2011; 286(7):5003-5011.

563. Kover K, Yan Y, Tong PY, Watkins D, Li X, Tasch J, Hager M, Clements M and Moore WV. Osteocalcin protects pancreatic beta cell function and survival under high glucose conditions. Biochem Biophys Res Commun. 2015; 462(1):21-26.

564. Qin F, Tian J, Zhou D and Chen L. Mst1 and Mst2 kinases: regulations and diseases. Cell Biosci. 2013; 3(1):31.

565. Chen J, Saxena G, Mungrue IN, Lusis AJ and Shalev A. Thioredoxin-interacting protein: a critical link between glucose toxicity and beta-cell apoptosis. Diabetes. 2008; 57(4):938-944.

566. Reich E, Tamary A, Sionov RV and Melloul D. Involvement of thioredoxin-interacting protein (TXNIP) in glucocorticoid-mediated beta cell death. Diabetologia. 2012; 55(4):1048-1057.

567. Lee YS and Jun HS. Anti-diabetic actions of glucagonlike peptide-1 on pancreatic beta-cells. Metabolism. 2014; 63(1):9-19.

568. Salcedo I, Tweedie D, Li Y and Greig NH. Neuroprotective and neurotrophic actions of glucagon-like peptide-1: an emerging opportunity to treat neurodegenerative and cerebrovascular disorders. Br J Pharmacol. 2012; 166(5):1586-1599.

569. Meredith ME, Salameh TS and Banks WA. Intranasal Delivery of Proteins and Peptides in the Treatment of Neurodegenerative Diseases. AAPS J. 2015; 17(4):780-787.

570. Igoillo-Esteve M, Gurgul-Convey E, Hu A, Romagueira Bichara Dos Santos L, Abdulkarim B, Chintawar S, Marselli L, Marchetti P, Jonas JC, Eizirik DL, Pandolfo M and Cnop M. Unveiling a common mechanism of apoptosis in beta-cells and neurons in Friedreich's ataxia. Hum Mol Genet. 2015; 24(8):2274-2286.

571. Oltersdorf T, Elmore SW, Shoemaker AR, Armstrong RC, Augeri DJ, Belli BA, Bruncko M, Deckwerth TL, Dinges J, Hajduk PJ, Joseph MK, Kitada S, Korsmeyer SJ, Kunzer $\mathrm{AR}$, Letai A, Li C, et al. An inhibitor of Bcl-2 family proteins induces regression of solid tumours. Nature. 2005; 435(7042):677-681.

572. Deng J, Carlson N, Takeyama K, Dal Cin P, Shipp M and Letai A. BH3 profiling identifies three distinct classes of apoptotic blocks to predict response to ABT-737 and conventional chemotherapeutic agents. Cancer Cell. 2007; 12(2):171-185.

573. Chen S, Dai Y, Pei XY and Grant S. Bim upregulation by histone deacetylase inhibitors mediates interactions with the Bcl-2 antagonist ABT-737: evidence for distinct roles for Bcl-2, Bcl-xL, and Mcl-1. Mol Cell Biol. 2009; 29(23):6149-6169.

574. Cetin Z, Ozbilim G, Erdogan A, Luleci G and Karauzum $\mathrm{SB}$. Evaluation of PTEN and Mcl-1 expressions in NSCLC expressing wild-type or mutated EGFR. Med Oncol. 2010; 27(3):853-860.

575. Jebahi A, Villedieu M, Petigny-Lechartier C, Brotin E, Louis MH, Abeilard E, Giffard F, Guercio M, Briand M, Gauduchon P, Lheureux S and Poulain L. PI3K/mTOR dual inhibitor NVP-BEZ235 decreases Mcl-1 expression and sensitizes ovarian carcinoma cells to Bcl-xL-targeting strategies, provided that Bim expression is induced. Cancer Lett. 2014; 348(1-2):38-49.

576. Rahmani M, Aust MM, Attkisson E, Williams DC, Jr., Ferreira-Gonzalez A and Grant S. Dual inhibition of Bcl2 and Bcl-xL strikingly enhances PI3K inhibition-induced apoptosis in human myeloid leukemia cells through a GSK3- and Bim-dependent mechanism. Cancer Res. 2013; 73(4):1340-1351.

577. Lheureux S, N'Diaye M, Blanc-Fournier C, Dugue AE, Clarisse B, Dutoit S, Giffard F, Abeilard E, Briand M, Labiche A, Grellard JM, Crouet H, Martin S, Joly F and Poulain L. Identification of predictive factors of response to the BH3-mimetic molecule ABT-737: An ex vivo experiment in human serous ovarian carcinoma. Int $\mathrm{J}$ Cancer. 2015; 136(5):E340-350.

578. Meynet O, Zunino B, Happo L, Pradelli LA, Chiche J, Jacquin MA, Mondragon L, Tanti JF, Taillan B, Garnier G, Reverso-Meinietti J, Mounier N, Michiels JF, Michalak EM, Carles M, Scott CL, et al. Caloric restriction modulates Mcl-1 expression and sensitizes lymphomas to $\mathrm{BH} 3$ mimetic in mice. Blood. 2013; 122(14):2402-2411.

579. He L, Torres-Lockhart K, Forster N, Ramakrishnan S, Greninger P, Garnett MJ, McDermott U, Rothenberg SM, Benes $\mathrm{CH}$ and Ellisen LW. Mcl-1 and FBW7 control a dominant survival pathway underlying HDAC and Bcl2 inhibitor synergy in squamous cell carcinoma. Cancer Discov. 2013; 3(3):324-337.

580. Wilson WH, O'Connor OA, Czuczman MS, LaCasce AS, Gerecitano JF, Leonard JP, Tulpule A, Dunleavy K, Xiong H, Chiu YL, Cui Y, Busman T, Elmore SW, Rosenberg SH, Krivoshik AP, Enschede SH, et al. Navitoclax, a targeted high-affinity inhibitor of BCL-2, in lymphoid malignancies: a phase 1 dose-escalation study of safety, pharmacokinetics, pharmacodynamics, and antitumour activity. Lancet Oncol. 2010; 11(12):1149-1159.

581. Cleary JM, Lima CM, Hurwitz HI, Montero AJ, Franklin C, Yang J, Graham A, Busman T, Mabry M, Holen K, Shapiro GI and Uronis H. A phase I clinical trial of navitoclax, a targeted high-affinity Bcl-2 family inhibitor, in combination with gemcitabine in patients with solid tumors. Invest New Drugs. 2014; 32(5):937-945.

582. Kipps TJ, Eradat H, Grosicki S, Catalano J, Cosolo W, Dyagil I, Yalamanchili S, Chai A, Saharanaman S, Punnoose E, Hurst D and Pylypenko H. A phase 2 study of the BH3 mimetic BCL2 inhibitor navitoclax (ABT-263) with or without rituximab, in previously untreated B-cell chronic lymphocytic leukemia. Leuk Lymphoma. 2015:130. 
583. Tse C, Shoemaker AR, Adickes J, Anderson MG, Chen J, Jin S, Johnson EF, Marsh KC, Mitten MJ, Nimmer P, Roberts L, Tahir SK, Xiao Y, Yang X, Zhang H, Fesik S, et al. ABT-263: a potent and orally bioavailable Bcl-2 family inhibitor. Cancer Res. 2008; 68(9):3421-3428.

584. Faber AC, Farago AF, Costa C, Dastur A, Gomez-Caraballo M, Robbins R, Wagner BL, Rideout WM, 3rd, Jakubik CT, Ham J, Edelman EJ, Ebi H, Yeo AT, Hata AN, Song Y, Patel NU, et al. Assessment of ABT-263 activity across a cancer cell line collection leads to a potent combination therapy for small-cell lung cancer. Proc Natl Acad Sci U S A. $2015 ; 112(11):$ E1288-1296.

585. Alford SE, Kothari A, Loeff FC, Eichhorn JM, Sakurikar N, Goselink HM, Saylors RL, Jedema I, Falkenburg JH and Chambers TC. BH3 Inhibitor Sensitivity and Bcl-2 Dependence in Primary Acute Lymphoblastic Leukemia Cells. Cancer Res. 2015; 75(7):1366-1375.

586. Choudhary GS, Al-Harbi S, Mazumder S, Hill BT, Smith MR, Bodo J, Hsi ED and Almasan A. MCL-1 and BCL-xLdependent resistance to the BCL-2 inhibitor ABT-199 can be overcome by preventing PI3K/AKT/mTOR activation in lymphoid malignancies. Cell Death Dis. 2015; 6:e1593.

587. Nguyen M, Marcellus RC, Roulston A, Watson M, Serfass L, Murthy Madiraju SR, Goulet D, Viallet J, Belec L, Billot X, Acoca S, Purisima E, Wiegmans A, Cluse L, Johnstone RW, Beauparlant P, et al. Small molecule obatoclax (GX15070) antagonizes MCL-1 and overcomes MCL-1-mediated resistance to apoptosis. Proc Natl Acad Sci U S A. 2007; 104(49):19512-19517.

588. Vogler M, Weber K, Dinsdale D, Schmitz I, SchulzeOsthoff K, Dyer MJ and Cohen GM. Different forms of cell death induced by putative BCL2 inhibitors. Cell Death Differ. 2009; 16(7):1030-1039.

589. Albershardt TC, Salerni BL, Soderquist RS, Bates DJ, Pletnev AA, Kisselev AF and Eastman A. Multiple BH3 mimetics antagonize antiapoptotic MCL1 protein by inducing the endoplasmic reticulum stress response and upregulating BH3-only protein NOXA. J Biol Chem. 2011; 286(28):24882-24895.

590. Urtishak KA, Edwards AY, Wang LS, Hudome A, Robinson BW, Barrett JS, Cao K, Cory L, Moore JS, Bantly AD, Yu QC, Chen IM, Atlas SR, Willman CL, Kundu M, Carroll AJ, et al. Potent obatoclax cytotoxicity and activation of triple death mode killing across infant acute lymphoblastic leukemia. Blood. 2013; 121(14):2689-2703.

591. Trudel S, Li ZH, Rauw J, Tiedemann RE, Wen XY and Stewart AK. Preclinical studies of the pan-Bcl inhibitor obatoclax (GX015-070) in multiple myeloma. Blood. 2007; 109(12):5430-5438.

592. Spijkers-Hagelstein JA, Schneider P, Pinhancos SM, Garrido Castro P, Pieters R and Stam RW. Glucocorticoid sensitisation in Mixed Lineage Leukaemia-rearranged acute lymphoblastic leukaemia by the pan-BCL-2 family inhibitors gossypol and AT-101. Eur J Cancer. 2014; 50(9):1665-1674.
593. Leverson JD, Zhang H, Chen J, Tahir SK, Phillips DC, Xue J, Nimmer P, Jin S, Smith M, Xiao Y, Kovar P, Tanaka A, Bruncko M, Sheppard GS, Wang L, Gierke S, et al. Potent and selective small-molecule MCL-1 inhibitors demonstrate on-target cancer cell killing activity as single agents and in combination with ABT-263 (navitoclax). Cell Death Dis. 2015; 6:e1590.

594. Pan R, Ruvolo VR, Wei J, Konopleva M, Reed JC, Pellecchia M, Andreeff M and Ruvolo PP. Inhibition of Mcl-1 with the pan-Bcl-2 family inhibitor (-)BI97D6 overcomes ABT-737 resistance in acute myeloid leukemia. Blood. 2015; 126(3):363-372.

595. Kazi A, Sun J, Doi K, Sung SS, Takahashi Y, Yin H, Rodriguez JM, Becerril J, Berndt N, Hamilton AD, Wang HG and Sebti SM. The BH3 alpha-helical mimic BH3-M6 disrupts $\mathrm{Bcl}-\mathrm{X}(\mathrm{L}), \mathrm{Bcl}-2$, and MCL-1 protein-protein interactions with $\mathrm{Bax}, \mathrm{Bak}, \mathrm{Bad}$, or Bim and induces apoptosis in a Bax- and Bim-dependent manner. J Biol Chem. 2011; 286(11):9382-9392.

596. LaBelle JL, Katz SG, Bird GH, Gavathiotis E, Stewart ML, Lawrence C, Fisher JK, Godes M, Pitter K, Kung AL and Walensky LD. A stapled BIM peptide overcomes apoptotic resistance in hematologic cancers. J Clin Invest. 2012; 122(6):2018-2031.

597. Guo X, Zhou CY, Li Q, Gao J, Zhu YP, Gu L and Ma ZG. Rapamycin sensitizes glucocorticoid resistant acute lymphoblastic leukemia CEM-C1 cells to dexamethasone induced apoptosis through both mTOR suppression and up-regulation and activation of glucocorticoid receptor. Biomed Environ Sci. 2013; 26(5):371-381.

598. Gu L, Gao J, Li Q, Zhu YP, Jia CS, Fu RY, Chen Y, Liao QK and Ma Z. Rapamycin reverses NPM-ALK-induced glucocorticoid resistance in lymphoid tumor cells by inhibiting mTOR signaling pathway, enhancing G1 cell cycle arrest and apoptosis. Leukemia. 2008; 22(11):20912096.

599. Zhang C, Ryu YK, Chen TZ, Hall CP, Webster DR and Kang MH. Synergistic activity of rapamycin and dexamethasone in vitro and in vivo in acute lymphoblastic leukemia via cell-cycle arrest and apoptosis. Leuk Res. 2012; 36(3):342-349.

600. Xu ZZ, Wang WF, Fu WB, Wang AH, Liu ZY, Chen LY, Guo $\mathrm{P}$ and Li JM. Combination of rituximab and mammalian target of rapamycin inhibitor everolimus (RAD001) in diffuse large B-cell lymphoma. Leuk Lymphoma. 2014; 55(5):1151-1157.

601. Gomez-Benito M, Balsas P, Carvajal-Vergara X, Pandiella A, Anel A, Marzo I and Naval J. Mechanism of apoptosis induced by IFN-alpha in human myeloma cells: role of Jak1 and Bim and potentiation by rapamycin. Cell Signal. 2007; 19(4):844-854.

602. Fang L, Wang H, Zhou L and Yu D. FOXO3a reactivation mediates the synergistic cytotoxic effects of rapamycin and cisplatin in oral squamous cell carcinoma cells. Toxicol Appl Pharmacol. 2011; 251(1):8-15. 
603. Wu L, Birle DC and Tannock IF. Effects of the mammalian target of rapamycin inhibitor CCI-779 used alone or with chemotherapy on human prostate cancer cells and xenografts. Cancer Res. 2005; 65(7):2825-2831.

604. Mondesire WH, Jian W, Zhang H, Ensor J, Hung MC, Mills GB and Meric-Bernstam F. Targeting mammalian target of rapamycin synergistically enhances chemotherapy-induced cytotoxicity in breast cancer cells. Clin Cancer Res. 2004; 10(20):7031-7042.

605. Coloff JL, Macintyre AN, Nichols AG, Liu T, Gallo CA, Plas DR and Rathmell JC. Akt-dependent glucose metabolism promotes Mcl-1 synthesis to maintain cell survival and resistance to Bcl-2 inhibition. Cancer Res. 2011; 71(15):5204-5213.

606. Garber K. Targeting mTOR: something old, something new. J Natl Cancer Inst. 2009; 101(5):288-290.

607. Li Z, Yan S, Attayan N, Ramalingam S and Thiele CJ. Combination of an allosteric Akt Inhibitor MK-2206 with etoposide or rapamycin enhances the antitumor growth effect in neuroblastoma. Clin Cancer Res. 2012; 18(13):3603-3615.

608. Faber AC, Coffee EM, Costa C, Dastur A, Ebi H, Hata AN, Yeo AT, Edelman EJ, Song Y, Tam AT, Boisvert JL, Milano RJ, Roper J, Kodack DP, Jain RK, Corcoran RB, et al. mTOR inhibition specifically sensitizes colorectal cancers with KRAS or BRAF mutations to BCL-2/BCLXL inhibition by suppressing MCL-1. Cancer Discov. 2014; 4(1):42-52.

609. Zhao L, Teng B, Wen L, Feng Q, Wang H, Li N, Wang $\mathrm{Y}$ and Liang Z. mTOR inhibitor AZD8055 inhibits proliferation and induces apoptosis in laryngeal carcinoma. Int J Clin Exp Med. 2014; 7(2):337-347.

610. Gupta M, Hendrickson AE, Yun SS, Han JJ, Schneider PA, Koh BD, Stenson MJ, Wellik LE, Shing JC, Peterson KL, Flatten KS, Hess AD, Smith BD, Karp JE, Barr S, Witzig TE, et al. Dual mTORC1/mTORC2 inhibition diminishes Akt activation and induces Puma-dependent apoptosis in lymphoid malignancies. Blood. 2012; 119(2):476-487.

611. Rahmani M, Aust MM, Attkisson E, Williams DC, Jr., Ferreira-Gonzalez A and Grant S. Inhibition of Bcl-2 antiapoptotic members by obatoclax potently enhances sorafenib-induced apoptosis in human myeloid leukemia cells through a Bim-dependent process. Blood. 2012; 119(25):6089-6098.

612. Zhang W, Konopleva M, Ruvolo VR, McQueen T, Evans RL, Bornmann WG, McCubrey J, Cortes J and Andreeff M. Sorafenib induces apoptosis of AML cells via Bimmediated activation of the intrinsic apoptotic pathway. Leukemia. 2008; 22(4):808-818.

613. Chen S, Dai Y, Pei XY, Myers J, Wang L, Kramer LB, Garnett M, Schwartz DM, Su F, Simmons GL, Richey JD, Larsen DG, Dent P, Orlowski RZ and Grant S. CDK inhibitors upregulate $\mathrm{BH} 3$-only proteins to sensitize human myeloma cells to $\mathrm{BH} 3$ mimetic therapies. Cancer Res. 2012; 72(16):4225-4237.
614. Baumann P, Schneider L, Mandl-Weber S, Oduncu F and Schmidmaier R. Simultaneous targeting of PI3K and mTOR with NVP-BGT226 is highly effective in multiple myeloma. Anticancer Drugs. 2012; 23(1):131-138.

615. Graf N, Li Z, Herrmann K, Weh D, Aichler M, Slawska J, Walch A, Peschel C, Schwaiger M, Buck AK, Dechow T and Keller U. Positron emission tomographic monitoring of dual phosphatidylinositol-3-kinase and mTOR inhibition in anaplastic large cell lymphoma. Onco Targets Ther. 2014; 7:789-798.

616. Simioni C, Cani A, Martelli AM, Zauli G, Alameen AA, Ultimo S, Tabellini G, McCubrey JA, Capitani S and Neri LM. The novel dual PI3K/mTOR inhibitor NVP-BGT226 displays cytotoxic activity in both normoxic and hypoxic hepatocarcinoma cells. Oncotarget. 2015; 6(19):1714717160.

617. Bhat M, Robichaud N, Hulea L, Sonenberg N, Pelletier J and Topisirovic I. Targeting the translation machinery in cancer. Nat Rev Drug Discov. 2015; 14(4):261-278.

618. Faber AC, Corcoran RB, Ebi H, Sequist LV, Waltman BA, Chung E, Incio J, Digumarthy SR, Pollack SF, Song Y, Muzikansky A, Lifshits E, Roberge S, Coffman EJ, Benes $\mathrm{CH}$, Gomez HL, et al. BIM expression in treatment-naive cancers predicts responsiveness to kinase inhibitors. Cancer Discov. 2011; 1(4):352-365.

619. Wu DW, Chen CY, Chu CL and Lee H. Paxillin confers resistance to tyrosine kinase inhibitors in EGFR-mutant lung cancers via modulating BIM and Mcl-1 protein stability. Oncogene. 2015 Apr 27. doi: 10.1038/onc.2015.120. [Epub ahead of print].

620. Krysan K, Cui X, Gardner BK, Reckamp KL, Wang X, Hong L, Walser TC, Rodriguez NL, Pagano PC, Garon EB, Brothers JF, 2nd, Elashoff D, Lee JM, Spira AE, Sharma $\mathrm{S}$, Fishbein $\mathrm{MC}$, et al. Elevated neutrophil gelatinaseassociated lipocalin contributes to erlotinib resistance in non-small cell lung cancer. Am J Transl Res. 2013; 5(5):481-496.

621. Phuchareon J, McCormick F, Eisele DW and Tetsu O. EGFR inhibition evokes innate drug resistance in lung cancer cells by preventing Akt activity and thus inactivating Ets-1 function. Proc Natl Acad Sci U S A. 2015; 112(29):E3855-3863.

622. Watanuki Z, Kosai H, Osanai N, Ogama N, Mochizuki M, Tamai K, Yamaguchi K, Satoh K, Fukuhara T, Maemondo M, Ichinose M, Nukiwa T and Tanaka N. Synergistic cytotoxicity of afatinib and cetuximab against EGFR T790M involves Rab11-dependent EGFR recycling. Biochem Biophys Res Commun. 2014; 455(3-4):269-276.

623. Meng L, Shu M, Chen Y, Yang D, He Q, Zhao H, Feng Z, Liang $\mathrm{C}$ and $\mathrm{Yu} \mathrm{K}$. A novel lead compound CM-118: antitumor activity and new insight into the molecular mechanism and combination therapy strategy in c-Metand ALK-dependent cancers. Cancer Biol Ther. 2014; 15(6):721-734.

624. Shaw AT, Yasothan U and Kirkpatrick P. Crizotinib. Nat 
Rev Drug Discov. 2011; 10(12):897-898.

625. Tanizaki J, Okamoto I, Okamoto K, Takezawa K, Kuwata $\mathrm{K}$, Yamaguchi $\mathrm{H}$ and Nakagawa K. MET tyrosine kinase inhibitor crizotinib (PF-02341066) shows differential antitumor effects in non-small cell lung cancer according to MET alterations. J Thorac Oncol. 2011; 6(10):1624-1631.

626. Takezawa K, Okamoto I, Nishio K, Janne PA and Nakagawa K. Role of ERK-BIM and STAT3-survivin signaling pathways in ALK inhibitor-induced apoptosis in EML4-ALK-positive lung cancer. Clin Cancer Res. 2011; 17(8):2140-2148.

627. Okamoto W, Okamoto I, Arao T, Kuwata K, Hatashita E, Yamaguchi H, Sakai K, Yanagihara K, Nishio K and Nakagawa K. Antitumor action of the MET tyrosine kinase inhibitor crizotinib (PF-02341066) in gastric cancer positive for MET amplification. Mol Cancer Ther. 2012; 11(7):1557-1564.

628. Huynh H, Ong R and Soo KC. Foretinib demonstrates antitumor activity and improves overall survival in preclinical models of hepatocellular carcinoma. Angiogenesis. 2012; 15(1):59-70.

629. Dufies M, Jacquel A, Belhacene N, Robert G, Cluzeau T, Luciano F, Cassuto JP, Raynaud S and Auberger P. Mechanisms of AXL overexpression and function in Imatinib-resistant chronic myeloid leukemia cells. Oncotarget. 2011; 2(11):874-885.

630. Sinha S, Boysen J, Nelson M, Secreto C, Warner SL, Bearss DJ, Lesnick C, Shanafelt TD, Kay NE and Ghosh AK. Targeted Axl Inhibition Primes Chronic Lymphocytic Leukemia B Cells to Apoptosis and Shows Synergistic/ Additive Effects in Combination with BTK Inhibitors. Clin Cancer Res. 2015; 21(9):2115-2126.

631. Fedorenko IV, Abel EV, Koomen JM, Fang B, Wood ER, Chen YA, Fisher KJ, Iyengar S, Dahlman KB, Wargo JA, Flaherty KT, Sosman JA, Sondak VK, Messina JL, Gibney GT and Smalley KS. Fibronectin induction abrogates the BRAF inhibitor response of BRAF V600E/PTEN-null melanoma cells. Oncogene. 2015 Jun 15. doi: 10.1038/ onc.2015.188. [Epub ahead of print].

632. Phadke MS, Sini P and Smalley KS. The Novel ATPCompetitive MEK/Aurora Kinase Inhibitor BI-847325 Overcomes Acquired BRAF Inhibitor Resistance through Suppression of Mcl-1 and MEK Expression. Mol Cancer Ther. 2015; 14(6):1354-1364.

633. Joshi M, Rice SJ, Liu X, Miller B and Belani CP. Trametinib with or without vemurafenib in BRAF mutated non-small cell lung cancer. PLoS One. 2015; 10(2):e0118210.

634. Paraiso KH, Haarberg HE, Wood E, Rebecca VW, Chen YA, Xiang Y, Ribas A, Lo RS, Weber JS, Sondak VK, John JK, Sarnaik AA, Koomen JM and Smalley KS. The HSP90 inhibitor XL888 overcomes BRAF inhibitor resistance mediated through diverse mechanisms. Clin Cancer Res. 2012; 18(9):2502-2514.

635. Acquaviva J, Smith DL, Jimenez JP, Zhang C, Sequeira
M, He S, Sang J, Bates RC and Proia DA. Overcoming acquired BRAF inhibitor resistance in melanoma via targeted inhibition of Hsp90 with ganetespib. Mol Cancer Ther. 2014; 13(2):353-363.

636. Bachmann PS, Gorman R, Mackenzie KL, Lutze-Mann $\mathrm{L}$ and Lock RB. Dexamethasone resistance in B-cell precursor childhood acute lymphoblastic leukemia occurs downstream of ligand-induced nuclear translocation of the glucocorticoid receptor. Blood. 2005; 105(6):2519-2526.

637. Baptista MJ, Muntanola A, Calpe E, Abrisqueta P, Salamero O, Fernandez E, Codony C, Gine E, Kalko SG, Crespo $\mathrm{M}$ and Bosch F. Differential gene expression profile associated to apoptosis induced by dexamethasone in CLL cells according to IGHV/ZAP-70 status. Clin Cancer Res. 2012; 18(21):5924-5933.

638. Cartron PF, Loussouarn D, Campone M, Martin SA and Vallette FM. Prognostic impact of the expression/ phosphorylation of the BH3-only proteins of the BCL-2 family in glioblastoma multiforme. Cell Death Dis. 2012; $3: e 421$.

639. Ying HQ, Chen J, He BS, Pan YQ, Wang F, Deng QW, Sun HL, Liu X and Wang SK. The effect of BIM deletion polymorphism on intrinsic resistance and clinical outcome of cancer patient with kinase inhibitor therapy. Sci Rep. 2015; 5:11348.

640. Bardwell PD, Gu J, McCarthy D, Wallace C, Bryant S, Goess C, Mathieu S, Grinnell C, Erickson J, Rosenberg SH, Schwartz AJ, Hugunin M, Tarcsa E, Elmore SW, McRae B, Murtaza A, et al. The Bcl-2 family antagonist ABT-737 significantly inhibits multiple animal models of autoimmunity. J Immunol. 2009; 182(12):7482-7489.

641. Ho LJ, Luo SF and Lai JH. Biological effects of interleukin-6: Clinical applications in autoimmune diseases and cancers. Biochem Pharmacol. 2015; 97(1):16-26.

642. Guggino G, Giardina AR, Raimondo S, Giardina G, Sireci G, Dieli F, Peralta M, Alessandro R, Triolo G and Ciccia F. Targeting IL-6 signalling in early rheumatoid arthritis is followed by Th1 and Th17 suppression and Th2 expansion. Clin Exp Rheumatol. 2014; 32(1):77-81.

643. Chi H. Regulation and function of mTOR signalling in $\mathrm{T}$ cell fate decisions. Nat Rev Immunol. 2012; 12(5):325-338.

644. Francisco LM, Sage PT and Sharpe AH. The PD-1 pathway in tolerance and autoimmunity. Immunol Rev. 2010; 236:219-242.

645. Kim HJ, Yoon KA, Yoon HJ, Hong JM, Lee MJ, Lee IK and Kim SY. Liver $\mathrm{X}$ receptor activation inhibits osteoclastogenesis by suppressing NF-kappaB activity and c-Fos induction and prevents inflammatory bone loss in mice. J Leukoc Biol. 2013; 94(1):99-107.

646. Kuribara R, Honda H, Matsui H, Shinjyo T, Inukai T, Sugita K, Nakazawa S, Hirai H, Ozawa K and Inaba T. Roles of Bim in apoptosis of normal and Bcr-Ablexpressing hematopoietic progenitors. Mol Cell Biol. 2004; 24(14):6172-6183. 
647. Santidrian AF, Gonzalez-Girones DM, Iglesias-Serret D, Coll-Mulet L, Cosialls AM, de Frias M, Campas C, Gonzalez-Barca E, Alonso E, Labi V, Viollet B, Benito A, Pons G, Villunger A and Gil J. AICAR induces apoptosis independently of AMPK and p53 through up-regulation of the BH3-only proteins BIM and NOXA in chronic lymphocytic leukemia cells. Blood. 2010; 116(16):30233032.

648. Mahajan IM, Chen MD, Muro I, Robertson JD, Wright CW and Bratton SB. BH3-Only Protein BIM Mediates Heat Shock-Induced Apoptosis. PLoS One. 2014; 9(1):e84388.

649. Thomas HE and Kay TW. Intracellular pathways of pancreatic beta-cell apoptosis in type 1 diabetes. Diabetes Metab Res Rev. 2011; 27(8):790-796.

650. Catalan E, Jaime-Sanchez P, Aguilo N, Simon MM, Froelich CJ and Pardo J. Mouse cytotoxic T cell-derived granzyme $\mathrm{B}$ activates the mitochondrial cell death pathway in a Bim dependent fashion. J Biol Chem. 2015; 290(11):6868-6877.

651. Kodama T, Takehara T, Hikita H, Shimizu S, Shigekawa M, Li W, Miyagi T, Hosui A, Tatsumi T, Ishida H, Kanto T, Hiramatsu N, Yin XM and Hayashi N. BH3-only activator proteins Bid and Bim are dispensable for Bak/ Bax-dependent thrombocyte apoptosis induced by Bcl$\mathrm{xL}$ deficiency: molecular requisites for the mitochondrial pathway to apoptosis in platelets. J Biol Chem. 2011; 286(16):13905-13913.

652. Nordigarden A, Kraft M, Eliasson P, Labi V, Lam EW, Villunger A and Jonsson JI. BH3-only protein Bim more critical than Puma in tyrosine kinase inhibitor-induced apoptosis of human leukemic cells and transduced hematopoietic progenitors carrying oncogenic FLT3. Blood. 2009; 113(10):2302-2311.

653. Melarangi T, Zhuang J, Lin K, Rockliffe N, Bosanquet AG, Oates M, Slupsky JR and Pettitt AR. Glucocorticoid resistance in chronic lymphocytic leukaemia is associated with a failure of upregulated $\mathrm{Bim} / \mathrm{Bcl}-2$ complexes to activate Bax and Bak. Cell Death Dis. 2012; 3:e372.

654. Ajabnoor GM, Crook T and Coley HM. Paclitaxel resistance is associated with switch from apoptotic to autophagic cell death in MCF-7 breast cancer cells. Cell Death Dis. 2012; 3:e260.

655. Li R, Moudgil T, Ross HJ and Hu HM. Apoptosis of nonsmall-cell lung cancer cell lines after paclitaxel treatment involves the BH3-only proapoptotic protein Bim. Cell Death Differ. 2005; 12(3):292-303.

656. Kiyota M, Kuroda J, Yamamoto-Sugitani M, Shimura Y, Nakayama R, Nagoshi H, Mizutani S, Chinen Y, Sasaki N, Sakamoto N, Kobayashi T, Matsumoto Y, Horiike S and Taniwaki M. FTY720 induces apoptosis of chronic myelogenous leukemia cells via dual activation of BIM and BID and overcomes various types of resistance to tyrosine kinase inhibitors. Apoptosis. 2013; 18(11):1437-1446. 